\title{
Analysis and Design of Low-Profile Reconfigurable Leaky- Wave Antennas Based on Substrate Integrated Waveguide for 5G Millimeter-Wave Applications
}

\author{
by
}

Nima Javanbakht

A Thesis submitted to the Faculty of Graduate and Postdoctoral Affairs in partial fulfillment of the requirements for the degree of

Doctorate

in

Electrical Engineering

Carleton University

Ottawa, Ontario

(C) 2021, Nima Javanbakht 


\section{Abstract}

5G millimeter-wave (mm-wave) applications have several challenges, such as detection accuracy, interference from other channels, high path loss, covering several users in a dense area, and vulnerability to the environmental conditions. Several antenna technologies were proposed in this dissertation to address some of the above-mentioned challenges in 5G applications with a focus on wireless networks and vehicle to everything (V2X) communications.

Leaky-wave antennas (LWAs) are suitable candidates for 5G mm-wave applications due to their beam-scanning capability, compactness, low cost, and ease of fabrication. Substrate integrated waveguide (SIW) and half-mode substrate integrated waveguide (HMSIW) are suitable candidates for realizing LWAs because of their low-profile and integration capability.

Several design approaches were introduced in this dissertation to improve the performance of SIW/HMSIW LWAs in 5G mm-wave applications. Some of the proposed antennas have a relatively wide beam-scanning range, suitable for radar systems and V2X

communications, while others have small beam-squint, suitable for point-to-point communications and seeker antennas.

Tapering the side aperture of an HMSIW and the feed transition of a SIW resulted in side-lobe level (SLL) reduction to enhance the detection accuracy and reduce sensitivity to interference. Applying the proposed methods reduced the SLL of an HMSIW LWA and a SIW LWA to $-11.2 \mathrm{~dB}$ and $-11.4 \mathrm{~dB}$, respectively, in the mm-wave frequency band. 
Furthermore, embedding cavities into a compact low temperature co-fired ceramics (LTCC) antenna enhanced the gain to $7.6 \mathrm{dBi}$ at $28.5 \mathrm{GHz}$.

Implementing different types of reconfigurable structures resulted in electronic beamscanning, which is the most suitable approach to provide coverage for several users in dense areas due to its ease of implementation. Each of the proposed reconfigurable antennas posed different scanning ranges. One example used varactor diodes for tuning the antenna and achieved $30^{\circ}$ of beam-scanning range by varying the varactor diode's capacitance in the range of 200-500fF. Moreover, the bias circuitry was integrated into the RF ground in a few designs to miniaturize the reconfigurable antenna. The compactness, beam-scanning capability, low SLL, medium to high gain, and low fabrication cost are among the features that make the proposed antennas suitable candidates for $5 \mathrm{G}$ mm-wave applications. 


\section{Acknowledgements}

I want to thank my wonderful family, Mr. Saeed Javanbakht, Mrs. Mozhdeh Etminan, and Ms. Ayda Javanbakht, for all their supports. I also want to thank my magnificent supervisors, Prof. Barry Syrett, Prof. Rony E. Amaya, and Prof. Jafar Shaker, for their supports and guidance during my Ph.D. studies, as they are like mentors and role models to me. I am grateful to the Ph.D. examination committee, Prof. Dereck McNamara, Prof. Aldo Petosa, Prof. Yahya Antar, and Prof. Hafez Roshdy for their valuable and helpful comments. Moreover, I am thankful to Prof. Shulabh Gupta, Prof. Steven McGarry, Prof. Calvin Plett, Prof. Niall Tait, and Prof. Langis Roy for the help in my research and teaching. I would also like to thank all the amazing staff members of the Department of Electronics, notably Blazenka Power, Nagui Mikhail, Scott Bruce, Stephen McLaurin, Valerie Daley, Lisa Chiarelli, and Anna Lee. Finally, I want to thank my wonderful friends, Behzad Yadegari, Mohammad Emara, Joseph Botros, William Che Knisely, Amin Esmailzadeh, Hooman Chamani, Amir Ali Derayatifar, Mohammad Torabzadeh, Armin Amyari, Pouria Tajvar, Shahrzad Khajavi, Aaron English, Joseph Hyland, Sonya Leonard, Joao Nizer, Ehsan Ghiasbegloo, Dan Gale, Brenden Korba, Paulyn Mulles, Alex Ross, Mehrnaz Moareb, Morvarid Basiri, Narges Ghiasi, Ariane Daigle, Reza Mottaghi, Benyamin Salehpour, Ville Tiukuvaara, Mohammad Farahani, Ahmed Aldehshan, Brittany Allison, Mars Nienhuis, Jorden Labossierre, Wenyu Zhou, Rene Nyangezi, and all my amazing friends all around the world who make my Ph.D. journey a joyful one. 


\section{Table of Contents}

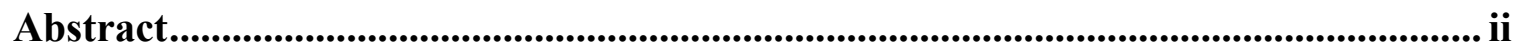

Acknowledgements ......................................................................................................................... iv

Table of Contents ....................................................................................................................... v

List of Tables .............................................................................................................................. viii

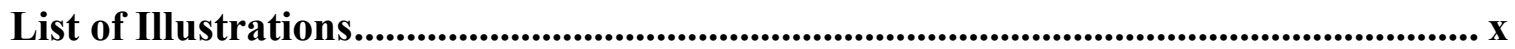

List of Abbreviations .......................................................................................................... Xxv

Chapter 1: Introduction ................................................................................................................... 1

$1.1 \quad$ Motivation and Objectives ………………………………………………….. 1

$1.2 \quad$ Methodology

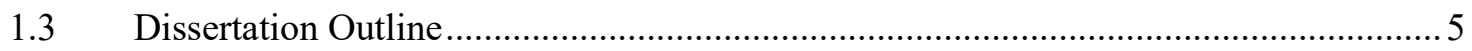

Chapter 2: Literature Review......................................................................................... 7

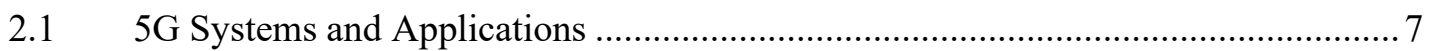

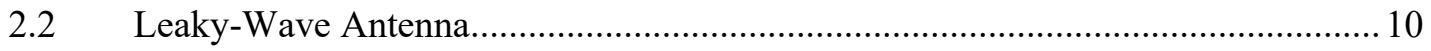

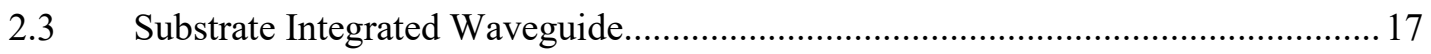

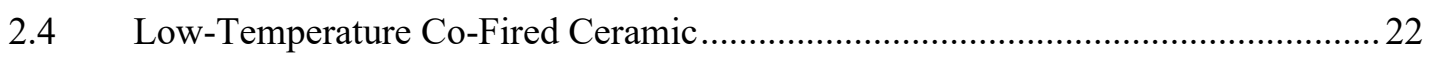

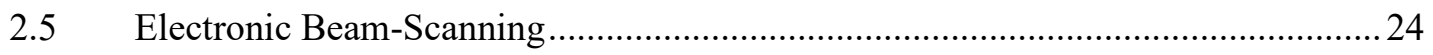

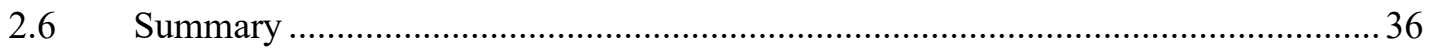

Chapter 3: Side-Lobe Level Reduction in HMSIW/SIW LWAs.................................... 38

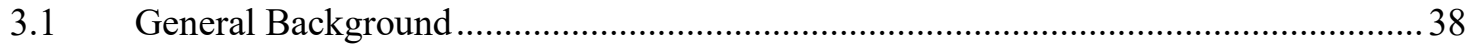

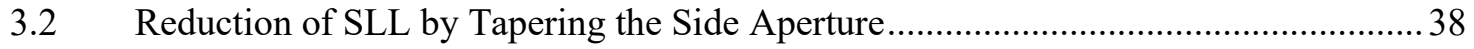

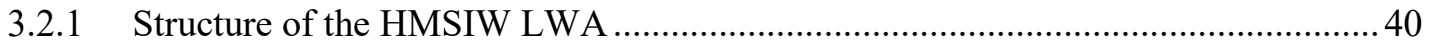

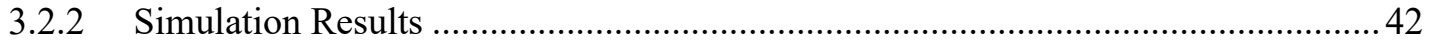

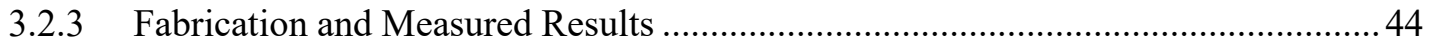




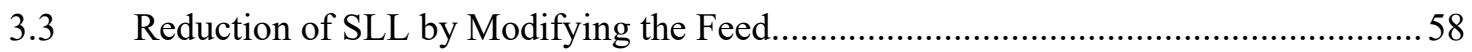

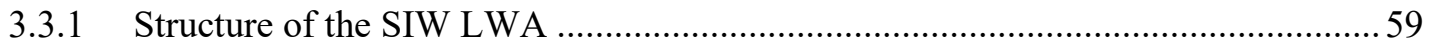

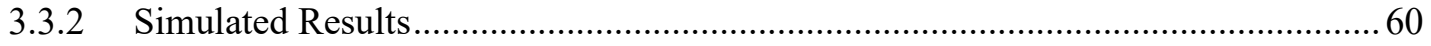

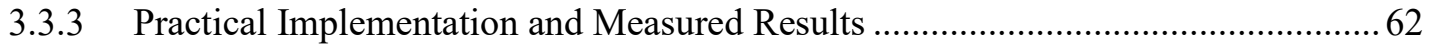

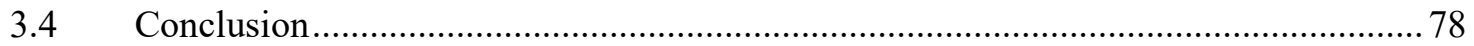

Chapter 4: Gain Enhancement of Compact HMSIW LWAs ...................................... 80

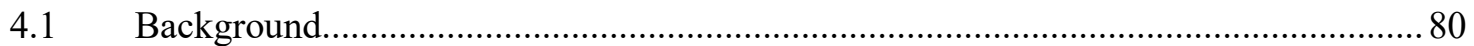

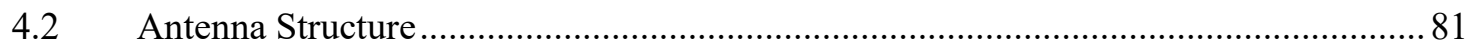

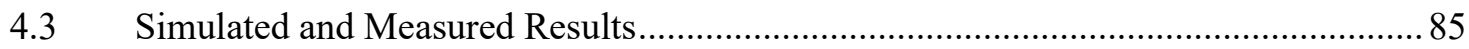

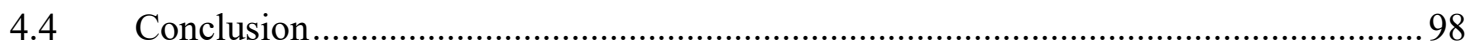

Chapter 5: Electronic Beam-Scanning in Low-Profile LWAs.................................... 101

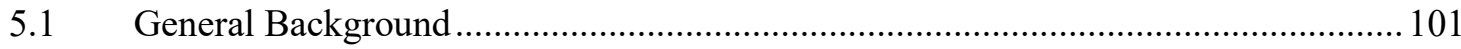

5.2 Reconfigurable Antennas Based on Periodic Cells with Negative Permittivity ......... 102

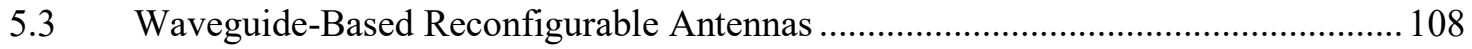

5.3.1 Reconfigurable HMSIW LWA with Patches ..................................................... 110

5.3.2 Reconfigurable HMSIW LWA with Circular Slots ............................................ 112

5.3.3 HMSIW LWA with Reconfigurable Cells in V-Shape Configuration on the

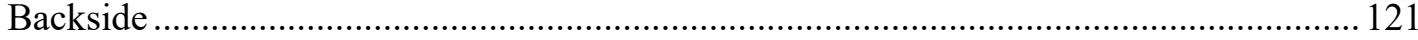

5.3.4 Reconfigurable SIW LWA with Transverse Slots .............................................. 140

5.3.5 Reconfigurable SIW LWA with Offset Longitudinal Slots ................................. 144

5.3.6 Reconfigurable SIW LWA Composed of Longitudinal Slots on the Backside ..... 147

5.3.7 Miniaturized SIW LWA with Offset Longitudinal Slot....................................... 158

5.3.8 Miniaturized Reconfigurable SIW LWA with Oblique Slots .............................. 162

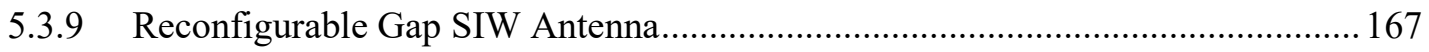

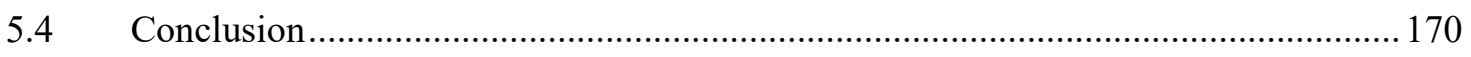


Chapter 6: Conclusion ................................................................................................................ 175

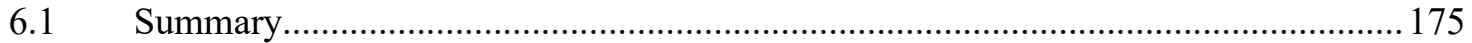

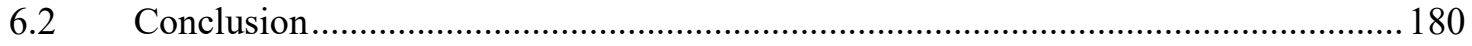

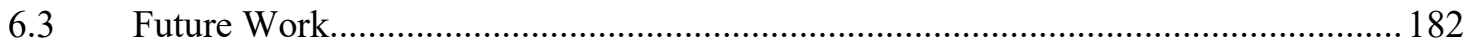

6.4 Contributions to Research Established by Published Papers...................................... 185

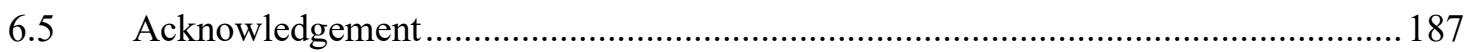

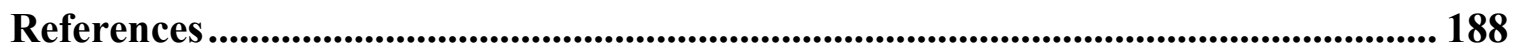




\section{List of Tables}

Table 3.1. Some of the important geometrical parameters of the proposed LWA [70]. .. 45

Table 3.2. Comparison among similar antennas in terms of SLL, peak gain, frequency

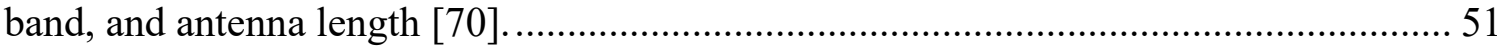

Table 3.3. The peak realized gain and SLL of the modified fed and conventional SIW

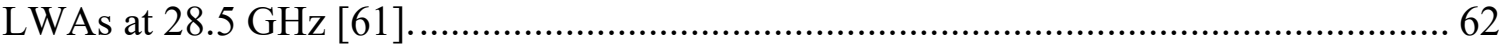

Table 3.4. A summary of the geometrical parameters of the antenna [62]...................... 63

Table 3.5. Comparison between different methods of SLL improvement [62]............... 77

Table 4.1. Some of the geometrical parameters of the proposed LTCC antenna [87]. .... 84

Table 4.2. Comparison of the proposed cavity-based LTCC LWA with similar designs

Table 4.3. Comparison of the proposed antennas in Chapters 3 and 4......................... 100

Table 5.1. S-parameters of the HMSIW LWA with patches for different switching states

Table 5.2. Important geometrical parameters of the proposed reconfigurable HMSIW-

based antenna [103].

125

Table 5.3. Comparison among the similar reported electronic beam-scanning antennas

Table 5.4. Important geometrical parameters of the proposed LWA [115].

Table 5.5. Comparison Among the Similar Reported Electronic Beam-Steering Antennas [115] 158 
Table 5.6. A comparison among proposed reconfigurable antennas in Chapter 5 in terms of scanning range, peak gain, the absolute value of SLL (ASLL), and antenna length. 173 


\section{List of Illustrations}

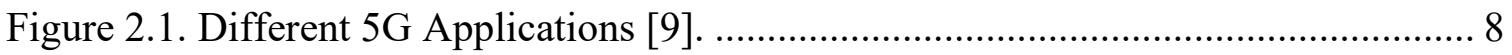

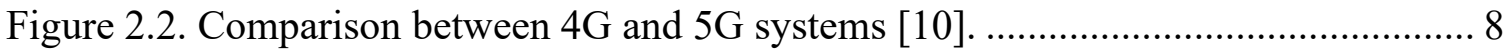

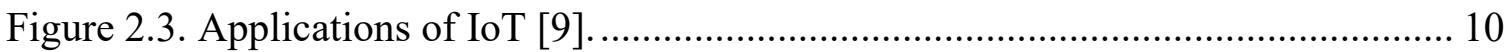

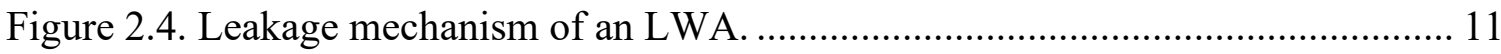

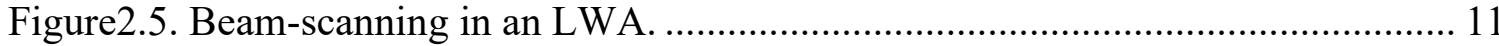

Figure 2.6. Dispersion diagram of a typical waveguide LWA....................................... 13

Figure 2.7. Schematic view of different types of an LWA. (a) ULWA, (b) QLWA, and (c)

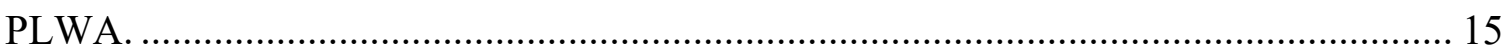

Figure 2.8. Proposed ULWA with reduced SLL in [48]............................................. 15

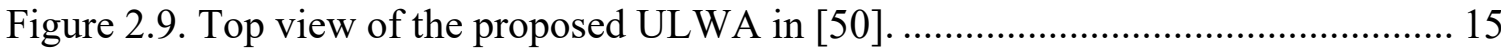

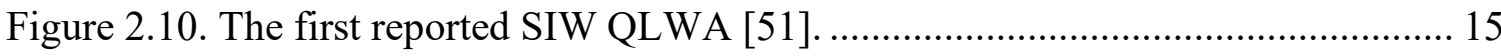

Figure 2.11. Tapered SIW QLWA with reduced SLL [52] ......................................... 16

Figure 2.12. Butterfly-shape QLWA with reduced SLL [54]......................................... 16

Figure 2.13. Thinned array QLWA based on air-filled waveguide [53]....................... 16

Figure 2.14. LWAs with additional via posts. (a) longitudinal slot [45], (b) transverse

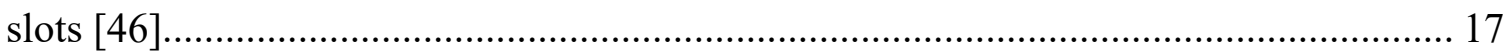

Figure 2.15. The first reported SIWs. (a) parallel plate waveguide with posts [42], (b)

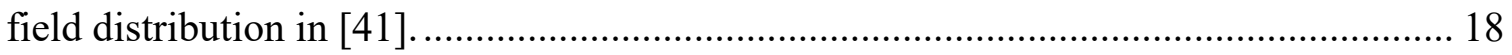

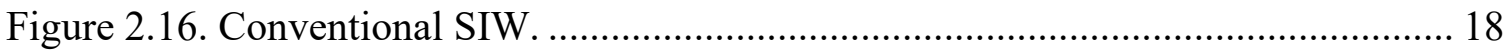

Figure 2.17. The first reported HMSIW. (a) expanded 3D view, (b) top view [63]........ 20 
Figure 2.18. Conventional HMSIW (a) top view, (b) electric field distribution of the dominant mode $\left(\mathrm{TE}_{0.5,0}\right)$ in HMSIW [76] ........................................................... 20

Figure 2.19. Proposed HMSIW LWA with tapered via fence for SLL reduction [65]. (a) the proposed method based on dividing the side via fence into multi-section [65], (b) schematic of the reported HMSIW antenna with reduced SLL [75] ........................... 21

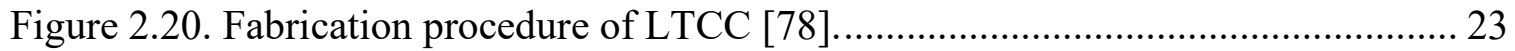
Figure 2.21. Vertical guiding structures based on LTCC [82]. (a) 3D view of the transition to SIW/horizontal waveguide, (b) 3D view of T-junction. 24 Figure 2.22. Different methods for achieving electronic beam-scanning [127]. (a) Using switches/varactor diodes, (b) Through MEMS, (c) Implementing liquid crystal. 25 Figure 2.23. Pixel antennas. (a) fabricated antenna with equal-sized patches [122], (b) fabricated antenna with different sizes patches [123], (c) radiation pattern at $2.5 \mathrm{GHz}$ [122], (d) measured radiation pattern at $2.45 \mathrm{GHz}$ [123]. 27

Figure 2.24. Equivalent circuit of CRLH. (a) conventional CRLH structure, (b) SIW-

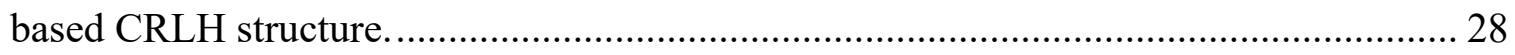
Figure 2.25. Dispersion diagram of a CRLH structure [149] .................................... 28 Figure 2.26. Reconfigurable CRLH antennas with shoring stubs and interdigital capacitors. (a) fabricated antenna proposed in [128], (b) fabricated structure designed in [129], (c) radiation pattern at $18 \mathrm{~V}$ [128], (d) radiation pattern at $2 \mathrm{~V}$ [128]. 29 Figure 2.27. Reconfigurable CRLH HMSIW LWA [99]. (a) schematic of the antenna, (b) radiation pattern at $6.5 \mathrm{GHz}$. 30 Figure 2.28. Reconfigurable HMSIW LWA with tunable grounded aperture [97] (a) schematic of the antenna, (b) radiation pattern at $6 \mathrm{GHz}$. 31 
Figure 2.29. Reconfigurable CRLH HMSIW LWA with tunable grounded aperture [104] (a) schematic of the antenna, (b) radiation pattern at $8.2 \mathrm{GHz}$ 31

Figure 2.30. Reconfigurable SIW LWA with variable via fences [108]. (a) schematic of the antenna, (b) radiation pattern at $5.2 \mathrm{GHz}$. 32 Figure 2.31. Reconfigurable corrugated SIW LWA with fan shape patches and open circuit stubs [109]. (a) schematic of the designed antenna, (b) radiation pattern at 5.8 $\mathrm{GHz}$

Figure 2.32. Reconfigurable cavity-backed SIW antenna with tunable posts [111]. (a) schematic of the antenna, (b) radiation pattern of the antenna at $2.6 \mathrm{GHz}$. 33

Figure 2.33. Schematic view of the proposed reconfigurable structure with negative $\varepsilon_{r}$ cells $[120]$ 34

Figure 2.34. Schematic view of the field-programmable microwave substrate (FPMS) [121]. (a) the proposed unit cell, (b) FPMS. 35

Figure 2.35. Reconfigurable waveguide realized on FPMS [121]. (a) $\mathrm{V}_{\text {bias }}=25 \mathrm{~V}$, (b) $\mathrm{V}_{\text {bias }}=10.72 \mathrm{~V}$

Figure 2.36. Schematic view of LTCC-based reconfigurable structure [121]. (a) master cell, (b) LTCC FPMS. 36

Figure 3.1. Electric field distributions of the conventional and tapered aperture HMSIW LWAs [70]. (a) Top view of E-field distribution in conventional HMSIW LWA (i.e., Wa $=0$ ), (b) Top view of E-field distribution in tapered aperture HMSIW LWA (i.e., Wa = $0.13 \mathrm{~mm}$ ), (c) 3D view of E-field distribution in conventional antenna, and (d) 3D view of E-field distribution in HMSIW LWA with tapered aperture. 
Figure 3.2. Schematic of the proposed HMSIW LWA with reduced SLL. (a) 3D view, (b)

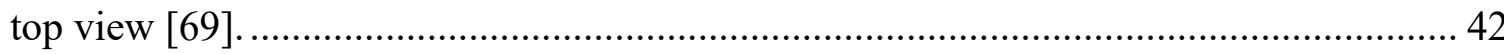

Figure 3.3. Different topologies for tapering the side aperture. (a) rectangular shape, (b) triangular/saw shape, (c) trapezoid shape. 43

Figure 3.4. Simulated S-parameters of the proposed 0.127mm HMSIW LWA with reduced SLL [69]. 43

Figure 3.5. Simulated radiation patterns of the proposed thin HMSIW LWA at E-plane (YZ-plane) $\left(\varphi=90^{\circ}\right)[69]$

Figure 3.6. Schematic view of the fabricated HMSIW LWA. (a) 3D view (tapered side aperture of the width of $W_{a}$ was circled in the same view), (b) the exaggerated non-scaled view of the tapered aperture, (c) top view, and (d) manufactured antenna [70]............. 46

Figure 3.7. S-parameters measurement setup of the proposed HMSIW LWA [70]........ 47

Figure 3.8. S-parameters of the fabricated HMSIW LWA with reduced SLL................ 47

Figure 3.9. Measurement setup for measuring the radiation pattern of the proposed HMSIW LWA. (a) antenna under test (AUT), (b) anechoic chamber [70] .................... 48 Figure 3.10. Radiation patterns of the fabricated HMSIW LWA at 28.5 GHz and E-plane $[70]$ 48

Figure 3.11. Radiation patterns of the proposed HMSIW LWA at E-plane $\left(\varphi=90^{\circ}\right)$ and different frequencies [70]. (a) at $28 \mathrm{GHz}$, (b) at $29 \mathrm{GHz}$, (c) $26.5 \mathrm{GHz}$, and (d) at 29.5 $\mathrm{GHz}$ 49

Figure 3.12. Peak realized gain of the proposed HMSIW LWA [70]. 51 
Figure 3.13. S-parameters of the proposed antenna for different combinations. (a) different values of $h$, (b) different values of $W$, (c) different values of $W_{a}$, and (d) different values of $W_{t}[70]$. 52

Figure 3.14. Normalized radiation patterns of the proposed HMSIW LWA obtained by changing different parameters at 28.5 GHz. (a) E-plane for different values of $h$ and $W_{a}$, (b) E-plane for different values of $W$ and $W_{t}$. [70]...... 53

Figure 3.15. Peak realized gain of the proposed HMSIW LWA obtained by changing different parameters [70]. 54

Figure 3.16. Schematic view of the proposed LWA with connectors offset from the horizon by $\xi[70]$. 54 Figure 3.17. S-parameters of the proposed HMSIW LWA with rotated connectors at 28.5 $\mathrm{GHz}[70]$. 55

Figure 3.18. Peak realized gain of the proposed HMSIW LWA obtained with offset connectors at $28.5 \mathrm{GHz}[70]$ 55

Figure 3.19. Normalized radiation patterns of the proposed HMSIW LWA with offset

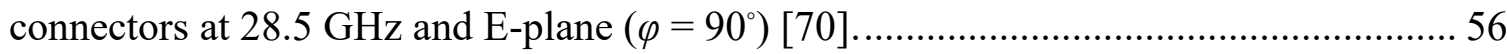

Figure 3.20. Schematic view of the bent antenna. (a) 3D view, (b) side view [70]........ 56 Figure 3.21. S-parameters of the proposed bent HMSIW LWA [70]............................ 57 Figure 3.22. Normalized radiation patterns of the proposed bent HMSIW LWA [70]... 57 Figure 3.23. Peak realized gain of the proposed bent HMSIW LWA [70]. 57 Figure 3.24. Schematic view of the proposed SLW LWA with reduced SLL. (a) 3D view, (b) top view [61]. 60 
Figure 3.25. S-parameters of the modified fed SIW LWA and the conventional one [61].

Figure 3.26. Normalized patterns of the conventional and the modified fed antennas at

$28.5 \mathrm{GHz}[61]$

61

Figure 3.27. The structure of the SIW LWA [62]. (a) 3D View, (b) top view of the LWA with the proposed feed structure, (c) top view of the conventional LWA (i.e., the antenna fed by microstrip line). 64

Figure 3.28. Measurement setup. (a) Antenna under test connected to VNA, (b) Antenna under test in the anechoic chamber [62]. 66

Figure 3.29. S-parameters of the fabricated SIW LWA with reduced SLL [62]. 66

Figure 3.30. Measured and simulated radiation patterns of the proposed antenna at 28.5 GHz and E-plane (YZ plane) [62]. 68

Figure 3.31. Measured and simulated radiation patterns of the proposed LWA at 28 and $29 \mathrm{GHz}[62]$. 68

Figure 3.32. S-parameters of the proposed SIW LWA with different parameters [62]. (a) $\mathrm{S}_{11}$ at different values of $h$ and $W$. (b) $\mathrm{S}_{21}$ at different values of $h$ and $W$. (c) $\mathrm{S}_{11}$ different values of $L_{s}, W_{s}, L_{m f}$, and $W_{m f .}$ (d) $\mathrm{S}_{21}$ different values of $L_{s}, W_{s}, L_{m f}$, and $W_{m f}$. 69 Figure 3.33. Normalized radiation patterns of the proposed antenna obtained by changing different parameters individually at $28.5 \mathrm{GHz}$ [62]. (a) E-plane for different values of $h$ and $W$, (b) E-plane for different values of $L_{s}$, (s) E-plane for different values of $W_{s}$, (d) Eplane for different values of $L_{m}$, and $W_{m f}$. 70 
Figure 3.34. Peak realized gain diagrams of the modified fed antenna corresponding to different parameters [62]. (a) different values of $h$ and $W$. (b) different values of $L_{s}, W_{s}$,

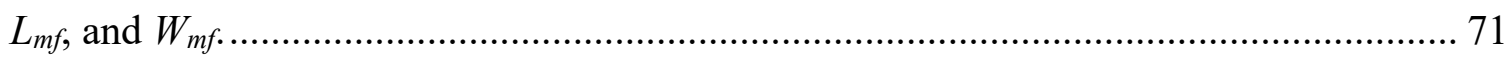

Figure 3.35. S-parameters of the proposed SIW LWA with different $W_{t}[62] \ldots \ldots \ldots \ldots \ldots . . .71$

Figure 3.36. Normalized radiation patterns of the SIW LWA with different $W_{t}$ at 28.5

$\mathrm{GHz}[62]$. 72

Figure 3.37. Peak realized gain diagrams of the modified fed antenna corresponding to different $W_{t}[62]$. 72

Figure 3.38. S-parameters of the proposed modified fed LWA with rotated connectors at $28.5 \mathrm{GHz}[62]$. 73

Figure 3.39. Normalized radiation patterns of the proposed SIW LWA with offset connectors at $28.5 \mathrm{GHz}$ and E-plane [62]. 73

Figure 3.40. Peak realized gain of the proposed modified fed LWA with offset connectors at $28.5 \mathrm{GHz}$ [62]. 74

Figure 3.41. Schematic view of the bent SIW LWA [62]. (a) 3D view, (b) side view. ... 74 Figure 3.42. S-parameters of the proposed bent LWA [62]. 75

Figure 3.43. Normalized radiation patterns of the proposed bent SIW LWA [62]. ........ 75

Figure 3.44. Peak realized gain of the proposed bent modified fed LWA [62]. 75

Figure 4.1. Schematic view of the proposed LTCC HMSIW LWA. (a) 3D view, (b) exaggerated view of the tapered aperture (non-scaled), (c) top view, (d) side view, and (e) fabricated antenna [87]. 83 Figure 4.2. Peak realized gain of the proposed LTCC LWA for different sizes of cavities $\left(L_{c}\right.$ and $\left.W_{c}\right)$ and their locations $\left(x_{c}\right.$ and $\left.z_{c}\right)$ at $28.5 \mathrm{GHz}$ [87]. 84 
Figure 4.3. Schematic view of the multi-layer conventional LTCC LWA and tapered height LTCC antenna [87]. (a) top view of the conventional non-loaded LTCC LWA, (b) top view of the tapered height LWA, (c) 3D view of the tapered height LWA, and (d) side view of the tapered height antenna. 85

Figure 4.4. Measured and simulated S-parameters of the proposed LTCC based antenna.

Figure 4.5. Measurement setup of the radiation pattern. (a) the AUT, (b) the anechoic chamber [87]. 86 Figure 4.6. Measured and simulated radiation patterns of the proposed LTCC LWA with embedded cavities and the multi-layer conventional non-loaded LTCC LWA at $28.5 \mathrm{GHz}$ and E-plane [87]. 88 Figure 4.7. Gain radiation patterns diagrams of the proposed LTCC LWA with embedded

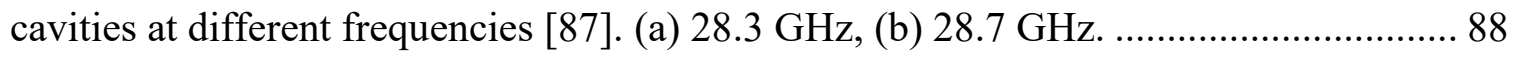
Figure 4.8. Peak realized gain diagrams of the proposed LTCC HMSIW LWA [87]. .... 90 Figure 4.9. Simulated peak realized gain of the tapered height LTCC LWA at $28.5 \mathrm{GHz}$ [87]. 90

Figure 4.10. S-parameters of the proposed LTCC LWA with different parameters [87]. (a) S11 for different values of h, d, and s, (b) S11 for different values of W, Lc, and Wc. (c) S21 for different values of h, d, and s, (d) S21 for different values of W, Lc, and Wc.

Figure 4.11. Normalized radiation patterns of the proposed LTCC antenna obtained from changing different parameters at $28.5 \mathrm{GHz}$ [87]. (a) different values of $h, d$, and $s$, (b) different values of $W, L_{c}$, and $W_{c}$. 92 
Figure 4.12. Peak realized gains of the LTCC antenna corresponding to different parameters [87]. (a) different values of $h, d$, and s, (b) different values of W, Lc, and Wc.

Figure 4.13. S-parameters of the proposed LTCC antenna with different Wt [87]......... 93

Figure 4.14. Normalized radiation patterns of the proposed LWA with different Wt at $28.5 \mathrm{GHz}[87]$. 93

Figure 4.15. Peak realized gain diagrams of the LTCC antenna correspond to different $W_{t}$ [87]. 94

Figure 4.16. S-parameters of the proposed LTCC LWA with offset connectors [87]. (a) at $28.5 \mathrm{GHz}$ versus $\xi$, (b) versus frequency. 95

Figure 4.17. Normalized radiation patterns of the proposed HMSIW LTCC LWA with connectors offset from the horizon at $28.5 \mathrm{GHz}$ [87].

Figure 4.18. Peak realized gain of the proposed LTCC antenna with rotated connectors [87]. (a) at $28.5 \mathrm{GHz}$ versus $\xi$, (b) versus frequency. 96 Figure 4.19. Schematic view of the bent LTCC antenna [87]. (a) 3D view, (b) side view. 96

Figure 4.20. S-parameters of the proposed bent LTCC LWA [87]. 97

Figure 4.21. Normalized radiation patterns of the proposed bent HMSIW LTCC LWA [87].

Figure 4.22. Peak realized gain of the proposed bent LTCC antenna [87] 97 Figure 5.1. LWA with negative $\varepsilon_{r}$ cells. (a) top view of the proposed antenna, (b) schematic view of the reconfigurable cell, and (c) electric field distribution for cells with $\varepsilon_{r}=-100$ 104 
Figure 5.2. Permittivity and permeability of the reconfigurable cells versus frequency. (a) $\varepsilon_{r}$ and $\mu_{r}$ for $\mathrm{V}_{\text {bias }}=-25 \mathrm{~V}$, (b) $\varepsilon_{r}$ and $\mu_{r}$ for $\mathrm{V}_{\text {bias }}=0 \mathrm{~V}$ 106

Figure 5.3. S-parameter diagrams of the proposed antenna with negative $\varepsilon_{r}$ cells at $\mathrm{V}_{\text {bias }}=$ $25 \mathrm{v}$. 107

Figure 5.4. The radiation pattern of the proposed antenna for different values of $\mathrm{W}_{2}$. (a) E-plane (YZ-plane), (b) XZ-plane. 107

Figure 5.5. Schematic view of the reconfigurable HMSIW LWA with patches. (a) 3D view, (b) top view [100]. 111

Figure 5.6. Radiation patterns of the reconfigurable HMSIW LWA with patches for different switching states and E-plane $\left(\varphi=90^{\circ}\right)[100]$ 112 Figure 5.7. Different topologies for the reconfigurable cells. (a) circular shape, (b) square shape, (c) triangular shape, and (d) hexangular shape. 113 Figure 5.8. Schematic view of the reconfigurable HMSIW LWA with circular slots [101]. (a) 3D view, (b) top view. 114 Figure 5.9. Schematic view of the reconfigurable HMSIW LWA with circular slots in Vshape topology [102]. (a) 3D view, (b) top view, and (c) side view. 115 Figure 5.10. S-parameters of the proposed reconfigurable HMSIW antenna for different longitudinal switching states. (a) $\mathrm{S}_{11}$, (b) $\mathrm{S}_{21}$. 117 Figure 5.11. Radiation patterns of reconfigurable HMSIW LWA with circular slot and different longitudinal switching states at 28.5 GHz and E-plane; (1) all on, (2) all off, (3) the first row (nearby the aperture) off, and (4) the last row (nearby the via fence) off. . 117 
Figure 5.12. S-parameters of the proposed HMSIW LWA for different transversal switching states [101]. (1) the first column (closest to the input) off, (2) the last column

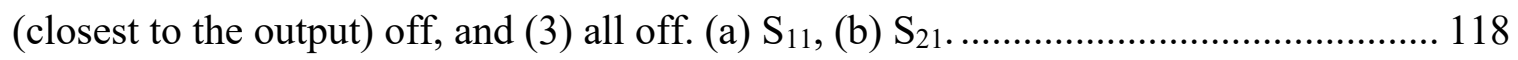
Figure 5.13. Radiation patterns of the reconfigurable HMSIW LWA with circular slot and transversal switching at $28.5 \mathrm{GHz}[101]$

Figure 5.14. S-parameters of the proposed reconfigurable HMSIW LWA with V-shape configuration [102]. (a) $\mathrm{S}_{11}$, (b) $\mathrm{S}_{21}$. 120

Figure 5.15. Radiation patterns of the reconfigurable HMSIW LWA with V-shape topology for different switching states at 28.5 GHz and E-plane (YZ plane); (1) all switches on, (2) all switches off, (3) the row close by the aperture (first row) off, and (4) the two nearest rows to the via fence (two last rows) off [102].... 120 Figure 5.16. The proposed reconfigurable HMSIW LWA composed of circular slots in a V-shape configuration [103]. (a) Bottom view, (b) Side view. 123 Figure 5.17. E-field distribution [103]. (a) Perspective view of HMSIW LWA without reconfigurable cells, (b) Top view of HMSIW LWA without reconfigurable cells....... 126 Figure 5.18. Measurement setup [103]. (a) S-parameters measurement setup, (b) Antenna under test in the anechoic chamber for measuring radiation pattern. Figure 5.19. S-parameters of the reconfigurable HMSIW LWA with reconfigurable cells in V-shape configuration [103]. (a) Return loss at states 1 and 2, (b) Return loss at states 3 and 4, (c) Insertion loss at states 1 and 2, and (d) Insertion loss at states 3 and 4..... 131 Figure 5.20. Gain radiation patterns of the proposed reconfigurable HMSIW LWA in Eplane (YZ-plane) for different switching states and frequencies [103]. (a) State 1 at 28 
GHz, (b) State 2 at $28 \mathrm{GHz}$, (c) State 3 at $28 \mathrm{GHz}$, (d) State 4 at $28 \mathrm{GHz}$, (e) State 1 at $28.5 \mathrm{GHz}$, (f) State 2 at $28.5 \mathrm{GHz}$

Figure 5.21. Peak realized gain of the proposed reconfigurable HMSIW LWA [103]. (a)

States 1 and 2, (b) States 3 and 4. 135

Figure 5.22. S-parameters for different geometrical parameters at state 2 [103]. (a) $\mathrm{S}_{11}$ for different values of $d, d_{2}, W_{p c}$, and $L_{p c}$, (b) $\mathrm{S}_{21}$ for different values of $d, d_{2}, W_{p c}$, and $L_{p c}$, (c) $\mathrm{S}_{11}$ for different values of $h$ and $W_{v}$, (d) $\mathrm{S}_{21}$ for different values of $h$ and $W_{v}$. 136

Figure 5.23. Radiation patterns at state 2 for different geometrical configurations [103].

(a) Different values of $h, d$, and $W_{v}$, (b) Different values of $d_{2}, W_{p c}$, and $L_{p c}$ 137

Figure 5.24. Schematic view of the bent reconfigurable HMSIW LWA [103]. (a) Side view, (b) 3D view. 137

Figure 5.25. S-parameters of the bent reconfigurable HMSIW LWA [103]. 137 Figure 5.26. Normalized radiation patterns of the bent reconfigurable HMSIW LWA at switching state 2 [103]. 138

Figure 5.27. Peak realized gain of the bent reconfigurable HMSIW LWA at switching state $2[103]$ 138

Figure 5.28. Schematic view of the reconfigurable SIW LWA with transverse slots [112]. (a) 3D view, (b) top view. 141

Figure 5.29. S-parameters of the proposed SIW LWA with uniform switching at 28.5

$\mathrm{GHz}[112]$. (a) $\mathrm{S}_{11}$, (b) $\mathrm{S}_{21}$ 142

Figure 5.30. Radiation patterns of the proposed reconfigurable SIW LWA with uniform switching at E-plane (YZ-plane) [112]. 143 
Figure 5.31. S-parameters of the proposed reconfigurable SIW LWA with non-uniform switching for different states (1) all off, (2) first half on, and (3) all on [113]. (a) $\mathrm{S}_{11}$, (b)

$\mathrm{S}_{21}$ 144

Figure 5.32. Radiation patterns of the proposed reconfigurable SIW LWA at $28.5 \mathrm{GHz}$ and E-plane for different non-uniform switching states (1) all off, (2) first half on, and (3) all on [113]. 144

Figure 5.33. Schematic view of the proposed reconfigurable SIW LWA with longitudinal slots [114]. (a) 3D view, (b) top view. 145

Figure 5.34. S-parameters of the proposed reconfigurable SIW LWA with offset longitudinal slots [114]. (a) $\mathrm{S}_{11}$, (b) $\mathrm{S}_{21}$. 146

Figure 5.35. Radiation patterns of the proposed reconfigurable SIW LWA with longitudinal slots for different switch capacitance values at $27.8 \mathrm{GHz}$ and E-plane (YZplane) $[114]$ 147

Figure 5.36. Schematic view of the proposed reconfigurable SIW-based LWA [115]. (a) Top view, (b) Side view. 148

Figure 5.37. Antenna under test in (a) S-parameters measurement, and (b) anechoic chamber [115]. 151

Figure 5.38. S-parameters of the proposed reconfigurable SIW LWA [115]. (a) $\mathrm{S}_{11}$ at states 1 and 2, (b) $\mathrm{S}_{21}$ at states 3 and 4, (c) $\mathrm{S}_{11}$ at states 1 and 2, (d) $\mathrm{S}_{21}$ at states 3 and 4.

Figure 5.39. Normalized radiation patterns of the proposed electronic beam-steering antenna for different states in E-plane (YZ plane) [115]. (a) States 1 and 2 at 27GHz, (b) 
States 3 and 4 at $27 \mathrm{GHz}$, (c) States 1 and 2 at $28.5 \mathrm{GHz}$, (d) States 3 and 4 at $28.5 \mathrm{GHz}$, (e) States 1 and 2 at $30 \mathrm{GHz}$, (f) States 3 and 4 at $30 \mathrm{GHz}$. 153

Figure 5.40. Peak realized gain of the proposed reconfigurable antenna [115]. (a) States 1 and 2, (b) States 3 and 4 . 154

Figure 5.41. S-parameters for different geometrical parameters at state 2 [115]. (a) $\mathrm{S}_{11}$ for different values of $h, s, d$, and $W_{v}$, (b) $\mathrm{S}_{21}$ for different values of $h, s, d$, and $W_{v}$. 155

Figure 5.42. Radiation patterns for different geometrical configurations at state 2 and $28.5 \mathrm{GHz}$ [115]. (a) Different values of $s$ and $d$, (b) Different values of $h$ and $W_{v} \ldots \ldots . .156$ Figure 5.43. Bent reconfigurable antenna [115]. (a) 3D view, (b) Side view. ............... 156 Figure 5.44. S-parameters of the bent reconfigurable antenna [115] .......................... 156 Figure 5.45. Normalized radiation pattern of bent reconfigurable SIW LWA [115] .... 157 Figure 5.46. Peak realized gain of the bent electronic beam-scanning SIW LWA [115].

Figure 5.47. Schematic view of the proposed miniaturized reconfigurable antenna. (a) 3D view, (b) Top view, (c) Side view. 159

Figure 5.48. The simulated S-parameters of the proposed miniaturized reconfigurable LWA with longitudinal slots. (a) $\mathrm{S}_{11}$, (b) $\mathrm{S}_{21}$ 161

Figure 5.49. Radiation patterns of the proposed miniaturized reconfigurable SIW LWA composed of longitudinal slots. 162

Figure 5.50. Peak realized gain of the proposed miniaturized reconfigurable SIW LWA for different varactor capacitance values. 162 Figure 5.51. Schematic view of the proposed reconfigurable miniaturized LWA [116]. (a) 3D view, (b) Top view, (c) Bottom view. 164 
Figure 5.52. S Parameters of the proposed reconfigurable miniaturized LWA [116]. (a)

$\mathrm{S}_{11},(\mathrm{~b}) \mathrm{S}_{21}$ 165

Figure 5.53. Normalized radiation pattern of the proposed miniaturized LWA at $29 \mathrm{GHz}$ [116]. 166

Figure 5.54. Peak realized gain of the proposed reconfigurable miniaturized LWA [116]. 166 Figure 5.55. Schematic view of the proposed gap SIW LWA [117]. (a) 3D view, (b) top view 168 Figure 5.56. S Parameters of the proposed reconfigurable gap SIW LWA [117]. (a) $\mathrm{S}_{11}$, (b) $\mathrm{S}_{21}$ 169

Figure 5.57. Radiation Patterns of the proposed reconfigurable gap SIW LWA at 28.7 $\mathrm{GHz}$ for different switching capacitance values (1) 200fF, (2) 250fF, and (3) 500fF. (a) E-plane (YZ plane), (b) XY-plane [117]. 170 Figure 6.1. Different types of new reconfigurable cells currently under investigation. (a) linear array of reconfigurable cells with common ground, (b) planar array of reconfigurable cells with common ground, (c) LWA with reconfigurable cells at sides of transverse slots 183

Figure 6.2. Few arrays of HMSIW LWA with tapered side apertures. (a) Y-shape topology, (b) Star-shape topology 184 


\section{List of Abbreviations}

CPW

CRLH

FPMS

GA

GaAs

GaN

HMSIW

HPBW

IoT

LTCC

LWA

M2M

MEMS

MIMO

mm

NRW

OSB

PCB

PLWA

PSO

QLWA
Co-planar waveguide

Composite right-hand left-hand

Field programmable microwave substrate

Genetic algorithm

Gallium arsenide

Gallium nitride

Half-mode substrate integrated waveguide

Half-power beamwidth

Internet of things

Low temperature co-fired ceramic

Leaky-wave antenna

Machine to machine

Micro-electro mechanical systems

Multi-input multi-output

Millimeter

Nicolson Ross Weir

Open stop band

Printed circuit board

Periodic leaky-wave antenna

Particle swamp optimization

Quasi-uniform leaky-wave antenna 
SIW

SLL

ULWA

V2X
Substrate integrated waveguide

Sidelobe level

Uniform leaky-wave antenna

Vehicle to everything 


\section{Chapter 1: Introduction}

Antennas are among the critical parts of wireless communication networks. They must have low side-lobe level (SLL), low loss, medium to high gain, and often beam-scanning capability. The low SLL is required to boost the detection accuracy. Improving the gain is necessary to compensate for the path loss and the losses in the system. Beam-scanning capability is also needed for covering several users and detecting targets.

In this chapter, the motivations and objectives of this dissertation are discussed first. Then new methods for addressing some of the current challenges in 5G systems are introduced. A comprehensive literature review on $5 \mathrm{G}$ systems, leaky-wave antenna (LWA), substrate integrated waveguide (SIW), low temperature co-fired ceramic (LTCC), and electronic beam-scanning will be presented in Chapter 2.

\subsection{Motivation and Objectives}

The proposed antennas in this dissertation are designed for 5G millimeter-wave (mmwave) applications. A summary of 5G applications and challenges is presented first.

$5 \mathrm{G}$ is the latest established communications standard with several diverse applications including the internet of things (IoT) [1]-[2] and vehicle to everything (V2X) communications [1]-[2]. Research trends are pushing the use of higher frequency bands since the lower frequency bands tend to be very crowded [3]. Moreover, the shift to higher frequencies leads to compact communication devices. Extensive research has been carried out on the network topologies and suitable antennas for 5G systems [1]-[27]. Providing high data rates in dense environments, increasing bandwidth, covering a vast number of users, reducing the path loss, and reducing the power consumption are among the main objectives of $5 \mathrm{G}$ wireless systems [1], [9]. 
In wireless systems, antenna designers face several challenges, including antenna size, peak realized gain, and power consumption [2]. Reducing SLL is essential to enhance the detection accuracy and reduce interference from adjacent antennas and channels. Implementing beam-scanning antennas is necessary to cover a wide angular range simultaneously and provide communication links for several users in dense areas [11]. Vulnerability to the operational and environmental variations is another challenge in 5G networks that can be mitigated using an electronic beam-scanning antenna [9]. Development of an automated vehicle is a potential area for the application of reconfigurable antennas. Collision avoidance, adaptive cruise control, and V2X communications are among the main challenges in this application. Overall, implementing a low loss, compact, and beam-steerable antennas is crucial in 5G applications.

Implementing a phased array in a wireless network is costly and adds to the complexity of the system. Each array element needs to be connected to a transceiver individually, and setting up the array elements to steer simultaneously is a challenging task [2], [3]. This is where implementing LWAs [28]-[30] becomes useful. Furthermore, implementing an LWA instead of a phased array reduces the cost and complexity of 5G applications. LWAs are among medium to high gain beam-steerable antennas [28]-[34] and suitable candidates for $5 \mathrm{G}$ applications. An LWA can be realized using waveguide or microstrip structures [28]-[30]. The waveguide structure has lower loss but is bulkier compared to the microstrip structure. SIW [35]-[47] is a low-profile structure that mimics a rectangular waveguide using planar microstrip technology. Antenna designers lean toward the adoption of the SIW as the backbone of the LWAs [48]-[76]. SIW can be 
realized using printed circuit board (PCB) or LTCC [77]-[93] technologies. The packaging tolerance of LTCC is higher than PCB [77], while it is more expensive. Compactness, low cost, beam-scanning capability, and ease of fabrication are among the features that render a SIW LWA [48]-[76] a viable candidate for 5G applications such as IoT [2], V2X, and M2M communications [2]. As a result, SIW LWA was chosen as the structure of the proposed designs in this dissertation.

The proposed antennas in this dissertation were designed to satisfy different antenna specifications for $5 \mathrm{G}$ applications in terms of gain, SLL, and beam-scanning range [7], [18], [22]-[27]. Generally, 5G single-element antennas have gain of 8-15 dBi [22], while 5G arrays have gain of 21-28 $\mathrm{dBi}$ and SLL of $-18 \mathrm{~dB}$ [22]. According to 5G ETSI-EN 302217-4 RPE classes 1-4 standard, the required antennas' gain for 5G systems ranges from -30 to $23 \mathrm{dBi}$ depending on the main-lobe location in the frequency band of 24-30 $\mathrm{GHz}$ [23]. The antennas' desirable gain in 5G V2X is $3 \mathrm{dBi}$ [18], while the desirable SLL in $5 \mathrm{G} \mathrm{V} 2 \mathrm{X}$ system is about $-10 \mathrm{~dB}$ [27]. The required gains of 5G MIMO antennas are in the range of 3-10 $\mathrm{dBi}[22]$, whereas the SLL is about $-9 \mathrm{~dB}$ [7]. The desired gain, SLL, and beam-steering range of the antenna in $5 \mathrm{G}$ wireless communications are $10 \mathrm{dBi},-11$ $\mathrm{dB}$, and $50^{\circ}$, respectively [24]. The preferred gain, SLL, and beam-steering range of the antenna in $5 \mathrm{G}$ cellphones are $11 \mathrm{dBi},-11 \mathrm{~dB}$, and $60^{\circ}$, respectively [26].

\subsection{Methodology}

The focus of this dissertation is on developing new antenna technologies for $5 \mathrm{G} \mathrm{mm-}$ wave applications using a SIW/HMSIW-based LWA as the basic structure.

Different antennas are proposed to address the challenges in 5G applications, such as detection accuracy, interference, beam-scanning range, operational and environmental 
variations, path loss, and covering a vast number of users in dense areas. The proposed designs are suitable for different 5G applications, including blind-spot monitoring, radar, V2X communications, point-to-point communications, and wireless networks.

To enhance the detection accuracy, the SLL should be reduced. The proposed methods for reducing SLL are based on modifying the aperture of an HMSIW LWA and the feed transition of a SIW LWA. Aperture and feed modification of HMSIW and SIW LWAs reduced SLL to $-11.2 \mathrm{~dB}$ and $-11.4 \mathrm{~dB}$, respectively. The path loss can be compensated by increasing gain of the antenna, which is achieved by embedding cavities into a compact LTCC antenna. Doing so boosted the gain to $7.6 \mathrm{~dB}$ at $28.5 \mathrm{GHz}$.

Reconfigurable antennas offer electronic beam-scanning [94]-[133], a more common approach than frequency and mechanical beam-scanning because of its simplicity. Enhancement of the beam-scanning range can be achieved by implementing sets of compact reconfigurable cells. A GaAs varactor diode was chosen as the switch to achieve reconfigurability due to its advantages such as small footprint, high switching speed, and low loss. Sweeping the bias voltages of the embedded varactor diodes in different configurations led to the electronic beam-scanning in the range of $16^{\circ}$ to $50^{\circ}$.

Overall, the contributions of this Ph.D. dissertation can be listed as follows:

1. The SLL of HMSIW LWA is reduced by tapering the side aperture for the first time, which reduces the sensitivity to interference from other antennas.

2. The SLL of SIW LWA is improved by implementing a new tapered CPW as feed transition, which improves the detection accuracy.

3. The gain of a compact LWA is improved by implementing cavities in an LTCC structure for the first time. This partly compensates for the path loss in a wireless 
network. The high packaging tolerance of LTCC and compactness of the proposed antenna are among its additional features making it suitable for implementation in $5 \mathrm{G}$ wireless devices.

4. First of its kind, a programmable LWA based on new reconfigurable metamaterial cells is proposed. Sets of reconfigurable cells with negative permittivity act as the via fence and confine the field. Sweeping the bias voltage of the switches is similar to changing the width and cut-off frequency artificially. This leads to variations in propagation constant and electronic beam-scanning.

5. Implementing several reconfigurable cells in HMSIW LWAs leads to electronic beam-scanning with relatively small gain variations. Different topologies including circular reconfigurable cells in straight and V-shape configuration are designed to accommodate $5 \mathrm{G}$ beam-scanning applications.

6. Implementing several reconfigurable cells in transverse, longitudinal, and oblique configurations in SIW LWAs leads to electronic beam-scanning as well. Mounting electronic components on the backside of the antenna reduces the undesired blockage effect on the radiation pattern.

7. Embedding the bias circuitry into the ground plane results in the miniaturization of the electronic beam-scanning antennas for the first time.

\subsection{Dissertation Outline}

The organization of this dissertation is as follows. A comprehensive literature review is presented in Chapter 2. Two methods for reducing SLL are investigated in Chapter 3 to enhance the detection accuracy and robustness to interference in $5 \mathrm{G} \mathrm{mm}$-wave applications. A new approach for increasing the gain is introduced in Chapter 4 to 
compensate for $5 \mathrm{G}$ wireless network's path loss. Several reconfigurable LWAs are proposed in Chapter 5 to enable the required beam-scanning capability in $5 \mathrm{G}$ beamscanning applications. In Chapters 3, 4, and 5, the design procedure, antenna structure, simulations, and measurement results of the proposed antennas are discussed. Conclusions and a list of achievements are given in Chapter 6, along with possibilities for future work. 


\section{Chapter 2: Literature Review}

The proposed antennas in this dissertation are HMSIW- and SIW-based LWAs designed for 5G mm-wave applications. Moreover, LTCC technology was implemented to achieve a compact antenna with high packaging tolerance. To achieve a suitable antenna for beam-scanning applications, several electronic beam-scanning antennas were designed in this dissertation. In this chapter, a comprehensive literature review on $5 \mathrm{G}$ systems and applications, LWA, SIW, LTCC, and electronic beam-scanning is presented to become familiar with the corresponding state-of-the-art trends and challenges.

\subsection{G Systems and Applications}

New applications are being proposed and developed for the $5 \mathrm{G}$ communications standard [1]-[10], such as IoT [1], [2], V2X communications [1], [2], [14]-[18], machine to machine (M2M) communications, smart home, and remote medical service, as shown in Fig. 2.1 [9], [10]. 5G is expected to enable a revolutionary leap in terms of data rates, latency, connectivity, availability, reliability, and power efficiency [1].

$5 \mathrm{G}$ is built on the foundations of the current $4 \mathrm{G}$ systems. Both standards will co-exist for the foreseeable future. The main differences between $4 \mathrm{G}$ and $5 \mathrm{G}$ wireless systems are bandwidth, data rate, coverage range, and power consumption, as listed in Fig. 2.2 [10]. OFDM and OFDMA are the most suitable signal processing techniques for $5 \mathrm{G}$ wireless networks [3]. 


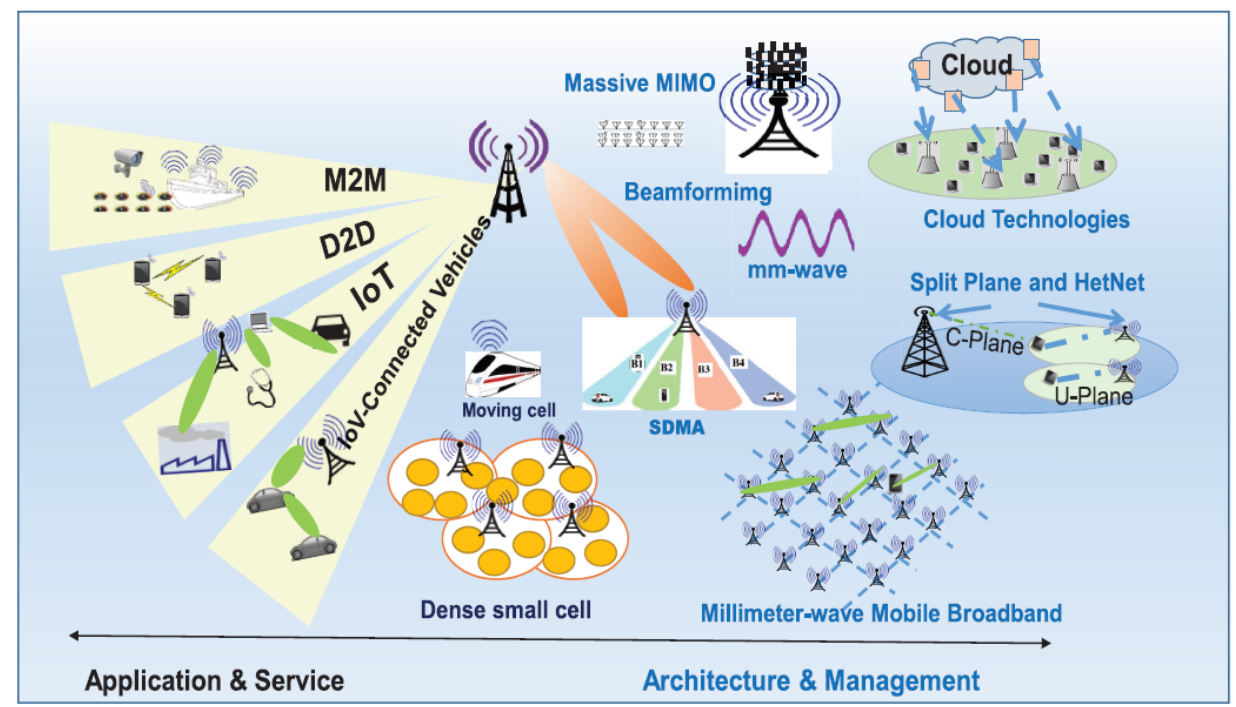

Figure 2.1. Different 5G Applications [9].

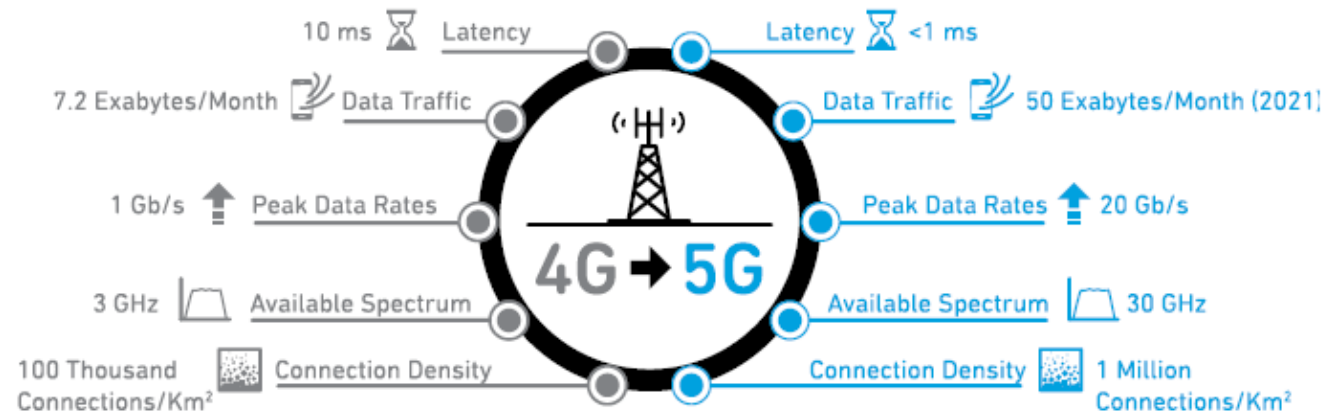

\begin{tabular}{|c|c|c|}
\hline & $\begin{array}{l}\text { 4G (Today, Before } \\
\text { Further Developments) }\end{array}$ & $5 G$ \\
\hline Latency & $10 \mathrm{~ms}$ & Less than $1 \mathrm{~ms}$ \\
\hline Peak data rates & 1 Gbps & 20 Gbps \\
\hline $\begin{array}{l}\text { Number of mobile } \\
\text { connections }\end{array}$ & 8 billion (2016) & 11 billion (2021) \\
\hline $\begin{array}{l}\text { Channel } \\
\text { bandwidth }\end{array}$ & $\begin{array}{l}20 \mathrm{MHz} \\
200 \mathrm{kHz} \text { (for Cat-NB1 loT) }\end{array}$ & $\begin{array}{l}\text { 100M } \mathrm{Mz} \text { below } 6 \mathrm{GHz} \\
400 \mathrm{MHz} \text { above } 6 \mathrm{GHz}\end{array}$ \\
\hline Frequency band & $600 \mathrm{MHz}$ to $5.925 \mathrm{GHz}$ & $\begin{array}{l}\text { 600MHz-mmWave (for } \\
\text { example, } 28 \mathrm{GHz}, 39 \mathrm{GHz} \text {, and } \\
\text { onward to } 80 \mathrm{GHz}\end{array}$ \\
\hline Uplink waveform & $\begin{array}{l}\text { Single-carrier frequency } \\
\text { division multiple } \\
\text { access (SC-FDMA) }\end{array}$ & $\begin{array}{l}\text { Option for cyclic prefix } \\
\text { orthogonal frequency-division } \\
\text { multiplexing (CP-OFDM) }\end{array}$ \\
\hline
\end{tabular}

Figure 2.2. Comparison between $4 \mathrm{G}$ and $5 \mathrm{G}$ systems [10].

The lower frequency bands are over-crowded by $4 \mathrm{G}$ and $3 \mathrm{G}$ systems. Therefore, research trends are pushing the use of higher frequency bands in 5G applications [3]. 
Moreover, operating in the higher frequencies leads to smaller devices, an essential advantage of operating at these frequency bands. There are several focused activities in the research community to enable relevant technologies for the allocated $5 \mathrm{G}$ mm-wave frequency bands [1], [2]. One of the suitable frequency bands for the mm-wave $5 \mathrm{G}$ systems is $24-32 \mathrm{GHz}$ due to the relatively small path loss [3] and low cost of the systems' elements. Generally, mm-wave frequency bands are good candidates for shortrange point to point communications since the loss is negligible in the short distance [3], [11]. However, mm-wave signals are more susceptible to interference from other antennas and adjacent channels [11]. Since the beams of the antennas are usually directive at the mm-wave band, the sensitivity to the misalignment is higher than the low frequency regime [3]. This is one of the key reasons that implementing beam-steerable antennas with low SLL is crucial in 5G mm-wave applications [12].

The promising features of $5 \mathrm{G}$ can be achieved by massive multi-input multi-output (MIMO) systems [2], [6], [7], [9], [10]. MIMO systems were introduced to 3G networks to improve network quality and enable access by several devices simultaneously. Equipping the base stations (BSs) with more antennas than the number of active users was the main idea behind the massive MIMO [3]. The size of the components in MIMO must be reduced as much as possible [10]. There are several methods for miniaturization, such as implementing LTCC-based devices. Implementing reconfigurable antennas in 5G massive MIMOs networks helps direct the antenna beam toward the user, improving the communication link quality [10].

IoT and V2X are among the most challenging applications of 5G. IoT allows several devices to simultaneously connect to the internet and communicate with each other 
wirelessly with extremely high efficiency and low cost [9], [10]. IoT make the realization of the smart cities, smart vehicles, and smart homes possible, as shown in Fig. 2.3. V2X provides high quality communication links between different moving vehicles and pedestrians [14]-[18]. Overall, implementing low power, low-profile, and beam-steerable antennas in V2X and IoT communications is crucial for keeping stable connections among moving users [14], [15].

\subsection{Leaky-Wave Antenna}

As stated before, realizing beam-steerable and medium to high gain antennas is crucial to overcome the path loss and vulnerability to the environment and operational variations [9]. LWA is a traveling wave antenna [28]-[31]. The radiation in an LWA occurs via the discontinuities on the guided structure, such as slots on the walls of a rectangular metallic waveguide. These antennas are called leaky-wave since the propagating wave gradually leaks into space through the discontinuities [28]-[31], as demonstrated in Fig. 2.4.

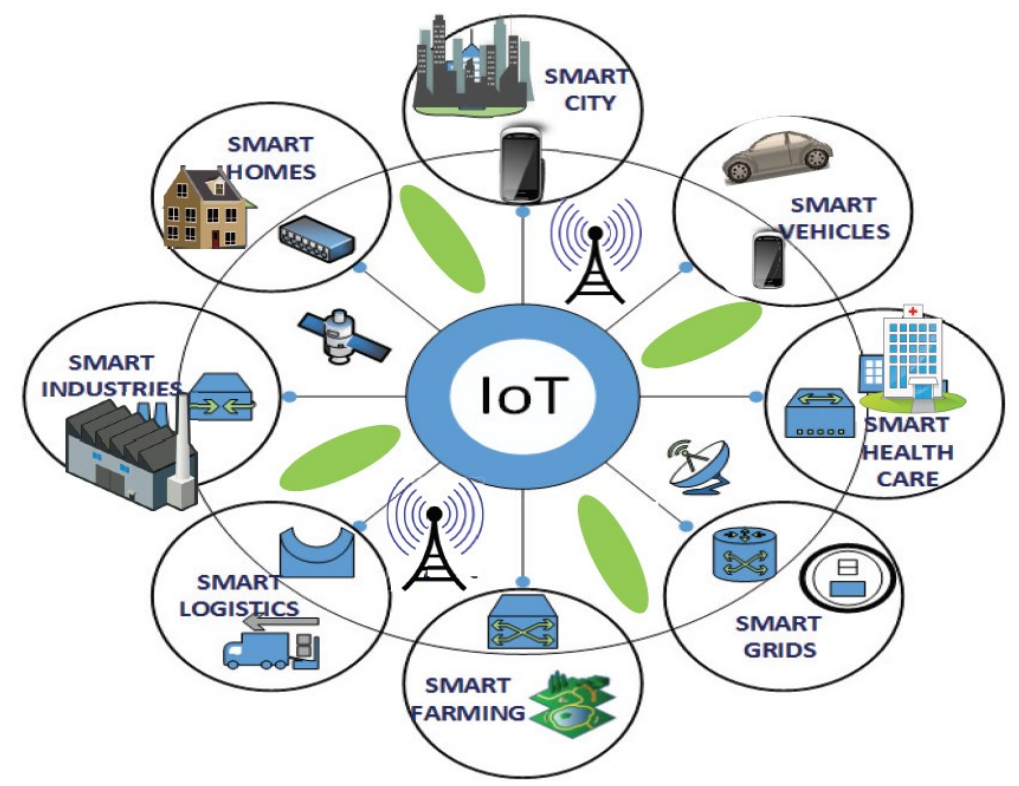

Figure 2.3. Applications of IoT [9]. 
Beam-steering capability, low-cost, ease of fabrication, and compactness are among the advantages of LWAs compared to phased array antennas [28]-[31]. The difficulty in controlling $\theta_{0}$ and $\Delta \theta$ individually, inherent frequency beam-squint of an LWA, and lower gain compared to the phased array antennas are among the disadvantages of LWAs. The beam-scanning in the LWA, as presented in Fig. 2.5, can be achieved through frequency, mechanical, or electronic beam-scanning.

Since the late 1950s, numerous studies on LWAs have been carried out. Waveguide and microstrip structures are widely used as the guided structures for realizing an LWA [28]-[31]. A microstrip structure has an inherently large dielectric loss. On the other hand, waveguides are bulky and not suitable for integration and miniaturization purposes. SIW [35]-[39] has been widely implemented to design LWAs mainly because of its integration capability [48]-[62].

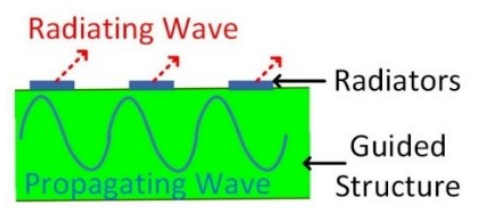

Figure 2.4. Leakage mechanism of an LWA.

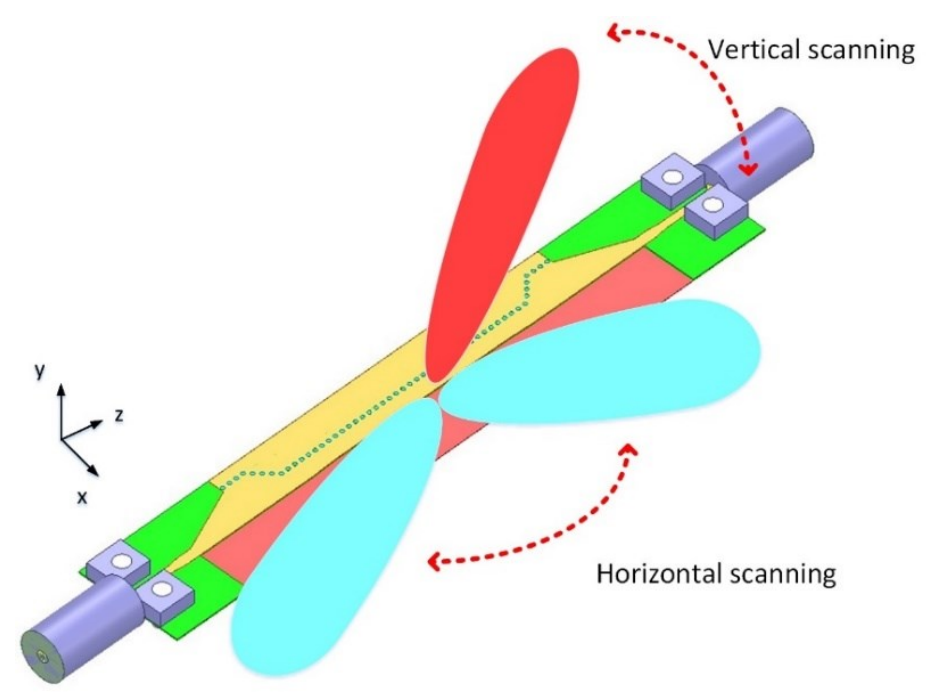

Figure2.5. Beam-scanning in an LWA. 
Dispersion response is defined as the behavior of the complex propagation constant throughout the operating frequency band [134]-[136]. A typical dispersion diagram, exhibiting several Floquet modes, is presented in Fig. 2.6. Floquet modes are also called space harmonics and occur due to the periodicity of a structure. As observed in Fig. 2.6, the higher-order Floquet modes are the shifted version of the fundamental mode $(n=0)$ [134], [135]. In a waveguide-based periodic structure, the phase constants of fundamental $\left(\beta_{0}\right)$ and higher order modes $\left(\beta_{n}\right)$ are calculated by

$$
\begin{gathered}
\beta_{n}=\beta_{0}+\frac{2 \pi n}{p} \\
\beta_{0} \cong k_{0} \sqrt{\varepsilon_{r}} \sqrt{1-\left(\frac{f_{c}}{f}\right)^{2}}
\end{gathered}
$$

where $\beta_{0}, n, p, \varepsilon_{\mathrm{r},} k_{0}$, and $f_{c}$ are phase constant of the fundamental Floquet mode, the number of the Floquet mode, period, relative permittivity, the free-space wavenumber, and cut-off frequency, respectively [134], [135]. Similar to the waveguide modes, Floquet modes also have cut-off frequencies that are determined by the period. In other words, the period of the cells determines the single mode propagation range. Often it is desired to have only one mode propagate. This can be accomplished by selecting the period, dielectric constant, and frequency range precisely [134]-[136]. In general, an LWA only radiates if it operates in the fast-wave region [28], [29], where $-k_{0}<\beta<k_{0}$. The fast-wave region is located between the airlines, as demonstrated in Fig. 2.6. In this region, the phase velocity of the leaky-wave is greater than the velocity of light in free space. The airlines with negative and positive slopes (i.e., $\beta= \pm \mathrm{k}_{0}$ ) represent backward and forward endfires, respectively. While the frequency axis represents the broadside (i.e., $\beta=0$ ). In the slow-wave region, the propagating wave is an evanescent 


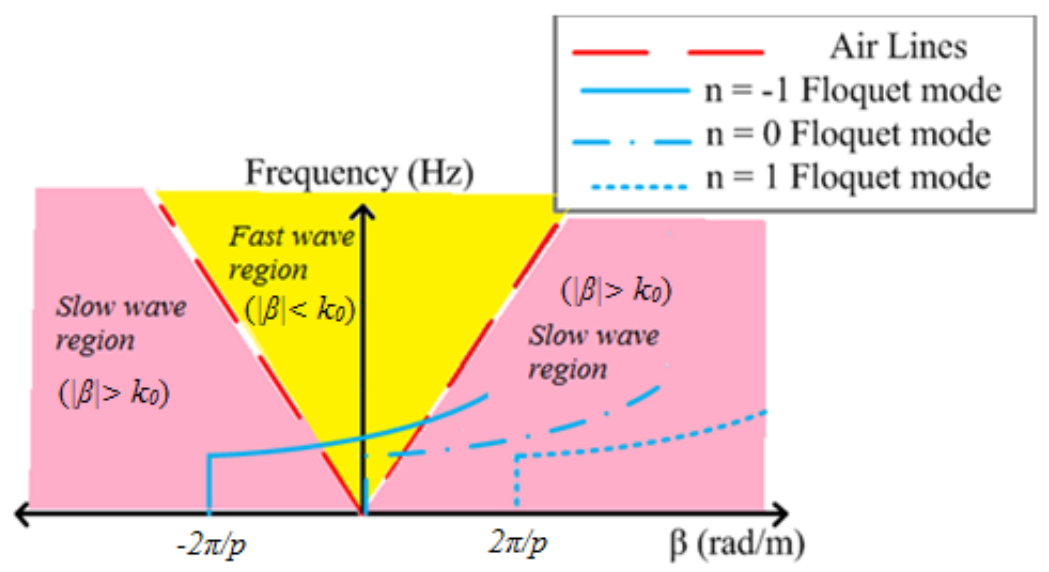

Figure 2.6. Dispersion diagram of a typical waveguide LWA.

wave, attenuating quickly. Hence, radiation does not occur when the wave is in the slowwave region.

The main-lobe pointing angle $\left(\theta_{0}\right)$ and the half-power beamwidth (HPBW) $(\Delta \theta)$ are calculated by

$$
\begin{gathered}
\cos \left(\theta_{0}\right)=\frac{\beta_{n}}{k_{0}} \\
\Delta \theta \cong \frac{\lambda_{0}}{L_{r} \sin \left(\theta_{0}\right)}
\end{gathered}
$$

where $L_{r}$ and $\lambda_{0}$ are the radiation length and the free-space wavelength, respectively [28], [29]. According to (2.3), beam-scanning occurs by changing the phase constant $\beta_{n}$, which lays the foundation of beam-scanning capability in an LWA. According to (2.3) and (2.4), the HPBW reduces by approaching the broadside region. On the other hand, approaching the endfire results in a larger HPBW [28], [29]. It should be noted that $\theta_{0}$ was measured from the forward endfire (i.e., $\theta_{0}=0^{\circ}$ is at the forward endfire).

LWAs are divided into three categories: uniform LWA (ULWA), quasi-uniform LWA (QLWA), and periodic LWA (PLWA) [28]-[30]. A ULWA has a uniform cross-section along the structure. A slot-based ULWA usually contains a single longitudinal long slot, as presented in Fig. 2.7(a). A ULWA cannot scan the whole hemisphere and only scans 
the forward quadrant, i.e., from broadside to forward endfire [28]-[30], [48]-[50] because only the fundamental Floquet mode $(n=0)$ propagates in the ULWA. The SLL of a ULWA can be reduced by tapering the via fence and the slot, as shown in Figs. 2.8 and 2.9 [48], [50]. The SLL was reduced to about $-40 \mathrm{~dB}$ [48] and to about $-23.2 \mathrm{~dB}$ [50] by tapering the via fence and shape of the slot.

A QLWA contains several closely-spaced radiating elements, as illustrated in Fig. 2.7(b). Since the period is so small, the cross-section can be considered uniform [28]-[30]. The small period of the slots assures that only the fundamental Floquet mode propagates. Hence, this antenna scans only the forward quadrant [28]-[30], [51]-[54]. A SIW-based QLWA with transversal slots was introduced in [51] for the first time, as shown in Fig. 2.10. In [51], tapered slots and via fences at both ends of the antenna were implemented to improve the return loss. The SLL of a QLWA can be reduced by tapering the via fence and length of the transverse slots, a type of amplitude tapering, as demonstrated in Figs. 2.11 and 2.12 [52], [54]. Tapering the slots in a shape of a fish led to a SLL reduction of about $20 \mathrm{~dB}$ [52], while tapering them in a shape of an eight-wing butterfly resulted in a SLL about $-14 \mathrm{~dB}$ [54]. Changing the period of transverse slots, a type of space tapering, leads to SLL reduction of about $20 \mathrm{~dB}$ as well [53], as presented in Fig. 2.13.

A PLWA has a periodic cross-section and contains several periodic radiating elements, as demonstrated in Fig. 2.7(c). Increasing the period of the cells results in the excitation of higher-order Floquet modes. As a result, a PLWA can scan the upper hemisphere i.e., from backward endfire to forward endfire [28]-[30], [55]-[57]. This is one of the main advantages of a PLWA. The radiation patterns of a PLWA and a QLWA 
can be partially controlled following the array theory. However, it should be noted that LWAs are inherently different than array antennas due to the leaky-wave nature and gradual leakage from the discontinuities [28], [29].

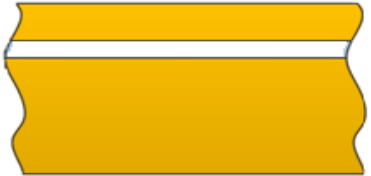

(a)

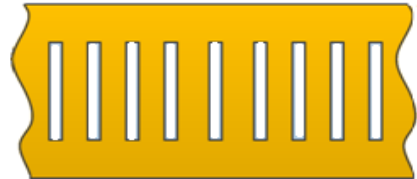

(b)

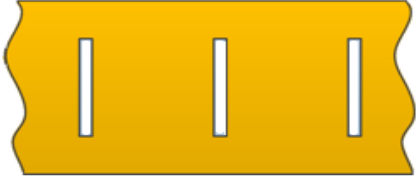

(c)

Figure 2.7. Schematic view of different types of an LWA. (a) ULWA, (b) QLWA, and (c) PLWA.
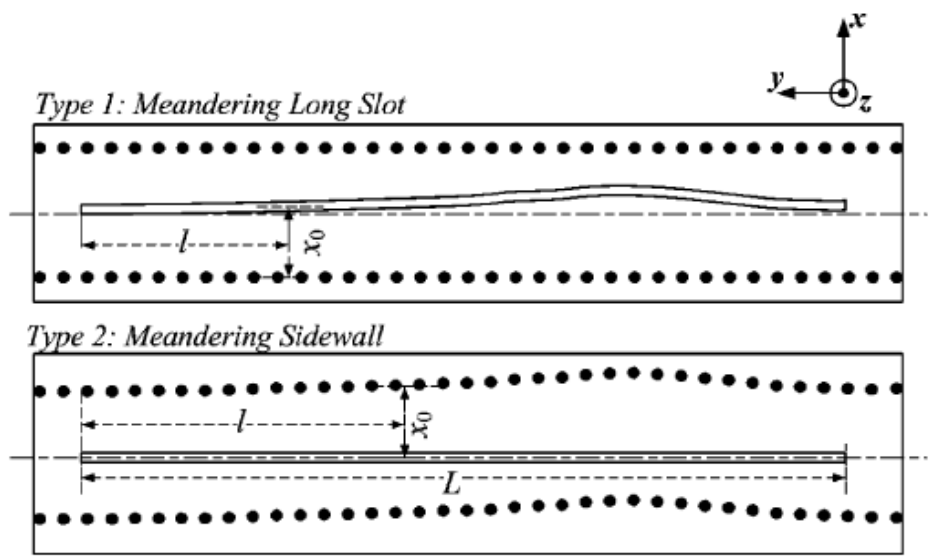

Figure 2.8. Proposed ULWA with reduced SLL in [48].

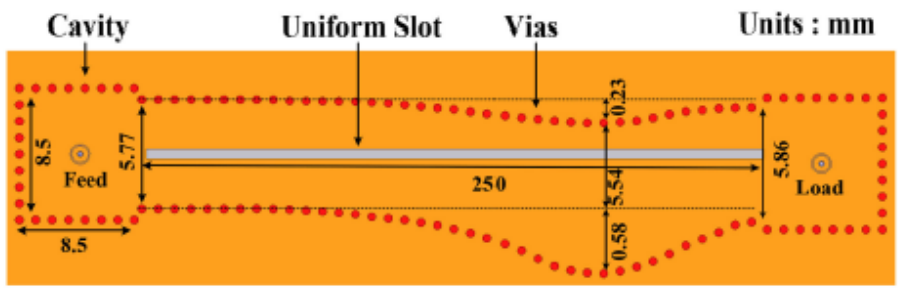

Figure 2.9. Top view of the proposed ULWA in [50].

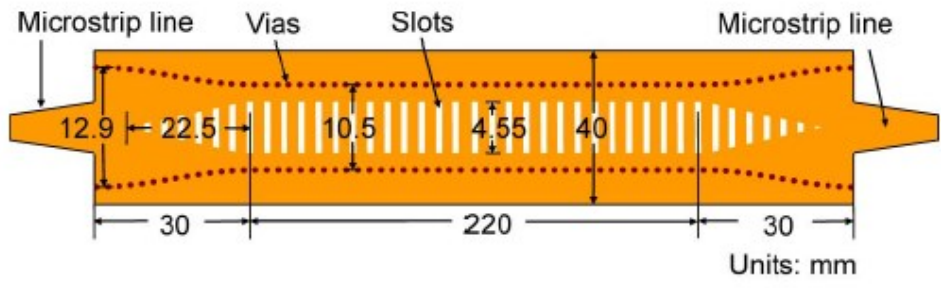

Figure 2.10. The first reported SIW QLWA [51]. 


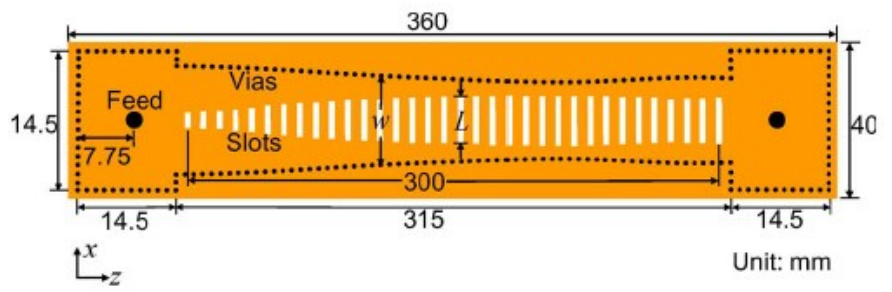

Figure 2.11. Tapered SIW QLWA with reduced SLL [52].

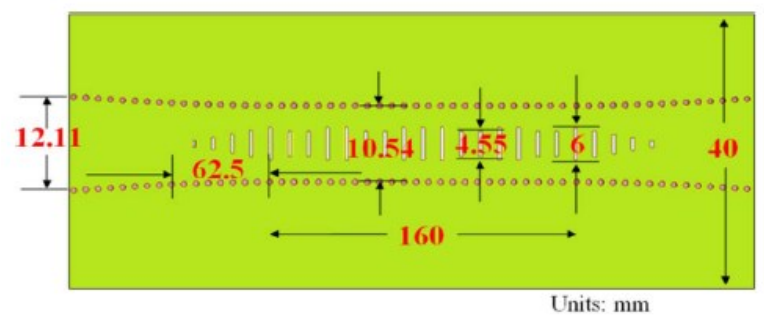

Figure 2.12. Butterfly-shape QLWA with reduced SLL [54].

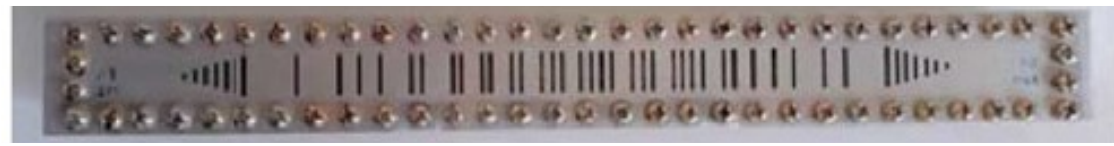

Figure 2.13. Thinned array QLWA based on air-filled waveguide [53].

It should be mentioned that the conventional PLWAs cannot scan the broadside due to the open-stop band (OSB) phenomenon [28]-[30], [44]-[46]. In a ULWA or QLWA, the beam is at broadside when no propagation occurs in the guiding structure, which is because of $f=f_{c}$ and $\beta=0$. In a PLWA, the travelling wave becomes a standing wave at broadside. Hence, all the reflections add in-phase, causing significant impedance mismatch. The antenna structure must be modified to enable the broadside scanning. It can be done by implementing periodic engineered structures called metamaterial cells, which will be discussed in Section 2.5. Introducing additional via posts adjacent to the slots can resolve the OSB issue. The mitigation of the OSB was achieved by matching the impedance of the slots and the via posts in each antenna when the beam is at broadside, as presented in Fig. 2.14 [45], [46]. The broadside scanning can be also achieved by implementing surface-wave launchers and strip lines to support surface-waves $[137]-[139]$. 


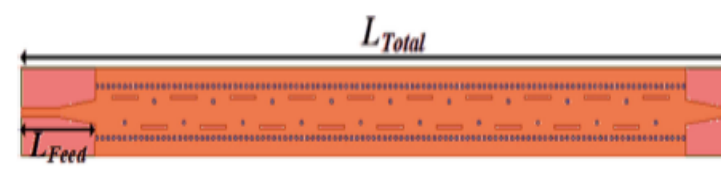

(a)

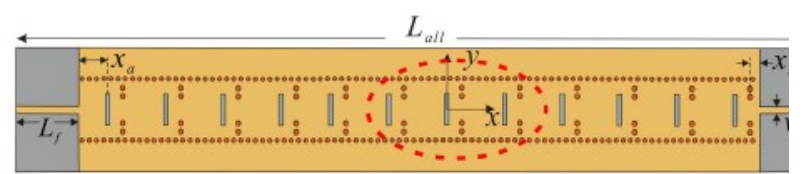

(b)

Figure 2.14. LWAs with additional via posts. (a) longitudinal slot [45], (b) transverse slots [46].

\subsection{Substrate Integrated Waveguide}

There is a constant push for complex integrated communication devices. Hence, the device footprint becomes a premium. The antenna can be designed based on SIW to achieve a low-profile structure with reasonable cost [35]-[43]. SIW is widely used in mm-wave applications due to its remarkable benefits such as ease of fabrication, integration capability, compactness, and low cost. Hence, SIW is a suitable candidate as the guiding structure of the LWA. SIW was introduced in [41]-[43] for the first time as demonstrated in Fig. 2.15. Placing posts in a parallel plate waveguide resulted in confinement of the fields, as observed in Fig. 2.15(b). Before introducing SIW, the transition from waveguide to microstrip line was through complex and bulky structures that were not suitable for integrated applications [43]. One of the key features of SIW is the integration of waveguide into a planar form [43].

Via holes in a SIW can be utilized to emulate the operation of a dielectric-filled rectangular waveguide [35]-[37], as illustrated in Fig. 2.16. The via fences are in the shape of cylindrical holes that are filled with a conductive resin such as silver paste. The via fences act as the sidewalls of the waveguide that confine the electric field of the $\mathrm{TE}_{\mathrm{n} 0}$ mode. In other words, SIW can only prevent $\mathrm{TE}_{\mathrm{n} 0}$ modes from leaking into space from the side via fences as the surface current of these modes is not disturbed by the vias. In contrast, the currents of $\mathrm{TM}$ and $\mathrm{TE} \mathrm{E}_{\mathrm{nm}} \mathrm{m} \neq 0$ are disturbed and leakage occurs from the side via fences for such modes [35]-[37]. 


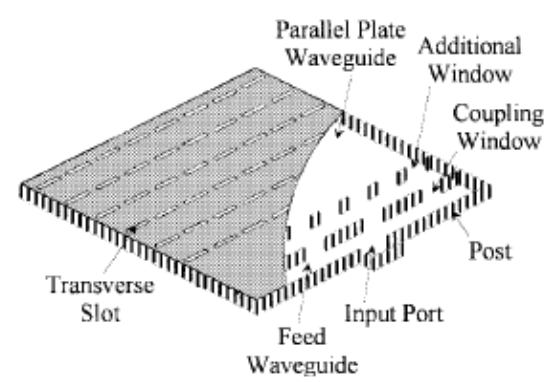

(a)

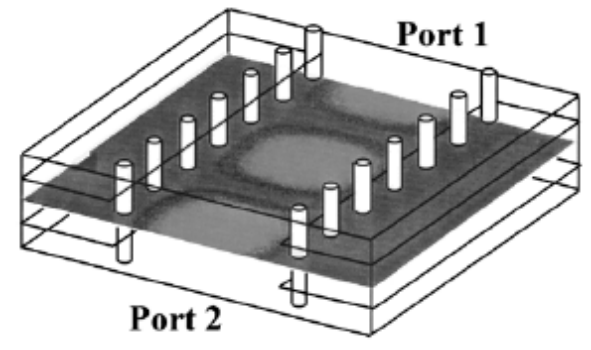

(b)

Figure 2.15. The first reported SIWs. (a) parallel plate waveguide with posts [42], (b) field distribution in [41].

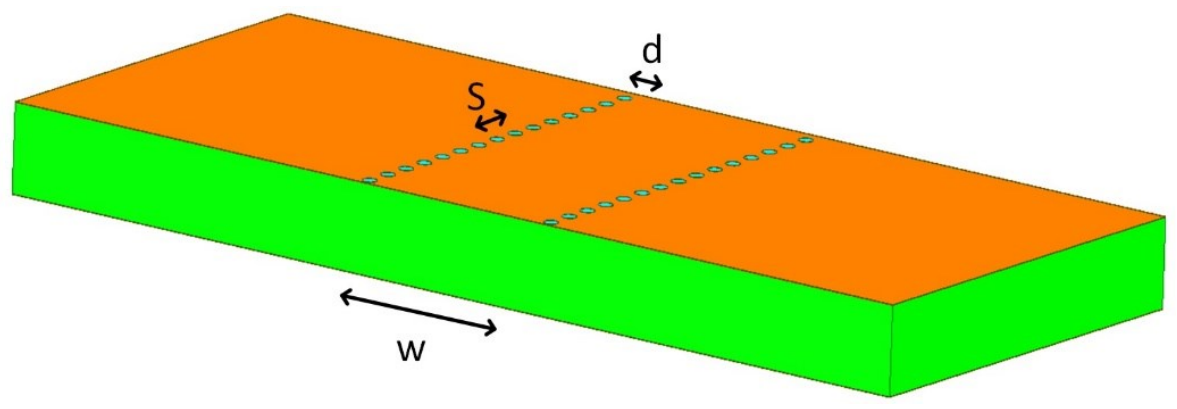

Figure 2.16. Conventional SIW.

Confinement of the electric field of the $\mathrm{TE}_{\mathrm{n} 0}$ modes in a SIW can be achieved by adjusting the diameters of the vias $(d)$ to be larger than one-third of the via spacing $(s)$ and smaller than one-fifth of the width (transversal spacing of the via fences) $(w)$ [35], [36]. The effective width of the structure $\left(w_{e f f}\right)$ and the cut-off frequency $\left(f_{c}\right)$ of $\mathrm{TE}_{\mathrm{n} 0}$ are calculated by

$$
\begin{gathered}
w_{e f f}=w-1.08 \frac{d^{2}}{s}+0.1 \frac{d^{2}}{w} \\
f_{c}=\frac{c}{2 w_{e f f} \sqrt{\varepsilon_{r}}}
\end{gathered}
$$

where $w_{e f f}, w, d, s, c$, and $\varepsilon_{r}$ are the effective width, width, via diameter, via spacing, the velocity of light, and permittivity, respectively [35], [140], [141]. Concerning dispersion characteristics, SIW has the same guided wave properties as a dielectric-filled rectangular waveguide [35]. Despite all the similarities among rectangular waveguides and SIWs, 
they have their differences. SIW is inherently a periodic structure that can suffer from the band-stop issue. Furthermore, if the via spacing is not set correctly, leakage can occur from the via fence due to the periodic gaps [35]. In terms of fabrication, if the conductive vias are not connected to the conductor planes through soldering, SIW will not confine the fields of $\mathrm{TE}_{\mathrm{n} 0}$ mode [35].

SIW can be fed using surface-mounted or through-hole connectors. Through-hole connectors are not suitable for thin substrate. Implementing surface-mounted connector requires a microstrip transition to transfer signal from coaxial connector to SIW. Unfortunately, the microstrip transition has undesired effects on the radiation pattern [142], which will be discussed in Section 3.3. It should be noted that despite the integration feature of the SIW, it is difficult to realize it at frequencies beyond $100 \mathrm{GHz}$ [82]. This is due to the fact that the spacing between the vias becomes too small at very high frequency which is a practical challenge [82].

Removing half of the top conducting cladding of SIW will make the structure more compact. This new structure is called half-mode substrate integrated waveguide (HMSIW) [63], shown in Fig. 2.17. In the HMSIW LWA, radiation occurs from both the side aperture [63] and the slots over the surfaces [67]. Since the size of the side aperture is larger than the slots, most of the leakage occurs from the side aperture. Hence, controlling the leakage mechanism in the HMSIW is more complicated than the SIW, which is one of the drawbacks of the HMSIW. 


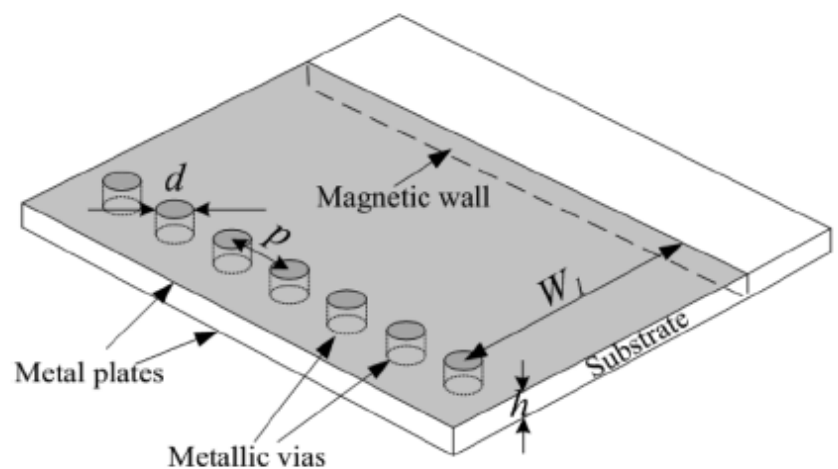

(a)

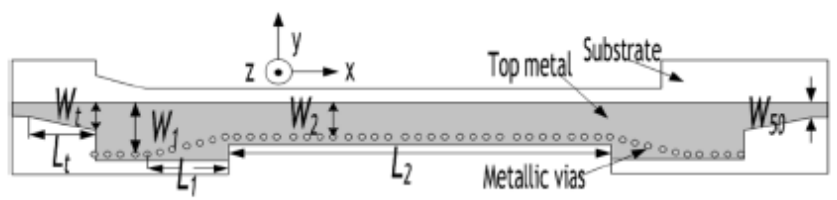

(b)

Figure 2.17. The first reported HMSIW. (a) expanded 3D view, (b) top view [63].

The field distribution of an HMSIW is illustrated in Fig. 2.18. The fundamental mode in HMSIW is $\mathrm{TE}_{0.5,0}$, not $\mathrm{TE}_{10}$, since half of the top conductor cladding is removed [76]. Hence, the cut-off frequency of the dominant mode in HMSIW is half of the corresponding value in SIW. In other words, the effective width of HMSIW is half of the effective width of similar SIW found from (2.6). The second mode is $\mathrm{TE}_{1.5,0}$ with the cut off frequency of three times the dominant mode $\left(\mathrm{TE}_{0.5,0}\right)$ cut-off frequency [76].

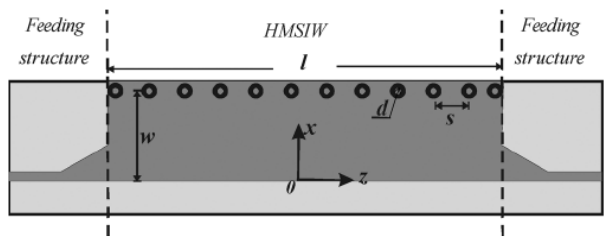

(a)

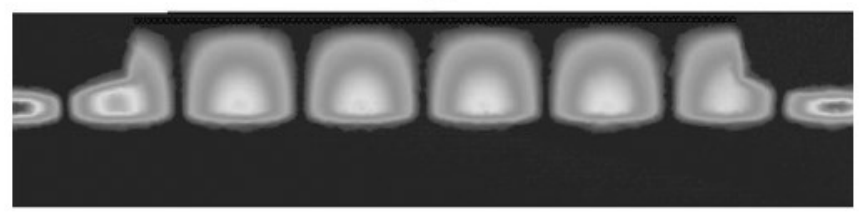

(b)

Figure 2.18. Conventional HMSIW (a) top view, (b) electric field distribution of the dominant mode (TE.0.5) in HMSIW [76]. 
An HMSIW LWA generally has two main beams in its radiation pattern due to the small ground plane and reflection from the aperture. By increasing the thickness of the substrate, the lobe level of the second main-lobe reduces [63], [76]. The SLL can be improved by tapering the via fence [64]-[66] and side aperture [69], [70]. In [65], the side via fence was divided into multiple equal-length small segments, as presented in Fig. 2.19(a). Then, using particle swamp optimization (PSO) and genetic algorithm (GA), the transversal location (i.e., distance from the central longitudinal axis) of each segment was optimized to minimize the SLL [65]. It was observed that if the phase constant varies significantly, the proposed method in [65] is not very useful for reducing the SLL. Unfortunately, the radiation underneath the HMSIW was ignored in [65]. While according to [63] and our investigation in Chapter 3, a large SLL exist in the lower hemisphere (i.e., underneath the HMSIW). Implementing the proposed method in [65] led to the reduction of SLL from $-10.4 \mathrm{~dB}$ to $-19.8 \mathrm{~dB}$.

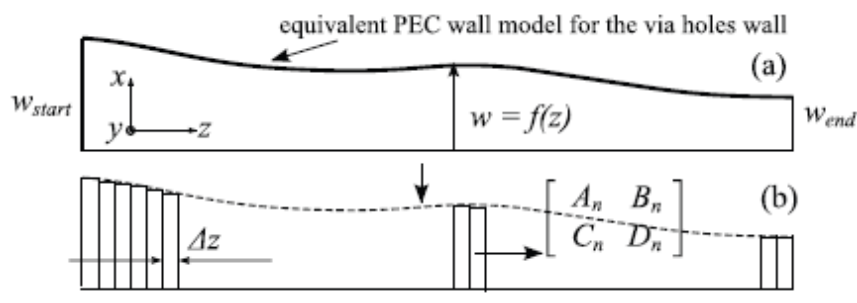

(a)

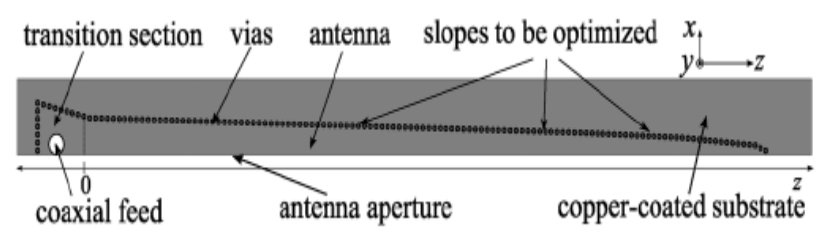

(b)

Figure 2.19. Proposed HMSIW LWA with tapered via fence for SLL reduction [65]. (a) the proposed method based on dividing the side via fence into multi-section [65], (b) schematic of the reported HMSIW antenna with reduced SLL [75]. 


\subsection{Low-Temperature Co-Fired Ceramic}

There is a continuous desire to miniaturize wireless devices. LTCC technology is among the most reliable and cost-effective technologies for implementing miniaturized designs [77]-[93]. LTCC was introduced in 1996 [78]. The packaging tolerance of LTCC structures is higher than PCBs since all layers in LTCC are fabricated simultaneously [77]-[79]. This is important in highly dense integrated applications. Overall, LTCC technology is a better candidate for miniaturized applications [78] due to its appealing properties, such as high packaging tolerance, ease of integration, the possibility of embedding components and cavities, low loss, and robustness to environmental conditions such as humidity [77]-[80]. However, surface roughness and thermal expansion of the layers are among the major issues in the LTCC design [77]-[79]. Furthermore, LTCC technology is more expensive than PCB.

LTCC is a multi-layer structure in which different dielectric tape layers are stacked-up on top of each other and sandwiched between the conductor planes [77]. Generally, silver and gold are used as conductive materials in the LTCC structure. Therefore, the conductor loss of the LTCC is low. Dupont9k7 and FerroA6M are the most popular choices for the dielectric tape layers with dielectric constants of 5.6 and 7.1, respectively [77], [78]. The loss tangent $(\tan \delta)$ of both is about 0.001 [77], [78]. Manufacturing the LTCC is a multi-step process [77], [78], as shown in Fig. 2.20. After stacking-up the tape layers over the conductor layers, the structure is baked. The baking/heating process results in the shrinkage of the layers [78]. To boost the thermal and mechanical strength, the bottom conductor layer must have meshed such that at most contains $50 \%$ conductive material [78]. 


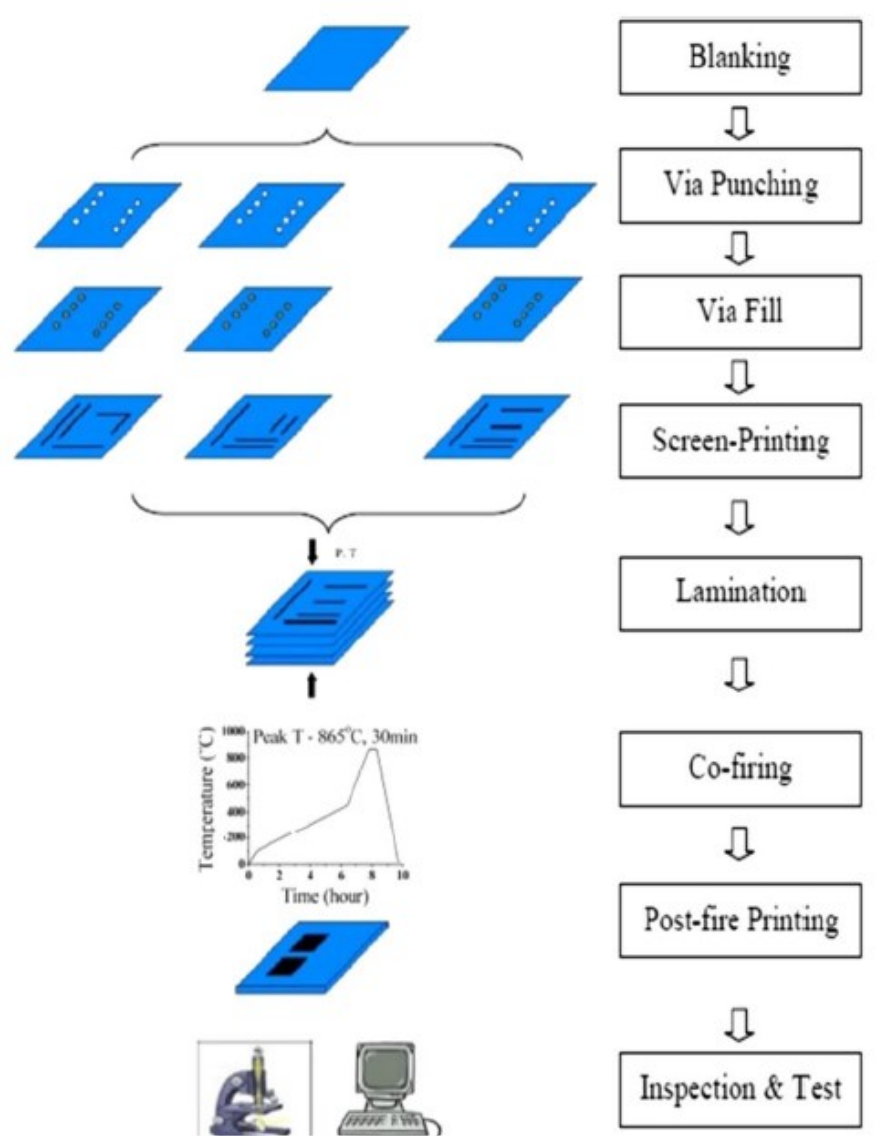

Figure 2.20. Fabrication procedure of LTCC [78].

The properties of LTCC are very appealing for the implementation of planar antennas at high frequency bands. Compactness, low loss, the possibility of ferroelectric integration, and beam-scanning capability are among the features that make LTCC based SIW LWA a suitable candidate for different mm-wave applications [78].

A few compact vertical guiding structures based on LTCC were introduced in [82] to reduce the footprint, as shown in Fig. 2.21. This is one of the advantages of LTCC technology. The proposed idea in [82] was obtained from Silicon-based IC design in which the reduction of footprint at the expense of increasing the thickness is more desirable. 


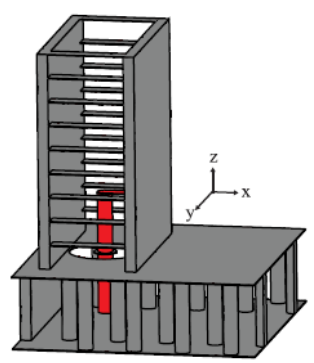

(a)

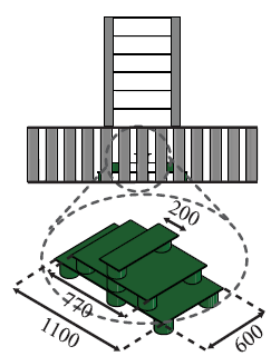

(b)

Figure 2.21. Vertical guiding structures based on LTCC [82]. (a) 3D view of the transition to SIW/horizontal waveguide, (b) 3D view of T-junction.

\subsection{Electronic Beam-Scanning}

Deployment of variable links require a beam-steerable antenna [1], [2]. Beamscanning can be achieved through frequency sweep, sweeping bias voltages of switches, or rotating the antenna mechanically. The electronic beam-scanning is the most preferable method due to its ease of implementation compared to the frequency and mechanical beam-scanning. Furthermore, using an electronic beam-scanning antenna can compensate for the unwanted frequency beam-squint in the face of variable operational and environmental conditions. Electronic beam-scanning can be accomplished by introducing active elements such as micro-electromechanical systems (MEMS) [143][146], semiconductor switches, varactor diodes, PiN diodes [127], ferrite switches, or liquid crystals [127], as shown in Fig. 2.22. Semiconductor switches and varactor diodes have the highest switching speed and are more compact than MEMS. A varactor diode can change the capacitance continuously by sweeping the bias voltage. PiN diodes have the smallest footprint, but provide limited switching states i.e., "on" and "off" states. MEMS are mechanically tunable switches [143]-[146], require high bias voltage, and usually bulkier than PiN diodes and varactor diodes. However, MEMS have more comprehensive capacitance tuning ranges than the varactor diodes. Liquid crystals are fairly lossy in mm-wave and not suitable for the integrated applications [127]. 


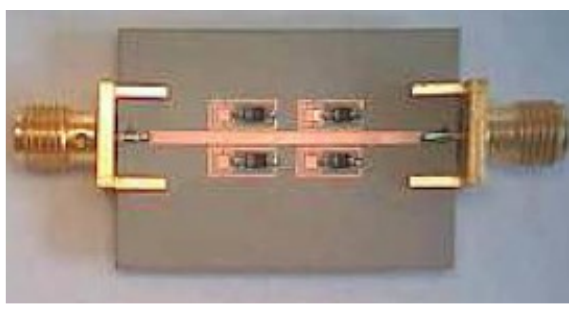

(a)

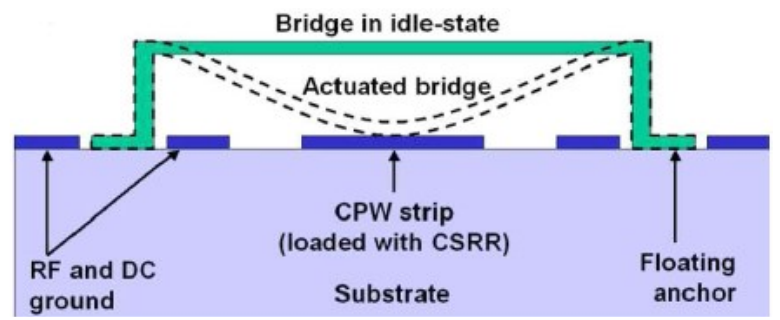

(b)

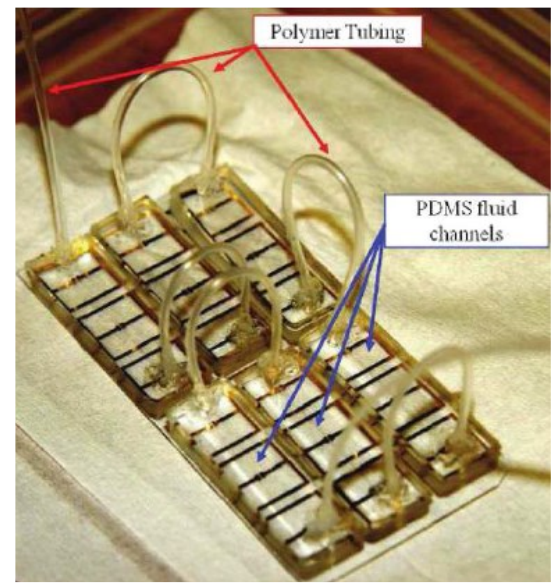

(c)

Figure 2.22. Different methods for achieving electronic beam-scanning [127]. (a) Using switches/varactor diodes, (b) Through MEMS, (c) Implementing liquid crystal.

A Gallium Arsenide (GaAs) varactor diode is a popular switch widely used in reconfigurable structures. Small footprint, high switching speed, ease of integration, and robustness are among the benefits of GaAs switches [127]. Hence, in the proposed designs, GaAs varactor diodes are realized to function as switches for electronic beamscanning. Tuning the bias voltages can be done manually or automatically through FPGAs and microcontrollers. Only a few commercial GaAs varactor diodes operate in mm-wave frequency bands. In this dissertation, MAVR000120-14110 [147] and 
MAVR011020-14110 [148] varactor diodes are chosen as the required switches in the reported electronic beam-scanning antennas. The capacitance ranges of these varactor diodes are $0.2-0.9 \mathrm{pF}$ [147] and $0.03-0.19 \mathrm{pF}$ [148], respectively. The corresponding reverse bias voltage ranges from $1 \mathrm{~V}$ to $15 \mathrm{~V}$.

Several types of electronic beam-scanning antennas are reported in the literature, which are presented here briefly. Reconfigurable microstrip patch array antennas called "pixel antennas" were introduced [122]-[124]. In pixel antenna, several patches were connected through sets of switches, as presented in Fig. 2.23. Tuning the bias voltages of the switches led to variations in the antenna topology. A pixel antenna often requires implementing a large number of switches that increases the cost, complexity, and loss of the structure [123]. Implementing patches with different sizes [123] mitigates this issue to some extent. It should be noted that at high frequencies, the dimension of the switch is not negligible. Hence, the footprints of the switches affected the radiation pattern undesirably. The undesired effect of the switch on the radiation pattern can be reduced by placing the switch on the backside of the antenna.

Reconfigurable metamaterials are widely used to achieve tunable refractive index and phase manipulation. Cloaking, tunable absorbers, tunable filters, tunable power dividers, and electronic beam-scanning antennas are among the other applications of reconfigurable metamaterials [127]. Metamaterial structures are artificially engineered to achieve unnatural responses that are difficult to achieve using conventional architectures [149]. 


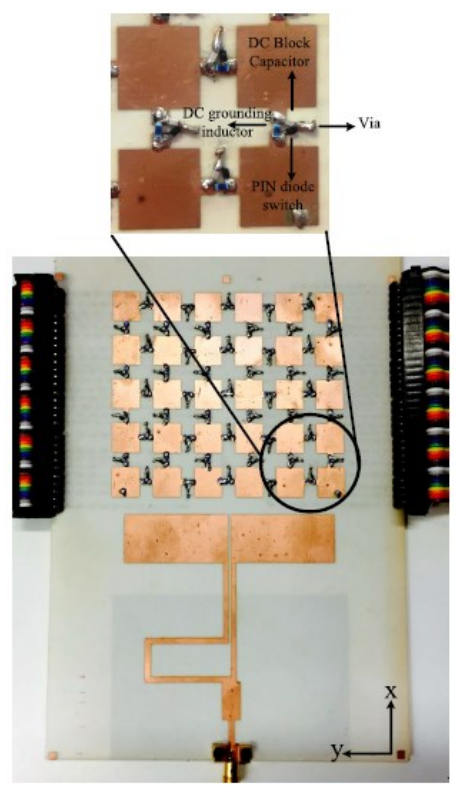

(a)

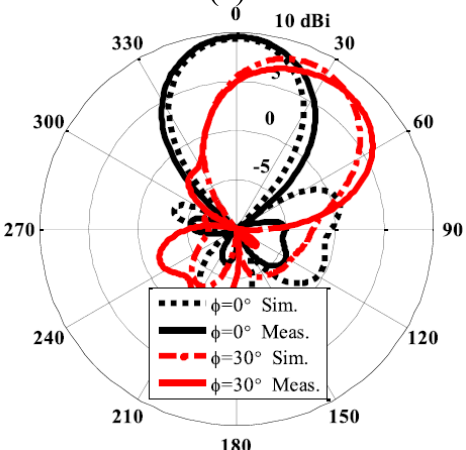

(c)

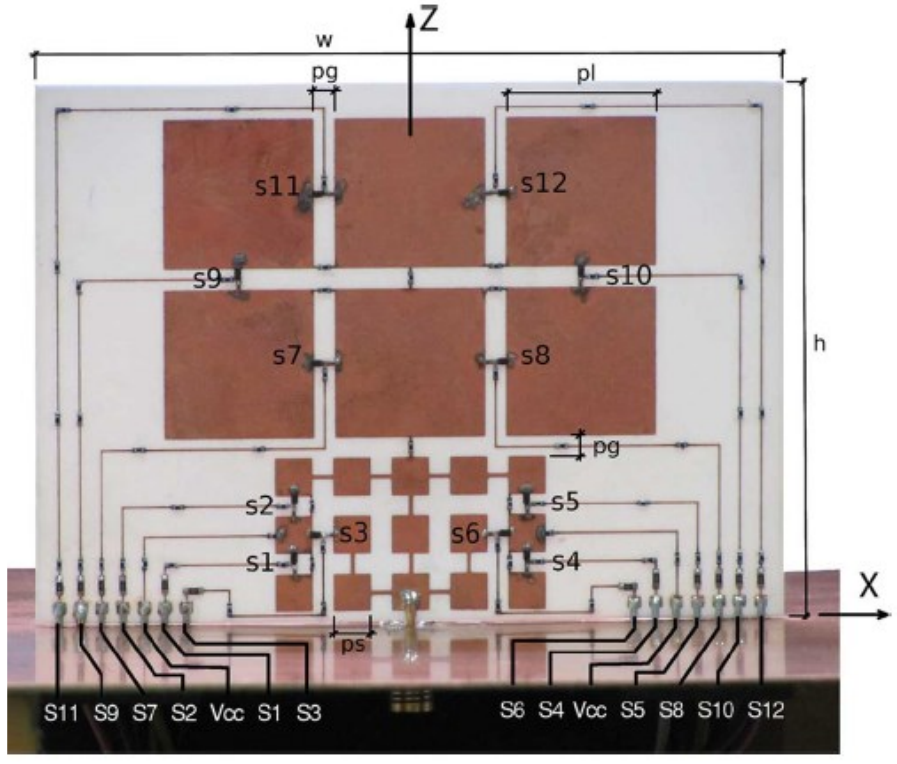

(b)

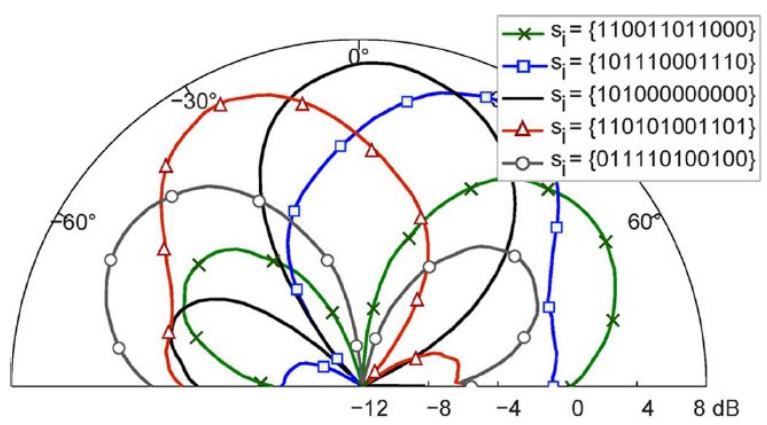

(d)

Figure 2.23. Pixel antennas. (a) fabricated antenna with equal-sized patches [122], (b) fabricated antenna with different sizes patches [123], (c) radiation pattern at $2.5 \mathrm{GHz}$ [122], (d) measured radiation pattern at $2.45 \mathrm{GHz}[123]$.

One of the most famous metamaterial structures is the composite right-hand left-hand (CRLH) structure [149]-[165]. The equivalent circuit of a CRLH structure is shown in Fig. 2.24(a), consisting of shunt and series resonators [149]. To realize a CRLH structure, shunt inductors and series capacitors must be added to the conventional transmission line that contains shunt capacitors and series inductors [149]. In a reconfigurable HM/SIW CRLH, as illustrated in Fig. 2.24(b), the top cladding, switches, substrate, and the via fences act as a series inductor, series capacitors, shunt capacitor, and shunt inductors, 
respectively. In a multi-layer structure, interdigital slots and embedded patches can also be modelled as series and shunt capacitors, respectively. The conventional structure (i.e., RH structure) has positive permittivity and permeability and supports forward radiation. In contrast, the LH structure has negative permittivity and permeability and supports backward wave propagation. CRLH structure supports both backward and forward radiation depending on the frequency band. Hence, CRLH LWA scans from backward endfire to forward endfire. As stated before, implementing CRLH is a solution for overcoming the OSB issue that only happens if the balance conditions are satisfied [149]-[151]. In other words, shunt and series resonance frequencies in addition to the series and shunt resonance impedance must be equal [149]-[151]. If the balance condition is not satisfied, a gap appears in the dispersion diagram. The dispersion diagrams of the balanced and un-balanced CRLH structures are presented by dashed and solid lines in Fig. 2.25, respectively.

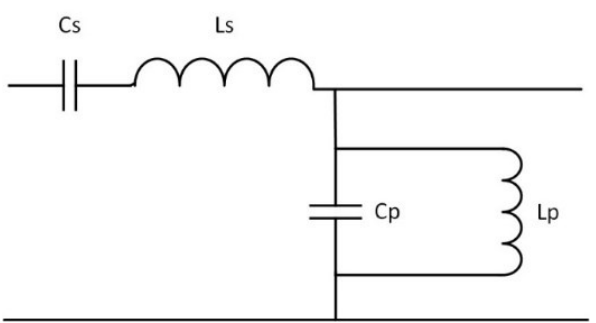

(a)

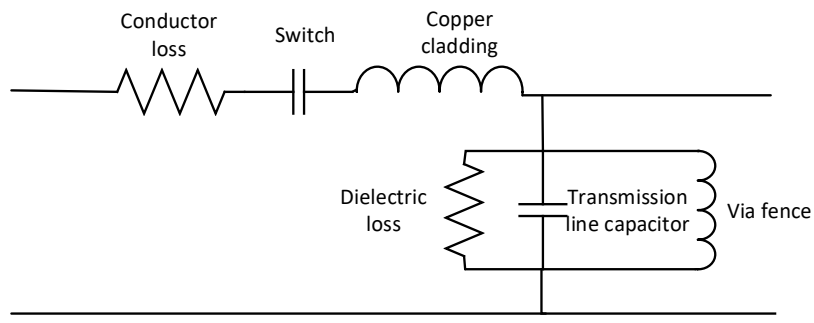

(b)

Figure 2.24. Equivalent circuit of CRLH. (a) conventional CRLH structure, (b) SIW-based CRLH structure.

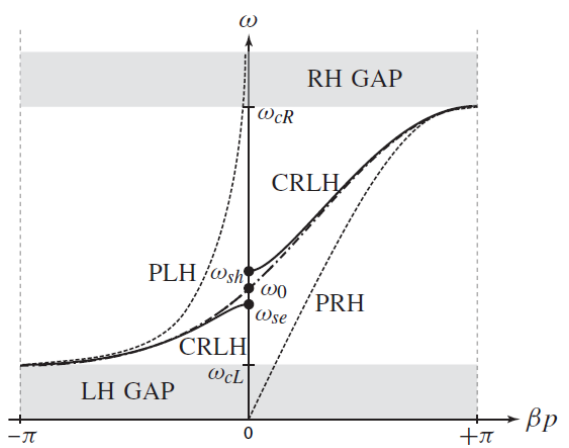

Figure 2.25. Dispersion diagram of a CRLH structure [149]. 
A CRLH structure is a suitable candidate for reconfigurable metamaterial antenna since changing the dimensions of CRLH cells leads to variations in permittivity and permeability [149]. The proposed antennas in [128] and [129] were among the first reported CRLH reconfigurable LWAs, as shown in Fig. 2.26. Sweeping the bias voltage led to variations in the capacitance of the varactor diode. This led to the variations in the impedance and propagation constant, which caused the changes in the radiation pattern. Tuning the capacitance of all varactor diodes uniformly resulted in beam-scanning, while non-uniform tuning led to the variations in HPBW [129]. Both series and shunt varactor diodes were used in [129] to achieve a more flexible design approach. The antenna's beam scanned space from $-49^{0}$ to $50^{\circ}$ at $3.33 \mathrm{GHz}[128]$ and from $-10^{0}$ to $7.5^{\circ}$ at 3.23 $\mathrm{GHz}[129]$.

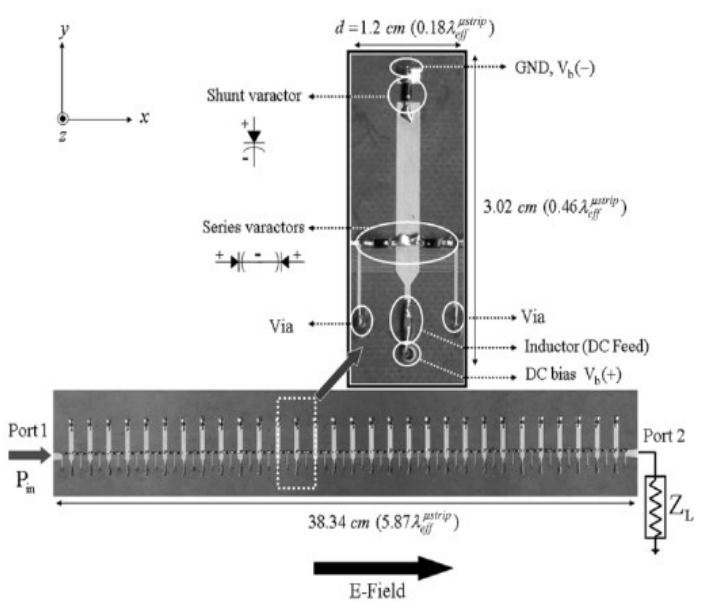

(a)

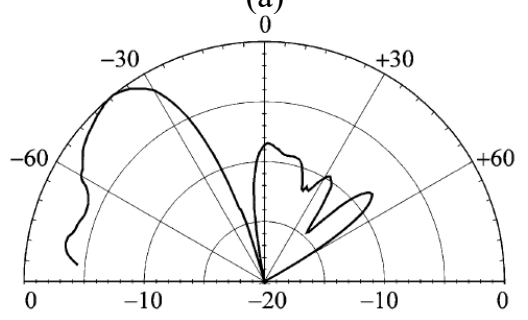

(c)

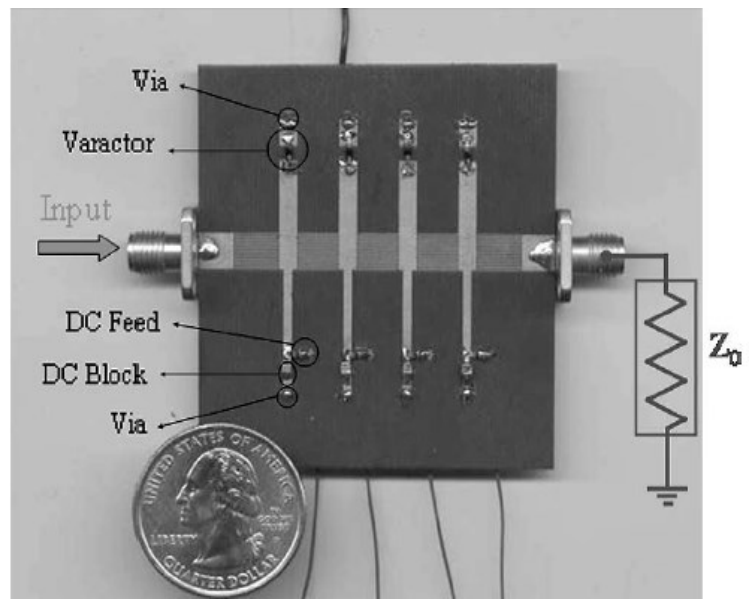

(b)

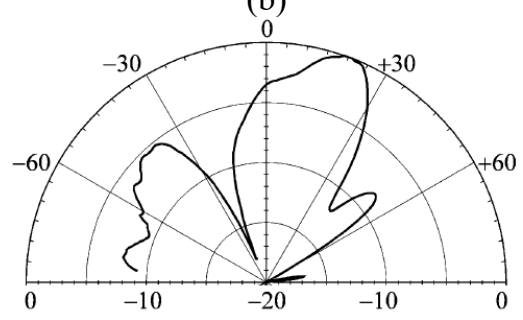

(d)

Figure 2.26. Reconfigurable CRLH antennas with shoring stubs and interdigital capacitors. (a)

fabricated antenna proposed in [128], (b) fabricated structure designed in [129], (c) radiation pattern at $18 \mathrm{~V}$ [128], (d) radiation pattern at $2 \mathrm{~V}$ [128]. 
HMSIW is a more suitable candidate for realizing a reconfigurable CRLH antenna than SIW since the shunt branches of SIW are usually not accessible [99]. A reconfigurable HHMSIW CRLH LWA was proposed in [99], as presented in Fig. 2.27. The proposed antenna made use of series and shunt tuning capacitors to achieve electronic beam-steering from $-31^{0}$ to $35^{0}$ at $6.5 \mathrm{GHz}$ with a peak gain of $9.5 \mathrm{dBi}$. In a multi-layer structure, interdigital capacitors and embedded patches can be used as series and shunt capacitors, respectively. Furthermore, the capacitors between the top cladding and grounded patches adjacent to the side aperture acted as shunt capacitors [99].

Placing several vias near the aperture of HMSIW, creating gaps around the vias in the ground plane, and connecting the via to the ground plane through switches also led to a reconfigurable HMSIW antenna, as shown in Fig. 2.28 [97]. Introducing 24 switches over the gaps in the ground plane led to the electronic beam-scanning from $31^{0}$ to $60^{\circ}$ at 6 GHz with a peak gain of $12.9 \pm 0.6 \mathrm{dBi}$ [97]. It was also observed that increasing the number of reconfigurable cells resulted in a broader scanning range [97].

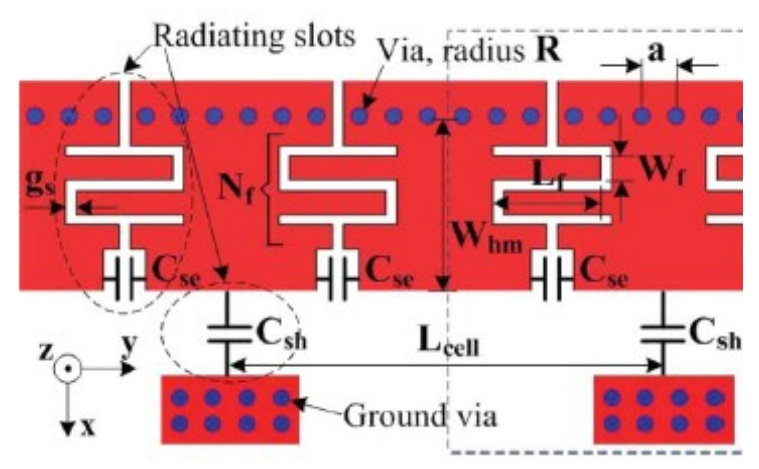

(a)

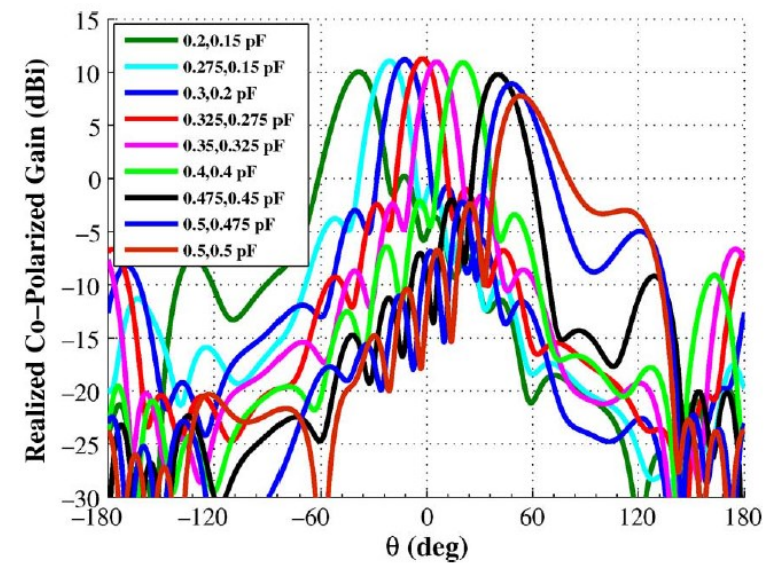

(b)

Figure 2.27. Reconfigurable CRLH HMSIW LWA [99]. (a) schematic of the antenna, (b) radiation pattern at $6.5 \mathrm{GHz}$. 


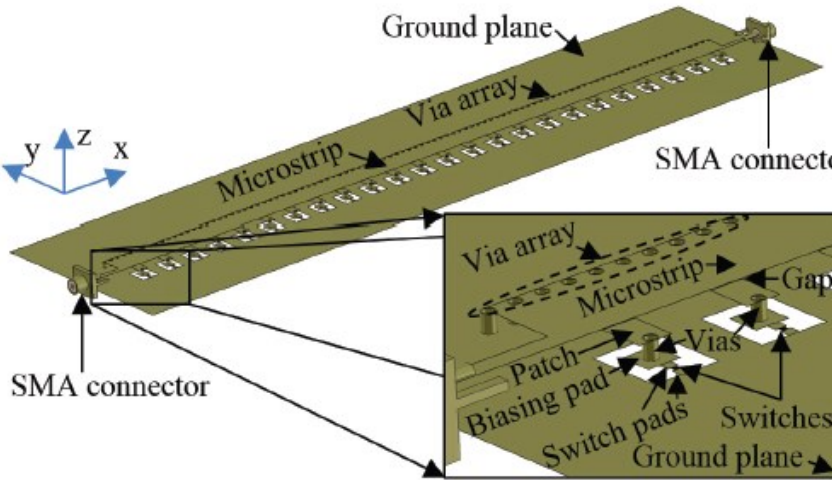

(a)

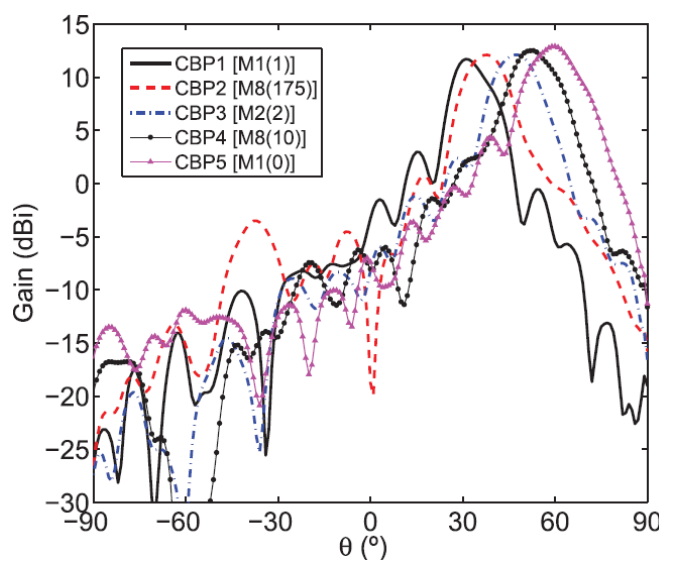

(b)

Figure 2.28. Reconfigurable HMSIW LWA with tunable grounded aperture [97] (a) schematic of the antenna, (b) radiation pattern at $6 \mathrm{GHz}$.

A reconfigurable HMSIW antenna with variable aperture can also be formed by placing several vias adjacent to the side aperture of HMSIW and connecting them to the aperture through sets of switches, as presented in Fig. 2.29 [104].

Placing a gap around each via of SIW and connecting the vias to the top cladding and ground plane through PiN diodes resulted in a reconfigurable SIW antenna [108], [111]. Changing the diode state ("on" or "off") led to the variations in the width of SIW. This changes the cutoff frequency and propagation constant, which led to the electric beamscanning from $46^{\circ}$ to $68^{\circ}$ at $5.2 \mathrm{GHz}$, as shown in Fig. 2.30 [108].

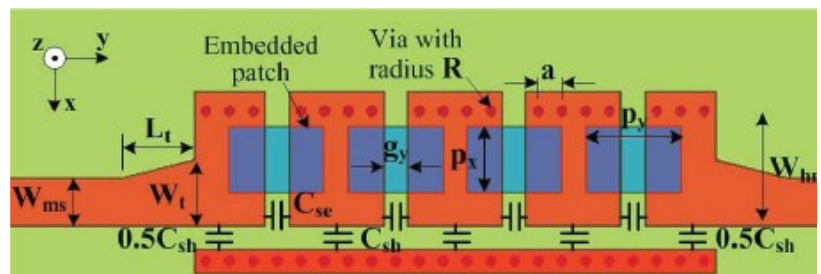

(a)

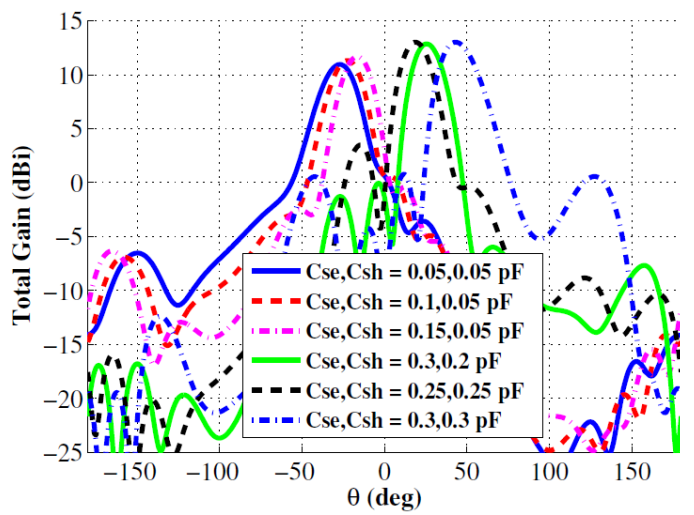

(b)

Figure 2.29. Reconfigurable CRLH HMSIW LWA with tunable grounded aperture [104] (a)

schematic of the antenna, (b) radiation pattern at $8.2 \mathrm{GHz}$. 


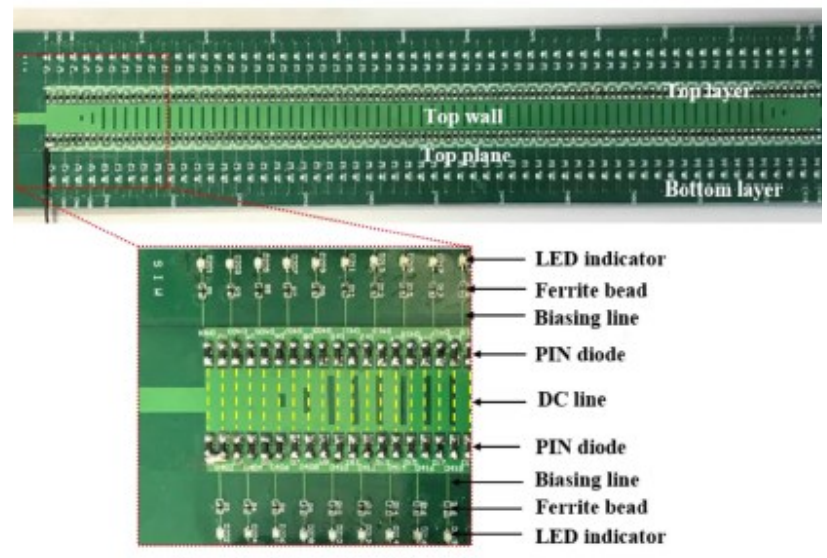

(a)

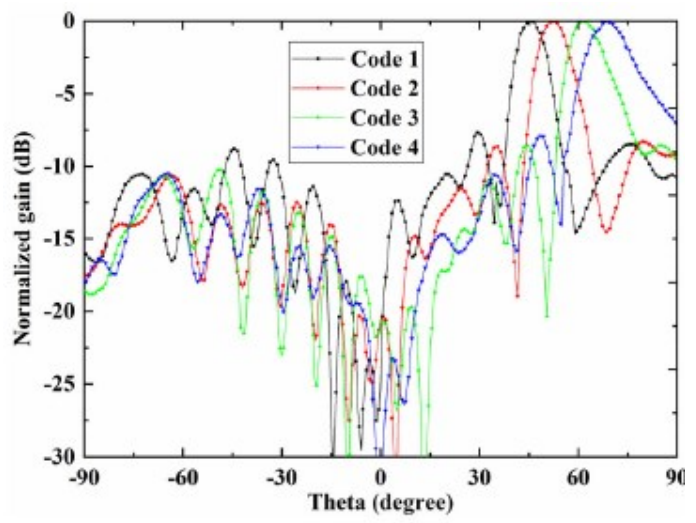

(b)

Figure 2.30. Reconfigurable SIW LWA with variable via fences [108]. (a) schematic of the antenna,

(b) radiation pattern at $5.2 \mathrm{GHz}$.

A reconfigurable corrugated SIW LWA was proposed in [109], as illustrated in Fig. 2.31. Corrugating the SIW helped with reducing the size of the structure. Switching the diodes led to the electric beam-scanning. The antenna scanned from $34^{\circ}$ to $59^{\circ}$ at $5.8 \mathrm{GHz}$ with a peak gain of $12.4 \mathrm{dBi}[109]$.

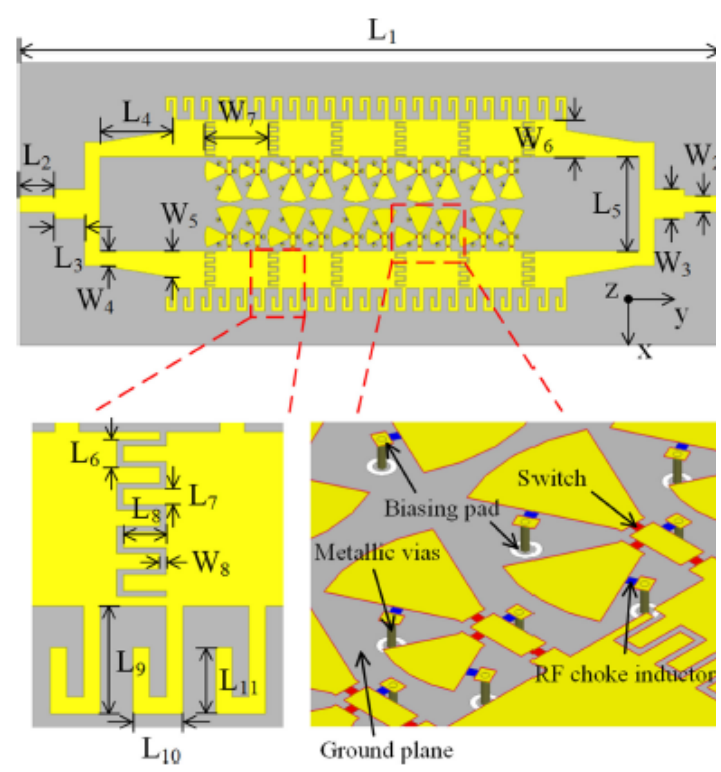

(a)

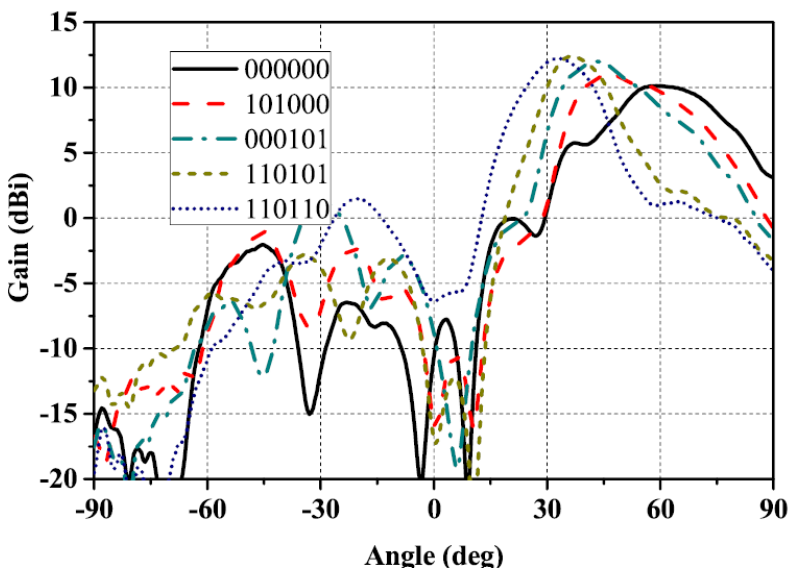

(b)

Figure 2.31. Reconfigurable corrugated SIW LWA with fan shape patches and open circuit stubs [109]. (a) schematic of the designed antenna, (b) radiation pattern at 5.8 GHz. 
A reconfigurable SIW cavity-backed antenna was introduced in [111], as presented in Fig. 2.32. Placing varactor diodes over the top and bottom gaps around the cylindrical posts led to the variation in the impedance and the propagation constant that resulted in electronic beam-scanning from $-30^{\circ}$ to $41^{\circ}$ at $2.6 \mathrm{GHz}$ [111].

For the first time, the via fences of a SIW were replaced by periodic cells with negative permittivity in [120], as shown in Fig. 2.33. It was shown that the cells confined the electric field of $\mathrm{TE}_{\mathrm{n} 0}$ modes. Hence, they could be used instead of the via fences. The proposed periodic cells were metallic posts with square shape rings. The permittivity and the permeability of the periodic cells were obtained using Nicolson Ross Weir (NRW) algorithm [121]. The proposed reconfigurable cells posed variable permittivity. Hence, an array of reconfigurable cells resulted in a reconfigurable structure.

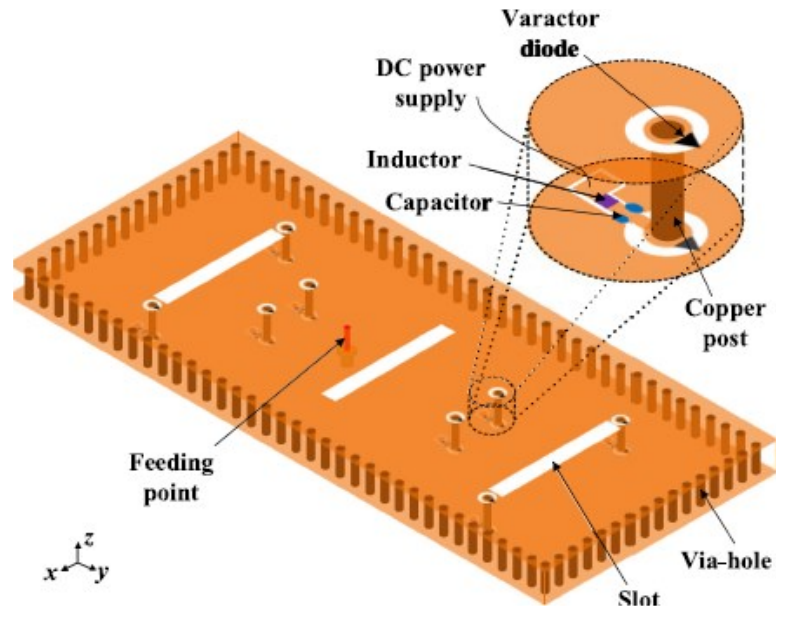

(a)

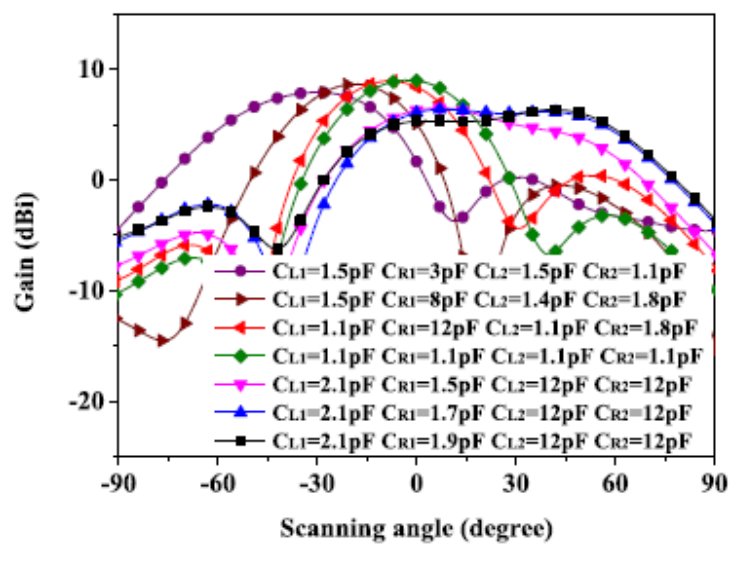

(b)

Figure 2.32. Reconfigurable cavity-backed SIW antenna with tunable posts [111]. (a) schematic of the antenna, (b) radiation pattern of the antenna at $2.6 \mathrm{GHz}$. 


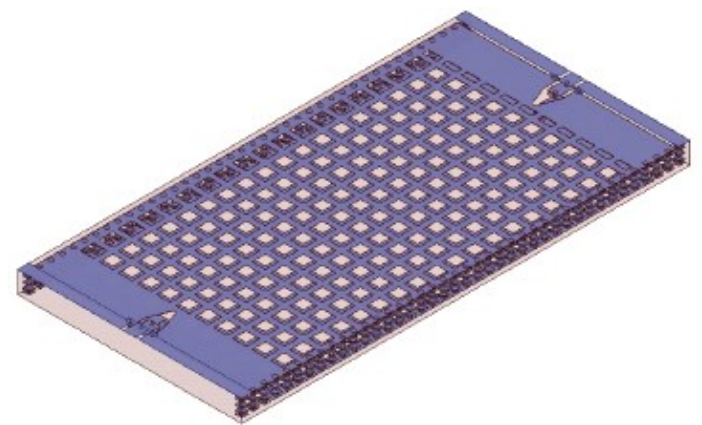

Figure 2.33. Schematic view of the proposed reconfigurable structure with negative $\varepsilon_{r}$ cells [120].

A reconfigurable microstrip structure called field-programmable microwave substrate (FPMS) was proposed in [121], as presented in Fig. 2.34. Different reconfigurable structures such as a waveguide, an oscillator, and an amplifier were realized based on FPMS, as shown in Fig. 2.35. The proposed structure has the potential to be used as a reconfigurable antenna. It contained 256 reconfigurable cells fabricated on PCB. As demonstrated in Fig. 2.34(a), the proposed cell consisted of cross-shape metallic stubs connected to a tapered patch through a varactor diode [121]. The bias circuitry was located underneath the structure. To bias the varactor diode, one end of the switch was connected to the ground through an inductor and the other end to the bias circuitry through an RF block (10k $\Omega$ resistor) and vias. To prevent large reflection of RF signal entering the switch, each varactor diode was grounded through a DC block $(2.2 \mathrm{nF}$ capacitor) and sets of vias [121]. Overall, some portion of the incoming signal went to the ground through varactor diode and DC block, while the rest passed to other cells through the stubs. Switching led to the variations in S-parameters that changed permittivity and permeability [121]. By applying the appropriate bias voltage, negative permittivity was achieved. Periodic cells with negative permittivity were implemented to confine the electric field. The proposed cells suffered from high loss due to the implementation of switches and RF blocks. However, it was discovered that reducing the cell size led to 
more negative permittivity with lower loss. Hence, smaller cells confine the electric field more efficiently [121]. It was observed that loss increased by approaching the resonance frequency of the cell. The proposed periodic cells were designed for the frequency band of $0.5-3.5 \mathrm{GHz}$ [121]. To achieve a miniaturized reconfigurable structure, reconfigurable cells were also designed in LTCC. The proposed LTCC structure is presented in Fig. 2.36 [121]. Each cell consisted of metallic parallel plate square rings with a central post. To reduce the number of switches, twelve cells were combined to create a master cell [121]. Each master cell was controlled by a CMOS chip. Different LTCC based waveguides were designed using the LTCC cells in the frequency bands of 2.5-3.5 GHz [121].

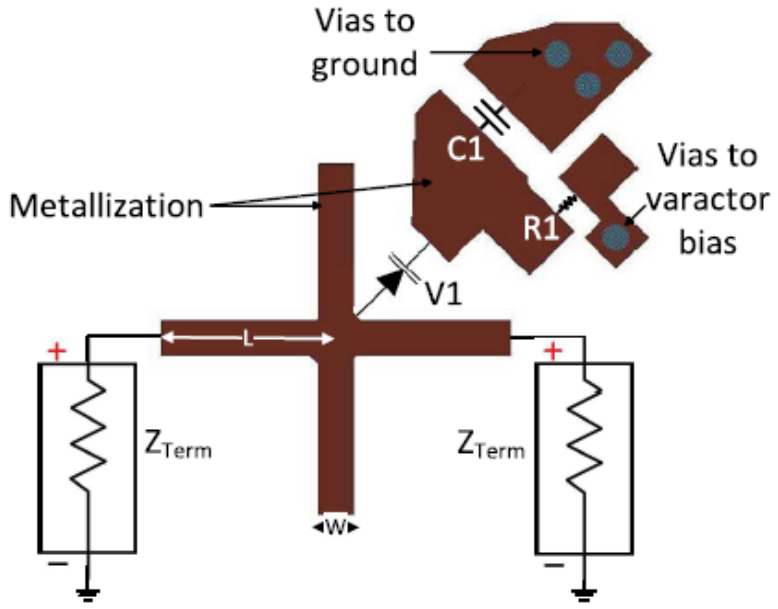

(a)

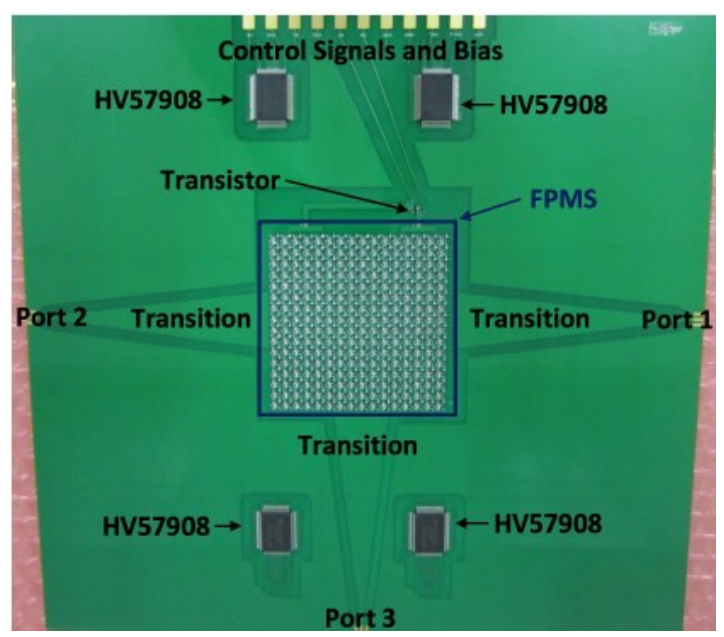

(b)

Figure 2.34. Schematic view of the field-programmable microwave substrate (FPMS) [121]. (a) the proposed unit cell, (b) FPMS.

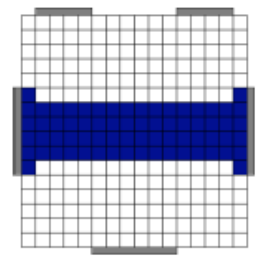

(a)

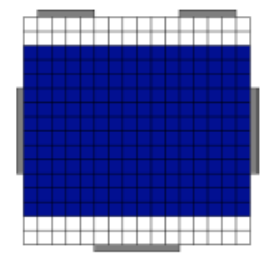

(b)

Figure 2.35. Reconfigurable waveguide realized on FPMS [121]. (a) $V_{\text {bias }}=25 \mathrm{~V}$, (b) $\mathrm{V}_{\text {bias }}=\mathbf{1 0 . 7 2} \mathrm{V}$. 


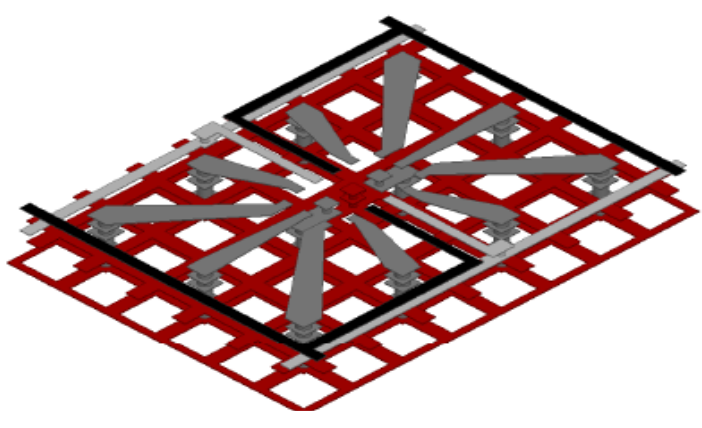

(a)

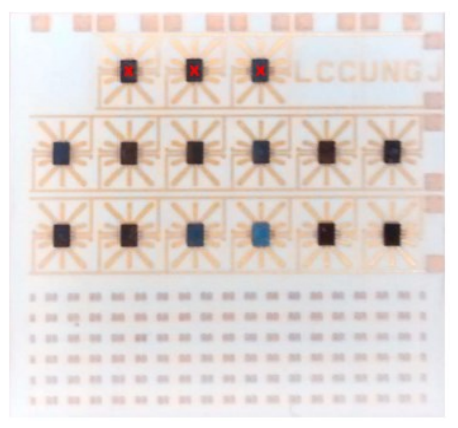

(b)

Figure 2.36. Schematic view of LTCC-based reconfigurable structure [121]. (a) master cell, (b)

\section{LTCC FPMS.}

Unfortunately, based on our investigations, achieving negative permittivity using the proposed cells for confining the field in mm-wave frequency bands is not possible. According to our studies, cells with $\varepsilon_{r}$ below -10 confine the electric field. Furthermore, cell size must be smaller than $\lambda / 10$ to achieve such permittivity. However, in mm-wave frequencies such as $28.5 \mathrm{GHz}$, fabricating such small cells and assembling the varactor diodes, chip capacitors, and chip resistors are not realistic.

\subsection{Summary}

In this chapter, the trends and the challenges of 5G applications were discussed first. Next, the fundamentals of LWA, SIW, and HMSIW were investigated. Different SIW/HMSIW-based LWAs were also introduced. LTCC technology along with its appealing features such as high packaging tolerance was introduced next. Finally, the foundations of electronic beam-scanning antennas and some exemplary ones were investigated.

This literature review provides a glimpse of the current challenges in antennas for 5G applications. It also shows the benefits and drawbacks of LWAs, SIW/HMSIWs, and reconfigurable antennas. While the advantages of the reported works confirm the suitability of SIW/HMSIW based LWA for 5G applications, their disadvantages shed 
light on possible areas that need further research and contributions. Reducing SLL, improving gain, and increasing beam-scanning range of a compact SIW/HMSIW LWA operating in mm-wave are among the crucial topics that need further investigations. In the following chapters of this dissertation, methods for reducing SLL, increasing gain, and achieving desired beam-scanning range will be proposed. 


\section{Chapter 3: Side-Lobe Level Reduction in HMSIW/SIW}

\section{LWAs}

To increase the detection accuracy and robustness to interference, the implemented antennas must scan space with a low SLL. Two design methods are proposed in this chapter to reduce the SLL of LWAs; one is based on tapering the aperture and the other is based on modifying the feed.

\subsection{General Background}

There are several approaches for reducing the SLL of HMSIW and SIW LWAs. Tapering the via fence, the side aperture, slots sizes, and modifying the feed section are the most effective methods for the SLL reduction of SIW and HMSIW based LWAs. Two new approaches for reducing SLL of HMSIW and SIW LWAs are discussed in this chapter. The first approach is based on tapering the aperture for reducing the SLL of an HMSIW based LWA and discussed in Section 3.2. The second method is proposed in Section 3.3 for the SLL reduction of a SIW based LWA by modifying the feed transition. The design procedure, parametric study, simulated, and measured results are discussed thoroughly. A comparison with the state-of-the-art antennas is also made.

\subsection{Reduction of SLL by Tapering the Side Aperture}

One of the issues of the HMSIW LWA is the large SLL of the radiation pattern [63]. Tapering the side via fence is a convenient technique to reduce the SLL of the HMSIW LWA [64]-[66]. A new method for reducing the SLL of HMSIW LWA is proposed in this section. The proposed method is based on tapering the side aperture of the HMSIW LWA [69], [70]. 
The width of the HMSIW was chosen to enable single-mode propagation over the desired frequency bandwidth. Assuming the propagation of only the fundamental mode $\left(\mathrm{TE}_{0.5,0}\right)$, the width of the antenna was calculated by

$$
w=\frac{c}{4 \sqrt{\varepsilon_{r}} f_{c}}
$$

where $w, f_{c}, \varepsilon_{r}$, and $c$ are the width of the antenna, cut-off frequency, dielectric constant, and velocity of light in free space, respectively [63], [76]. According to (3.1), assuming $f_{c}$ $=24 \mathrm{GHz}$ and $\varepsilon_{r}=2.2$, the corresponding $w$ is $2.1 \mathrm{~mm}$. In SIW and HMSIW, the via fence acts as waveguide sidewall when (2.5) is satisfied, and $s / d$ and $d / w$ be smaller than 3 and 0.2 , respectively [35], [36]. The radiation length of the antenna was obtained by (2.4). $\Delta \theta$ and $\theta_{0}$ were selected as $15^{\circ}$ and $70^{\circ}$, respectively, resulting in $L_{r}=39 \mathrm{~mm}$ based on (2.4). The total length of the antenna depends on the feed length, which was optimized to achieve the minimum return loss and residual power. Therefore, the total length of the antenna is $70 \mathrm{~mm}$. It should be noted that $\theta_{0}$ was measured from the endfire (i.e., $\theta_{0}=0^{\circ}$ is at endfire). According to (2.3), changing $\beta$ leads to variations in $\theta_{0}$ and beam-scanning.

Since HMSIW is a compact dielectric-filled rectangular waveguide and the open aperture in the HMSIW acts as a magnetic wall, the same procedure as slotted rectangular waveguide was followed to find the radiation pattern. Assuming dominant mode $\left(\mathrm{TE}_{0.5,0}\right)$ propagation in the $z$-direction, the aperture electric field components were determined by

$$
\begin{gathered}
E_{y}=A_{m} k_{x} \sin k_{x}(w-x) \cos \left(k_{y} y\right) e^{-j k_{z} z} \\
E_{x}=A_{m} k_{y} \cos k_{x}(w-x) \sin \left(k_{y} y\right) e^{-j k_{z} z} \\
k_{x}=\frac{\pi}{2(w-a)}
\end{gathered}
$$




$$
\begin{gathered}
k_{z}=\sqrt{k_{0}^{2} \varepsilon_{r}-\frac{\pi^{2}}{4(w-a)^{2}}-\left(\frac{\pi}{h}\right)^{2}} \\
k_{y}=\frac{\pi}{h}
\end{gathered}
$$

where $A_{m}, h, a, k_{x}, k_{y}, k_{z}, E_{x}$, and $E_{y}$ are amplitude coefficient, the thickness of the board, location of the maximum electric field along the $\mathrm{x}$-axis, the $x, y$, and $z$ components of the wavenumber, $x$ and $y$ components of the aperture electric field, respectively [138]. The electric field distributions of the conventional (i.e., $W_{a}=0$ ) and tapered aperture (i.e., $W_{a}=0.13 \mathrm{~mm}$ ) HMSIW LWAs are presented in Fig. 3.1. According to Fig. 3.1, tapering the aperture shifted the locations of the maximum electric field (i.e., $a$ ), which changed $k_{x}, k_{z}$, and the electric field distribution according to (3.2)-(3.6). The far-zone fields are related to the aperture fields and surface currents through the fast Fourier transform (FFT) [138]. As a result, tapering the aperture disturbs the radiation pattern.

\subsubsection{Structure of the HMSIW LWA}

The proposed design was based on Rogers RT/Duroid 5880 substrate with 0.5 oz copper cladding, as presented in Fig. 3.2. The length, width, and thickness of the antenna are $70 \mathrm{~mm}, 11.5 \mathrm{~mm}$, and $0.127 \mathrm{~mm}$, respectively. The small size of the antenna makes it a suitable candidate for miniaturized mm-wave applications. The length and width of the antenna were determined following the procedure mentioned in sections 2.1 and 3.2. It should be noted that $w$ is the waveguide width of HMSIW, while the total width was chosen to minimize the radiation underneath the antenna. In other words, the extended copper cladding (beyond the via fence) acts as a ground plane/reflector. To achieve a low-profile antenna, the thickness of the antenna was chosen as $0.127 \mathrm{~mm}$ that is the 


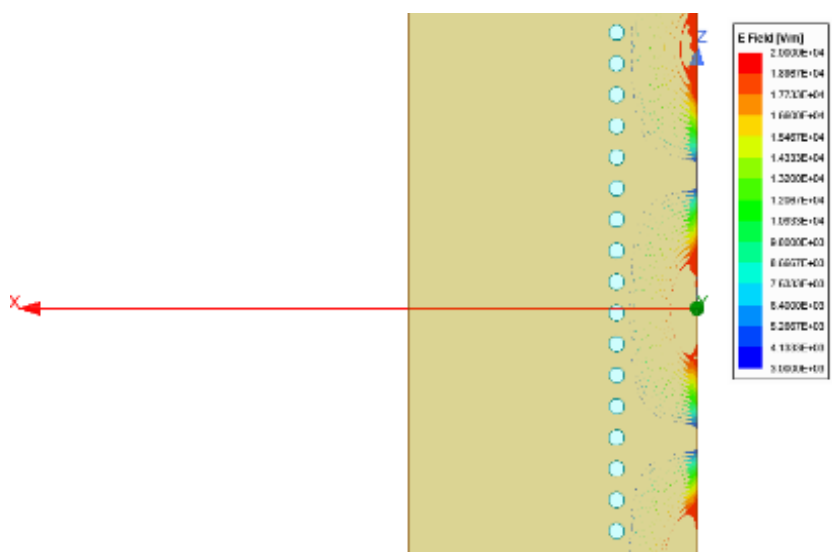

(a)

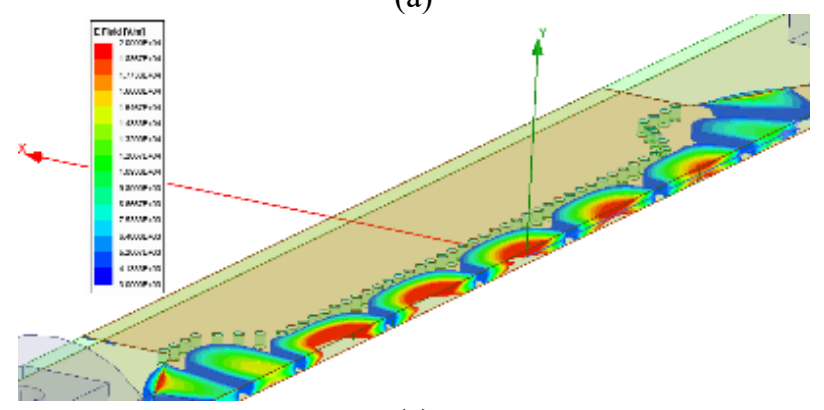

(c)

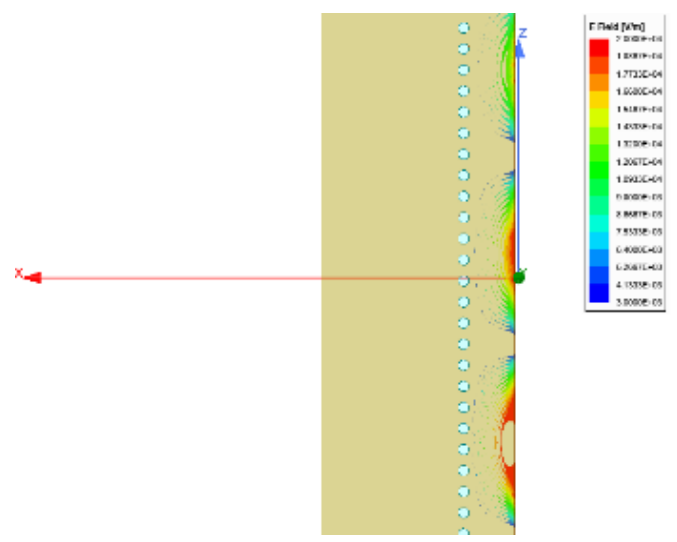

(b)

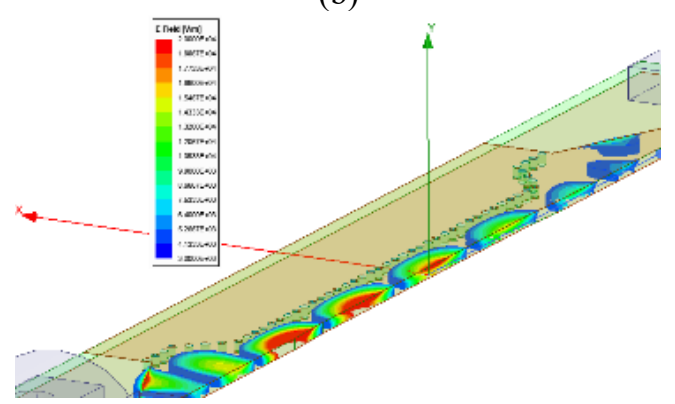

(d)

Figure 3.1. Electric field distributions of the conventional and tapered aperture HMSIW LWAs [70].

(a) Top view of E-field distribution in conventional HMSIW LWA (i.e., Wa = 0), (b) Top view of Efield distribution in tapered aperture HMSIW LWA (i.e., Wa $=0.13 \mathrm{~mm}$ ), (c) 3D view of E-field distribution in conventional antenna, and (d) 3D view of E-field distribution in HMSIW LWA with tapered aperture.

thinnest available substrate. However, similar to the waveguide structures, the small thickness of the substrate results in higher conductor loss and lower gain. Moreover, a thin substrate is more sensitive to mechanical stress. The mechanical strength of the antenna can be enhanced by adding an aluminum sheet as the ground plane.

In addition to the tapered microstrip transition, tapered side via fence was used to improve the impedance matching. Each end of the antenna was terminated with a $2.4 \mathrm{~mm}$ connector. One connector was terminated with a $50 \Omega$ matched load, while the other was connected to the signal generator. It should be noted that tapering the side aperture 
changes the impedance matching as well. To reduce the effect on the impedance matching, only part of the aperture corresponding to the radiation section was tapered.

Several tapering topologies for the side aperture were implemented, as demonstrated in Fig. 3.3. The chosen trapezoid configuration results in minimum SLL as compared to the other shapes. The ease of realization of the selected topology is another key benefit of the proposed configuration.

\subsubsection{Simulation Results}

The full-wave simulations have been carried out using Ansys HFSS v.18. The Sparameters results are presented in Fig. 3.4. According to Fig. 3.4, the proposed antenna exhibits a return loss better than $10 \mathrm{~dB}$ over the $2.6 \mathrm{GHz}$ bandwidth ranging from 26.6 $\mathrm{GHz}$ to $29.2 \mathrm{GHz}$. The corresponding insertion loss is ranging from $6 \mathrm{~dB}$ to $15 \mathrm{~dB}$ indicating the small residual un-radiated power.

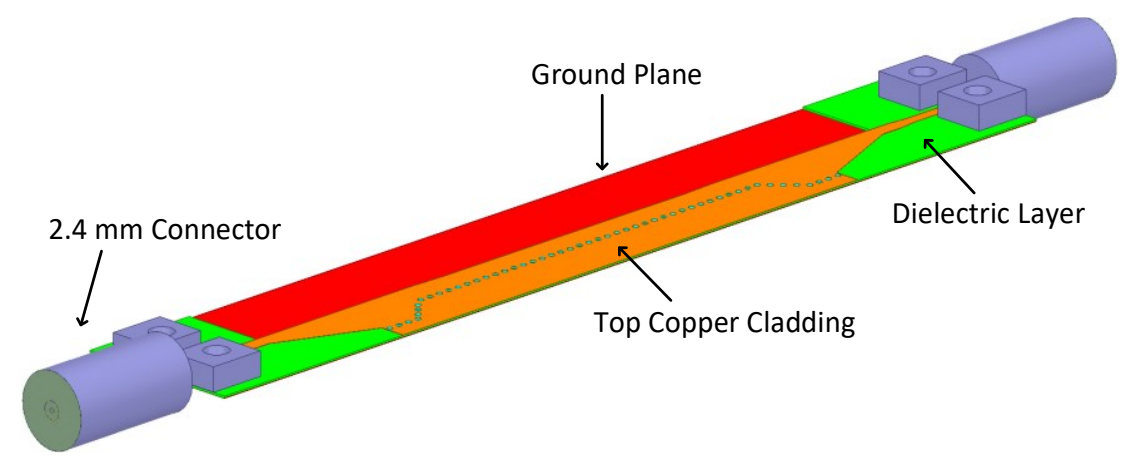

(a)

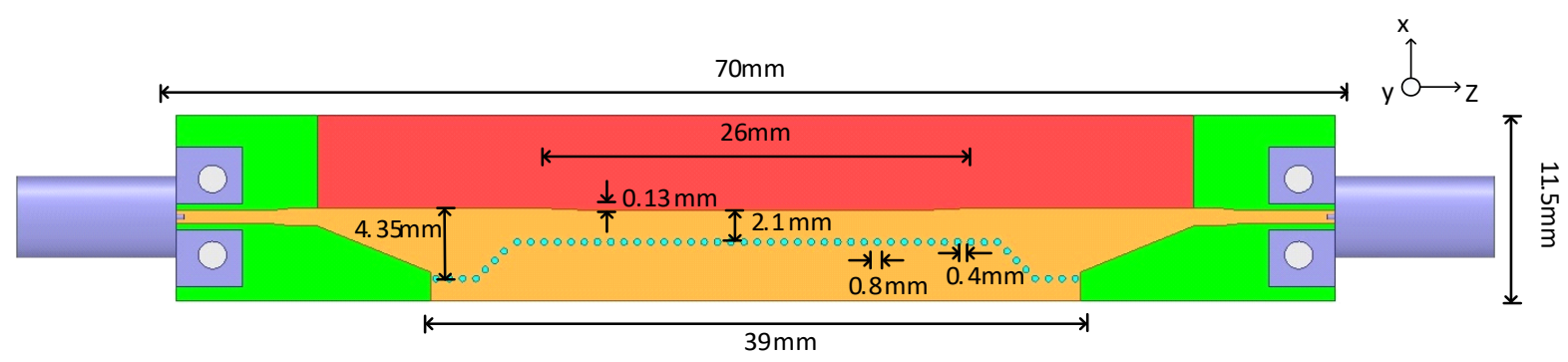

(b)

Figure 3.2. Schematic of the proposed HMSIW LWA with reduced SLL. (a) 3D view, (b) top view [69]. 


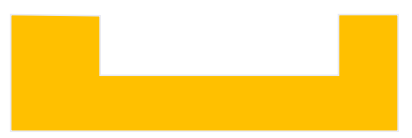

(a)

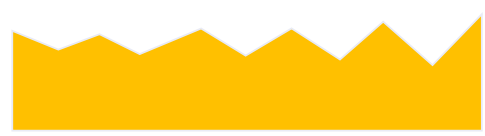

(b)

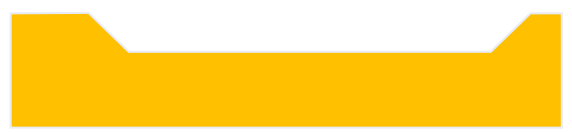

(c)

Figure 3.3. Different topologies for tapering the side aperture. (a) rectangular shape, (b) triangular/saw shape, (c) trapezoid shape.

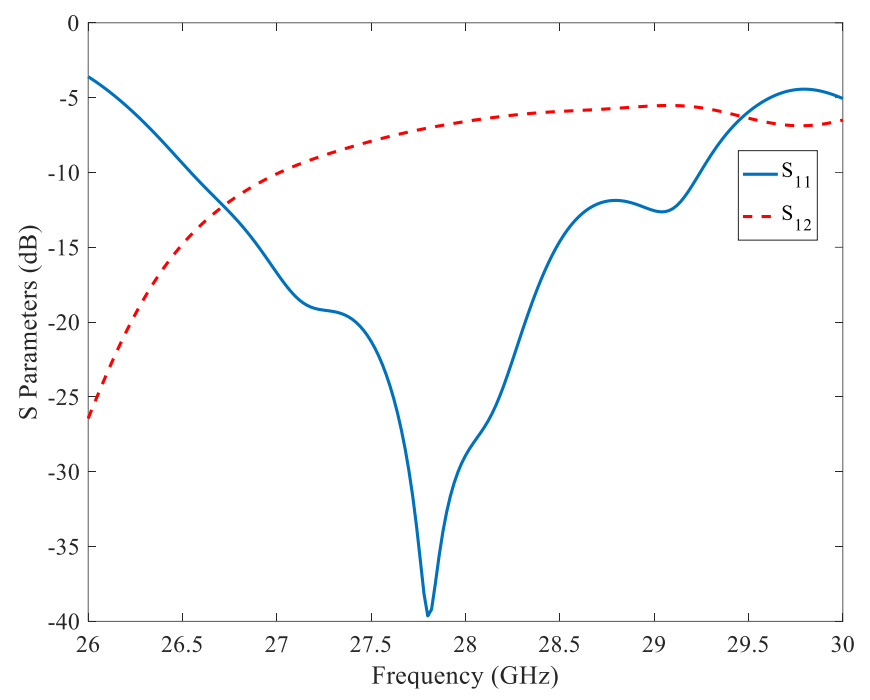

Figure 3.4. Simulated S-parameters of the proposed $0.127 \mathrm{~mm}$ HMSIW LWA with reduced SLL [69].

The radiation gain patterns of the antenna at different frequencies are presented in Fig. 3.5 , indicating that the main-lobe of the radiation pattern rotates and the beam scans space by sweeping the frequency. The frequency sensitivity of the radiation pattern was reduced by tapering the aperture, which led to the reduction of the effective dielectric constant. This is advantageous in applications where large frequency beam-squint is not desirable. According to Fig. 3.5, the proposed antenna scans space with medium gain and low SLL. The simulated realized gain, SLL, and main-lobe angle of the antenna in the Eplane (YZ-plane) at $28.5 \mathrm{GHz}$ (center frequency) corresponding to about $10 \mathrm{dBi}$, $-19 \mathrm{~dB}$, and $55^{\circ}$, respectively [69]. The SLL cannot be reduced beyond some point while obtaining proper gain and low return loss. This is because of the trade-off between return loss, SLL, and gain. 


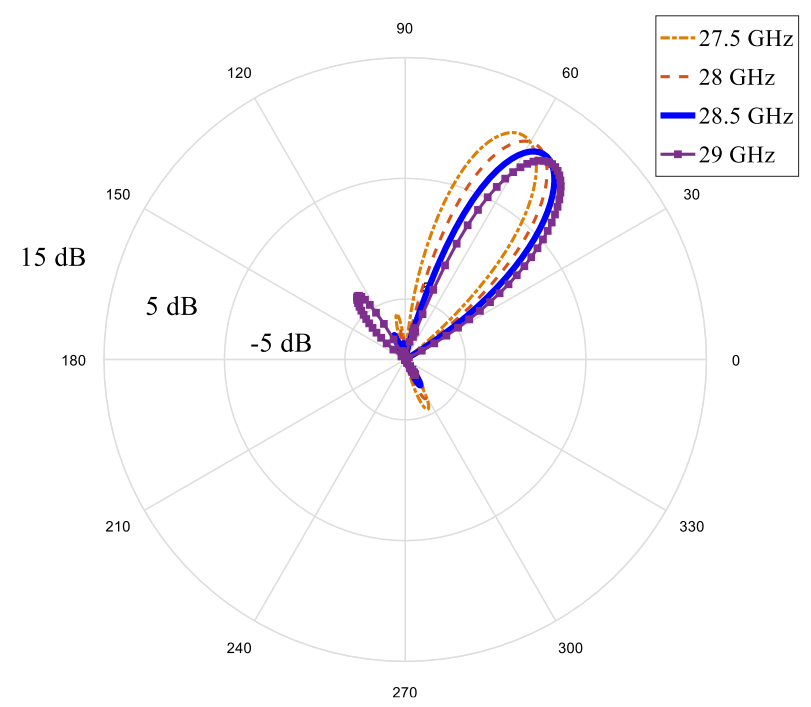

Figure 3.5. Simulated radiation patterns of the proposed thin HMSIW LWA at E-plane (YZ-plane)

$$
\left(\varphi=90^{\circ}\right)[69] .
$$

To increase the mechanical strength of the antenna and make the fabrication easier, the proposed method was realized on a thicker $(0.5 \mathrm{~mm})$ substrate. The simulated and measured results of the proposed antenna are discussed in Section 3.2.3.

\subsubsection{Fabrication and Measured Results}

Due to the PCB fabrication technology limitation and softness of the PTFE material, a precise tapered aperture is not achievable in thin substrates such as $0.127 \mathrm{~mm}$ substrate. Hence, the proposed design procedure was realized on a thicker Roger RT/Duroid 5880 substrate with the $\tan (\delta)=0.0009$. The small dielectric loss was a key factor for selecting this substrate as the backbone of the proposed antenna. Following the design procedure, the length, width, and thickness of the fabricated antenna are $70 \mathrm{~mm}, 15 \mathrm{~mm}$, and 0.5 $\mathrm{mm}$, respectively. The fabricated antenna is presented in Fig. 3.6. Some of the important geometrical parameters of the antenna are reported in Table 3.1. Both ends of the antenna were terminated with k-connectors, while the output was connected to a $50 \Omega$ matched load to absorb the non-radiated power. Since the impedance of the radiating section of the 
antenna is different from the input impedance, a tapered via fence was implemented to improve the impedance matching. The surface mounted connectors were connected to the ground through screws (shown as grey cylinders) on the sides of the center pin, acting as a co-planar waveguide (CPW), which justifies the use of CPW to microstrip line transitions at each end. The vias were filled with a conductive resin (e.g., silver paste) to replicate the sidewall of the waveguide and confine the fields of $\mathrm{TE}_{\mathrm{n} 0}$ modes. The side aperture of the antenna was tapered in a trapezoid shape with $W_{a}$ width to reduce SLL. An exaggerated view of the side aperture is shown in Fig. 3.6(b) to highlight the tapered side aperture. Due to fabrication technology limitations, it was impossible to taper the dielectric layer and top copper cladding without cutting the ground plane. Hence, the top cladding, the dielectric layer, and the ground plane were cut similarly.

The S-parameters were measured at Carleton University using a Rohde \& Schwarz ZVA-67 VNA. The S-parameter measurement setup is shown in Fig. 3.7. The measured and simulated S-parameters are presented in Fig. 3.8. According to Fig. 3.8, the proposed antenna exhibits a return loss better than $10 \mathrm{~dB}$ over the frequency band of $26 \mathrm{GHz}$ to 30 GHz. The insertion loss ranges from $8 \mathrm{~dB}$ to $12 \mathrm{~dB}$ over the $10 \mathrm{~dB}$ impedance bandwidth (i.e., $26-30 \mathrm{GHz}$ ). This shows that about $10 \%$ of the input power reaches the matched load at the output, which confirms the reduction of SLL by tapering the side aperture.

Table 3.1. Some of the important geometrical parameters of the proposed LWA [70].

\begin{tabular}{|c|c|c|c|c|}
\hline Parameter & $h$ & $W$ & $W_{t}$ & $W_{a}$ \\
\hline Value (mm) & 0.5 & 2.1 & 15 & 0.13 \\
\hline
\end{tabular}




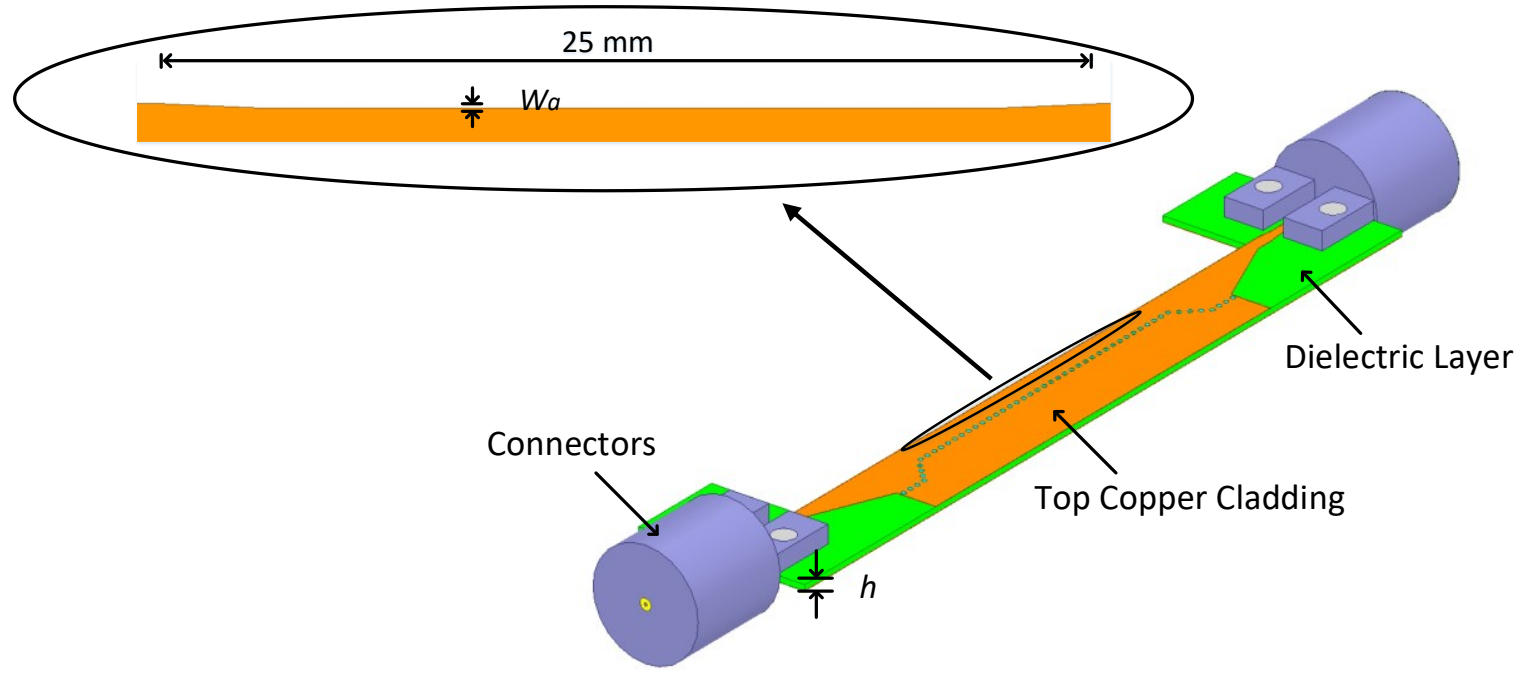

(a)

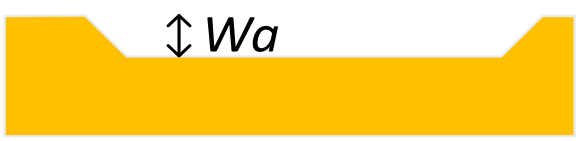

(b)

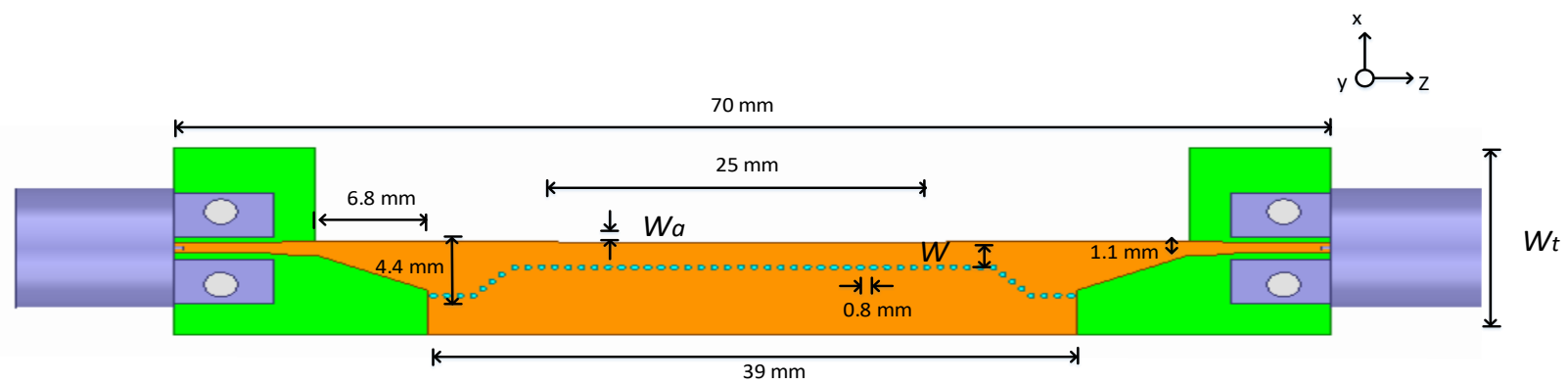

(c)

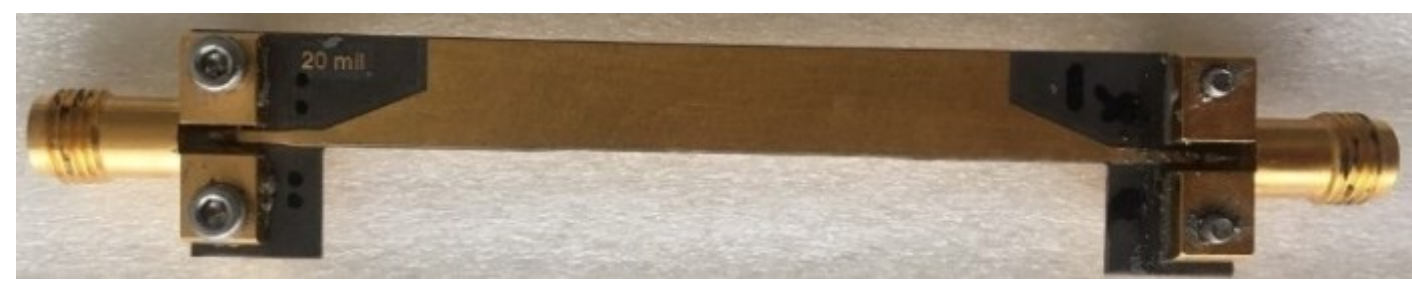

(d)

Figure 3.6. Schematic view of the fabricated HMSIW LWA. (a) 3D view (tapered side aperture of the width of $W_{a}$ was circled in the same view), (b) the exaggerated non-scaled view of the tapered aperture, (c) top view, and (d) manufactured antenna [70]. 


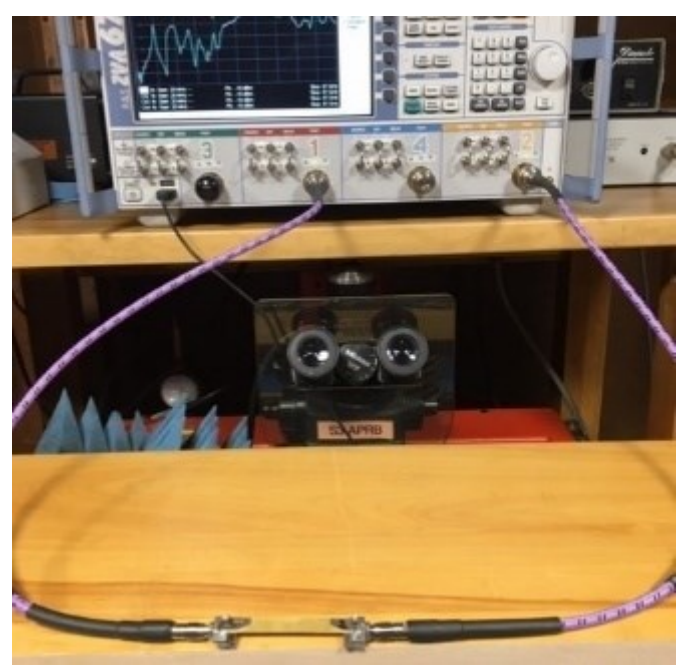

Figure 3.7. S-parameters measurement setup of the proposed HMSIW LWA [70].

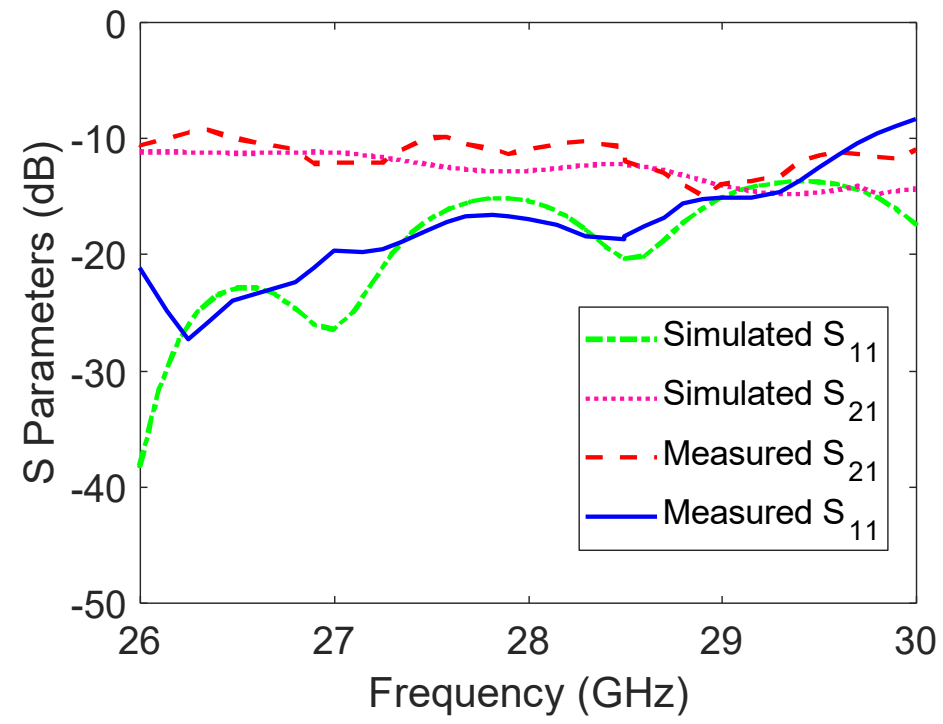

Figure 3.8. S-parameters of the fabricated HMSIW LWA with reduced SLL.

The radiation gain patterns of the proposed HMSIW LWA were measured at the PolyGram anechoic chamber in Polytechnique De Montreal. The measurement setup is presented in Fig. 3.9. A standard gain horn antenna MI-12A-26 with an average peak gain of $23.6 \mathrm{dBi}$ over the frequency band of $26.5 \mathrm{GHz}$ to $30 \mathrm{GHz}$ was used. It should be noted that the reported radiation patterns in this dissertation correspond to the E-plane (YZ-plane). Unfortunately, measuring the H-plane is out of the capability of the measurement system. This is due to the fact that the antenna's main-beam is tilted from 
the broadside and the antenna needs to be steered to measure the H-plane. Moreover, the reported radiation patterns are the realized gains, which take the mismatch into account.

The simulated and measured radiation patterns of the proposed HMSIW LWA at 28.5 $\mathrm{GHz}$ are presented in Fig. 3.10, confirming that tapering the aperture leads to SLL improvement [70]. It should be noted that the conventional HMSIW LWA resembles $W_{a}=0$, while the tapered aperture HMSIW LWA corresponds to $W_{a}=0.13 \mathrm{~mm}$. The radiation patterns of the proposed antenna at different frequencies are shown in Fig. 3.11, indicating frequency beam-scanning with relatively low SLL.

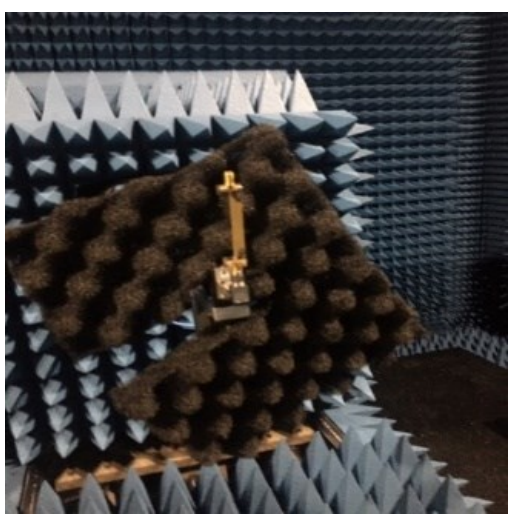

(a)

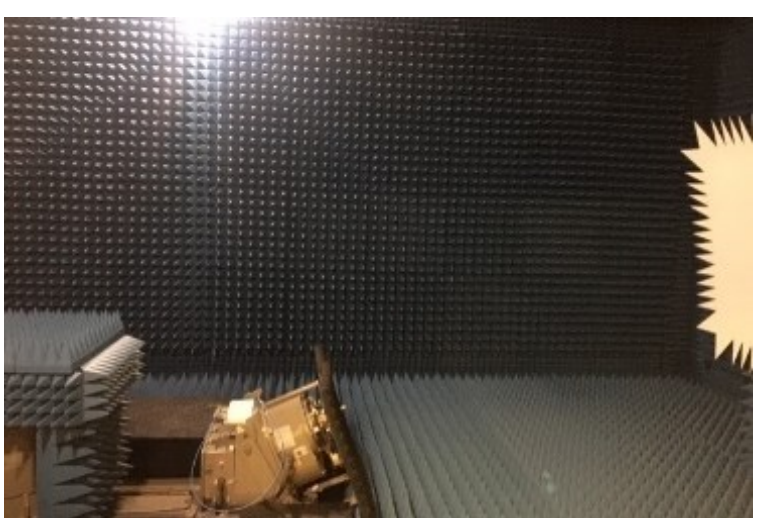

(b)

Figure 3.9. Measurement setup for measuring the radiation pattern of the proposed HMSIW LWA.

(a) antenna under test (AUT), (b) anechoic chamber [70].

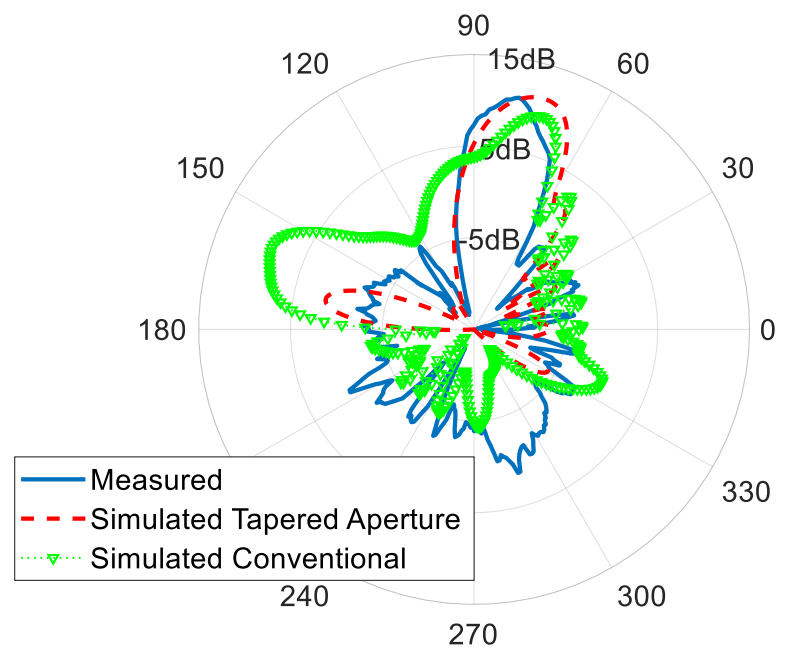

Figure 3.10. Radiation patterns of the fabricated HMSIW LWA at $28.5 \mathrm{GHz}$ and E-plane [70]. 

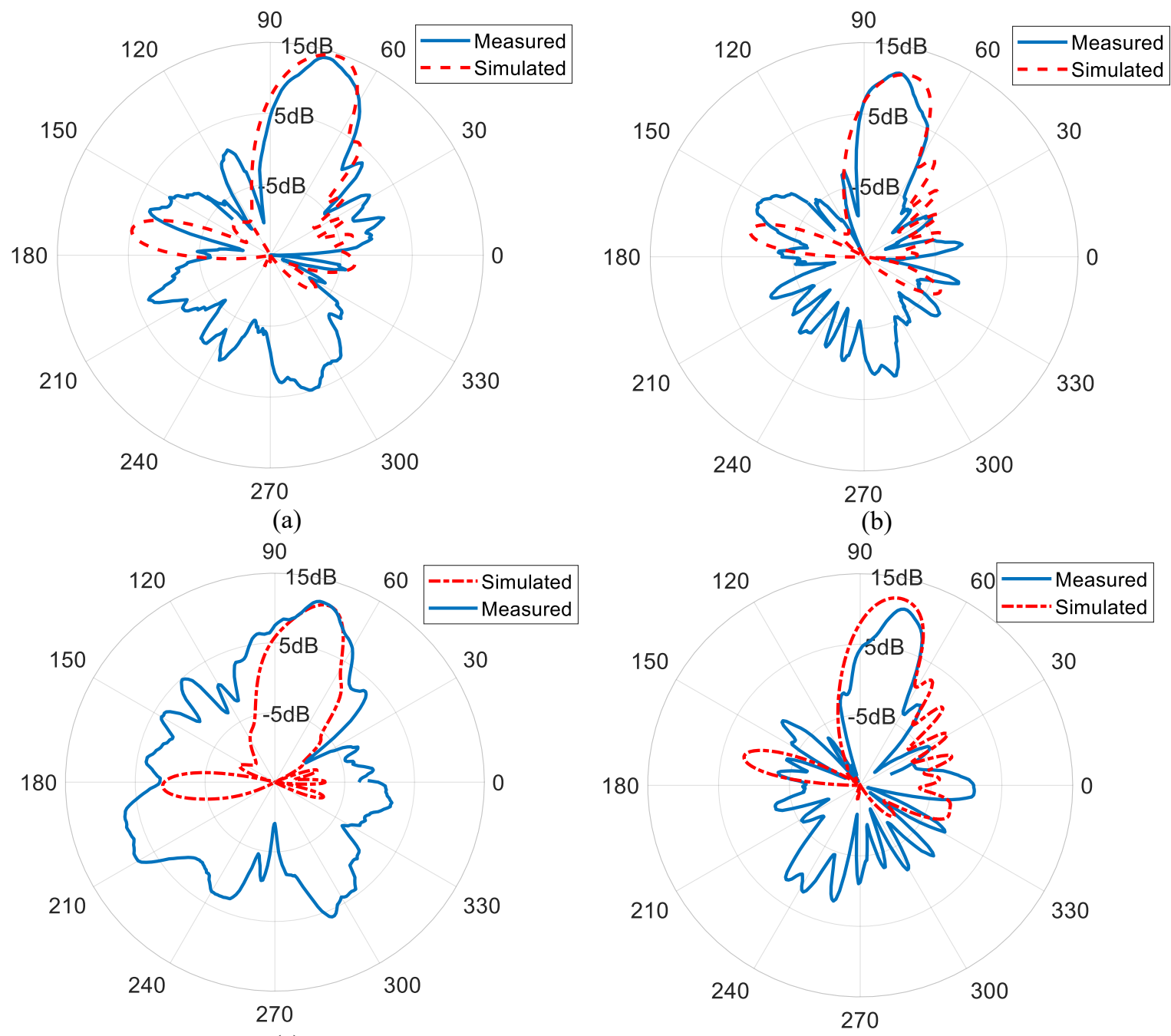

(c)

(d)

Figure 3.11. Radiation patterns of the proposed HMSIW LWA at E-plane $\left(\varphi=90^{\circ}\right)$ and different frequencies [70]. (a) at $28 \mathrm{GHz}$, (b) at $29 \mathrm{GHz}$, (c) $26.5 \mathrm{GHz}$, and (d) at $29.5 \mathrm{GHz}$.

As stated before, HMSIW has two main-lobes in E-plane [63]. The absence of a large ground plane causes the appearance of a second main-beam. Reflection from the feed transition and the aperture can also lead to the existence of the second main-beam. Tapering the side aperture results in the SLL of $-13.8 \mathrm{~dB}$ and $-11.2 \mathrm{~dB}$ in the upper hemisphere and full space, respectively. The SLL changes by sweeping the frequency due to the leaky-wave nature of the proposed antenna [28]-[30]. Truncating the dielectric layer reduced the effective dielectric constant closer to one, leading to the reduction of 
the frequency sensitivity. In other words, the beam scanned space at a slower rate. This is beneficial for applications where large frequency beam-squint is not desirable. The simulated and the measured peak realized gain of the proposed antenna are shown in Fig. 3.12 , indicating the medium to high gain in the frequency band of $26-30 \mathrm{GHz}$. It should be noted that the peak realized gain is more realistic than the peak gain since the Sparameters were considered in it.

To establish the advantages of the proposed design, a comparison is made in Table 3.2 with similar reported antennas. Our proposed antenna exhibits the lowest SLL in full space compared to [63]-[68], confirming the key advantage of our proposed design. SLL of our proposed LWA in the upper hemisphere is lower than the reported ones in [63], [64], [66], and [67]. The reported SLL at the upper hemisphere in [65] and [68] is lower, but the radiation patterns in [65] and [68] were only considered in the upper hemisphere. Our reported peak realized gain is lower than the reported ones in [63], [65], and [68] which is due to the longer length of those antennas. Our operating frequency bandwidth is similar to the one reported in [63], while the length of the antenna in [63] is almost twice our proposed LWA. This shows that the antenna can be miniaturized while gain remains almost constant. Overall, Table 3.2 indicates that the proposed structure is a suitable candidate for $5 \mathrm{G} \mathrm{mm}$-wave point to point communication. 


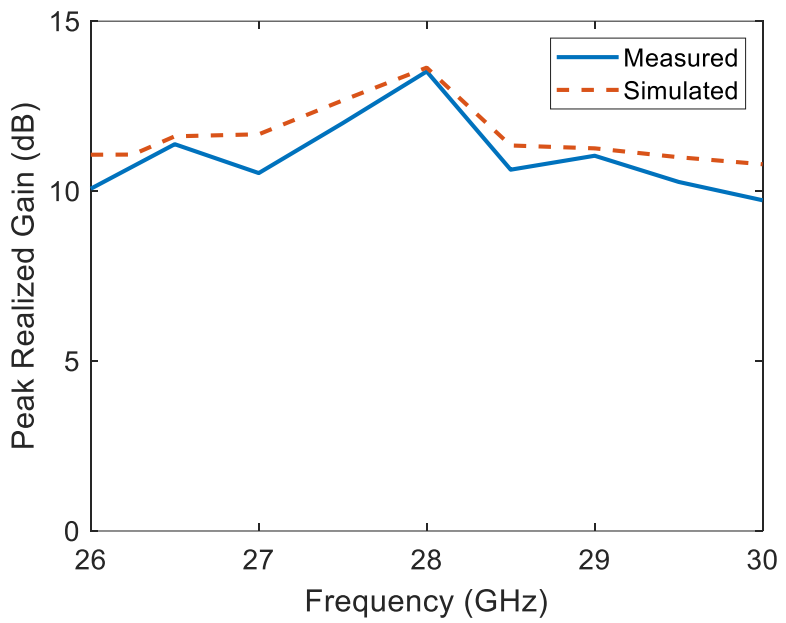

Figure 3.12. Peak realized gain of the proposed HMSIW LWA [70].

Table 3.2. Comparison among similar antennas in terms of SLL, peak gain, frequency band, and antenna length [70].

\begin{tabular}{|c|c|c|c|c|c|}
\hline Design & $\begin{array}{c}\text { SLL upper } \\
\text { hemisphere }\end{array}$ & SLL full-space & Peak Gain & $\begin{array}{c}\text { Frequency } \\
\text { Band }\end{array}$ & $\begin{array}{c}\text { Antenna } \\
\text { Length }\end{array}$ \\
\hline $\begin{array}{c}\text { Our proposed } \\
\text { HMSIW LWA } \\
\text { with tapered } \\
\text { aperture }\end{array}$ & $-13.8 \mathrm{~dB}$ & $-11.2 \mathrm{~dB}$ & $10.6 \mathrm{dBi}$ & $26-30 \mathrm{GHz}$ & $6 \times \lambda$ \\
\hline$[63]$ & $-12.5 \mathrm{~dB}$ & $-1 \mathrm{~dB}$ & $11.7 \mathrm{dBi}$ & $25-28 \mathrm{GHz}$ & $12 \times \lambda$ \\
\hline$[65]$ & $-23.8 \mathrm{~dB}$ & Not reported & $15.6 \mathrm{dBi}$ & $6.5-10 \mathrm{GHz}$ & $8 \times \lambda$ \\
\hline$[64]$ & $-4 \mathrm{~dB}$ & $-1 \mathrm{~dB}$ & $6 \mathrm{dBi}$ & $6.7-17 \mathrm{GHz}$ & $10 \times \lambda$ \\
\hline$[67]$ & $-12 \mathrm{~dB}$ & $-10.5 \mathrm{~dB}$ & $5.8 \mathrm{dBi}$ & $2.98-3.04 \mathrm{GHz}$ & $1.5 \times \lambda$ \\
\hline$[66]$ & $-13 \mathrm{~dB}$ & Not Reported & $10 \mathrm{dBi}$ & $8.5 \mathrm{GHz}$ & $4 \times \lambda$ \\
\hline$[68]$ & $-15 \mathrm{~dB}$ & $-9 \mathrm{~dB}$ & $18.2 \mathrm{dBi}$ & $5.78-5.82 \mathrm{GHz}$ & $7.8 \times \lambda$ \\
\hline
\end{tabular}

Due to the small thickness of the Rogers RT/Duroid 5880 board, the antenna becomes more vulnerable to mechanical stress. Difficulty in achieving a clean cut, due to the softness of the PTFE material, is another factor causing discrepancies between measured and simulated results. Fabrication errors such as air gaps in the vias lead to undesired leakage through the via fence. 
A sensitivity analysis was carried out to investigate discrepancies between simulated and measured results more thoroughly. Therefore, some of the important structural parameters such as the thickness of the substrate $(h)$, the width of the antenna $\left(W_{t}\right)$, waveguide section width $(W)$, and the aperture size $\left(W_{a}\right)$ were changed individually and their impacts on antenna performance were investigated subsequently through the simulations. It should be noted that in each case to study the effect of each parameter individually, only one parameter was changed while the others were held constant. The S-parameters for different combinations are reported in Fig. 3.13. Reducing $W$ increases

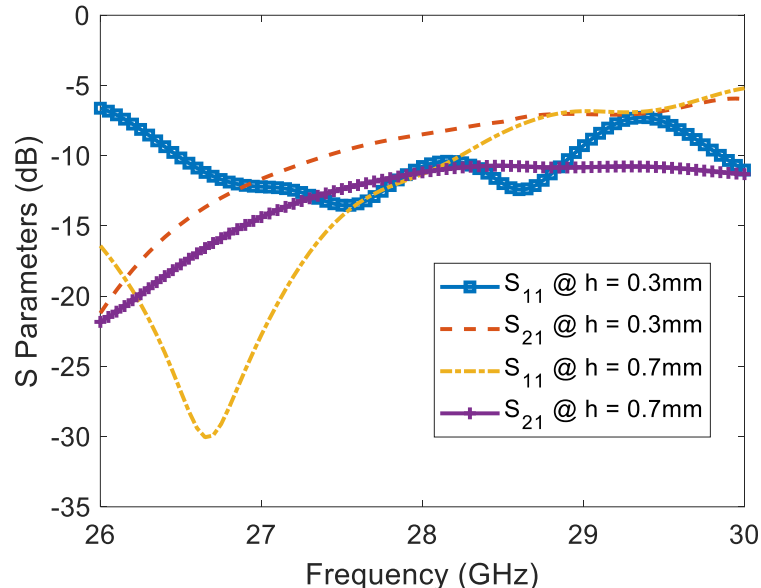

(a)

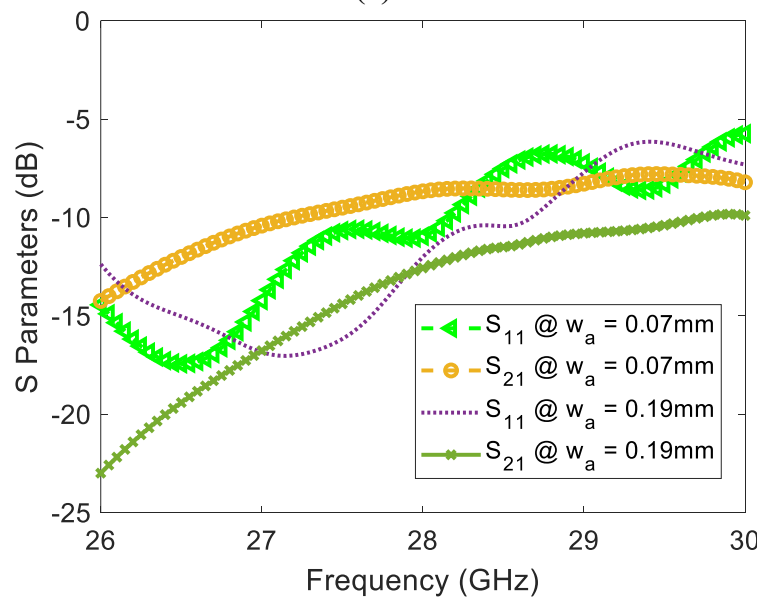

(c)

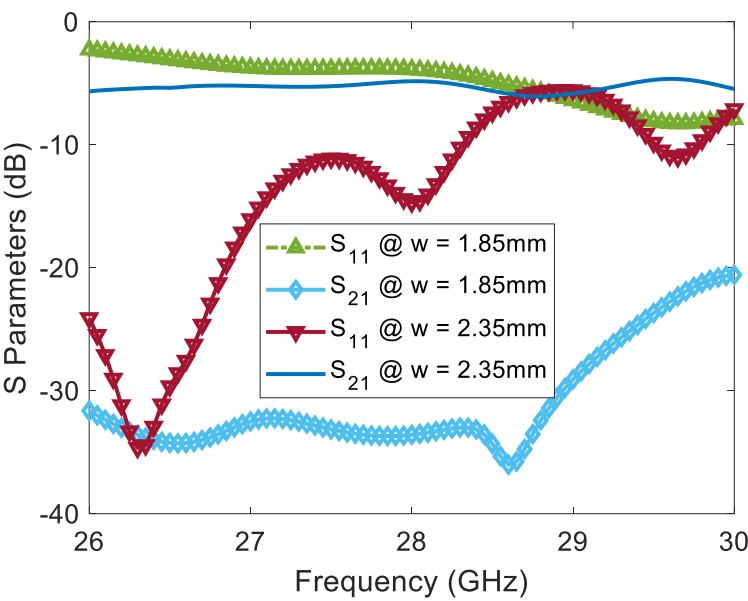

(b)

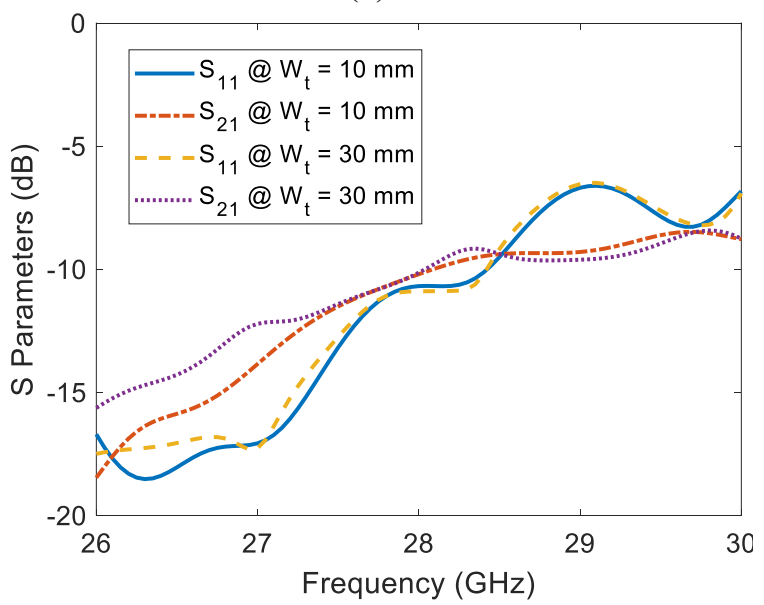

(d)

Figure 3.13. S-parameters of the proposed antenna for different combinations. (a) different values of $h$, (b) different values of $W$, (c) different values of $W_{a}$, and (d) different values of $W_{t}[70]$. 
the cutoff frequency $\left(f_{c}\right)$, affecting the propagation constant and modal structure. Hence, as presented in Fig. 3.13, return loss changes drastically by varying $W$. The normalized radiation patterns for different parameters are illustrated in Fig. 3.14. According to Fig. 3.14, $W_{a}$ and $W$ play the dominant roles in SLL. Hence, small fabrication errors in tapering the aperture and placing the via fence lead to the discrepancies. The peak realized gains of the antenna for different parameters are reported in Fig. 3.15. Overall, this parametric study indicates that $W$ contributes the most to the deviations in Sparameters and peak realized gain.

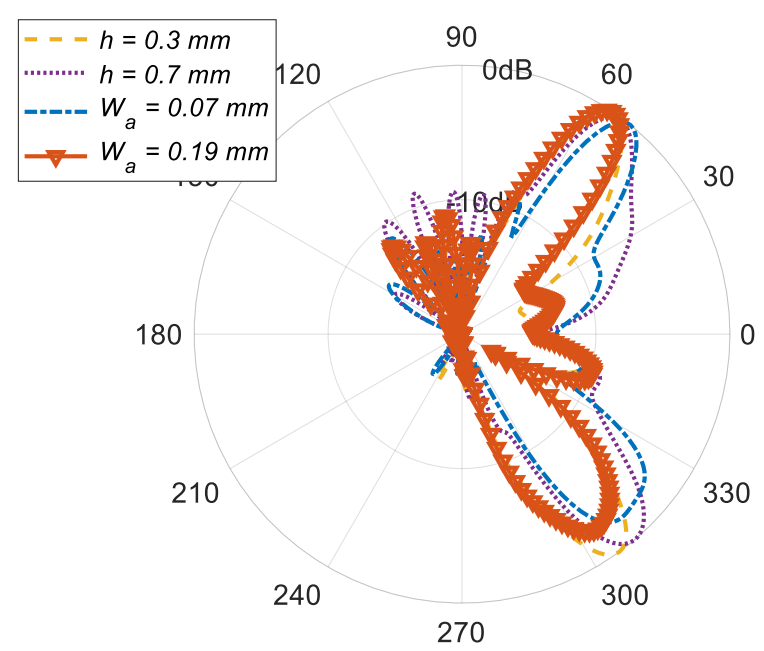

(a)

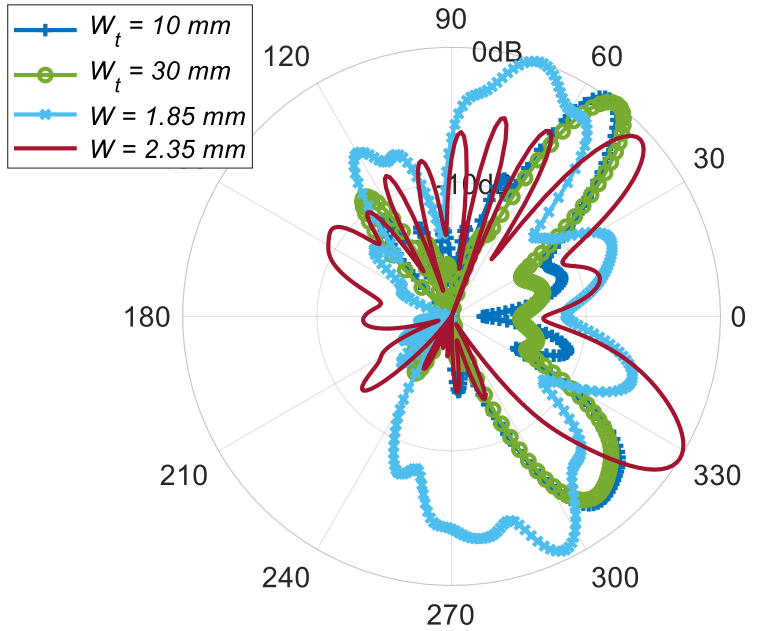

(b)

Figure 3.14. Normalized radiation patterns of the proposed HMSIW LWA obtained by changing different parameters at 28.5 GHz. (a) E-plane for different values of $h$ and $W_{a}$, (b) E-plane for different values of $W$ and $W_{t} \cdot[70]$ 


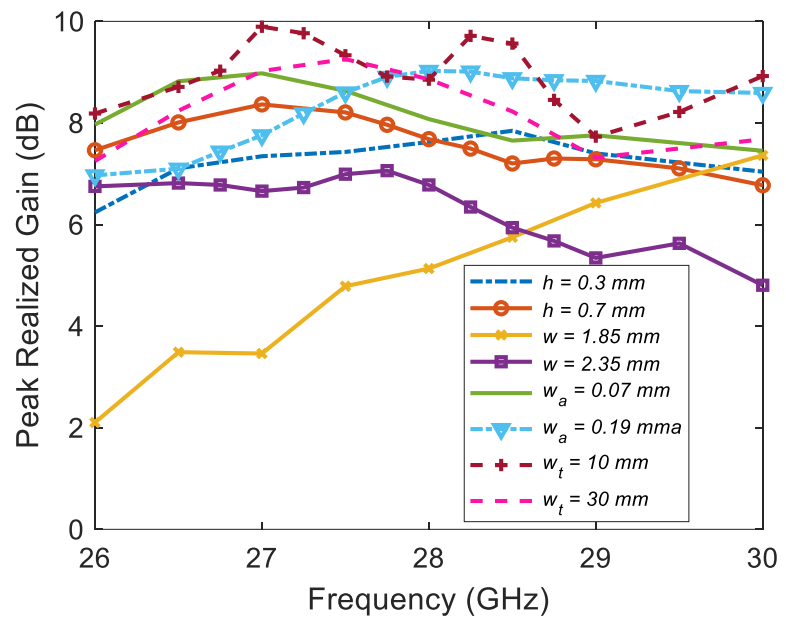

Figure 3.15. Peak realized gain of the proposed HMSIW LWA obtained by changing different parameters [70].

Attaching the connectors may bend the ends of the antenna because of the small thickness and relatively long length of the antenna. To simulate this situation, the connector was rotated to make an angle $\xi$ from the horizon (XZ plane), as shown in Fig. 3.16. The S-parameters, peak realized gain, and normalized radiation patterns at 28.5 GHz are reported in Figs. 3.17-3.19, respectively. According to Figs. 3.17 and 3.19, sweeping $\xi$ leads to higher SLL and smaller return loss. Since the connector center pin does not lie on the signal trace completely in this situation, a mismatch occurs, and most of the input power is reflected toward the signal generator. This degrades the antenna performance in terms of SLL and the return loss. According to Fig. 3.19, by sweeping $\xi$, the lobe level of the beams radiating underneath the antenna increase. This is the result of a large mismatch and unwanted reflections from the connectors.

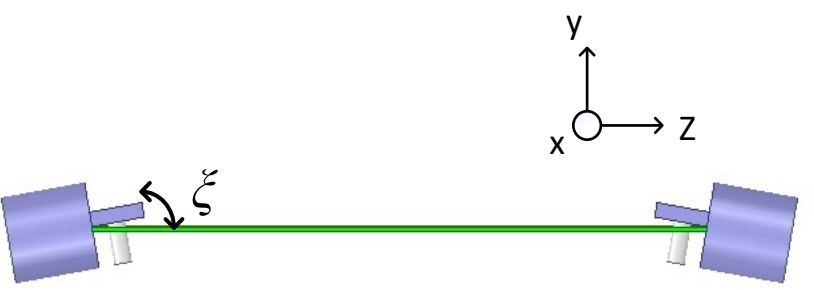

Figure 3.16. Schematic view of the proposed LWA with connectors offset from the horizon by $\xi$ [70]. 


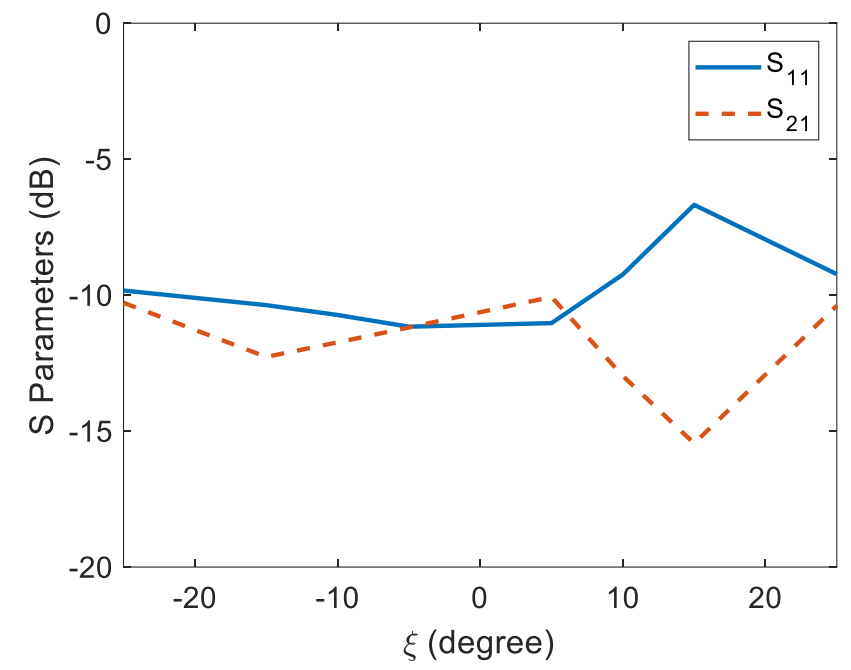

Figure 3.17. S-parameters of the proposed HMSIW LWA with rotated connectors at $28.5 \mathrm{GHz}[70]$.

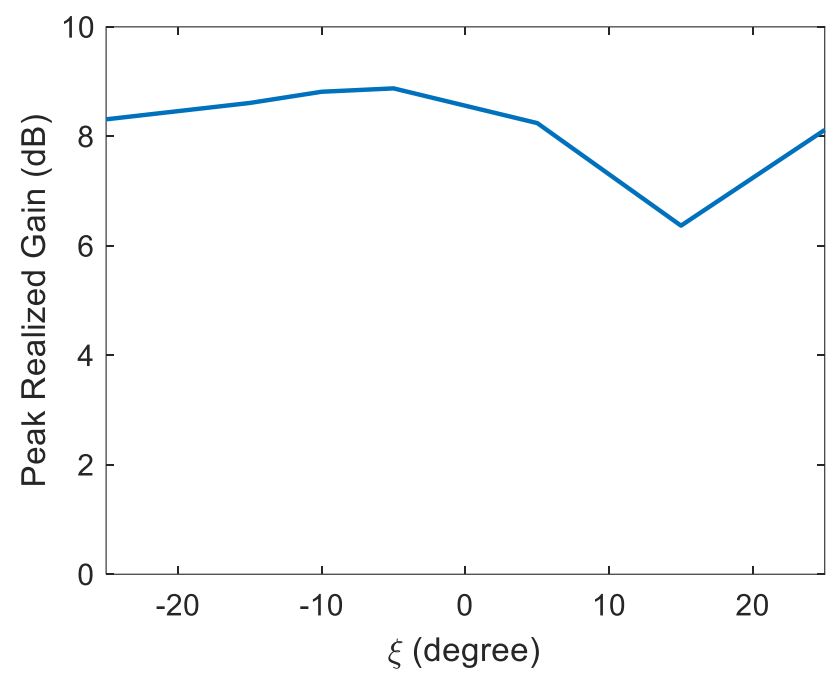

Figure 3.18. Peak realized gain of the proposed HMSIW LWA obtained with offset connectors at $28.5 \mathrm{GHz}[70]$. 


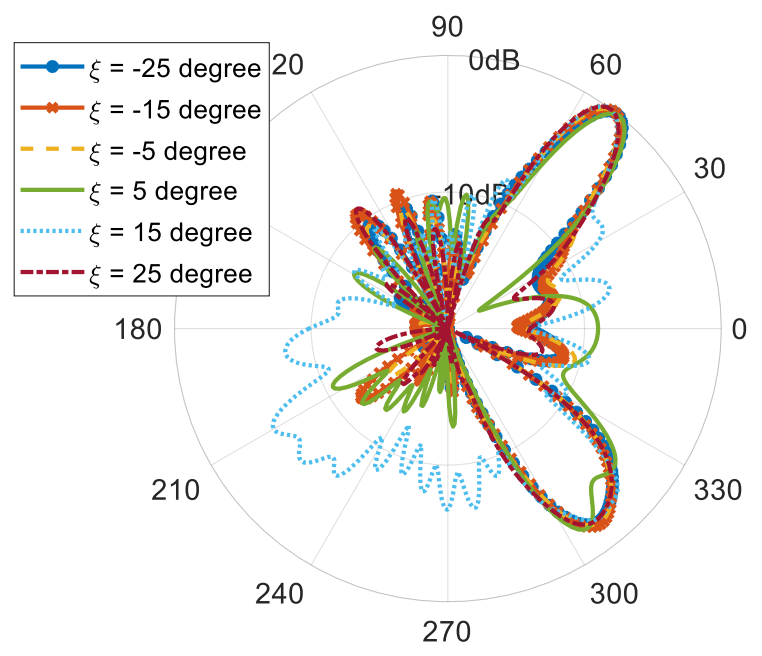

Figure 3.19. Normalized radiation patterns of the proposed HMSIW LWA with offset connectors at 28.5 GHz and E-plane $\left(\varphi=90^{\circ}\right)[70]$.

The antenna may also bend under the physical stress due to the small thickness of the board, as presented in Fig. 3.20. To simulate such a case, the antenna was bent with the gradual deviation from the horizon up to $h_{b}=6 \mathrm{~mm}$ by moving toward the two ends of the antenna. The S-parameters, normalized radiation patterns, and peak realized gain of the bent HMSIW LWA are reported in Figs. 3.21-3.23, respectively. According to Figs. 3.21-3.23, bending the antenna degrades the antenna response severely in terms of return loss, peak realized gain, and SLL. To avoid such case, dummy layers can be added underneath the ground plane. However, this increases the size of the antenna that is not suitable for miniaturized applications.

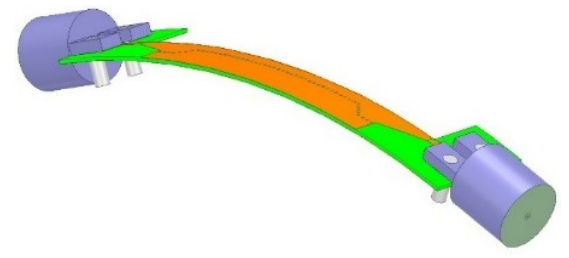

(a)

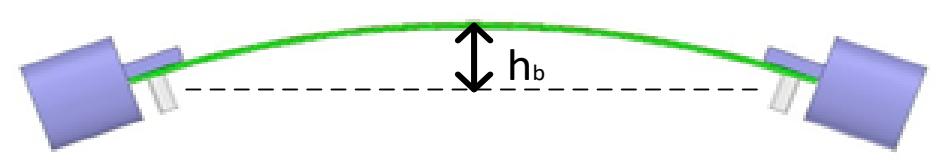

(b)

Figure 3.20. Schematic view of the bent antenna. (a) 3D view, (b) side view [70]. 


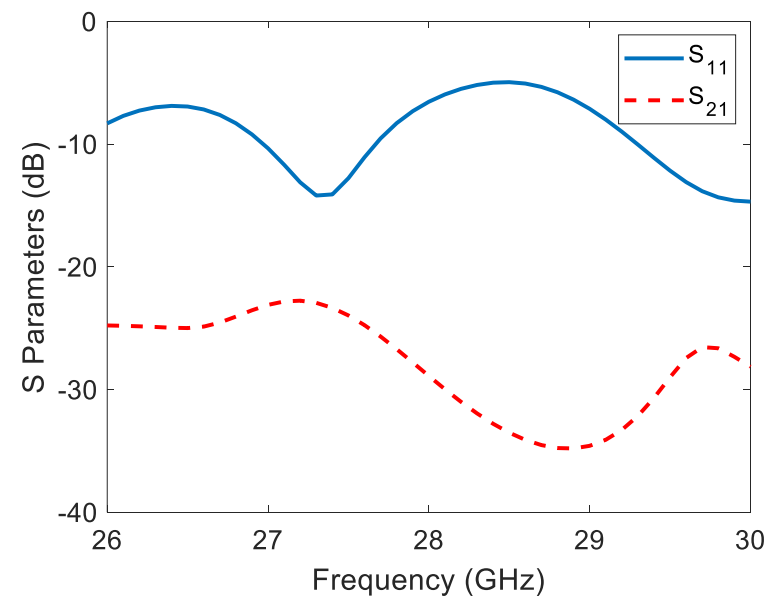

Figure 3.21. S-parameters of the proposed bent HMSIW LWA [70].

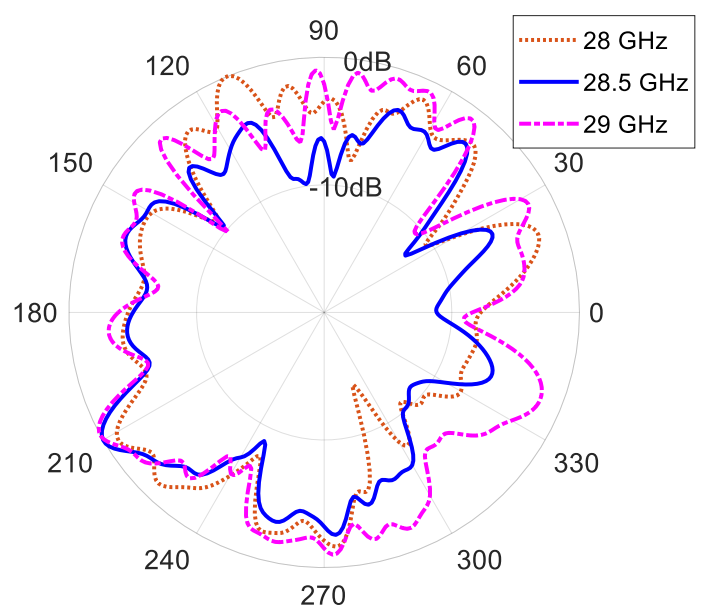

Figure 3.22. Normalized radiation patterns of the proposed bent HMSIW LWA [70].

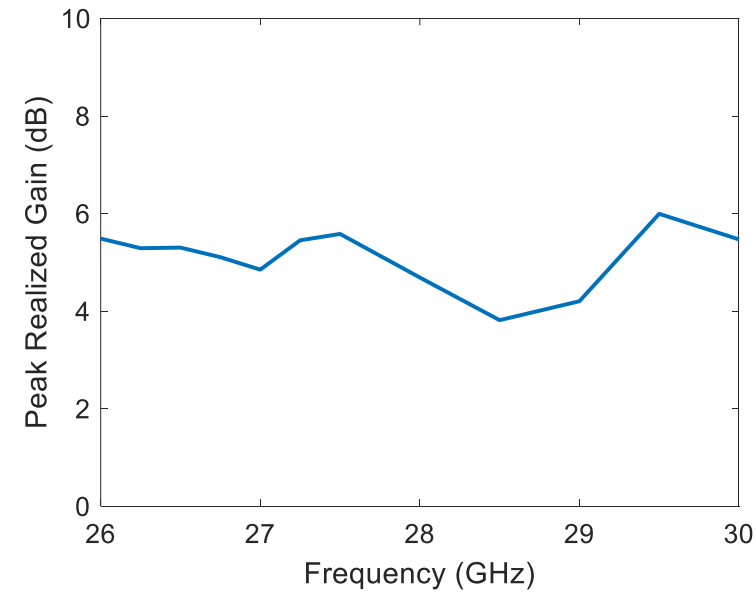

Figure 3.23. Peak realized gain of the proposed bent HMSIW LWA [70].

According to the parametric analysis, $W$ and $\xi$ contribute the most to the discrepancies between measured and simulated results. In other words, the discrepancies are mostly due 
to possible bending of the board, the errors in the assembly of connectors, and errors in placing the via fence. Furthermore, if the via fences were not filled completely with the conductive resin, the leaky-wave would not be confined, and discrepancies occur.

\subsection{Reduction of SLL by Modifying the Feed}

The planar antenna can be fed using surface-mounted or through-hole connectors. Surface-mounted connectors are more suitable for the integration and miniaturized applications due to their compactness. To connect the surface-mounted connectors to the SIW, tapered microstrip transition is often implemented. This is because implementing through-hole feed structures in the low-profile SIW antennas is a practical challenge. Hence, using a microstrip transition is a preferred feeding method for integration purposes. However, microstrip lines have unwanted radiation at high frequencies [142]. This unwanted radiation becomes worse by increasing the frequency or reducing the thickness [142]. The spurious effects result in the reduction of radiation efficiency because a large portion of the power leaks into space from the feed transition before reaching the radiation section of the antenna (e.g., slots). The undesired radiation from the feed results in a high SLL that can jeopardize signal detection in the face of interference. Overall, the undesired radiation from the microstrip transition at high frequencies is a significant challenge for mm-wave low-profile antennas.

In this section, the SLL of SIW LWA was reduced by modifying the feed transition. This is because modifying the feed can compensate for such undesired effects on the radiation pattern. The feed modification occurred by adding tapered CPW to each microstrip transition. This was achieved by placing two tapered conductive layers adjacent to each microstrip transition. It is similar to introducing additional parasitic 
capacitances to the structure and changing the electrical length of the antenna. The additional CPWs were tapered to minimize SLL.

\subsubsection{Structure of the SIW LWA}

The antenna was implemented on Rogers RT/Duroid 5880 substrate, as presented in Fig. 3.24. The radiating length of the antenna was obtained from (2.4), while the waveguide width was extracted from (2.5) and (2.6). As stated before, the total length of the antenna depends on the feed transition's length, which was optimized to achieve the adequate return loss. In SIW, similar to HMSIW, the copper claddings beyond the via fences (along $\mathrm{X}$-axis) act as ground plane and prevent radiation underneath the antenna. Hence, the total width was chosen to minimize the unwanted radiation underneath the antenna. As stated before in Section 3.2, thickness of the antenna affects the loss, SLL, and gain. Therefore, the thickness of the antenna was chosen as $1.3 \mathrm{~mm}$ to achieve a balance between gain, size, and SLL.

The length, width, and thickness of the proposed antenna are $110 \mathrm{~mm}, 31 \mathrm{~mm}$, and 1.3 mm, respectively [61]. The extra conductive layers are shown in pink color that establish a tapered CPW transition at each end. The length of the antenna was adjusted to achieve a proper directivity while keeping the antenna size reasonably small. The width of the antenna was also chosen to achieve single-mode propagation. To minimize the return loss, tapered via fences in addition to tapered microstrip transitions were implemented at the input/output of the antenna. Each end of the structure was terminated with a $2.4 \mathrm{~mm}$ connector, while one of them was terminated with a $50 \Omega$-matched load to absorb the remaining un-radiated power. The proposed LWA contains thirty-six transverse slots. A tapered CPW section was added at each end for SLL reduction. 


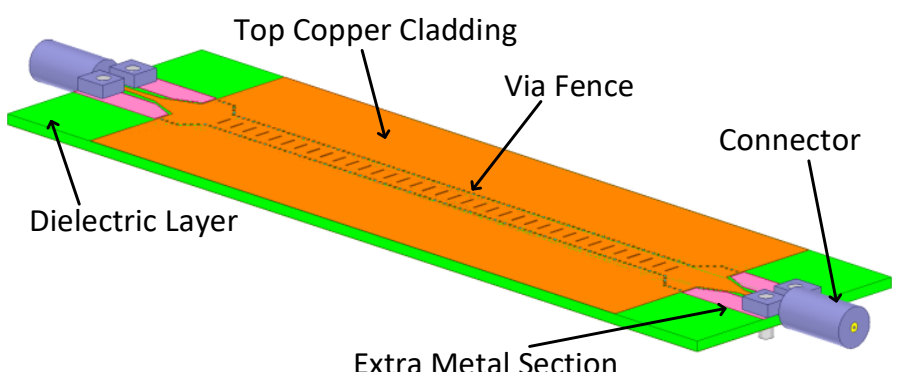

(a)

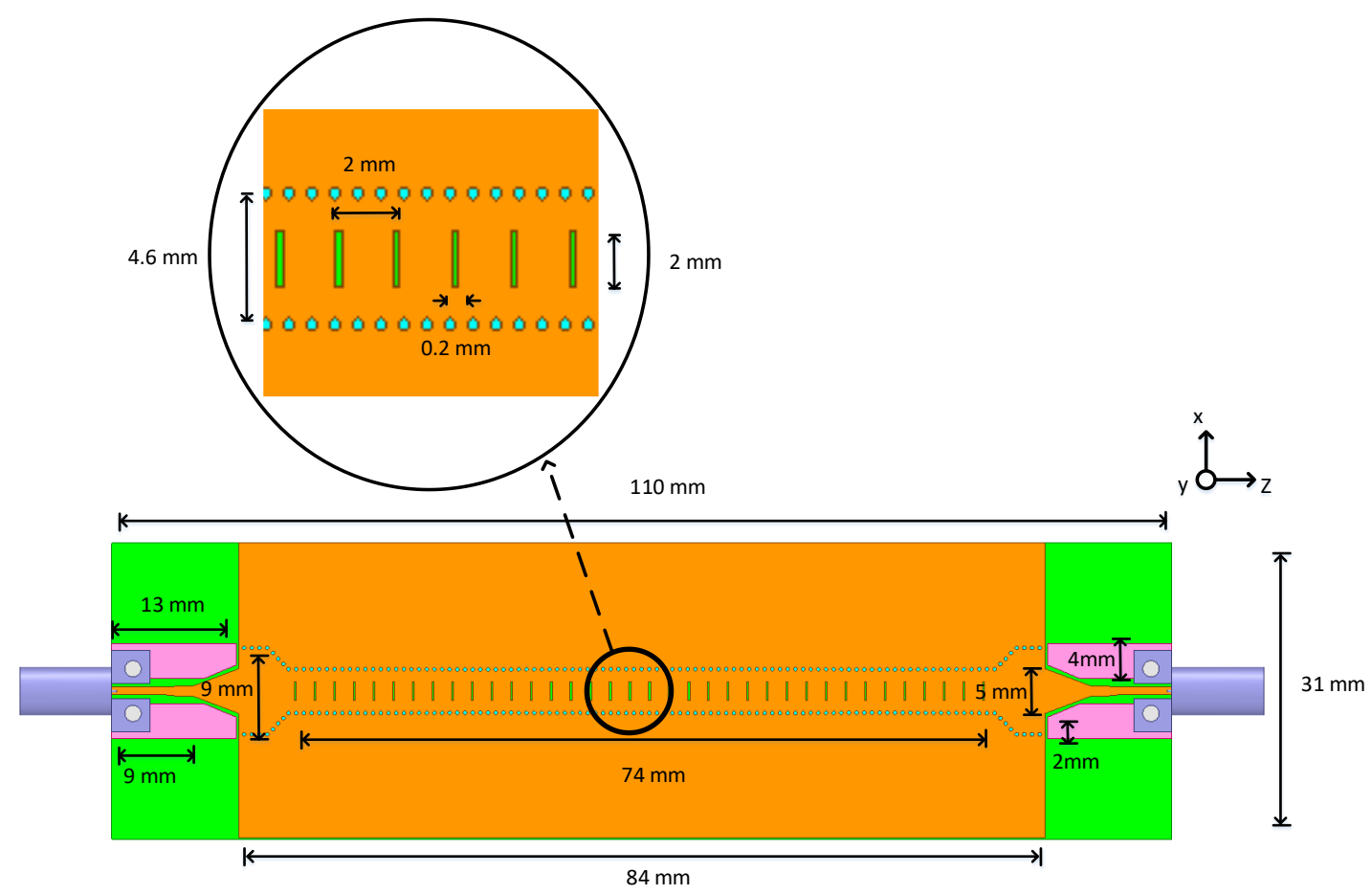

(b)

Figure 3.24. Schematic view of the proposed SLW LWA with reduced SLL. (a) 3D view, (b) top view [61].

\subsubsection{Simulated Results}

The simulation results are obtained using ANSYS HFSS v19.1. The S-parameters of the original and the modified antennas are presented in Fig. 3.25. The modified antenna has a return loss better than $10 \mathrm{~dB}$ over the frequency band from $27.1 \mathrm{GHz}$ to $28.9 \mathrm{GHz}$. It should be noted that the return loss was improved by modifying the feed transition. 
The normalized radiation patterns of the conventional and the modified designs at the E-plane (YZ-plane) are presented in Fig. 3.26, which confirm the SLL reduction by about 4.5dB. The peak realized gain and SLL of the proposed antenna are reported in Table 3.3, which indicates the medium gain and low SLL of the proposed design.

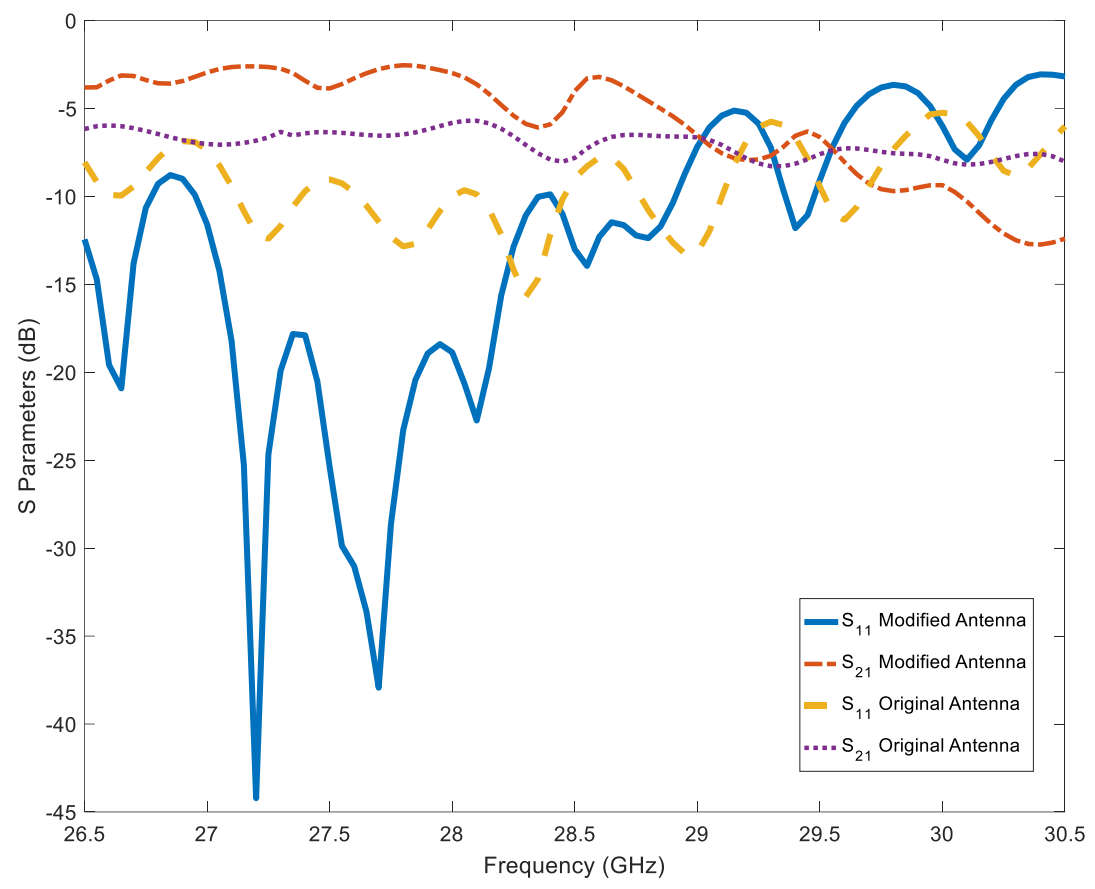

Figure 3.25. S-parameters of the modified fed SIW LWA and the conventional one [61].

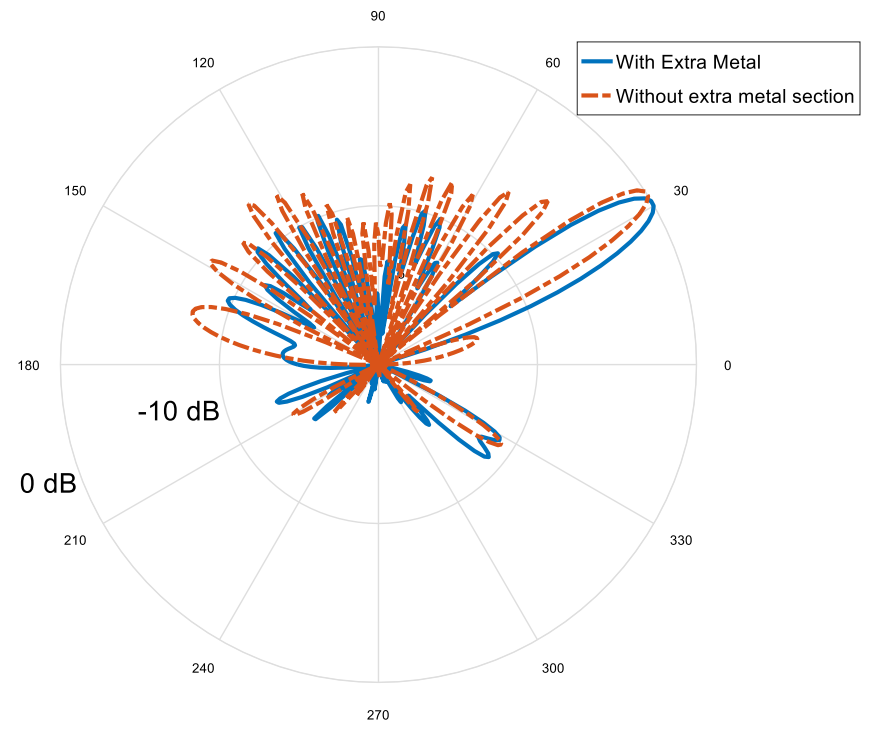

Figure 3.26. Normalized patterns of the conventional and the modified fed antennas at $28.5 \mathrm{GHz}$ [61]. 
Table 3.3. The peak realized gain and SLL of the modified fed and conventional SIW LWAs at 28.5 GHz [61].

\begin{tabular}{|c|c|c|}
\hline Antenna Types & Peak Realized Gain (dBi) & SLL (dB) \\
\hline Conventional SIW LWA & 10.2 & -4.5 \\
\hline Modified fed SIW LWA & 8.8 & -10.1 \\
\hline
\end{tabular}

\subsubsection{Practical Implementation and Measured Results}

To achieve a low-profile design, a SIW LWA was fabricated on a thinner $(0.5 \mathrm{~mm})$ Rogers RT/Duroid 5880 substrate using the introduced design method in Section 3.3.1. It should be noted that different taper CPWs were implemented in this antenna because of the smaller thickness than the designed antenna in Section 3.3.1. The radiation length of the antenna was determined by (2.4). As stated in Section 3.3.1, the total length of the antenna, including the feed, was optimized to improve the impedance matching. The width of the SIW (w) was calculated by (2.5) and (2.6) to accomplish a single-mode ( $\left.\mathrm{TE}_{10}\right)$ propagation. In other words, the dimensions were selected to obtain $\mathrm{TE}_{10}$ mode with the cut-off frequency of $24 \mathrm{GHz}$. As stated before, the via fence confines the fields when $s / d$ and $d / w$ be smaller than 3 and 0.2 , respectively [35]. Hence, choosing $s=d=$ $0.4 \mathrm{~mm}$ results in $w=4.6 \mathrm{~mm}$. The total length, width, and thickness of the antenna are $110 \mathrm{~mm}, 31 \mathrm{~mm}$, and $0.5 \mathrm{~mm}$, respectively. A summary of the geometrical parameters of the antenna is reported in Table 3.4. The fabricated and schematic view of the proposed LWA are presented in Figs. 3.27(a), (b), (e). To demonstrate the undesired effect of the feed transition on the SLL, conventional SIW LWAs with microstrip transition and ideal waveport are also investigated, as demonstrated in Figs. 3.27(c) and (d). The proposed LWA consists of thirty-six transverse slots to achieve proper gain. The slots dimensions 
were selected such that the ratio of $L_{s} / W_{s}$ was set at 10 to control the leakage rate [28]. The period of the slots was chosen to achieve single Floquet mode propagation.

The proposed LWA was fed using surface-mounted connectors and tapered transitions because of its low-profile. As stated before, microstrip transition results in unwanted radiation [142] and large SLL. Similar to Section 3.3.1, tapered conductive sections were located alongside the microstrip transitions to reduce SLL. The extra conductive sections were grounded through the screws of the connectors that resemble a CPW. The dimensions of each extra conductive section (i.e., $L_{m f}$ and $W_{m f}$ ) were optimized using fullwave simulation along with the physical intuition to minimize SLL. Tapering the CPW leads to a more uniform field transition to the slots, resulting in a lower SLL. In other words, the field transition into the slot region became more uniform, which contributed to the reduction of unwanted radiation from the feed and lower SLL. It should be noted that modification of the radiating section containing transverse slots was intentionally avoided to address the undesired radiation from the microstrip transition.

Table 3.4. A summary of the geometrical parameters of the antenna [62].

\begin{tabular}{|c|c|c|c|c|c|c|c|}
\hline Parameter & $h$ & $W$ & $W_{t}$ & $W_{s}$ & $L_{s}$ & $L_{m f}$ & $W_{m f}$ \\
\hline Value (mm) & 0.5 & 4.6 & 31 & 0.2 & 2 & 13 & 3.7 \\
\hline
\end{tabular}




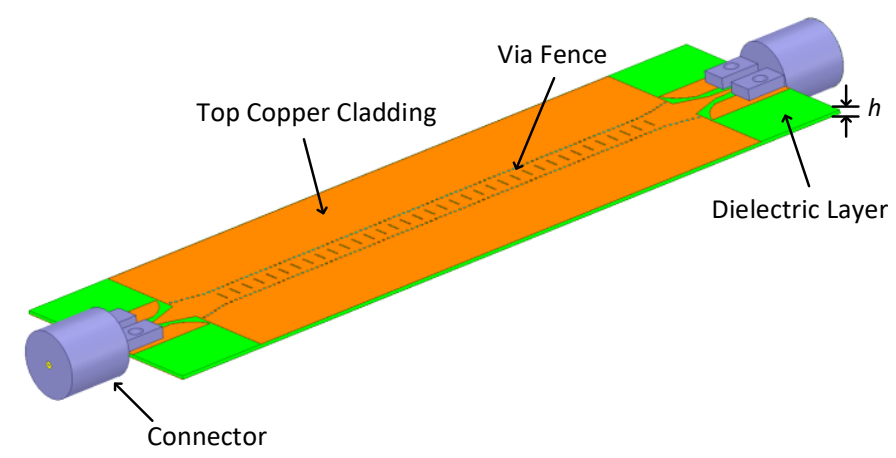

(a)

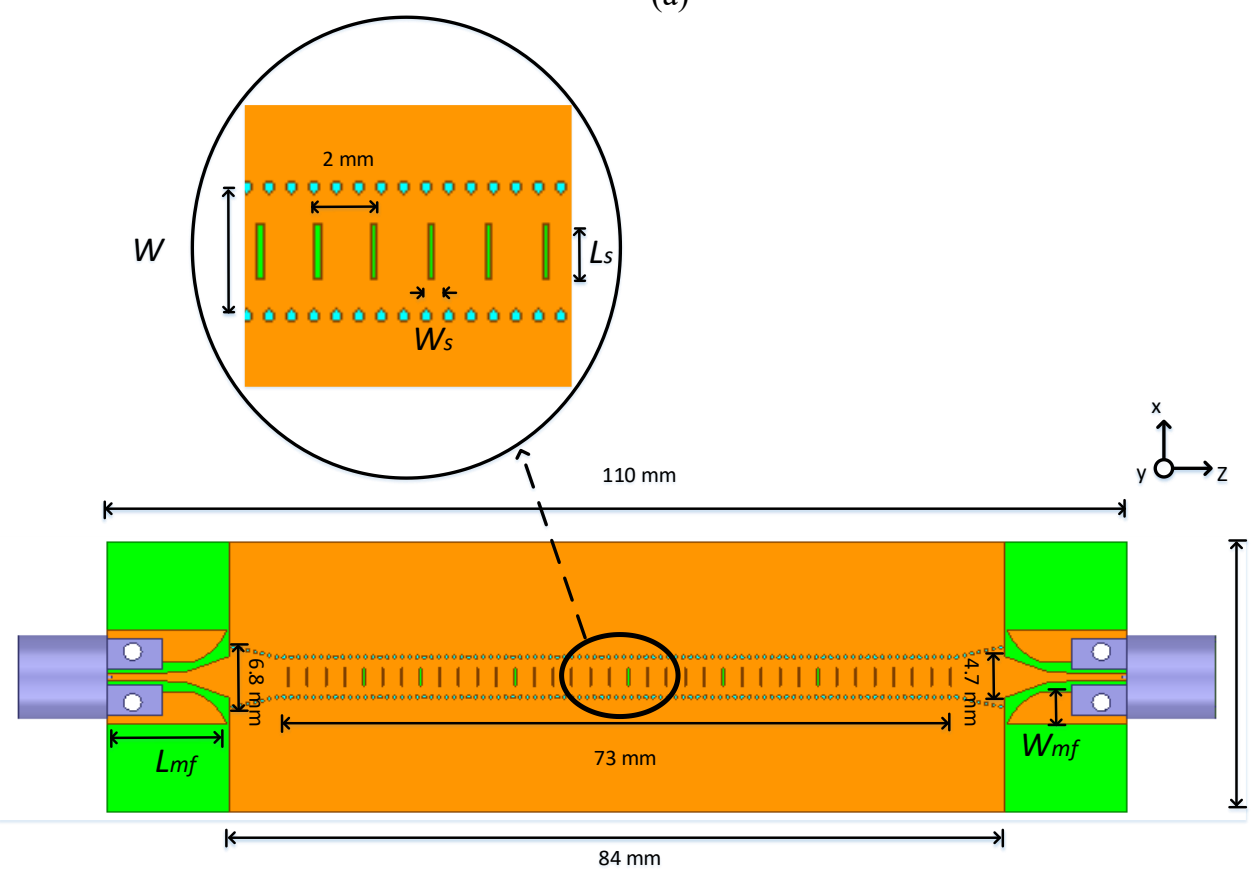

Wt

(b)

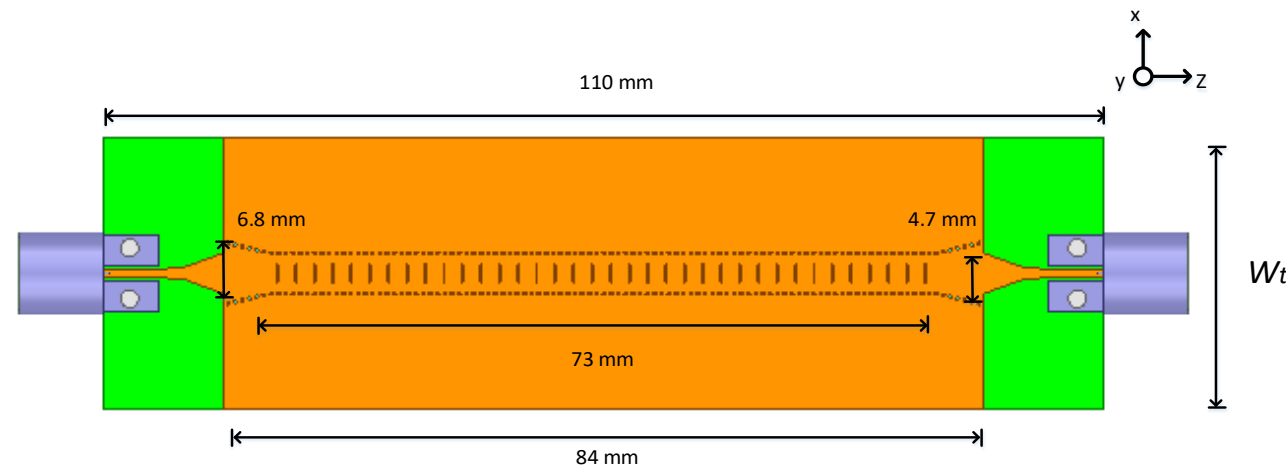

(c)

Figure 3.27. The structure of the SIW LWA [62]. (a) 3D View, (b) top view of the LWA with the proposed feed structure, (c) top view of the conventional LWA (i.e., the antenna fed by microstrip line). 


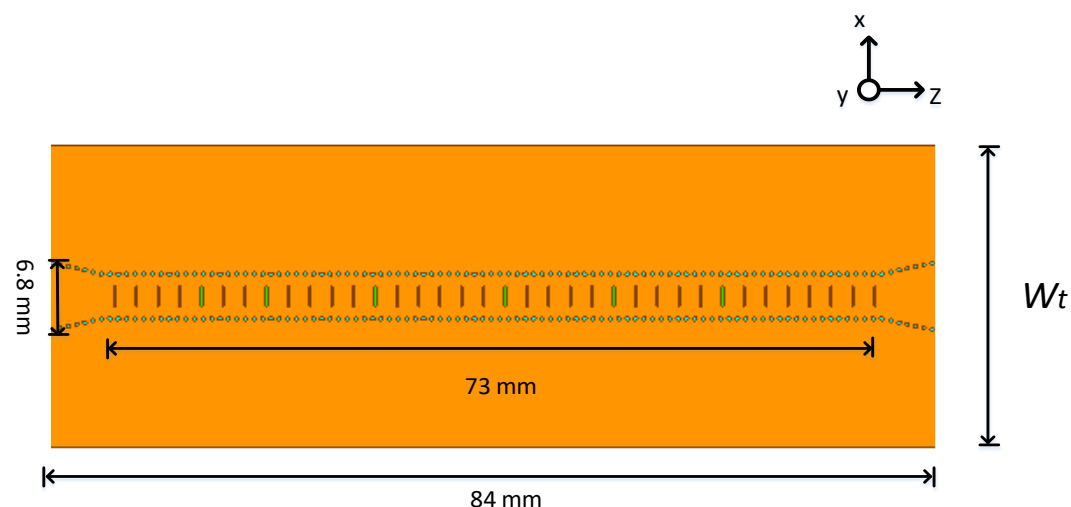

(d)

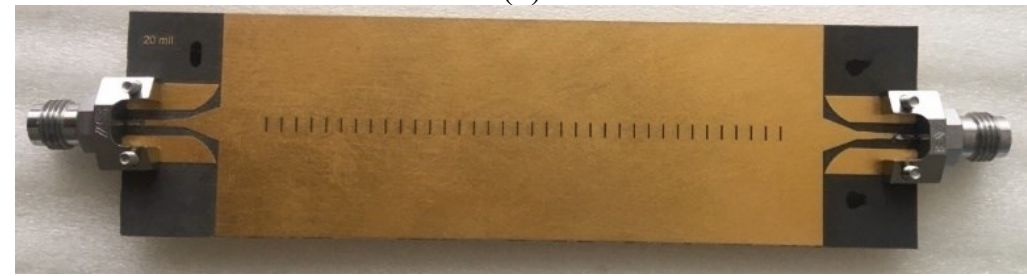

(e)

Figure 3.27. Schematic view of the fabricated SIW LWA [62] (cont'd). (d) top view of the antenna without the feeds (i.e., the same LWA fed through ideal waveport), (e) fabricated antenna.

To minimize the return loss, the via fences were tapered by moving from the center toward input/output ports. Each end of the tapered CPW feed was terminated with a $2.9 \mathrm{~mm}$ connector. Generally, the LWA was designed with the maximum achievable radiation efficiency of $90 \%$. This is because when the radiation efficiency surpasses $90 \%$, the LWA becomes unstable, which is not realistic [28]-[30]. One of the $2.9 \mathrm{~mm}$

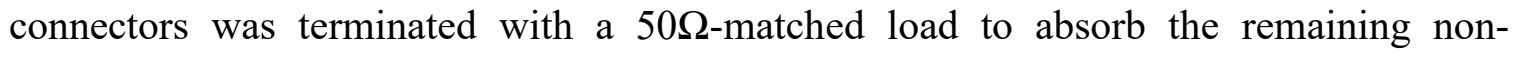
radiated power.

S-parameters were measured using a Rohde \& Schwarz ZVA-67 VNA. The radiation gain patterns of the proposed antenna were measured at the anechoic chamber using the MI-12A-26 standard gain horn antenna. The measurement setup is illustrated in Fig. 3.28. The measured and simulated S-parameters of the tapered CPW antenna are presented in Fig. 3.29, exhibiting return loss better than $10 \mathrm{~dB}$ over the frequency band from $26 \mathrm{GHz}$ to $30 \mathrm{GHz}$. The corresponding insertion loss is about $8 \mathrm{~dB}$, exhibiting the low loss. 


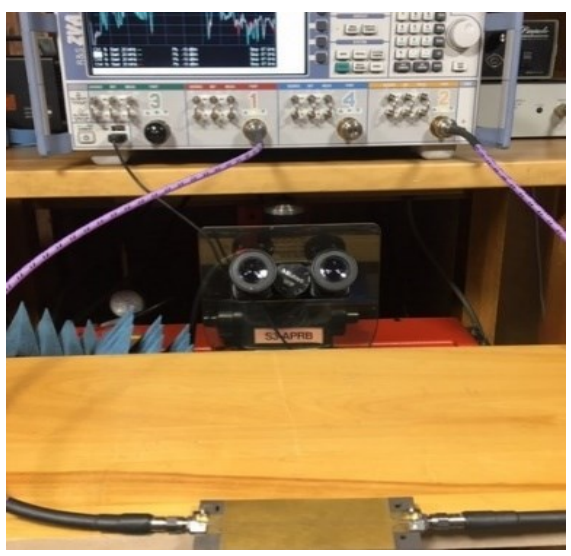

(a)

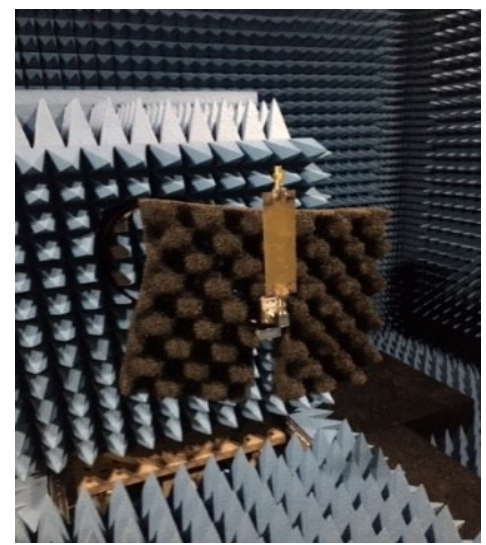

(b)

Figure 3.28. Measurement setup. (a) Antenna under test connected to VNA, (b) Antenna under test in the anechoic chamber [62].

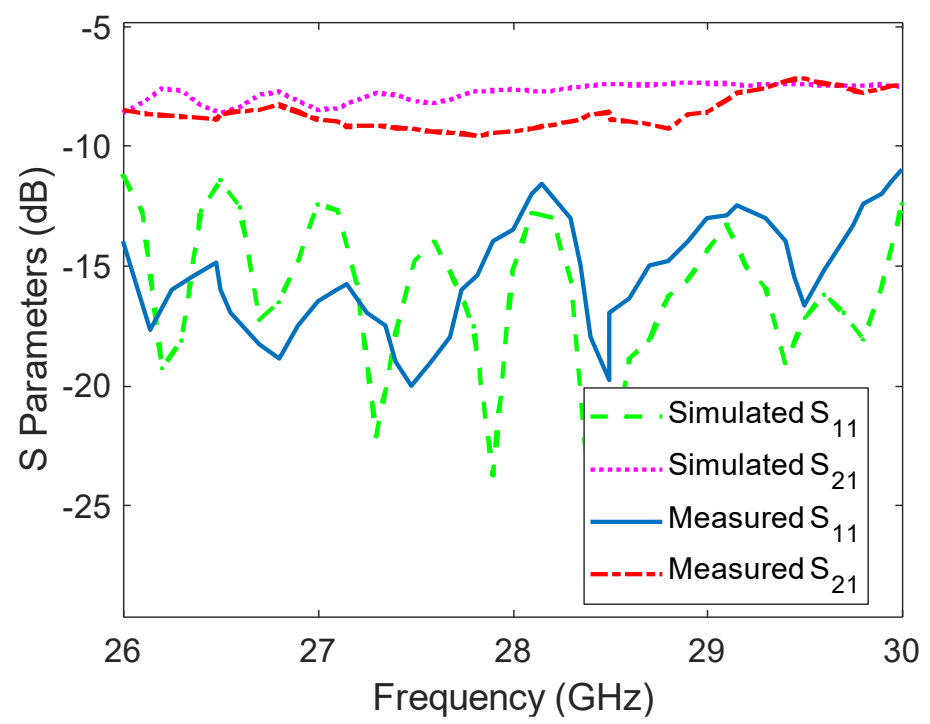

Figure 3.29. S-parameters of the fabricated SIW LWA with reduced SLL [62].

The measured and simulated gain radiation patterns of the proposed LWA along with the radiation patterns of a SIW LWA fed by conventional microstrip transition (shown in green) and ideal waveport (shown in purple) at $28.5 \mathrm{GHz}$ are demonstrated in Fig. 3.30. This confirms the reduction of SLL by introducing the tapered CPWs to the input and the output. According to Fig. 3.30, implementing ideal waveports, representing only the contribution of transverse slots to the radiation pattern, results in the lowest SLL. However, it is not realistic since the low-profile antenna can only be excited through 
$\mathrm{CPW} /$ microstrip transitions. A marked improvement of SLL is observed by comparing the measured results of CPW fed SIW LWA to the similar microstrip fed SIW LWA. The measured peak realized gain and SLL are $6.1 \mathrm{dBi}$ and $-11.4 \mathrm{~dB}$, respectively. It should be noted that the small thickness of the antenna results in a high conduction loss [137], which leads to the reduction of gain.

Generally, the SLL is influenced by the radiation from the feed and the main radiating aperture. The feed is the first stage of the antenna encountering the incoming signal. As a result, it has a significant impact on the radiation pattern, as shown in Fig. 3.30. Introducing additional conducting sections adjacent to the microstrip transition is reminiscent of loading the microstrip transition with capacitive loads leading to the variations in parasitic capacitance and propagation constant. Doing so results in the SLL reduction. Tapering period of slots [53], slots sizes, and via fences [52], [54] leads to further SLL reduction.

Radiation patterns of the proposed SIW LWA at different frequencies are illustrated in Fig. 3.31, indicating that the antenna scans space with moderate gain and low SLL over the frequency band of $28-29 \mathrm{GHz}$. According to Fig. 3.31 , a $16^{\circ}$ frequency beamscanning range was achieved in the frequency band of 28-29 GHz. Therefore, our proposed LWA is a suitable candidate for $5 \mathrm{G}$ beam-scanning applications such as V2X communications. Moreover, the frequency beam-scanning capability of our proposed LWA can be used as a fine-tuning mechanism to compensate for the unwanted operational and environmental variations. 


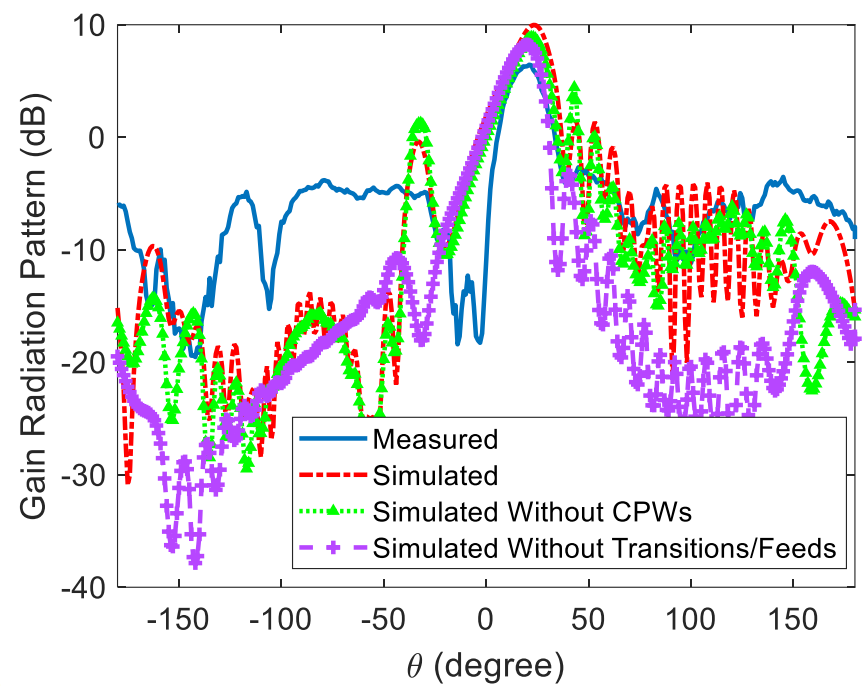

Figure 3.30. Measured and simulated radiation patterns of the proposed antenna at $28.5 \mathrm{GHz}$ and Eplane (YZ plane) [62].

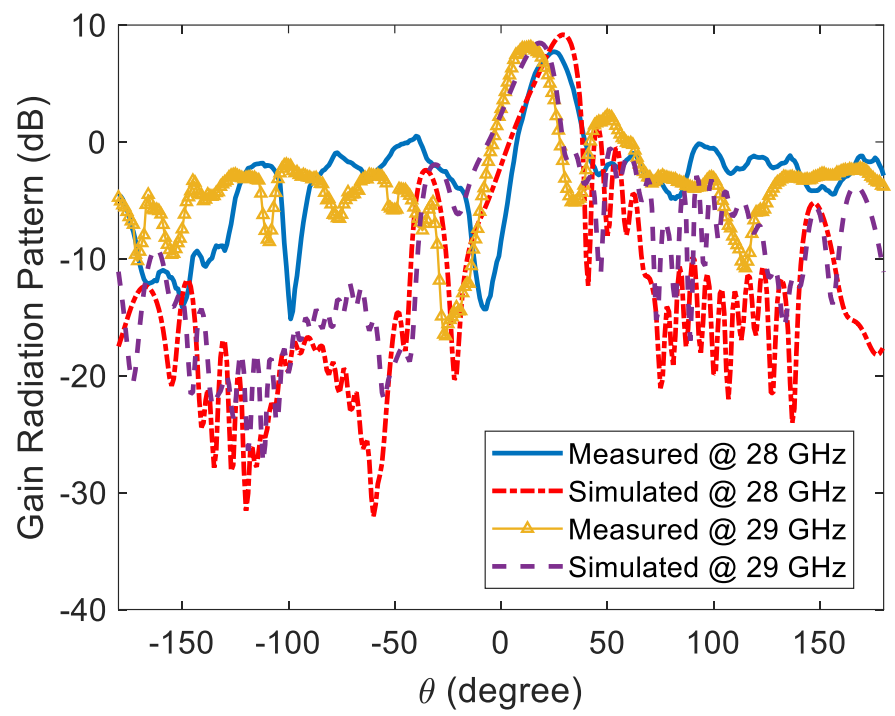

Figure 3.31. Measured and simulated radiation patterns of the proposed LWA at 28 and $29 \mathrm{GHz}$ [62].

A parametric study was carried out to investigate the discrepancies between measured and simulated results in more detail. Several parameters were changed individually, including the thickness of the substrate $(h)$, the SIW width $(W)$, slots sizes $\left(L_{s}\right.$ and $\left.W_{s}\right)$, and the size of the modified feed section $\left(L_{m f}\right.$ and $\left.W_{m f}\right)$. It should be noted that in each case, only one parameter was changed, while the others stated in Table 3.4 were held 


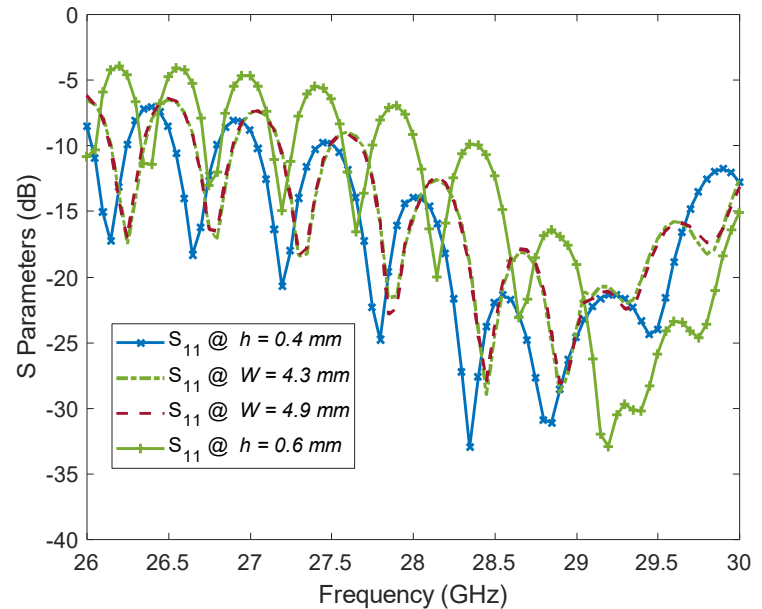

(a)

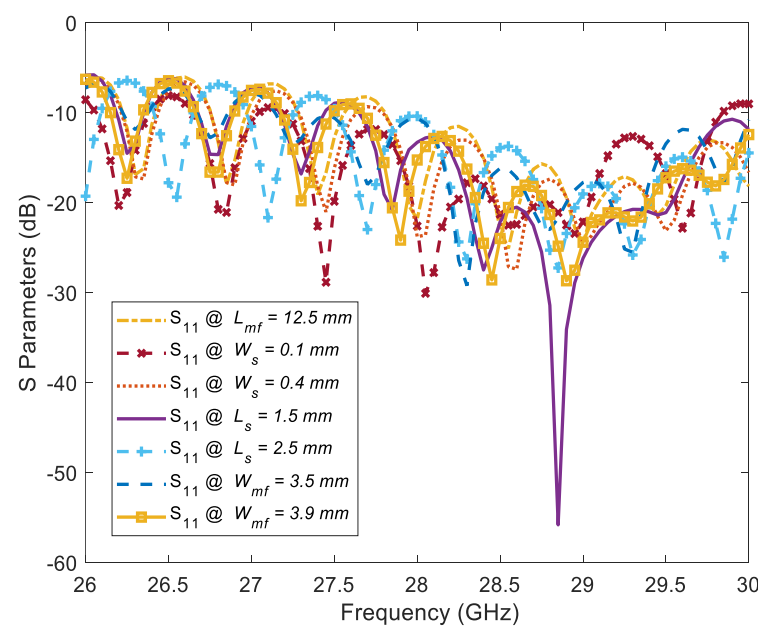

(c)

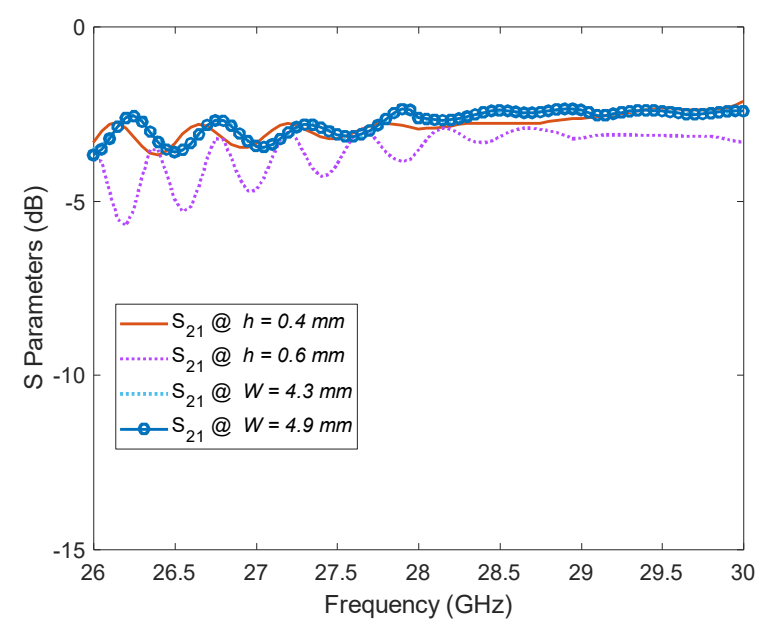

(b)

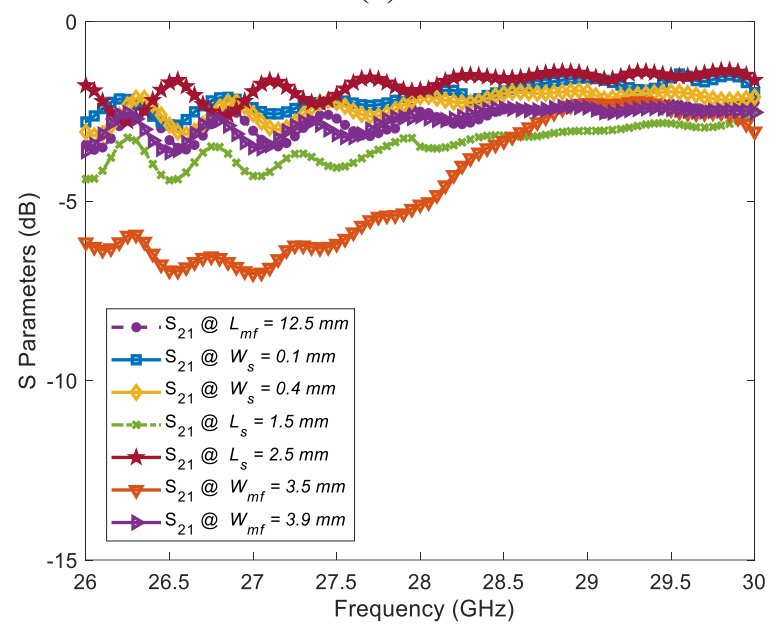

(d)

Figure 3.32. S-parameters of the proposed SIW LWA with different parameters [62]. (a) $S_{11}$ at different values of $h$ and $W$. (b) $S_{21}$ at different values of $h$ and $W$. (c) $S_{11}$ different values of $L_{s}, W_{s}$, $L_{m f}$, and $W_{m f}$. (d) $S_{21}$ different values of $L_{s}, W_{s}, L_{m}$, and $W_{m f}$.

constant. The S-parameters for different combinations are reported in Fig. 3.32. It should be noted that changing $W$ leads to variations in the cut-off frequency $\left(f_{c}\right)$ of the propagating modes. Hence, as observed in Fig. 3.32, $W$ has a major effect on the Sparameters. The normalized radiation patterns corresponding to different combinations of geometrical parameters are illustrated in Fig. 3.33. According to Fig. 3.33, $L_{s}, W_{s}$, and $W$ play the dominant roles in determining SLL and main-lobe pointing angle $\left(\theta_{0}\right)$. This is because slot size controls the leakage rate, while $W$ has a dominant effect on $f_{c}$ and field 
confinement. Furthermore, changing $L_{s}, W_{s}$, and $W$ results in variations of the intensity of the beams radiating underneath the antenna. Therefore, fabrication errors in slot size and SIW width lead to discrepancies between simulated and measured radiation patterns. The peak realized gain of the antenna for different parameters is reported in Fig. 3.34, indicating that $L_{s}, W_{s}$, and $W$ have dominant effects on the peak realized gain.

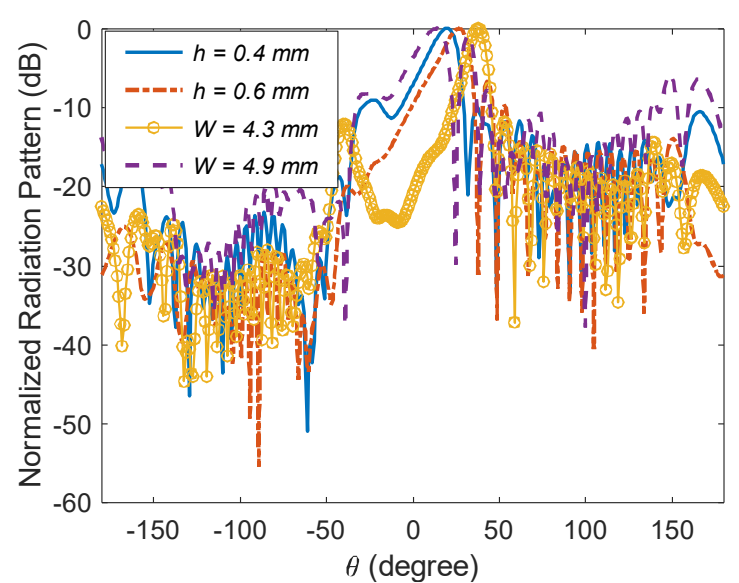

(a)

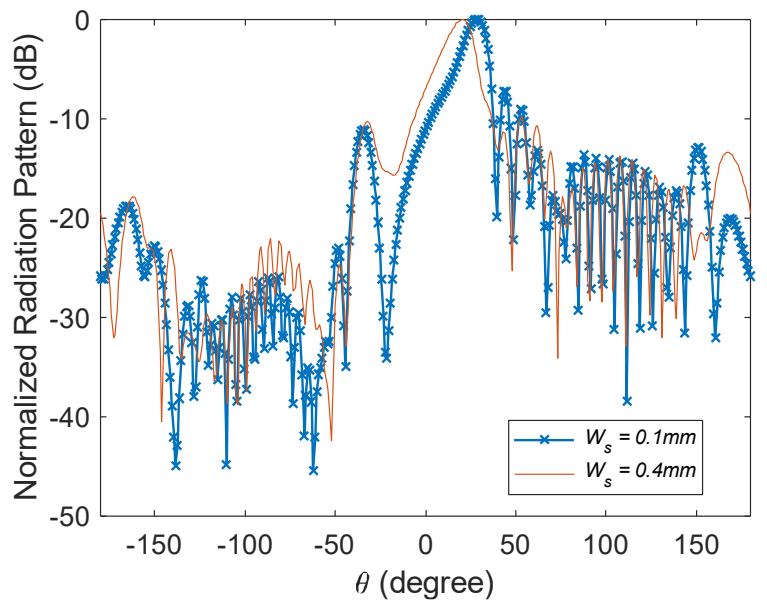

(c)

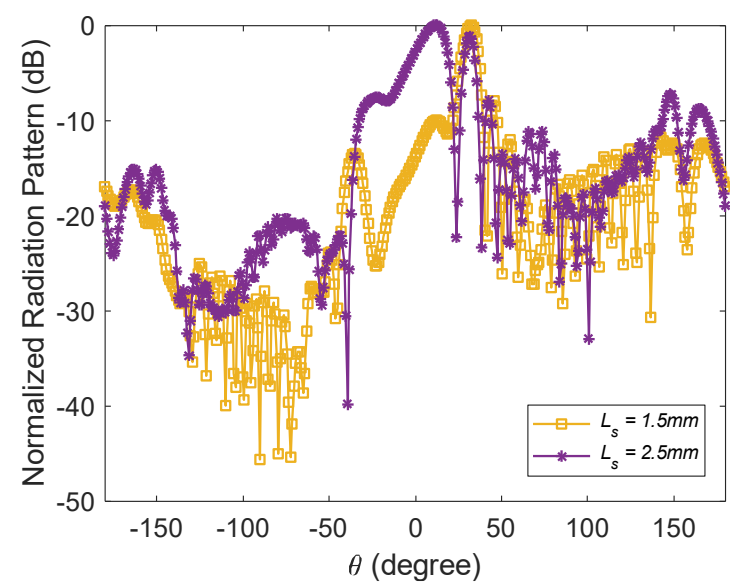

(b)

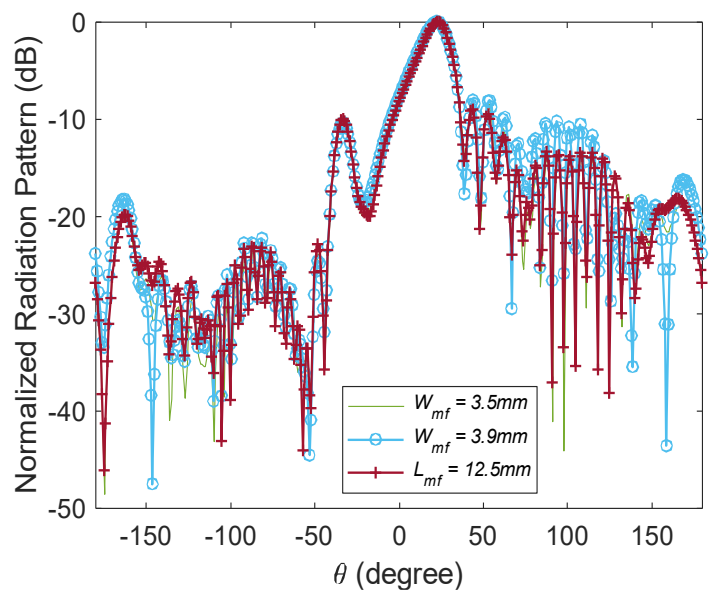

(d)

Figure 3.33. Normalized radiation patterns of the proposed antenna obtained by changing different parameters individually at 28.5 GHz [62]. (a) E-plane for different values of $h$ and $W$, (b) E-plane for different values of $L_{s}$, (s) E-plane for different values of $W_{s}$, (d) E-plane for different values of $L_{m f}$, and $\boldsymbol{W}_{\boldsymbol{m}}$. 


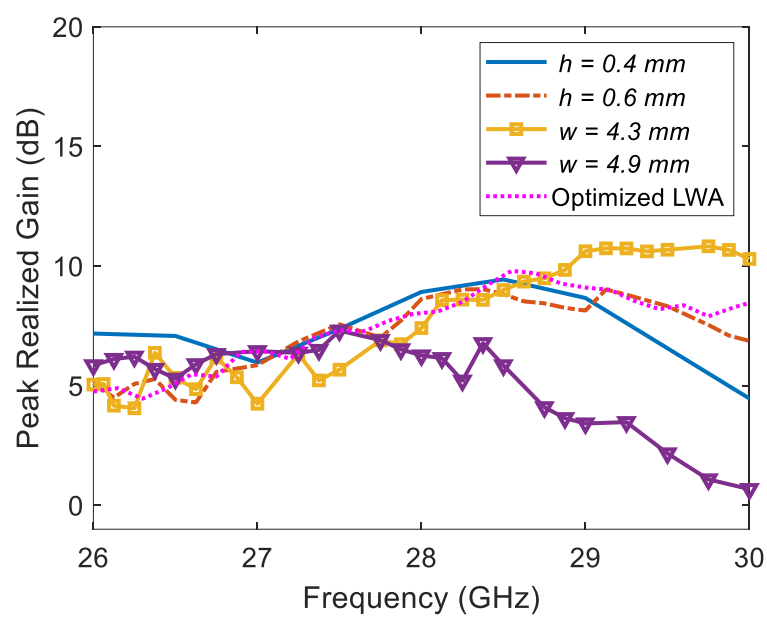

(a)

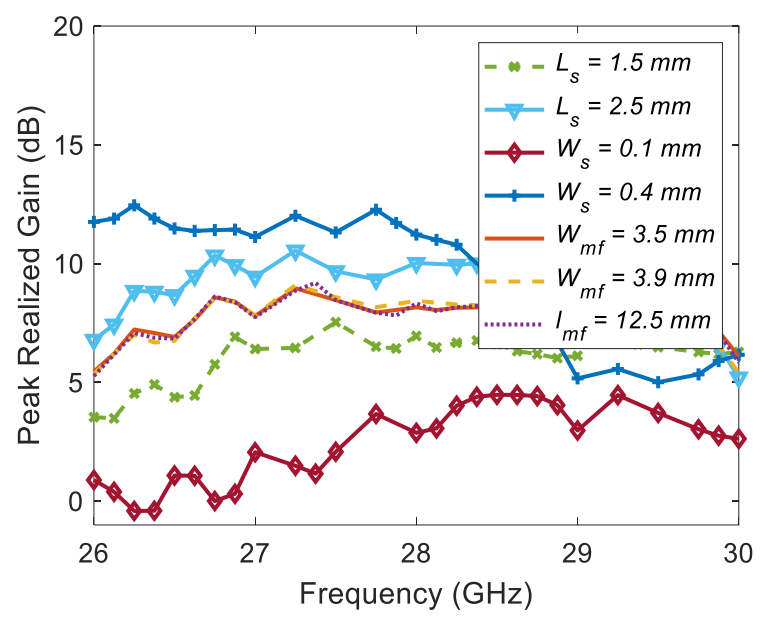

(b)

Figure 3.34. Peak realized gain diagrams of the modified fed antenna corresponding to different parameters [62]. (a) different values of $h$ and $W$. (b) different values of $L_{s}, W_{s}, L_{m}$, and $W_{m f}$.

To investigate the effect of the ground plane's size, the total width of the antenna $\left(W_{t}\right)$ was changed, while the other parameters stated in Table 3.4 were held constant. The corresponding S-parameters, normalized radiation patterns, and peak realized gain are reported in Figs. 3.35-3.37. According to Figs. 3.35-3.37, changing $W_{t}$ results in small variations in SLL, return loss, and peak realized gain. This shows that the antenna has good fabrication tolerance concerning $W_{t}$.

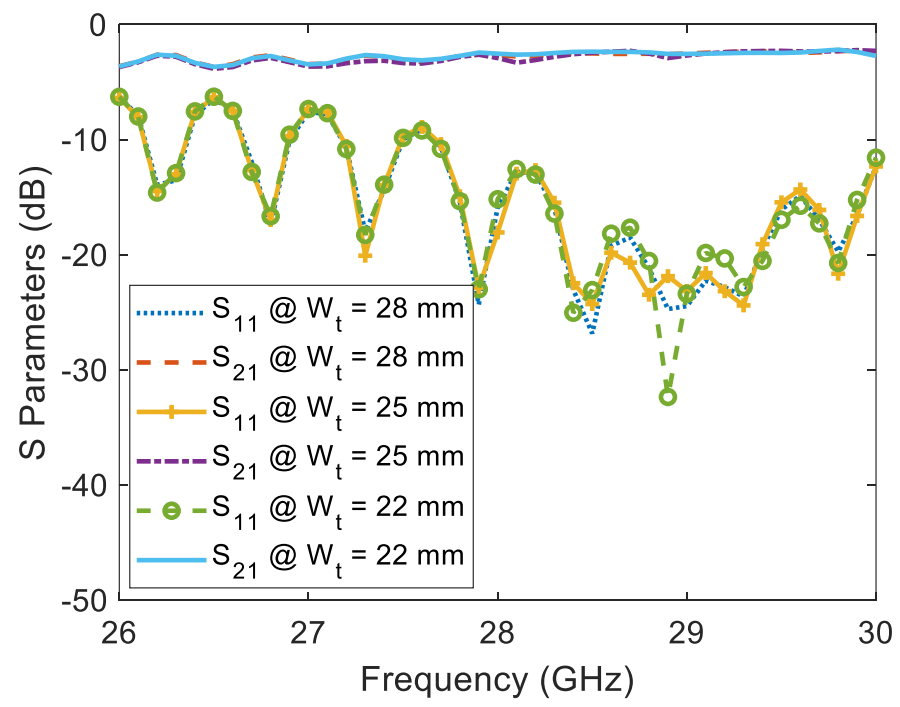

Figure 3.35. S-parameters of the proposed SIW LWA with different $W_{t}[62]$. 


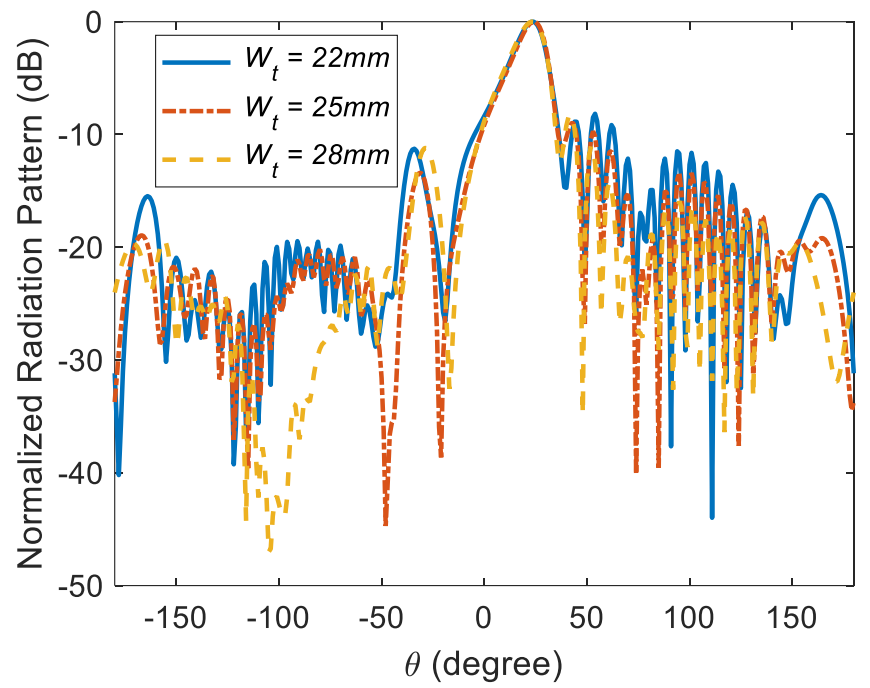

Figure 3.36. Normalized radiation patterns of the SIW LWA with different $W_{t}$ at $28.5 \mathrm{GHz}$ [62].

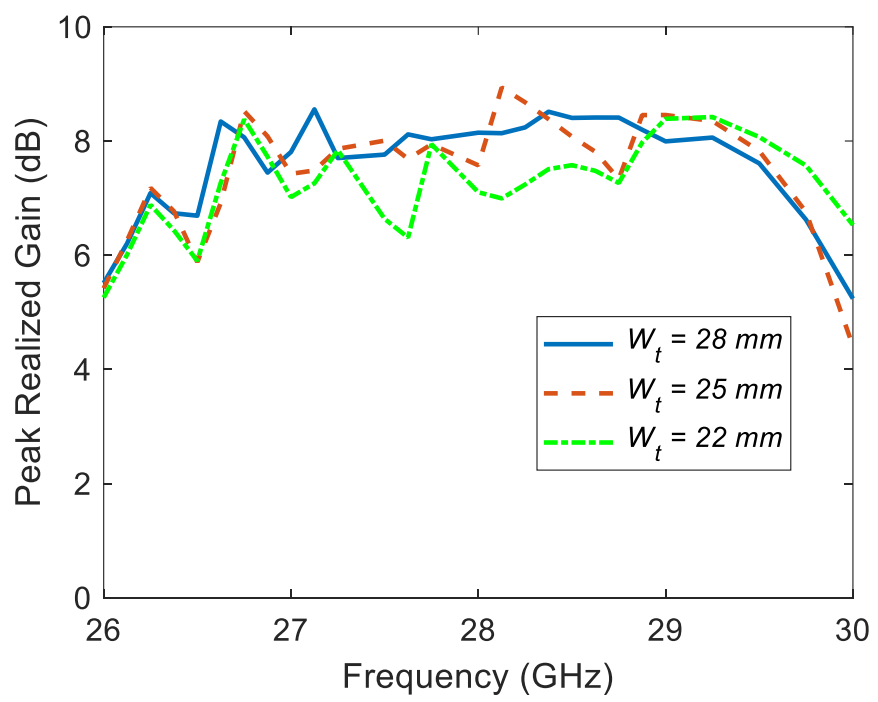

Figure 3.37. Peak realized gain diagrams of the modified fed antenna corresponding to different $W_{t}$

[62].

To investigate the discrepancies further, we rotated the connectors by $\xi$ from the horizon (XZ plane), similar to the antenna presented in Fig. 3.16. As stated before, attaching the connectors can bend both ends of the antenna due to the small thickness of the board. Moreover, connectors may not be attached properly, and the pin may not lie completely on the signal trace. To investigate the impact of the misalignment of the connectors, antenna performance was monitored by varying $\xi$ from $-10^{\circ}$ to $20^{\circ}$. The S- 
parameters, normalized radiation patterns, and peak realized gain of the antenna for $\xi=$ $10^{\circ}, 10^{\circ}$, and $20^{\circ}$ are reported in Figs. 3.38-3.40, respectively. According to Fig. 3.39, changing $\xi$ degrades SLL severely, mainly due to the defective connection between the pin and the signal trace. Fig. 3.39 also indicates that rotating the connectors increases the intensity of the beams radiating underneath the antenna.

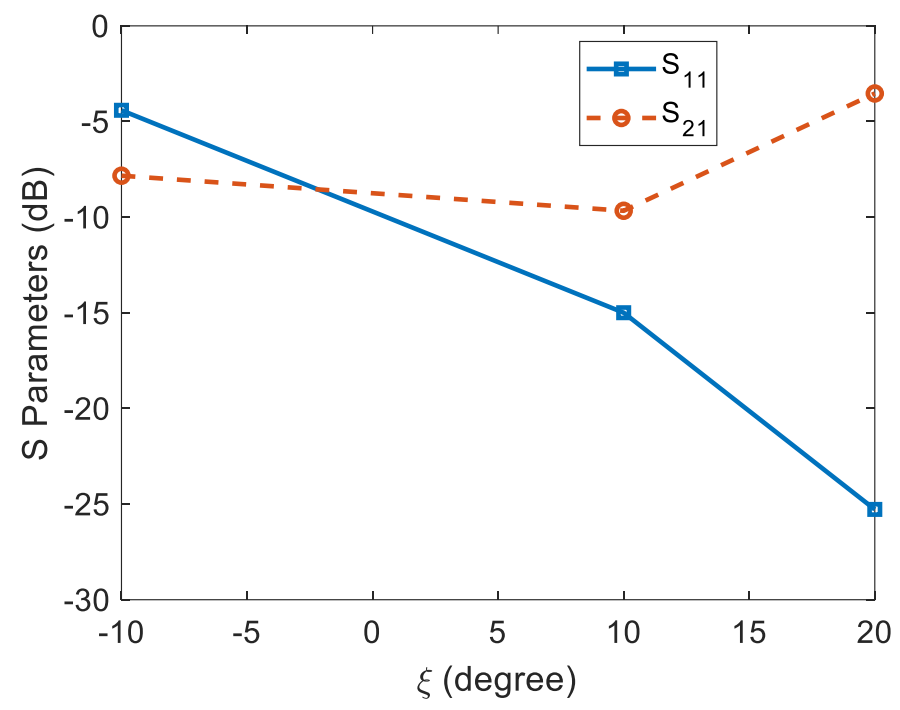

Figure 3.38. S-parameters of the proposed modified fed LWA with rotated connectors at $28.5 \mathrm{GHz}$ [62].

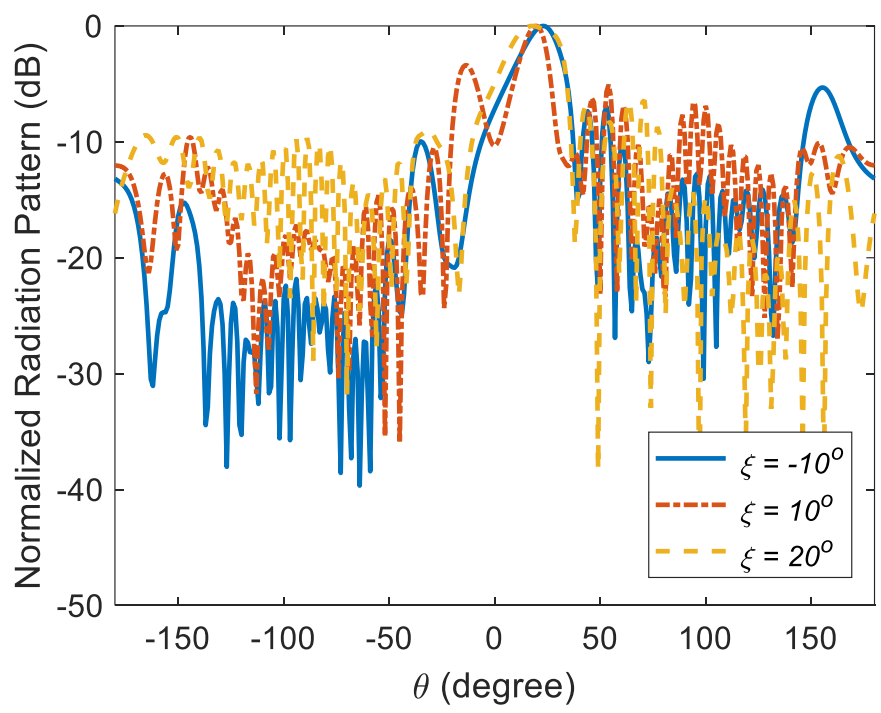

Figure 3.39. Normalized radiation patterns of the proposed SIW LWA with offset connectors at 28.5 GHz and E-plane [62]. 


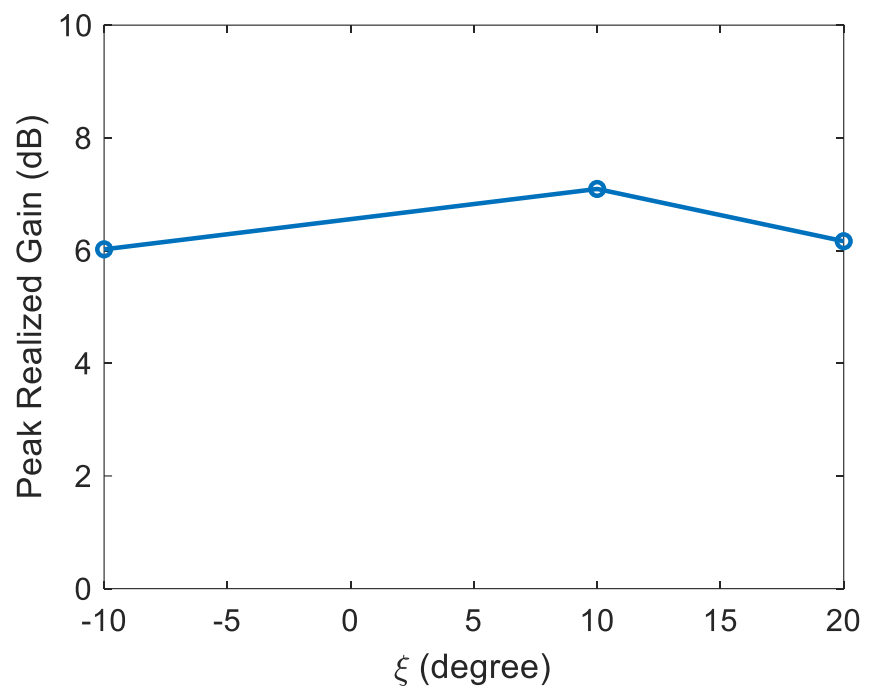

Figure 3.40. Peak realized gain of the proposed modified fed LWA with offset connectors at $28.5 \mathrm{GHz}$ [62].

As stated before, the antenna may bend under mechanical stress due to the small thickness of the board. The antenna was bent to examine such a case, as illustrated in Fig. 3.41. It is similar to gradually changing the vertical distance from the horizon up to $h_{b}=6$ $\mathrm{mm}$ by moving from the center toward the connectors. The S-parameters, normalized radiation patterns, and peak realized gain of the bent antenna are reported in Figs. 3.42-3.44, respectively. According to Figs. 3.42-3.44, bending the board results in severe degradations of the antenna responses in terms of SLL, return loss, and peak realized gain.

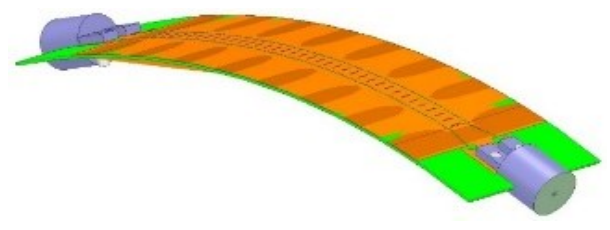

(a)

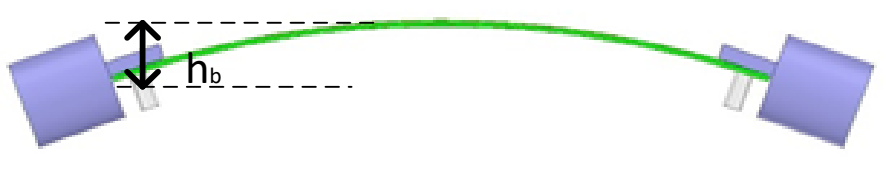

(b)

Figure 3.41. Schematic view of the bent SIW LWA [62]. (a) 3D view, (b) side view. 


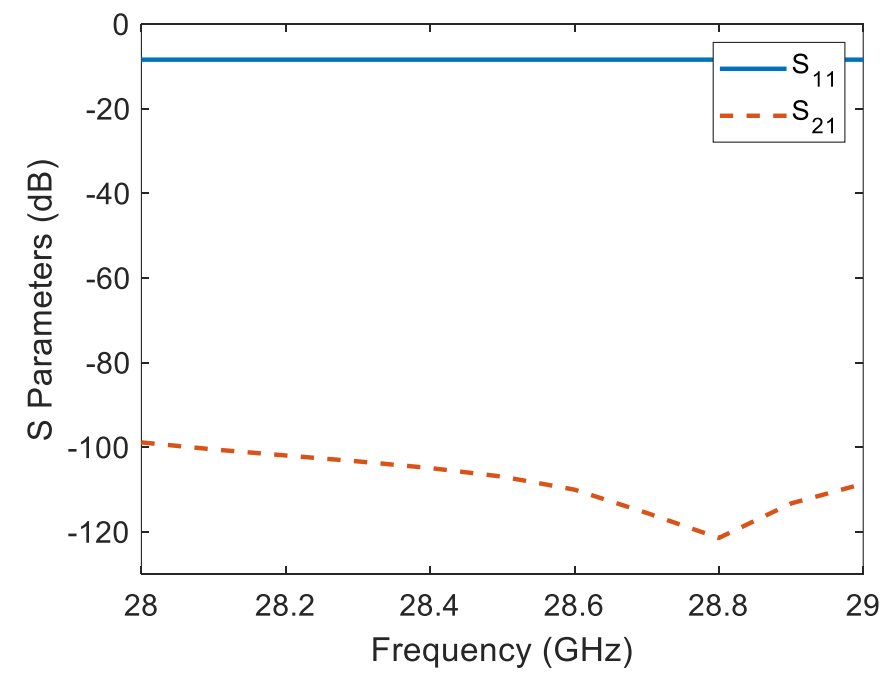

Figure 3.42. S-parameters of the proposed bent LWA [62].

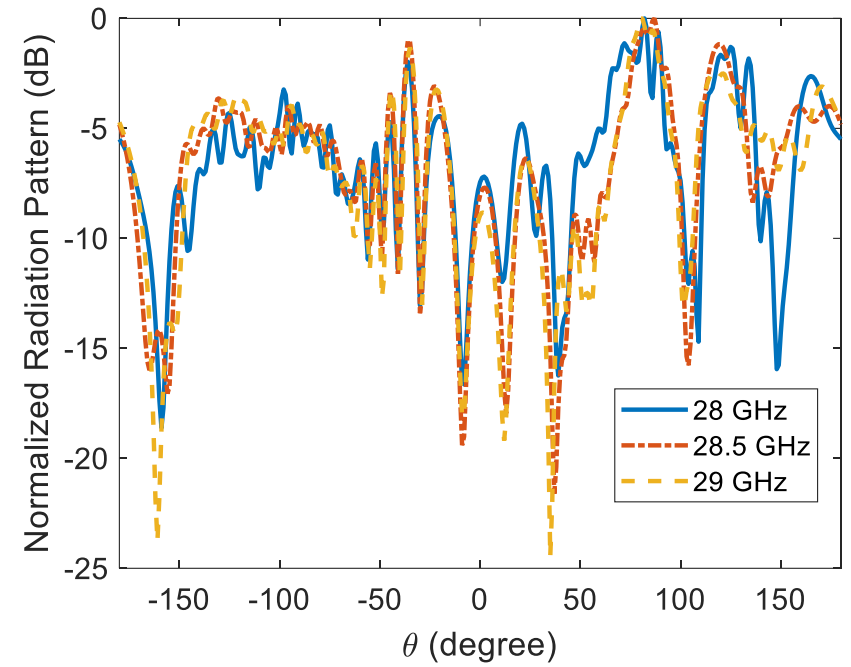

Figure 3.43. Normalized radiation patterns of the proposed bent SIW LWA [62].

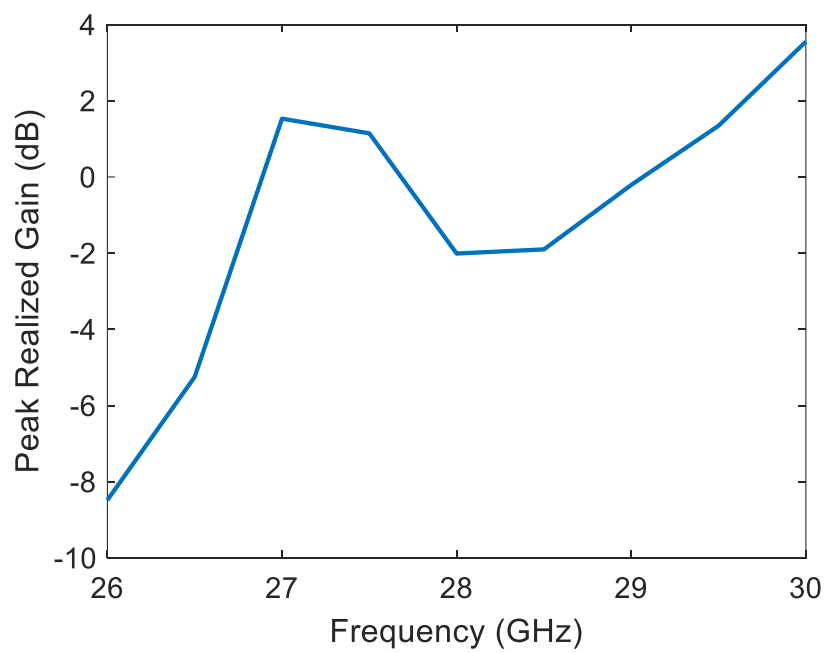

Figure 3.44. Peak realized gain of the proposed bent modified fed LWA [62]. 
According to the sensitivity analysis, changing $\xi, L_{s}, W_{s}$, and $W$ contribute the most to the discrepancies. In other words, the discrepancies between measurements and simulation results are mostly due to errors in the assembly of the connectors and fabrication errors in slots sizes and locations of via fences. The board curvature due to the mechanical stress is another factor of discrepancies. Furthermore, the air gap in the via fence may cause additional discrepancies between measured and simulated results.

A summary of methods for reducing SLL of LWAs is presented in Table 3.5. From the fabrication perspective, modifying the feed section is easier than tapering via fences [52], slots sizes and shapes [52], [54], [55], [160], and period of slots [53]. The radiating section (i.e., via fence and slots) of the antennas were optimized for achieving minimum SLL in [34], [52]-[55], [71], and [160]-[165], while SLL of our proposed CPW tapered fed LWA was reduced by modifying the feed rather than the radiating slotted section. This is the key distinct feature of the proposed design as compared to [34], [52]-[55], [71], and [160]-[165]. The reported SLL in [52]-[54] and [71] are lower than the one presented here. It should be noted that the reported antennas in [34], [52], and [53] were fed by coaxial through-hole connectors, which is not a practical feeding mechanism for the low-profile antenna. In other words, the feeding mechanisms in [34], [52], and [53] are different from the one investigated in Section 3.3. The undesired radiation from the microstrip transition was also ignored in [54], [55], [71], [160]-[165]. While the feed, in addition to the slotted radiating section, plays a major role in SLL. As stated before, tapering the radiation section was purposely avoided in Section 3.3 to fully understand the effect of the feed on SLL. Therefore, it is possible to further reduce SLL by modifying the radiating section and the feed simultaneously. 
Table 3.5. Comparison between different methods of SLL improvement [62].

\begin{tabular}{|c|c|c|c|c|c|}
\hline Reference & Methodology & $\begin{array}{l}\text { SLL } \\
\text { (dB) }\end{array}$ & $\begin{array}{c}\text { Peak Realized Gain } \\
\text { (dBi) }\end{array}$ & Antenna Length & $\begin{array}{l}\text { Frequency Band } \\
\text { (GHz) }\end{array}$ \\
\hline $\begin{array}{c}\text { Our } \\
\text { proposed } \\
\text { SIW LWA } \\
\text { with } \\
\text { tapered } \\
\text { CPW feed }\end{array}$ & $\begin{array}{l}\text { Adding extra } \\
\text { metal } \\
\text { sections } \\
\text { besides the } \\
\text { main } \\
\text { microstrip } \\
\text { line }\end{array}$ & -11.4 & 6.1 & $10 \times \lambda$ & $26-30$ \\
\hline [52] & $\begin{array}{l}\text { Tapering via } \\
\text { fence \& slots }\end{array}$ & -21 & 13 & $13 \times \lambda$ & $10.84-12$ \\
\hline [54] & $\begin{array}{l}\text { Tapering } \\
\text { slots }\end{array}$ & -14 & 10 & $8 \times \lambda$ & $9.8-12.5$ \\
\hline [55] & $\begin{array}{l}\text { Tapering the } \\
\text { via fence and } \\
\text { slots }\end{array}$ & -11 & 8 & $7 \times \lambda$ & $9-10.7$ \\
\hline [53] & $\begin{array}{c}\text { Tapering } \\
\text { period of } \\
\text { slots }\end{array}$ & -20 & 13.87 & $10 \times \lambda$ & $9.2-10.8$ \\
\hline [71] & $\begin{array}{l}\text { Tapering } \\
\text { interdigital } \\
\text { slots at the } \\
\text { aperture of } \\
\text { HMSIW }\end{array}$ & -15 & 14.5 & $5.6 \times \lambda$ & $55-65$ \\
\hline [34] & $\begin{array}{c}\text { Implementing } \\
\text { magneto- } \\
\text { electric } \\
\text { dipoles } \\
\end{array}$ & -11.6 & 16.55 & $8 \times \lambda$ & $27-32$ \\
\hline [160] & $\begin{array}{l}\text { Implementing } \\
\text { arrays of E- } \\
\text { shape slots }\end{array}$ & -10 & 10.1 & $10 \times \lambda$ & $55-65$ \\
\hline [161] & $\begin{array}{l}\text { Using E- } \\
\text { shape arms }\end{array}$ & -4 & 8 & $0.059 \times \lambda$ & $0.93-3.65$ \\
\hline [162] & $\begin{array}{c}\text { Implementing } \\
\text { d-shape slots } \\
\text { with helix- } \\
\text { shape } \\
\text { inductor } \\
\end{array}$ & -1.5 & 3.5 & $0.083 \times \lambda$ & $1-4.2$ \\
\hline [163] & $\begin{array}{c}\text { Implementing } \\
\text { T- and } \Pi- \\
\text { shape slots }\end{array}$ & -11 & 7.8 & $3.43 \times \lambda$ & $57-62$ \\
\hline [164] & $\begin{array}{c}\text { Using } \\
\text { interdigital } \\
\text { slots }\end{array}$ & -2 & 5.12 & $0.375 \times \lambda$ & $0.7-8$ \\
\hline [165] & $\begin{array}{l}\text { Tapering } \\
\text { patch }\end{array}$ & -3 & 2.35 & $0.235 \times \lambda$ & $0.115-2.9$ \\
\hline
\end{tabular}

The reported peak realized gains in [34], [52]-[55], [71], [160], [161], and [163] are higher than achieved here. The smaller length of the proposed antenna compared to [52] could account for the differences. Moreover, the medium gain of our proposed antenna is 
due to the high conductor loss, which is the result of the realization on a thin substrate to achieve a low-profile antenna. Increasing thickness, number of slots, and antenna length result in higher gain at the expense of increasing the antenna's size.

The proposed LWA operates at a higher frequency band compared to [52]-[55], and [161], [162], [164], and [165]. Furthermore, [34], [53], [71], and [160]-[165] are different types of antennas using different radiation mechanisms compared to the proposed SIW LWA here, resulting in different SLL and gain. For example, [160]-[165] are metamaterial-based antennas in which radiation occurs from metamaterial cells. While [71] is an HMSIW-based antenna wherein wave leaks to space from the side aperture and interdigital slots. In addition, [53] is an air-filled waveguide-based LWA fed by through-hole connectors, and [34] is an array of magneto-electric dipoles.

\subsection{Conclusion}

In this chapter, two new approaches for reducing the SLL of LWA were proposed. The proposed antennas offered frequency beam-scanning with medium gain and low SLL.

The first method was based on modifying the aperture of an HMSIW LWA, resulted in the SLL of $-13.8 \mathrm{~dB}$ and $-11.2 \mathrm{~dB}$ in the upper hemisphere and full space, respectively. The reduction of SLL in all space is the key benefit of the proposed design method. In addition, the frequency beam-squint was reduced by tapering the aperture. However, the sensitivity of the SLL to the tapered aperture size is challenging. In other words, the SLL can degrade by small fabrication errors in tapering the side aperture. The proposed idea was realized on a $0.5 \mathrm{~mm}$ Rogers Duroid 5880 substrate. The fabricated HMSIW LWA scans space near broadside with $10.6 \mathrm{dBi}$ of gain and $-11.2 \mathrm{~dB}$ SLL over the frequency 
band of $26-30 \mathrm{GHz}$, which is useful as seeker antenna. A good agreement between the measurements and simulated results was observed. The discrepancies between measurement and simulation results were investigated by a thorough sensitivity analysis that includes the fabrication tolerances. The medium gain, low SLL, compactness, small frequency beam-squint, and simple fabrication process are among the advantages of the proposed HMSIW LWA, making it a suitable candidate for $5 \mathrm{G}$ point to point wireless communication systems and as seeker antenna in guiding systems.

The second method focused on the reduction of SLL of SIW LWA. Generally, the SLL of SIW-based LWA can be reduced by tapering the radiation and the feed section. Unfortunately, the effects of feed on radiation patterns were mostly ignored in the literature. Modifying the feed transition by adding tapered CPWs resulted in the SLL reduction of SIW LWA. Applying the proposed design, an improvement of about $7 \mathrm{~dB}$ in SLL compared to the conventional SIW LWA was achieved. The $10 \mathrm{~dB}$ impedance bandwidth of the fabricated SIW LWA is from $26 \mathrm{GHz}$ to $30 \mathrm{GHz}$. The measured SLL and peak realized gain are $-11.4 \mathrm{~dB}$ and $6.1 \mathrm{dBi}$, respectively. The low-profile, moderate gain, low SLL, and simplicity of the design are among the features which make the proposed SIW LWA a suitable candidate for 5G applications. The proposed SIW LWA radiates near the endfire with a $16^{\circ}$ beam-scanning range, which is a suitable antenna for radar systems to detect targets near the horizon. Moreover, it can be mounted on the vehicle bumper to monitor the blind spots.

A new method for enhancing gain of a compact LWA is investigated in Chapter 4. 


\section{Chapter 4: Gain Enhancement of Compact HMSIW LWAs}

One of the most important characteristics of an antenna is its gain. In wireless networks, implementing a medium to high gain antenna is required to compensate for the large path loss. Increasing the gain is also beneficial in terms of lower transmit power requirements. Gain can be increased by improving directivity or radiation efficiency. Directivity is usually enhanced by increasing the length, which is not always possible in communications devices. In this chapter, introducing cavities in the LTCC-based HMSIW LWA leads to an increase in radiation efficiency and gain. The proposed approach is similar to tapering the thickness of the substrate.

\subsection{Background}

LWAs are among medium to high gain antennas [28]-[31]. However, the gain of the antenna may be reduced as a consequence of enhancing other parameters such as SLL. This is due to the tradeoff between gain, return loss, and SLL. Hence, implementing a method for increasing the gain of the LWA is crucial. In this chapter, the gain of the LWA is improved by adding cavities into the structures.

It is difficult to taper the thickness of the substrate using PCB or LTCC technology. Moreover, implementing cavities in PCB structures requires multiple conductor layers that increase the conductor loss. In contrast, LTCC contains a stack-up of tape layers with no requirement of sandwiching by conductive layers. Hence, cavities are created by placing different tape layers on top of each other. Embedding cavities inside the LTCC structure is similar to tapering the thickness of the substrate. 
As stated in Chapter 2, the packaging tolerance of LTCC structure is higher than PCB since all layers in LTCC are fabricated simultaneously. Hence, LTCC technology is more suitable for highly dense integrated applications. However, surface roughness and thermal expansion of the tape layers are major issues in the LTCC design. As stated before, in an LTCC structure, silver or gold is used as conductive material and FerroA6M or Dupont9K7 is chosen as the tape layer due to their low loss.

\subsection{Antenna Structure}

The proposed HMSIW LWA was designed based on an LTCC structure containing two conductor layers. The top conductor plane carries the mm-wave signal and the bottom one is the ground plane of the antenna. Silver was chosen as the conductor material due to its excellent characteristics such as high conductivity and robustness to the environmental variation. As stated before, to prevent the structure from breaking and bending, the ground plane was meshed such that at least $50 \%$ of the conductive material was removed. The proposed antenna contains four dielectric tape layers. Ferro A6M with $\varepsilon_{r}=5.6, \mu_{r}=1$, and a loss tangent of 0.001 was chosen as the dielectric tape layer. The thickness of each tape layer was chosen as $0.127 \mathrm{~mm}$ to achieve a low-profile structure. However, the tape layer shrunk due to the baking in the fabrication process. The postfired thickness of each tape layer was $0.115 \mathrm{~mm}$. A dummy tape layer with a thickness of $0.5 \mathrm{~mm}$ underneath the ground plane was added to improve the mechanical strength. Doing so also prevented the antenna from bending excessively due to the baking.

The schematic view of the proposed LWA and the fabricated cavity-based LTCC LWA are demonstrated in Fig. 4.1. The length, width, and thickness of the antenna are 40 $\mathrm{mm}, 16 \mathrm{~mm}$, and $1 \mathrm{~mm}$, respectively. Assuming single-mode propagation of the 
fundamental HMSIW mode $\left(\mathrm{TE}_{0.5,0}\right)$ in the proposed antenna, the effective width of the HMSIW ( $w$ ) was calculated by (3.1). Assuming $f_{c}=27 \mathrm{GHz}$ and $\varepsilon_{r}=5.68$, the corresponding $w$ is $1.16 \mathrm{~mm}$. The center frequency, $\Delta \theta$, and $\theta_{0}$ were chosen as $28.5 \mathrm{GHz}$, $30^{\circ}$, and $70^{\circ}$, respectively, resulting in radiation length $L_{r}=22 \mathrm{~mm}$ according to (2.4). The length of the antenna's feed at each end was optimized to achieve the minimum return loss, which resulted in antenna's total length of $40 \mathrm{~mm}$. It should be noted that in (3.1) and (2.4) the effects of the embedded cavities were ignored. In other words, implementing the embedded cavities led to the deviations of $\Delta \theta$ and $\theta_{0}$ from the chosen values. Some of the geometrical parameters of the proposed LTCC LWA are reported in Table 4.1. Following the idea presented in Section 3.2, the side aperture was tapered in a trapezoid shape to improve the SLL, as shown in Fig. 4.1(b). The impedance matching was improved by implementing a tapered via fence in addition to the tapered microstrip transitions. Two surface-mounted $2.9 \mathrm{~mm}$ connectors were used at both ends of the LTCC LWA. The input connector was connected to a signal generator, while the output was connected to a matched load to absorb the un-radiated power.

The size of the cavities $\left(L_{c}\right.$ and $\left.W_{c}\right)$ and their locations $\left(z_{c}\right.$ and $\left.x_{c}\right)$ were optimized through full-wave simulations combining with the physical intuition to improve the peak realized gain. The parametric studies on the peak realized gain for several cavities' dimensions and locations are reported in Fig. 4.2. It should be noted that the width of the cavities $\left(W_{c}\right)$ was chosen as $6 \mathrm{~mm}$, which was about half of the guided wavelength $\left(\lambda_{\mathrm{g}} / 2\right)$ and resulted in resonator cavities. Embedding cavities into the antenna is similar to loading it with different air-based stubs. Therefore, changing the size and location of the cavities is similar to changing the stubs' locations and sizes, which disturb the field 
distribution and propagation constant of the leaky-wave. Henceforth, peak realized gain varies by changing $L_{c}, W_{c}, z_{c}$, and $x_{c}$.

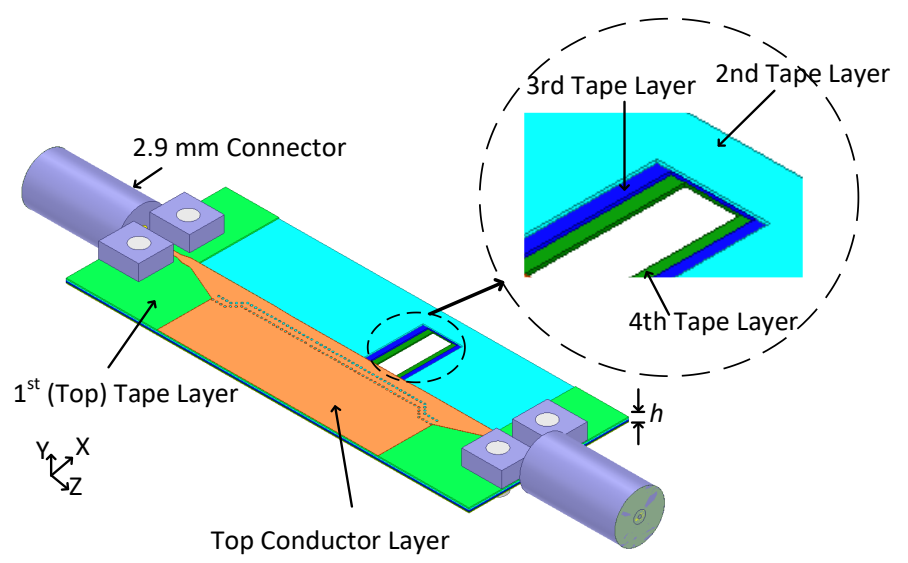

(a)

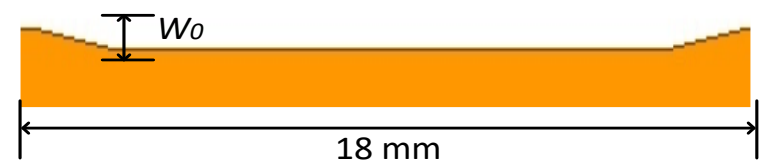

(b)

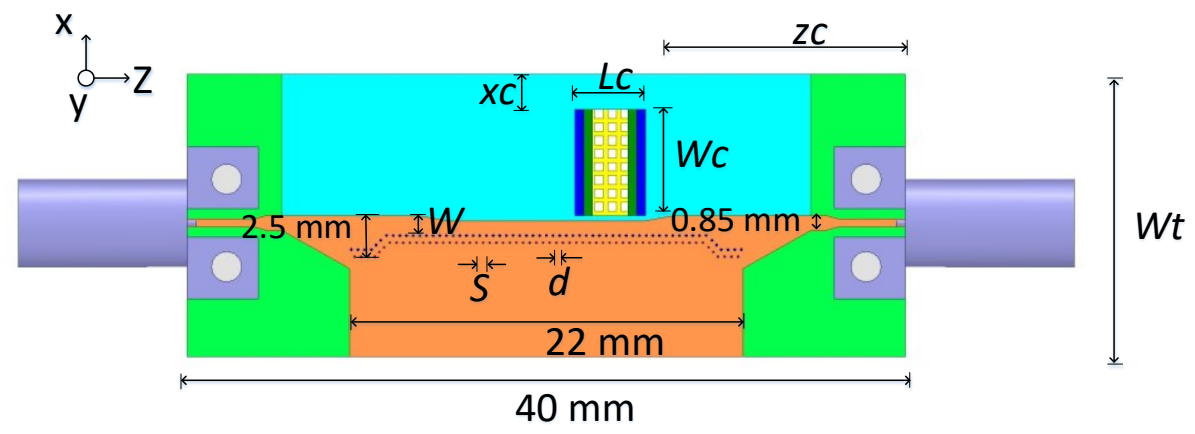

(c)

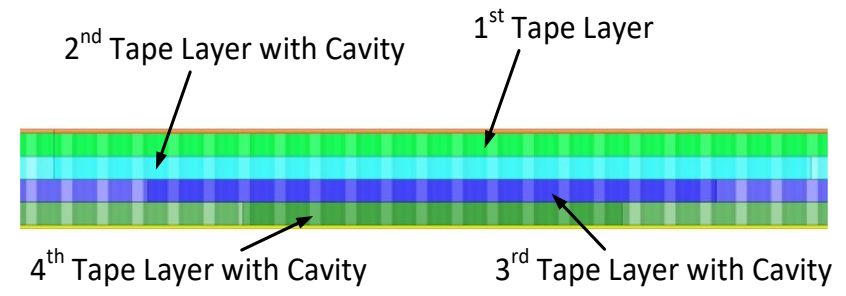

(d)

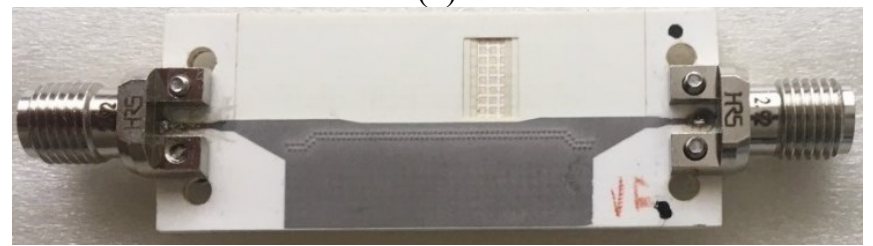

(e)

Figure 4.1. Schematic view of the proposed LTCC HMSIW LWA. (a) 3D view, (b) exaggerated view of the tapered aperture (non-scaled), (c) top view, (d) side view, and (e) fabricated antenna [87]. 
Table 4.1. Some of the geometrical parameters of the proposed LTCC antenna [87].

\begin{tabular}{|l|l|l|l|l|l|l|l|l|l|l|}
\hline Parameter & $h$ & $W$ & $W_{t}$ & $W_{c}$ & $L_{c}$ & $S$ & $d$ & $W_{0}$ & $x_{c}$ & $z_{c}$ \\
\hline Value (mm) & 1 & 1.16 & 16 & 6 & 4 & 0.38 & 0.14 & 0.3 & 2 & 14.6 \\
\hline
\end{tabular}
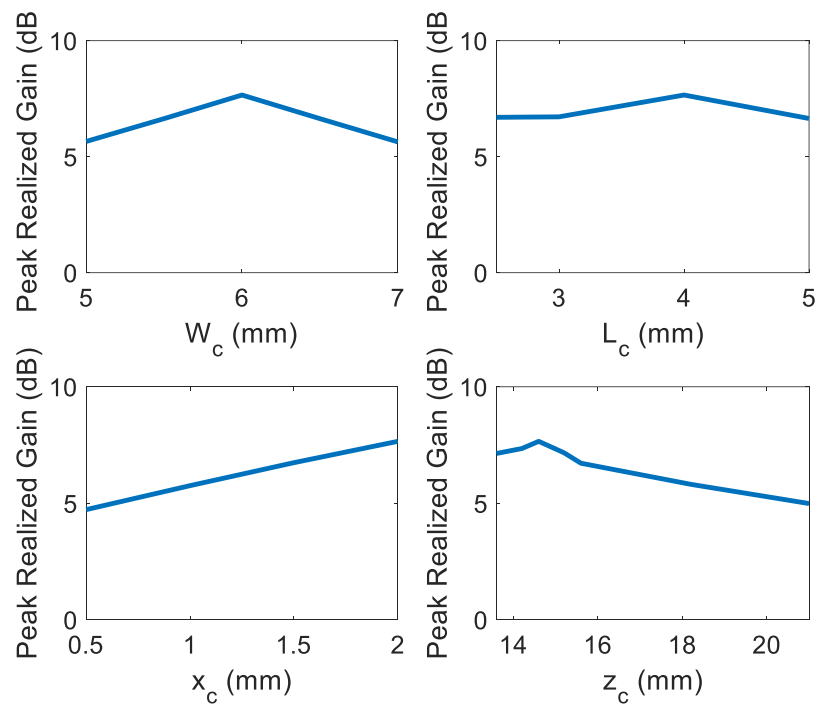

Figure 4.2. Peak realized gain of the proposed LTCC LWA for different sizes of cavities $\left(L_{c}\right.$ and $\left.W_{c}\right)$ and their locations $\left(x_{c}\right.$ and $\left.z_{c}\right)$ at $28.5 \mathrm{GHz}[87]$.

Due to fabrication technology limitations, it is not possible to place a cavity under the conductor layer. Hence, all cavities were realized in the uncovered sections of the dielectric tape layers. This imposes restrictions on the realization of the proposed design procedure to improve gain. As stated before, the proposed idea can also be implemented in a PCB structure. However, the packaging tolerance of the LTCC structure is better than the multi-layer PCB structure.

To prove the advantages of the proposed LWA, a multi-layer conventional non-loaded LTCC LWA and a tapered height LTCC LWA were investigated. The tapered height and the conventional LTCC LWAs are illustrated in Fig. 4.3. Our proposed cavity-based LTCC LWA consists of step-shape cavities, while the tapered height LTCC antenna contains a clean tapered cavity in the tape layer (shown in blue). In the tapered height LTCC LWA, the cavity's walls were tapered to emulate the angle $\delta$ from the horizon. In 
other words, if we consider the cavity as a horn aperture in the $\mathrm{XZ}$ plane, $\delta$ is the flare angle. Therefore, varying $\delta$ changes the cavity size, which affects the propagation of the leaky-wave and the peak realized gain.

\subsection{Simulated and Measured Results}

Assuming the fundamental mode propagates in the $z$-direction, the aperture electric field components are determined by (3.2)-(3.6) [138]. According to (3.5) and (3.6), changing $h$ leads to variations in $k_{y}$ and $k_{z}$ affecting the aperture fields. The far-zone electromagnetic fields are obtained by taking the fast Fourier transform of the aperture fields using the well-known equations reported in [138]. Overall, introducing the cavities, which resembles tapering the thickness $(h)$, causes variations in the radiation pattern.

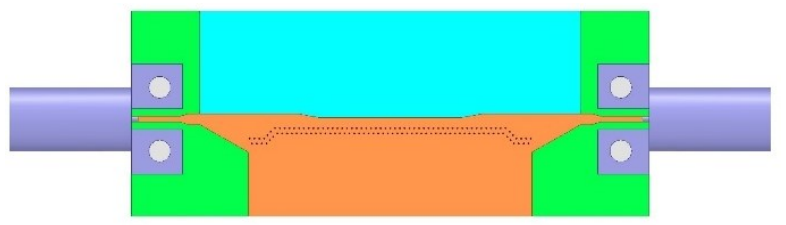

(a)

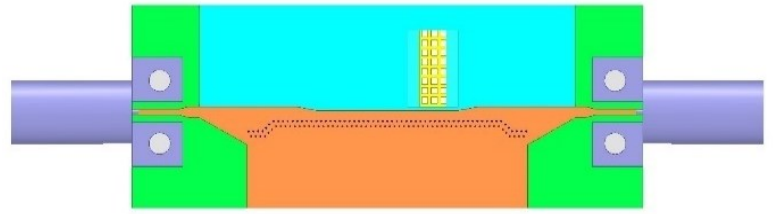

(b)

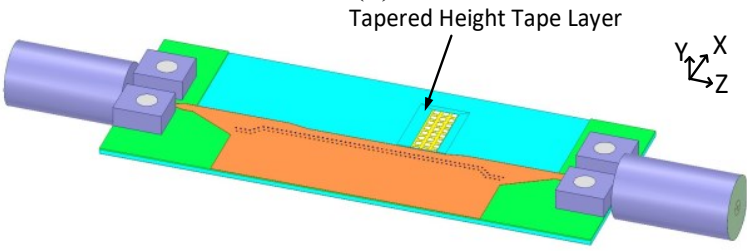

(c)

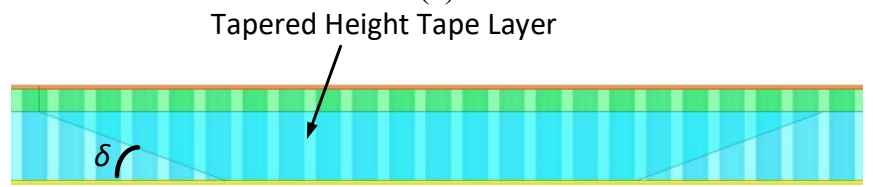

(d)

Figure 4.3. Schematic view of the multi-layer conventional LTCC LWA and tapered height LTCC antenna [87]. (a) top view of the conventional non-loaded LTCC LWA, (b) top view of the tapered height LWA, (c) 3D view of the tapered height LWA, and (d) side view of the tapered height antenna. 
Preliminary simulations have been carried out using Ansys HFSS v.19. As stated before in Chapter 3, the S-parameters were measured using a Rohde \& Schwarz ZVA-67 VNA. The S-parameter diagrams are shown in Fig. 4.4. According to Fig. 4.4, the proposed structure demonstrates a return loss better than $10 \mathrm{~dB}$ from $28.3 \mathrm{GHz}$ to 29 GHz. It should be noted that the proposed antenna was optimized to improve gain rather than the impedance bandwidth. In other words, the relatively small impedance bandwidth (i.e., $700 \mathrm{MHz}$ ) is due to the trade-off between gain and the return loss. The corresponding insertion loss ranges from $10 \mathrm{~dB}$ to $15 \mathrm{~dB}$ representing the low loss.

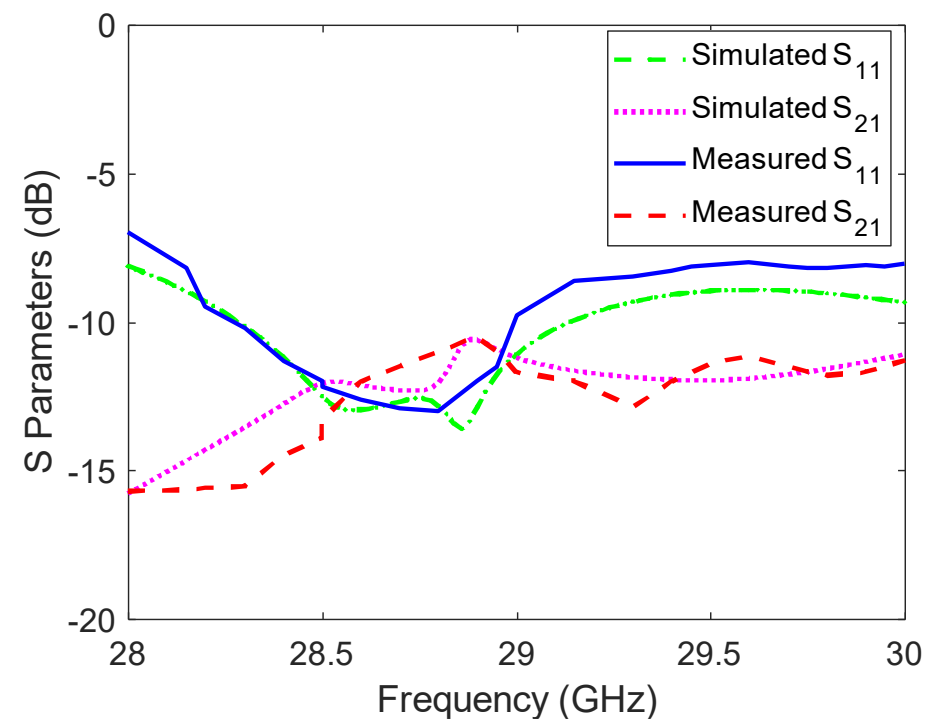

Figure 4.4. Measured and simulated S-parameters of the proposed LTCC based antenna.

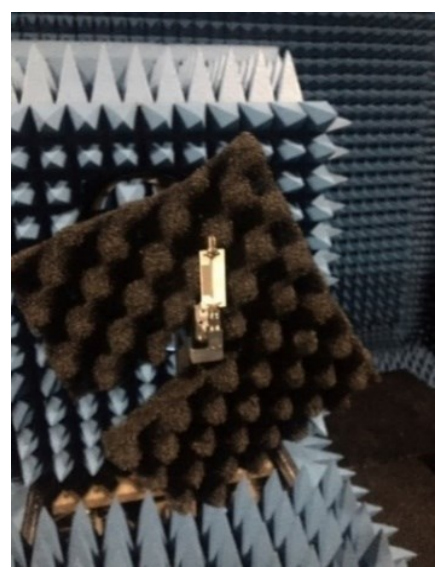

(a)

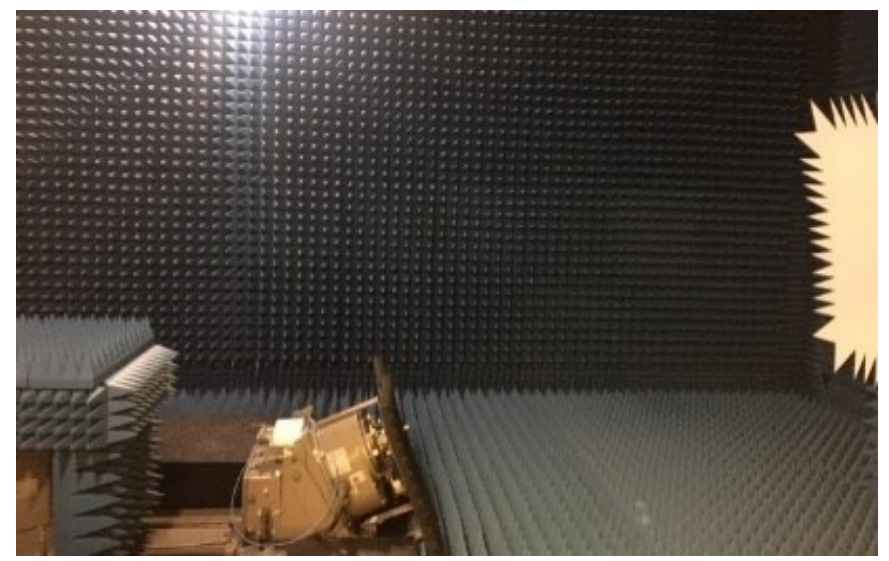

(b)

Figure 4.5. Measurement setup of the radiation pattern. (a) the AUT, (b) the anechoic chamber [87]. 
The radiation pattern was measured in the anechoic chamber using the MI-12A-26 reference gain horn antenna similar to the previous measurement setups, as presented in Fig. 4.5. The measured and simulated radiation patterns of the LTCC LWA at $28.5 \mathrm{GHz}$ are presented in Fig. 4.6. It should be noted that the reported radiation patterns are the realized gains that take the mismatch into account. The possible shrinkage and bending of the dielectric and conductive layers due to baking are among the main reasons for discrepancies between measurements and simulated results.

To show the gain improvement more vividly, the radiation pattern of the multi-layer conventional non-loaded LTCC LWA is reported in Fig. 4.6 as well. As observed in Fig. 4.6, embedding cavities increases the gain. Moreover, implementing cavities leads to variations in $\Delta \theta$ and $\theta_{0}$. The measured main-beam radiates at $\theta_{0}=66^{\circ}$. As stated in Chapter 3, the reported radiation patterns correspond to the E-plane (YZ-plane) because measuring the H-plane is out of the capability of the measurement system.

Radiation patterns at $28.3 \mathrm{GHz}$ and $28.7 \mathrm{GHz}$ are presented in Fig. 4.7. Because of the leaky-wave nature of the proposed LWA, the beam steers by sweeping the frequency [28]-[30]. Tapering the side aperture and introducing cavities lead to the reduction of the frequency sensitivity i.e., smaller beam-scanning range by sweeping frequency. This is due to the decrease in the effective dielectric constant, which leads to minor variations in the phase constant by sweeping the frequency. It is appealing for applications in which frequency beam squint is not desirable, such as seeker antenna or point-to-point communications. According to Fig. 4.7, the proposed antenna posed a $3^{\circ}$ beam squint in the frequency band of 28.3-28.7 GHz. Furthermore, sweeping the frequency leads to variations in the attenuation constant [28]-[30], resulting in the variations in SLL. 


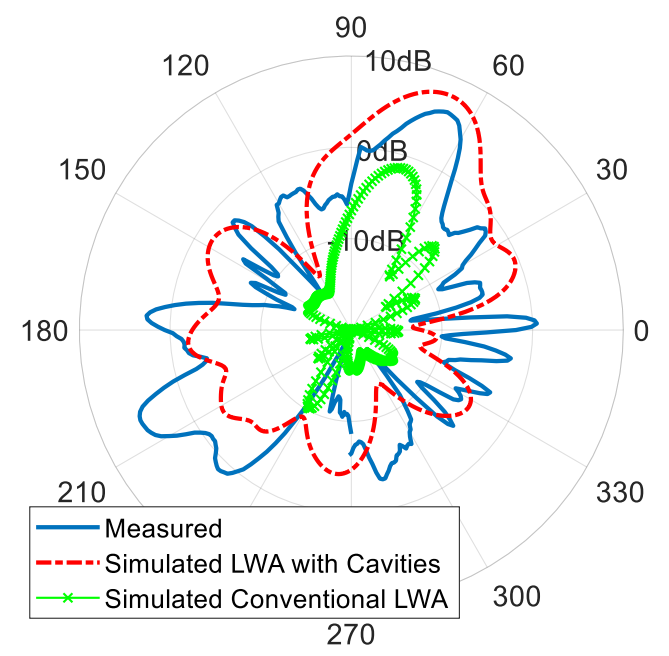

Figure 4.6. Measured and simulated radiation patterns of the proposed LTCC LWA with embedded cavities and the multi-layer conventional non-loaded LTCC LWA at $28.5 \mathrm{GHz}$ and E-plane [87].

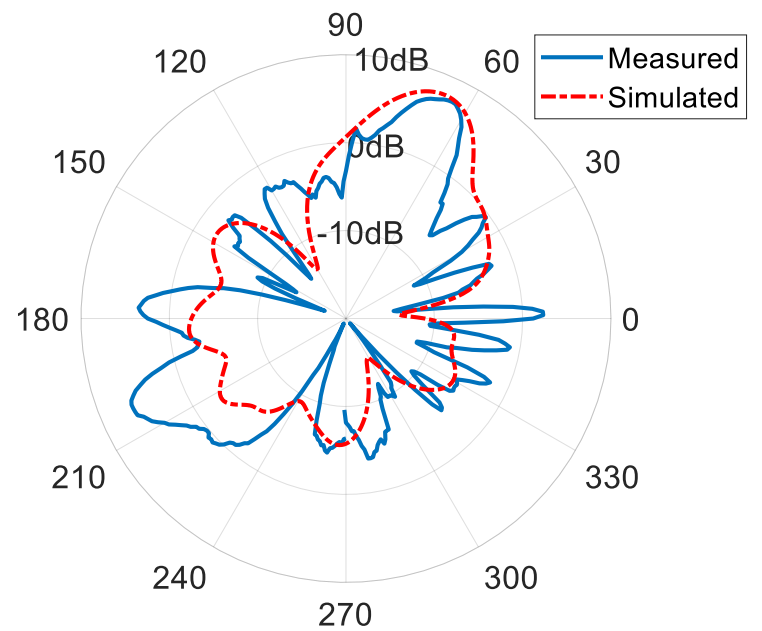

(a)

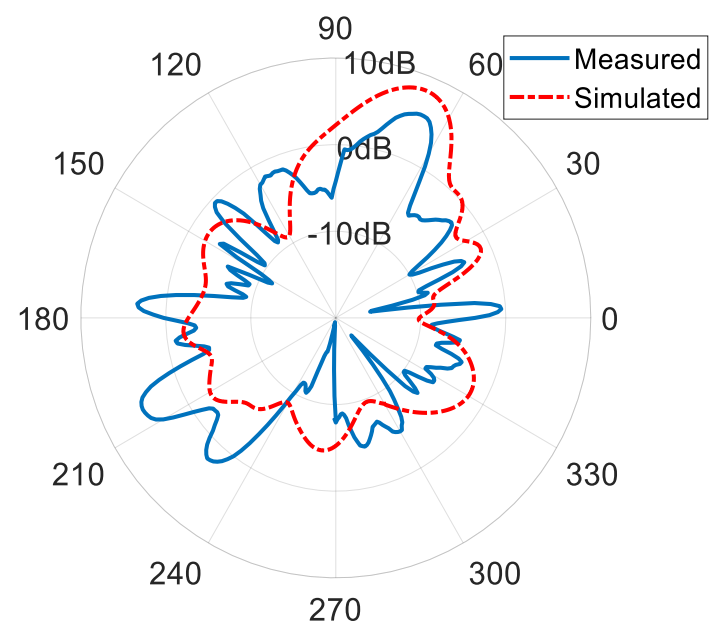

(b)

Figure 4.7. Gain radiation patterns diagrams of the proposed LTCC LWA with embedded cavities at different frequencies [87]. (a) $28.3 \mathrm{GHz}$, (b) $28.7 \mathrm{GHz}$.

The measured and simulated peak realized gain of the proposed antenna with and without cavities are presented in Fig. 4.8, confirming that introducing the cavities increases the peak realized gain of the antenna. This is because introducing cavities into the structure is the same as tapering the thickness of the structures, which causes the field perturbation. It should be noted that gain improvement is mostly due to the increase of the radiation efficiency rather than the directivity. The HPBW remains almost constant 
by embedding the cavities, as observed in Fig. 4.6. Therefore, directivity does not change a lot by integrating the cavities into the antenna. This indicates that gain improvement is mostly because of the rise of the radiation efficiency rather than the directivity. In the proposed cavity-based LTCC LWA and the conventional non-loaded LTCC LWA, the thinnest Ferro A6M tape layer was chosen to achieve a low-profile structure. This led to high conduction loss that explains the small peak realized gain of the conventional nonloaded LTCC LWA, while the gain of the cavity-based LTCC LWA was improved due to the presence of the cavities.

The simulated peak realized gain of the tapered height LTCC LWA for different values of $\delta$ is shown in Fig. 4.9. As observed in Figs. 4.8 and 4.9, both embedding cavities and tapering the height improve the gain. However, tapering the thickness smoothly is not possible due to the fabrication technology limitation. Therefore, embedding the cavities was chosen as the method for improving the gain. As observed in Figs. 4.1(d) and 4.3(d), the chosen dimensions of the embedded cavities are mapped to tapered thickness with $\delta=20^{\circ}$, corresponding to the maximum peak realized gain. Implementing the proposed design on a thicker substrate should result in higher gain [63]. This is achieved at the expense of increasing the size of the antenna. Since the proposed LTCC LWA was optimized to improve the gain, the return loss and SLL were not optimum. As mentioned in Chapter 3, the high SLL of an HMSIW-based antenna is mainly due to the ground plane's truncation and reflection from the feed and the aperture. As stated before, tapering the via fence and the aperture are among the methods for reducing the SLL of HMSIW LWA. 


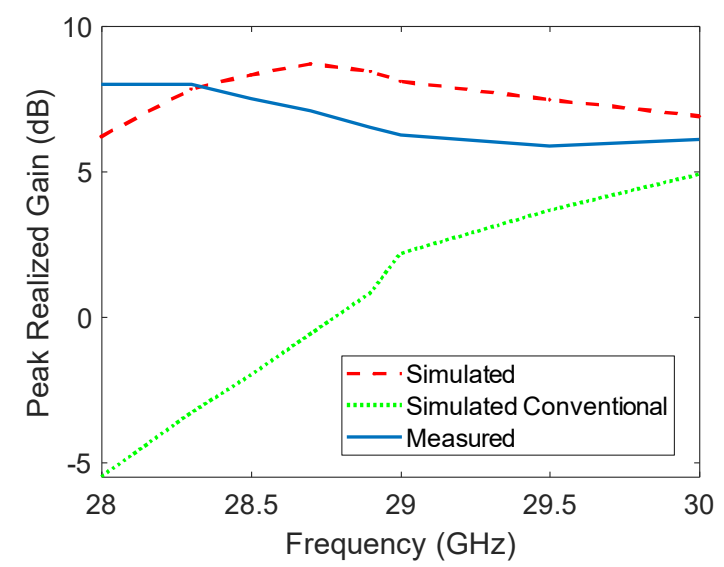

Figure 4.8. Peak realized gain diagrams of the proposed LTCC HMSIW LWA [87].

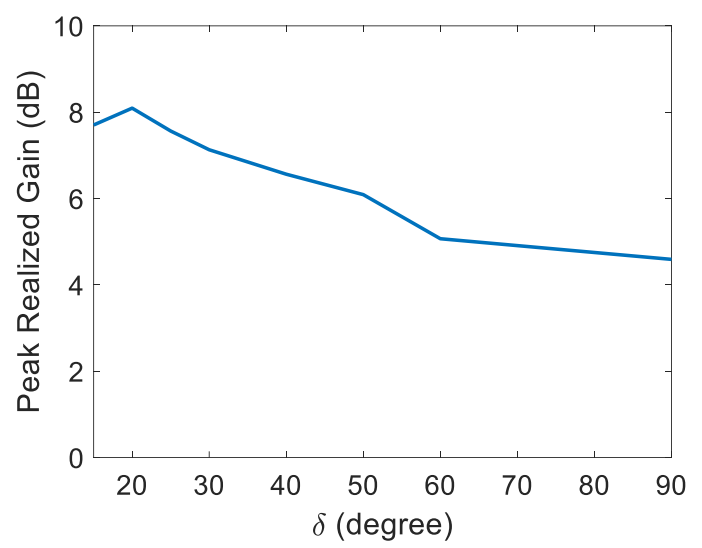

Figure 4.9. Simulated peak realized gain of the tapered height LTCC LWA at $28.5 \mathrm{GHz}$ [87].

A good agreement between the measured and the simulated results was observed. Expansion of the conducting and tape layers and deviations in the via fence locations contribute the most to the discrepancies. Moreover, the possible air gaps between the tape layers [93] leads to discrepancies. The surface roughness and bumpiness of the layers can also result in discrepancies between measured and simulated results.

A detailed parametric study was carried out to investigate the discrepancies between simulated and measured results. To do so, different parameters such as the thickness of the substrate $(h)$, via size $(d)$, via spacing $(S)$, the waveguide section's width $(W)$, and the cavities sizes ( $W_{c}$ and $L_{c}$ ) were changed. It should be noted that only one parameter was changed in each case, while the others stated in Table 4.1 were held constant. The Sparameters for different parameters are reported in Fig. 4.10. According to Fig. 4.10, W, 
$d$, and $S$ have the most dominant effects on the S-parameters. This is because $W, d$, and $S$ determine the field confinement and cut-off frequency of the dominant mode. The normalized radiation patterns for different parameters are illustrated in Fig. 4.11, indicating that $W, d$, and $S$ play the dominant role in the SLL and nulls' locations of the radiation pattern since these parameters determine the field confinement by the via fence. The peak realized gains of the antenna, considering different parameters, are reported in Fig. 4.12, demonstrating that changing $W$ leads to extreme variations in the peak realized gain. This is because changing $W$ leads to the variations in $f_{c}$ and propagation constant.

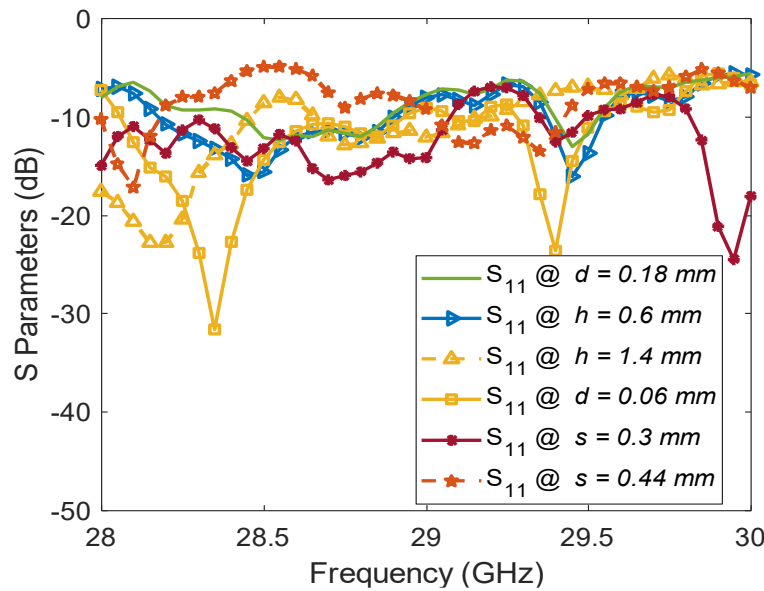

(a)

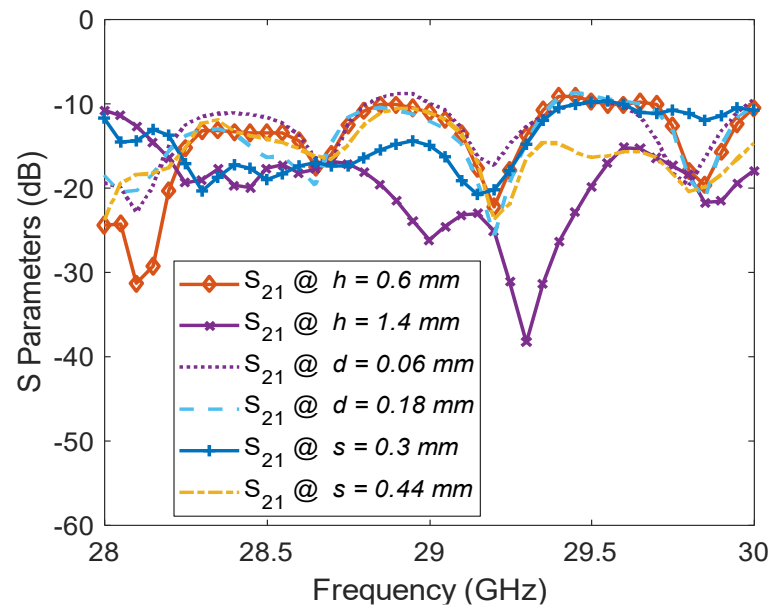

(c)

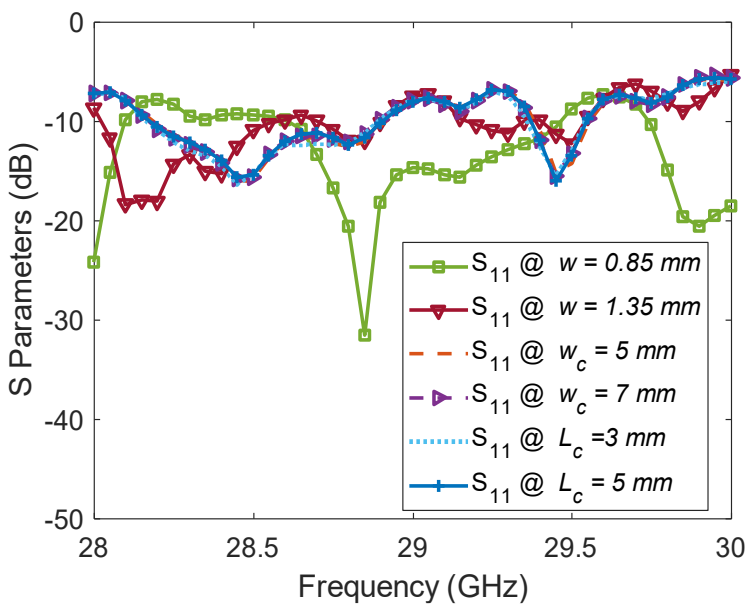

(b)

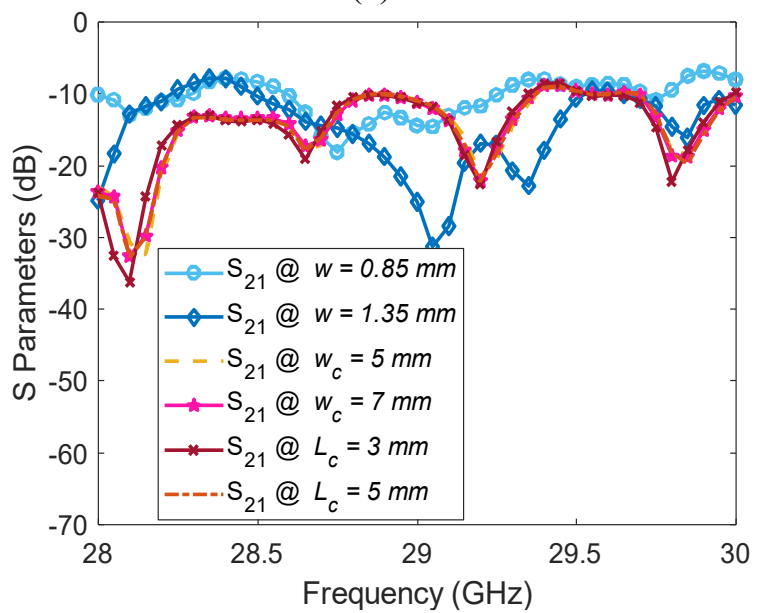

(d)

Figure 4.10. S-parameters of the proposed LTCC LWA with different parameters [87]. (a) S11 for different values of $h$, d, and s, (b) S11 for different values of W, Lc, and Wc. (c) S21 for different values of $h$, d, and s, (d) S21 for different values of W, Lc, and Wc. 


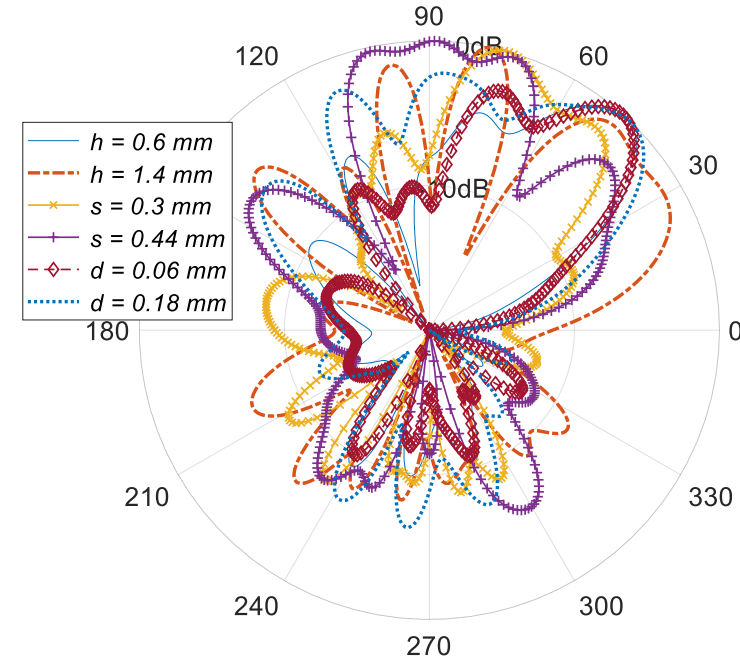

(a)

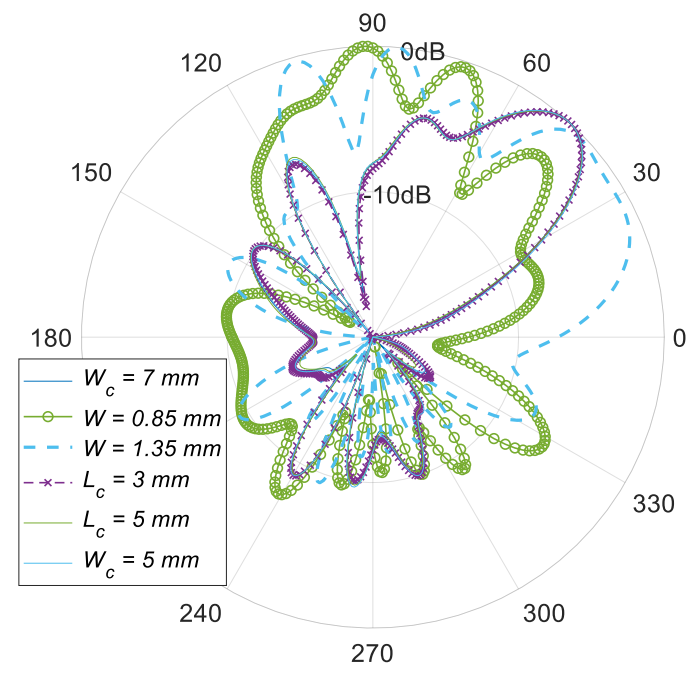

(b)

Figure 4.11. Normalized radiation patterns of the proposed LTCC antenna obtained from changing different parameters at $28.5 \mathrm{GHz}$ [87]. (a) different values of $h, d$, and $s$, (b) different values of $W, L_{c}$, and $W_{c}$.

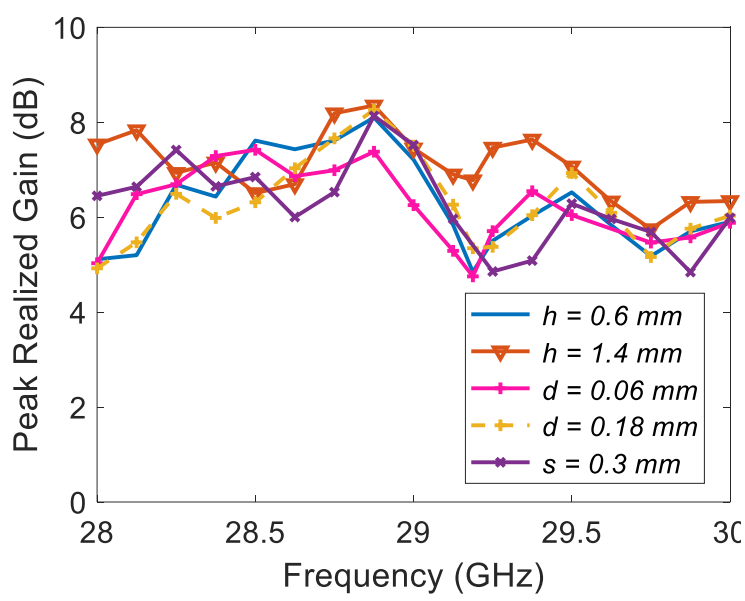

(a)

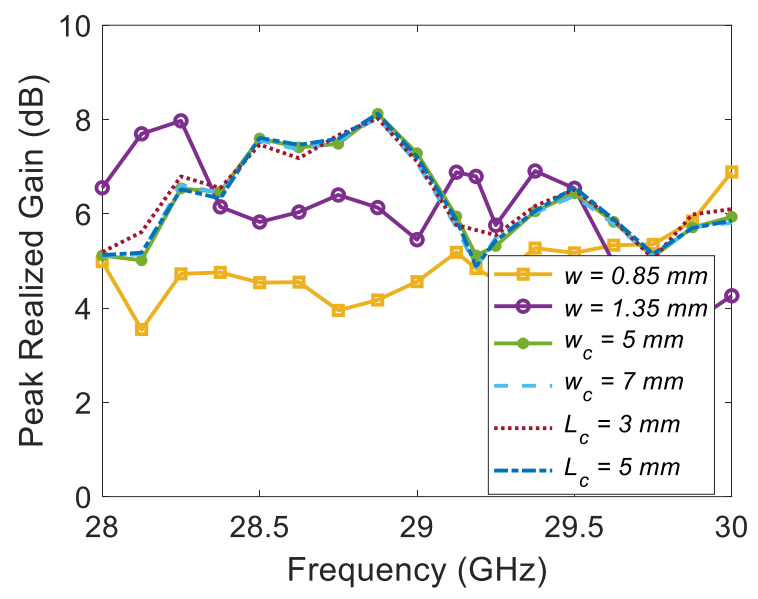

(b)

Figure 4.12. Peak realized gains of the LTCC antenna corresponding to different parameters [87]. (a) different values of $h, d$, and s, (b) different values of W, Lc, and Wc.

The size of the ground plane is an essential factor in the SLL and main-lobe pointing angle. To investigate the effect of the size of the ground plane, the proposed LTCC LWA with different width $\left(W_{t}\right)$ were simulated, while the other parameters stated in Table 4.1 were held constant. The corresponding S-parameters, normalized radiation patterns 
diagrams, and peak realized gain are reported in Figs. 4.13-4.15. Changing $W_{t}$ leads to variations in the width of the dielectric aperture and the ground plane affects the peak realized gain. Assuming this aperture as a horn antenna's aperture in the YZ plane, varying $W_{t}$ is similar to changing the length of the horn antenna without changing the flare angle. This changes the modal configuration of the structure, leading to the variations in $\beta, \theta_{0}$, and the peak realized gain. The optimum value of $W_{t}$ was obtained through full-wave simulation along with the physical understanding to achieve the maximum peak realized gain.

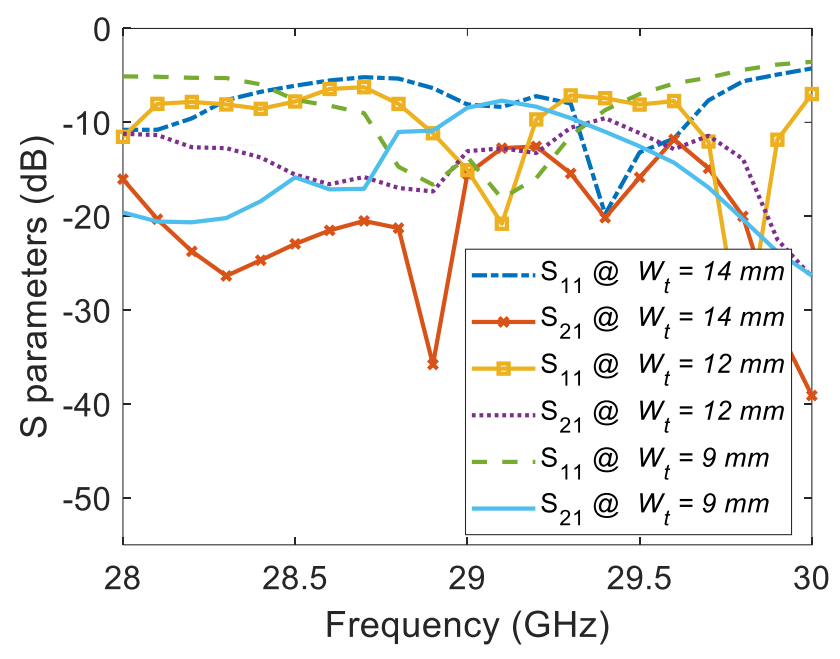

Figure 4.13. S-parameters of the proposed LTCC antenna with different Wt [87].

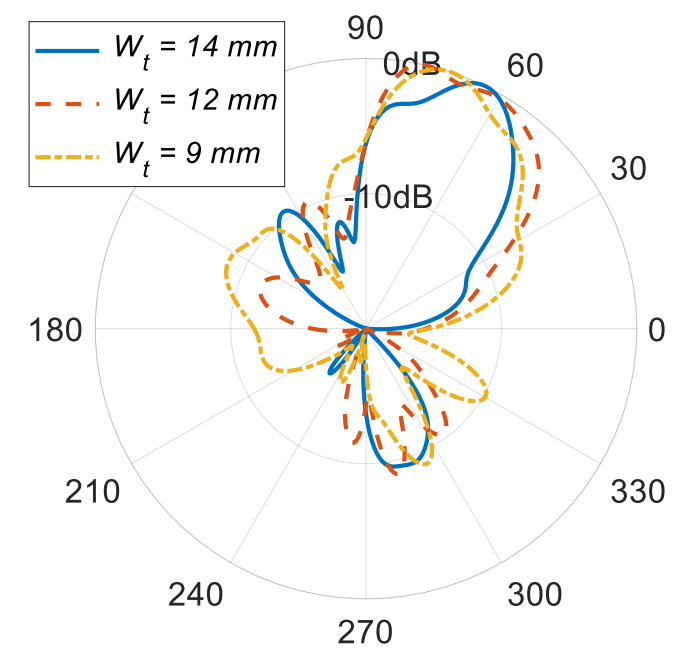

Figure 4.14. Normalized radiation patterns of the proposed LWA with different Wt at $28.5 \mathrm{GHz}$ [87]. 


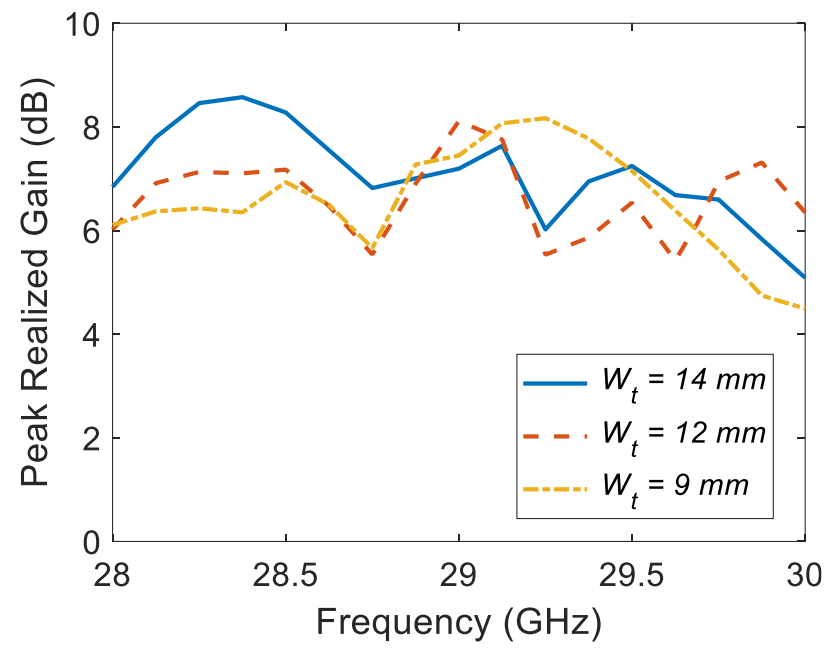

Figure 4.15. Peak realized gain diagrams of the LTCC antenna correspond to different $W_{t}$ [87].

As investigated in Chapter 3, placement of the surface mounted connectors on the antenna structure can bend the ends of the antenna due to the small thickness of the structure. Moreover, the connectors may not be accurately attached to the board due to assembly errors. To simulate such a case, the connector pin angle was offset from the horizon by angle $\xi$, as illustrated in Fig. 3.16. The corresponding S-parameters, normalized radiation patterns, and peak realized gain are reported in Figs. 4.16-4.18, respectively. According to Fig. 4.16, changing $\xi$ leads to a large mismatch. Fig. 4.17 indicates that offsetting the connectors increases the SLL and the lobe levels of the beams radiating beneath the antenna. According to Fig. 4.18, the peak realized gain degrades by changing $\xi$. Overall, rotating the connector causes discrepancies because the connector center pin is not touching the signal trace properly and only a small portion of the input signal transfers to the radiating section of the antenna. 


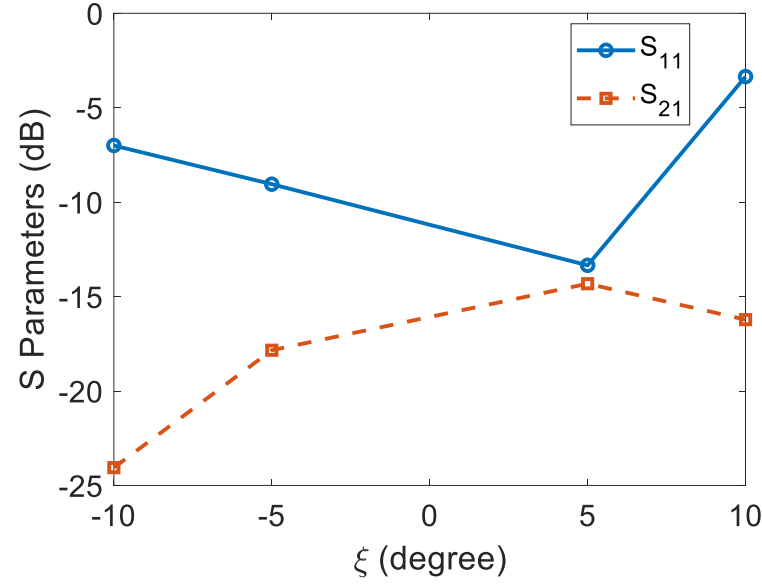

(a)

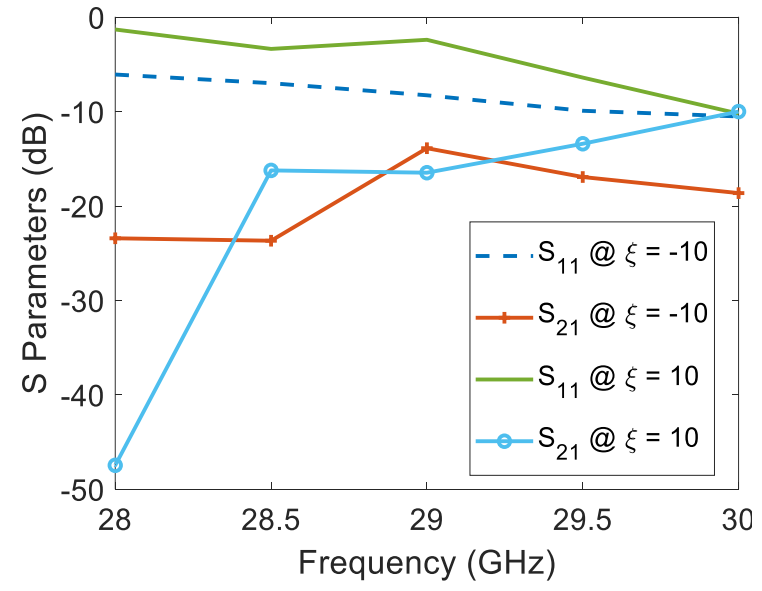

(b)

Figure 4.16. S-parameters of the proposed LTCC LWA with offset connectors [87]. (a) at $28.5 \mathrm{GHz}$

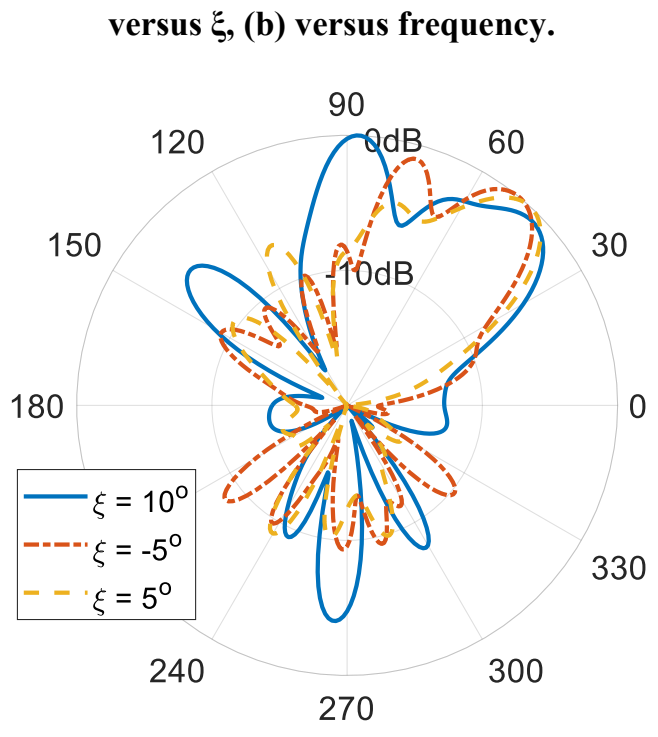

Figure 4.17. Normalized radiation patterns of the proposed HMSIW LTCC LWA with connectors offset from the horizon at $28.5 \mathrm{GHz}$ [87]. 


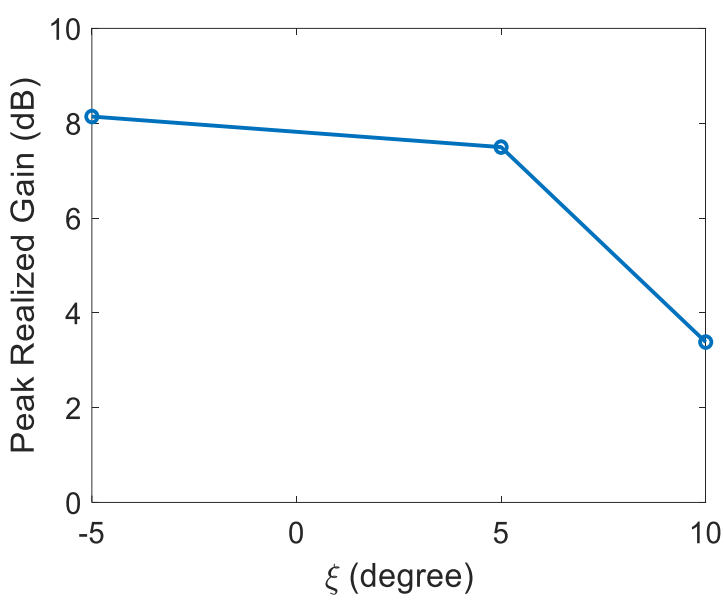

(a)

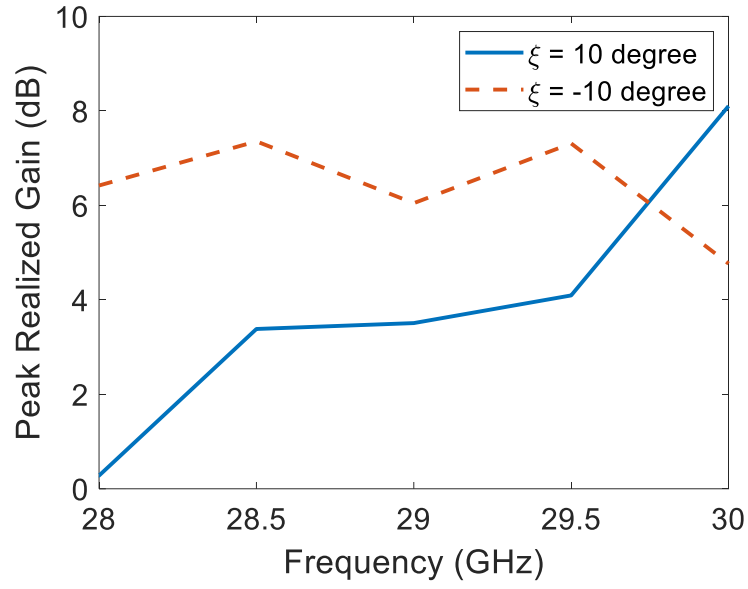

(b)

Figure 4.18. Peak realized gain of the proposed LTCC antenna with rotated connectors [87]. (a) at

$28.5 \mathrm{GHz}$ versus $\xi$, (b) versus frequency.

The antenna may bend due to the baking of the layers as part of the LTCC fabrication procedure. To investigate such case, the antenna was bent such to deviate gradually up to $h_{b}=6 \mathrm{~mm}$ from the horizon by moving toward the two connectors, as illustrated in Fig. 4.19. The S-parameters, normalized radiation patterns, and peak realized gain of the bent antenna are reported in Figs. 4.20-4.22, respectively. According to Figs. 4.20-4.22, bending the substrate leads to severe degradation of the antenna responses in terms of return loss, SLL, and gain. However, LTCC has high packaging tolerance, which minimizes the possibility of bending due to heating or physical pressure.

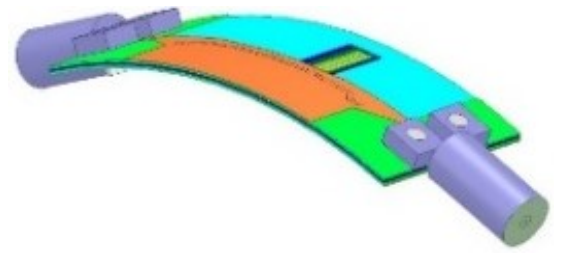

(a)

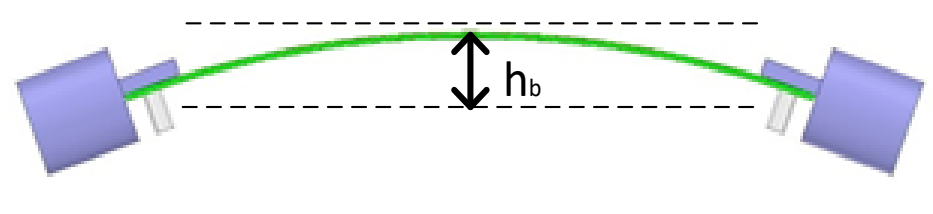

(b)

Figure 4.19. Schematic view of the bent LTCC antenna [87]. (a) 3D view, (b) side view. 


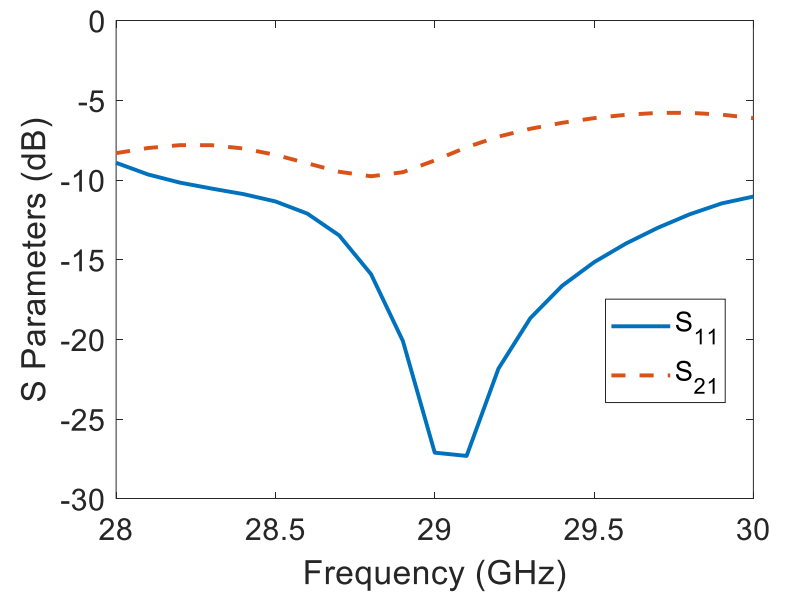

Figure 4.20. S-parameters of the proposed bent LTCC LWA [87].

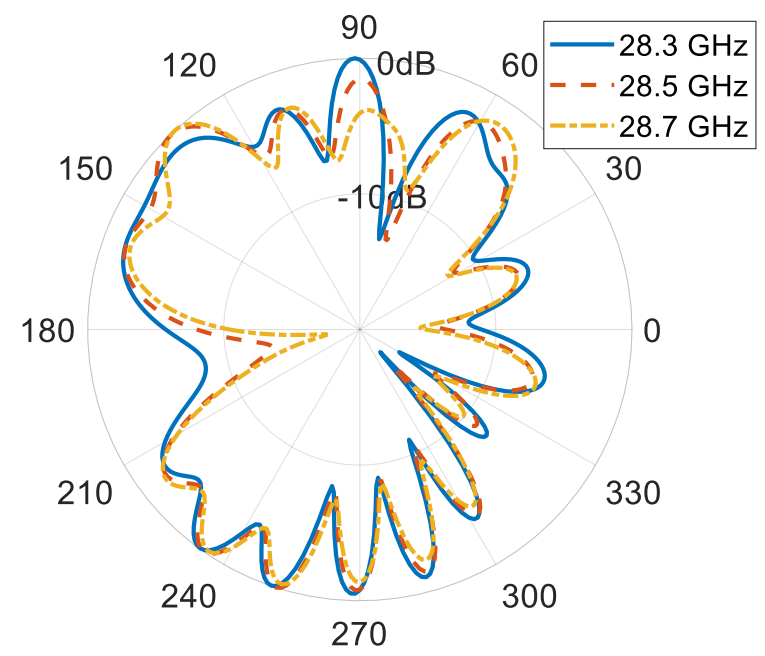

Figure 4.21. Normalized radiation patterns of the proposed bent HMSIW LTCC LWA [87].

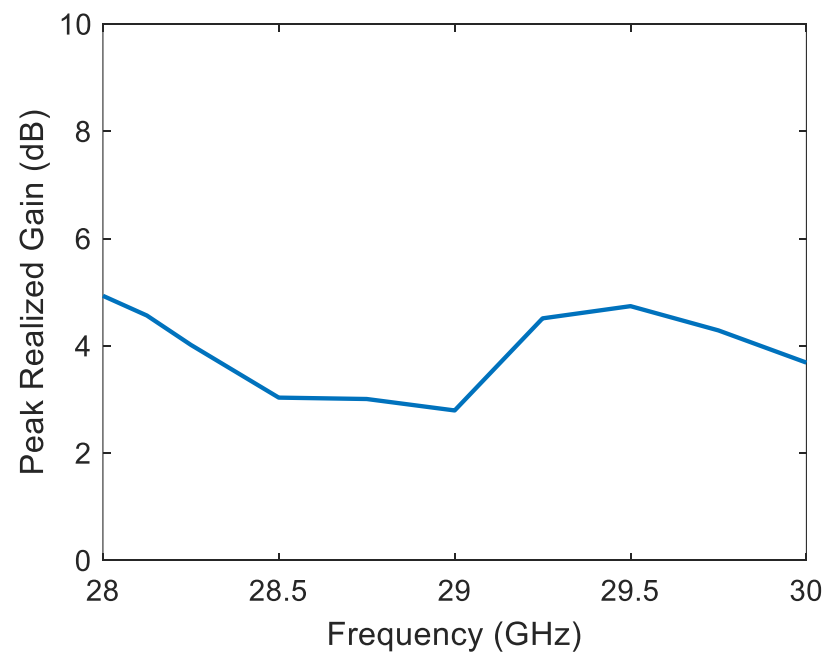

Figure 4.22. Peak realized gain of the proposed bent LTCC antenna [87]. 
Since the LTCC fabrication process involves heating the structure, deviations in via size and spacing, and total width $\left(d, S, W\right.$, and $\left.W_{t}\right)$ are quite likely. Meandering the board and deviation in the layer thickness can also occur due to the heating. Overall, the discrepancies between simulated and measured results are mostly due to the fabrication errors in $d, S, W$, and $W_{t}$, and errors in the assembly of the connectors.

To examine the advantages of the proposed design, a comparison with similar antennas is reported in Table 4.2. The peak realized gain of the proposed LTCC LWA here is smaller than the reported ones in [63], [65], [80], and [81]. This is because the length of the antennas in [63], [65], and [81] are longer than the corresponding value of the proposed LWA. The high gain of [80] was mainly due to the implementation of planar arrays of patch antennas. Moreover, the reported antenna in [81] is an LTCC horn antenna, which is realized by stacking up different tape layers. In [79], gain was increased by implementing metamaterial cells in the structure, which adds to the complexity and cost of the antenna. In contrast, embedding cavities in our proposed LTCC LWA does not increase the complexity and the cost of the fabrication. This comparison points out that the proposed LTCC LWA is a suitable candidate for $5 \mathrm{G}$ miniaturized applications.

\subsection{Conclusion}

The proposed LWA radiates near broadside with medium gain over the frequency bandwidth from $28.3 \mathrm{GHz}$ to $29 \mathrm{GHz}$, which is useful for point to point communications or radar seeker system. The main contribution of the proposed design in Chapter 4 is the gain improvement without increasing the size of the antenna and adding extra sections to the structure. Introducing cavities in the structure resulted in increasing gain. However, 
Table 4.2. Comparison of the proposed cavity-based LTCC LWA with similar designs [87].

\begin{tabular}{|c|c|c|c|c|}
\hline Reference & Methodology & $\begin{array}{l}\text { Peak Realized } \\
\text { Gain (dBi) }\end{array}$ & Antenna Length & $\begin{array}{c}\text { Frequency Band } \\
\text { (GHz) }\end{array}$ \\
\hline $\begin{array}{c}\text { Our proposed } \\
\text { cavity-based LWA }\end{array}$ & $\begin{array}{l}\text { Creating cavities in } \\
\text { the dielectric tapes }\end{array}$ & 7.6 & $4 \times \lambda$ & $28.3-29$ \\
\hline [65] & Tapering via fence & 15.6 & $8 \times \lambda$ & $6.5-10$ \\
\hline$[75]$ & Tapering via fence & 6 & $10 \times \lambda$ & $6.7-17$ \\
\hline [80] & $\begin{array}{l}\text { Implementing a } \\
\text { planar array of } \\
\text { patch antennas }\end{array}$ & 16 & $4 \times \lambda$ & $56-65$ \\
\hline [79] & $\begin{array}{c}\text { Implementing } \\
\text { metamaterial } \\
\text { structures }\end{array}$ & 6.93 & $0.2 \times \lambda$ & $5.25-5.45$ \\
\hline [63] & $\begin{array}{l}\text { Removing half of } \\
\text { the top cladding }\end{array}$ & 11.7 & $12 \times \lambda$ & $25-28$ \\
\hline [67] & $\begin{array}{l}\text { Implementing } \\
\text { different slots at } \\
\text { open aperture }\end{array}$ & 5.8 & $1.5 \times \lambda$ & $2.98-3.04$ \\
\hline [81] & $\begin{array}{l}\text { Stack-up tape } \\
\text { layers to form a } \\
\text { horn antenna }\end{array}$ & 18 & $12 \times \lambda$ & $230-330$ \\
\hline
\end{tabular}

fabrication technology limitations restricted the gain improvement capability. The peak realized gain of the fabricated antenna was $7.6 \mathrm{dBi}$ at $28.5 \mathrm{GHz}$. A good agreement between the measured and simulated results was observed. The compactness, high packaging tolerance, medium gain, low loss, and small frequency beam-squint of the proposed antenna make it a suitable choice for $5 \mathrm{G}$ miniaturized point to point communications and the seeker antenna.

A comparison among the proposed antennas in Chapters 3 and 4 is made in Table 4.3. The low SLL of the proposed antennas in Chapter 3 reduces the possibility of detection error and interference. Radiating with low SLL in the mm-wave frequency band, low- 
profile, and simplicity of designs are among the unique features of the proposed antennas in Chapter 3. The reported antenna in chapter 4 radiates with medium gain, which is suitable to compensate for some path loss. The proposed antenna in Chapter 4 is a suitable candidate for $5 \mathrm{G} \mathrm{mm}$-wave base station due to its appealing features such as compactness, medium gain, and robustness to environmental conditions.

The electronic beam-scanning capability of the LWA is achieved by implementing active elements. Several designs are investigated in chapter 5 to achieve electronic beamscanning.

Table 4.3. Comparison of the proposed antennas in Chapters 3 and 4.

\begin{tabular}{|c|c|c|c|c|}
\hline Antenna Type & Gain (dBi) & SLL (dB) & Antenna Length & Impedance Bandwidth \\
\hline $\begin{array}{c}\text { HMSIW LWA } \\
\text { with tapered } \\
\text { aperture }\end{array}$ & 10.6 & -11.2 & $6 \times \lambda$ & $26-30 \mathrm{GHz}$ \\
\hline $\begin{array}{c}\text { SLW LWA with } \\
\text { Tapered CPW feed }\end{array}$ & 6.1 & -11.4 & $10 \times \lambda$ & $26-30 \mathrm{GHz}$ \\
\hline $\begin{array}{c}\text { Cavity-based } \\
\text { HMSIW LTCC } \\
\text { LWA }\end{array}$ & 7.6 & -3 & $4 \times \lambda$ & $28.3-29 \mathrm{GHz}$ \\
\hline
\end{tabular}




\section{Chapter 5: Electronic Beam-Scanning in Low-Profile}

\section{LWAs}

A beam-scanning antenna is essential for dynamic communications links and navigation systems. There are generally three methods to achieve beam-scanning: frequency beam-scanning, mechanical beam-scanning, and electronic beam-scanning. Mechanical beam-scanning requires equipment to rotate the antenna physically, which is not suitable for compact devices that is out of the scope of this dissertation. Frequency beam-scanning is achieved by sweeping the frequency. Introducing sets of switches on the antenna and tuning bias voltages lead to electronic beam-scanning [94]-[133], which is the method followed here. The electronic beam-scanning antenna is also known as fixed frequency beam-scanning antenna or reconfigurable antenna. Different types of reconfigurable LWAs, such as HMSIW LWAs with circular reconfigurable cells and SIW LWAs with longitudinal reconfigurable cells, for 5G mm-wave beam-scanning are proposed in this chapter.

\subsection{General Background}

As stated in Chapter 2, deployment and maintenance of agile communication links require a beam-steerable antenna [1], [2]. The capability to compensate for the sensitivity of the antenna to the propagation environment conditions is another reason for implementing a beam-scanning antenna. Antenna designers lean towards electronic beam-scanning antenna due to its ease of implementation as compared to the frequency and mechanical beam-scanning antennas. As discussed in Chapter 2, electronic beamscanning can be achieved using PiN diodes, semiconductor switches, varactor diodes, or 
MEMS. In the proposed designs, GaAs varactor diode was implemented to function as a switch due to its appealing features such as small footprint, high switching speed, and low loss. Only a few commercially available GaAs varactor diodes operate in the mmwave frequency bands.

In this chapter, different designs for achieving electronic beam-scanning are introduced. The antenna structures, simulated, and measured results are investigated in the remaining sections of this chapter. A comparison among the proposed antennas and state-of-the-art reconfigurable antennas is also made.

\subsection{Reconfigurable Antennas Based on Periodic Cells with Negative Permittivity}

As stated in Section 5.1, different values for permittivity, permeability, and phase constant can be achieved by tuning circuit parameters of the CRLH LWAs. In other words, the permittivity and permeability of the CRLH antennas can be tuned artificially to control the radiation pattern. It should be noted that variations in permittivity and permeability can be achieved by tuning the equivalent shunt and series resonators of any structure. In other words, permittivity and permeability of even non-CRLH structures can be tuned. Furthermore, it should be noted that any combinations of series and shunt resonators do not necessarily represent CRLH structures. CRLH antennas are recognized from their dispersion responses [149]. If a negative phase constant (i.e., backward radiation) is achieved using fundamental Floquet mode, the proposed structure can be labeled as CRLH [149]. Overall, a reconfigurable antenna can be modeled with series and shunt resonators, but only called CRLH in specific conditions. The key point of implementing reconfigurable antennas is to achieve electronic beam-scanning, which can be obtained by tuning the shunt or series resonators. CRLH structures are mostly useful 
for suppressing the OSB issue (i.e., scanning the broadside), which is only resolved by satisfying the balance conditions [149].

In this section, a reconfigurable antenna based on periodic cells with negative permittivity is proposed. According to [121], periodic cells with negative permittivity can confine the field. To validate this idea, the via fence of an HMSIW LWA was replaced by an array of reconfigurable cells with negative permittivity, as presented in Fig. 5.1(a).

The proposed structure was realized on Rogers RT/Duroid 5880 substrate. The length, width, and thickness of the structure are $51 \mathrm{~mm}, 10 \mathrm{~mm}$, and $0.127 \mathrm{~mm}$, respectively. As stated in the previous chapters, the thinnest commercially available substrate was chosen for implementing the antenna. The width of the antenna was extracted from (3.1), while the radiating length obtained from (2.4). The periodic cells are demonstrated as purple squares. As presented in Fig. 5.1(b), each cell contains a cross-shape conducting strip with a dimension of $0.5 \mathrm{~mm} \times 0.5 \mathrm{~mm} \times 0.127 \mathrm{~mm}$. The cell size was chosen to achieve negative $\varepsilon_{r}$ by sweeping the bias voltage to confine the fields. The vias act as shunt inductors, while the varactor diode switch acts as a series capacitor. The bias circuitry was placed beneath the antenna. A $1 \mathrm{k} \Omega$ resistor and $1 \mathrm{pF}$ capacitor were used as RF and DC blocks, respectively. The electric field distribution is shown in Fig. 5.1(c) confirms the confinement of the field by periodic cells with negative permittivity. It was observed that field confinement was improved by making the permittivity more negative. Overall, replacing the via fence with negative $\varepsilon_{r}$ cells provides the adaptive control of the sidewall topology and radiation pattern. 


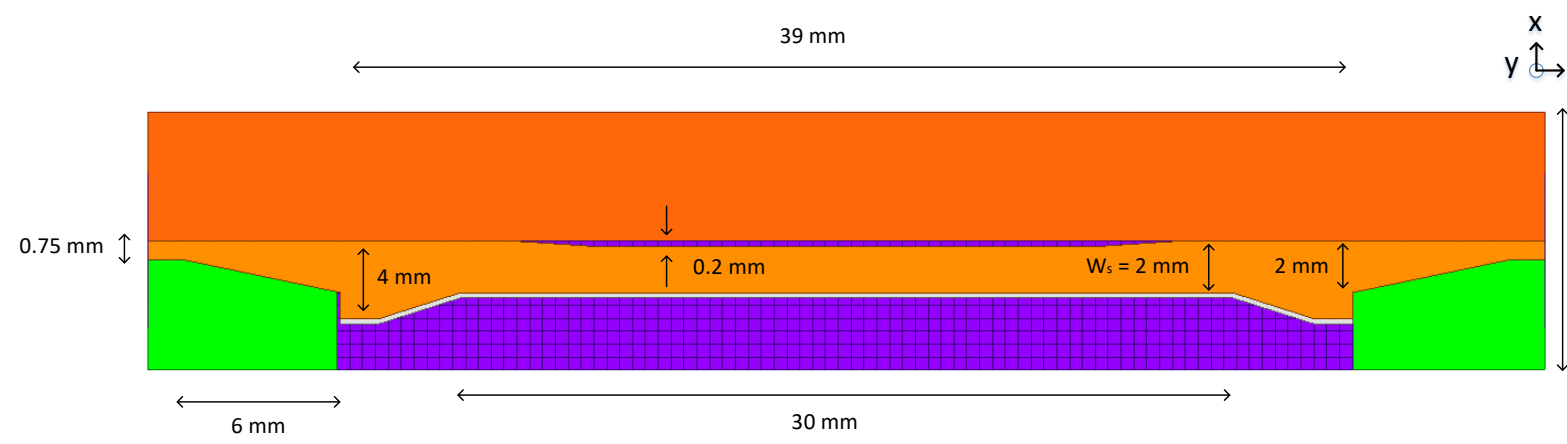

(a)

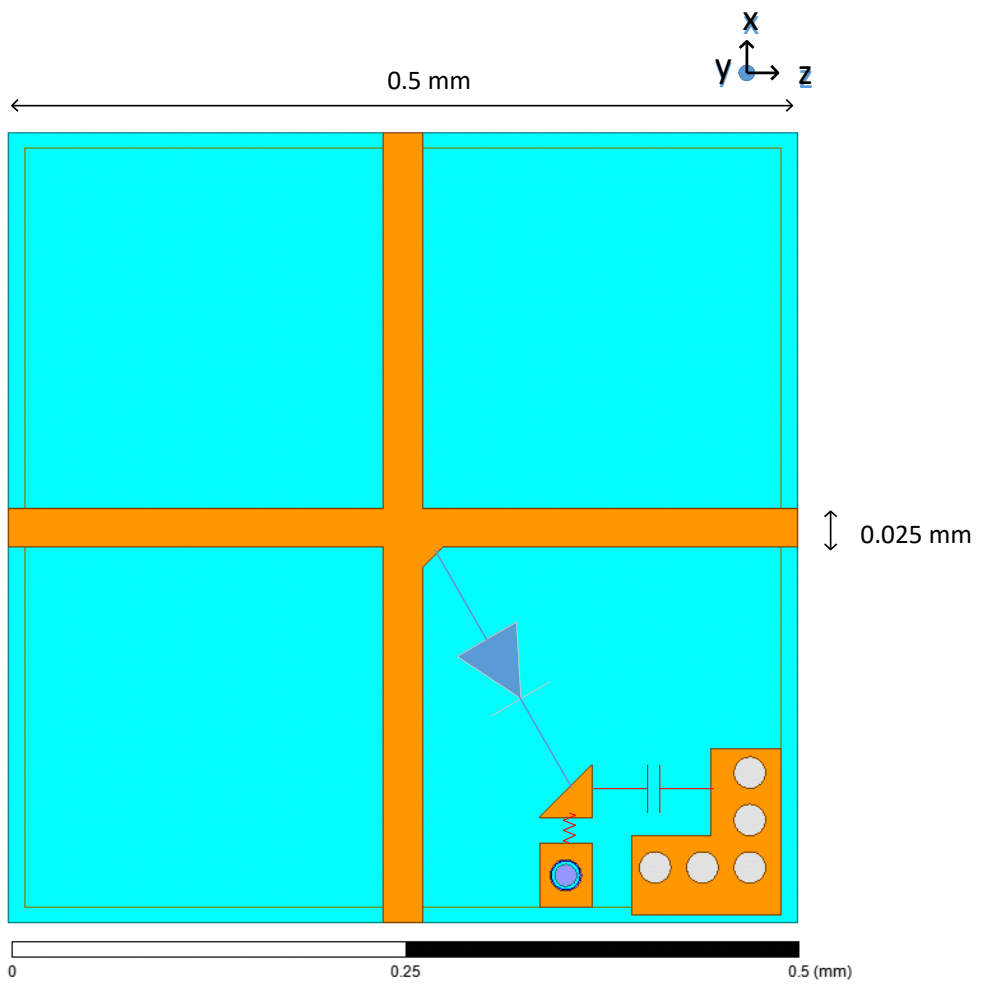

(b)

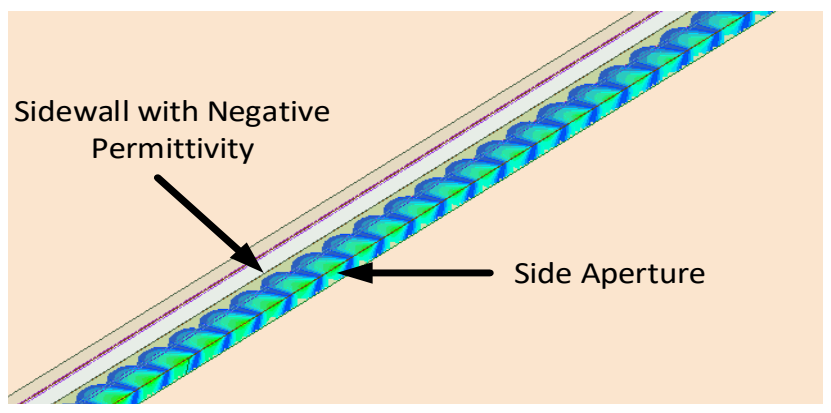

(c)

Figure 5.1. LWA with negative $\varepsilon_{r}$ cells. (a) top view of the proposed antenna, (b) schematic view of the reconfigurable cell, and (c) electric field distribution for cells with $\varepsilon_{r}=-100$. 
The permittivity and permeability of a reconfigurable cell versus frequency for reverse bias voltages of $0 \mathrm{~V}$ and $25 \mathrm{~V}$ are reported in Fig. 5.2. As observed in Fig. 5.2, sweeping the bias voltage leads to variations in permittivity and permeability. According to Fig. 5.2, negative $\varepsilon_{r}$ was achieved at $28.5 \mathrm{GHz}\left(\varepsilon_{r}=-38.79\right)$ for $\mathrm{V}_{\text {bias }}=-25 \mathrm{~V}$. However, the length of the proposed periodic cell size is about $\lambda_{0} / 20\left(\lambda_{\mathrm{g}} / 25\right)$. Unfortunately, realizing such small cells using state-of-the-art fabrication technologies (in either LTCC or PCB) is impossible. The smallest allowable cell size is about $\lambda_{0} / 5\left(\lambda_{\mathrm{g}} / 6\right)$. The minimum achievable $\varepsilon_{r}$ value for such size is about -2 , which is not enough for confining the field.

Preliminary simulations have been carried out using Ansys HFSS v.18. The Sparameter diagrams are presented in Fig. 5.3. The return loss is better than $15 \mathrm{~dB}$, and the insertion loss is between $4 \mathrm{~dB}$ and $7 \mathrm{~dB}$ over the $3 \mathrm{GHz}$ bandwidth ranging from $27 \mathrm{GHz}$ to $30 \mathrm{GHz}$ for $\mathrm{V}_{\text {bias }}=-25 \mathrm{~V}$. The radiation gain patterns of the antenna at $28.5 \mathrm{GHz}$ for different values of $\mathrm{W}_{2}$ (i.e., the distance of the sidewall from the side aperture) are reported in Fig. 5.4. Periodic cells with negative $\varepsilon_{r}$ resemble the sidewall. Changing the permittivity of the cells is similar to moving the sidewall and varying $\mathrm{W}_{2}$. This results in the variation of the cutoff frequency and propagation constant, which leads to electronic beam-scanning and variation in the realized gain, as demonstrated in Fig. 5.4. 

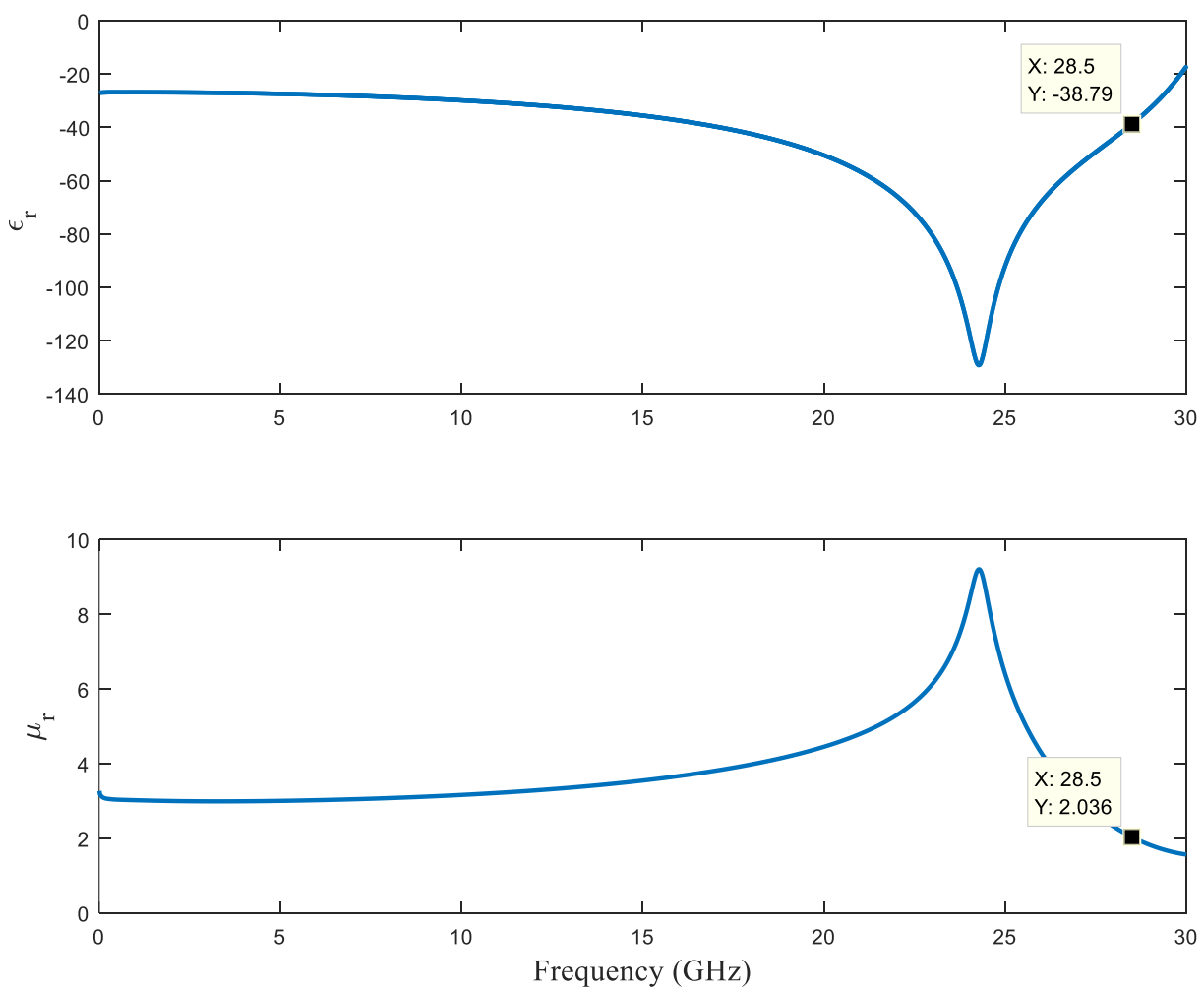

(a)
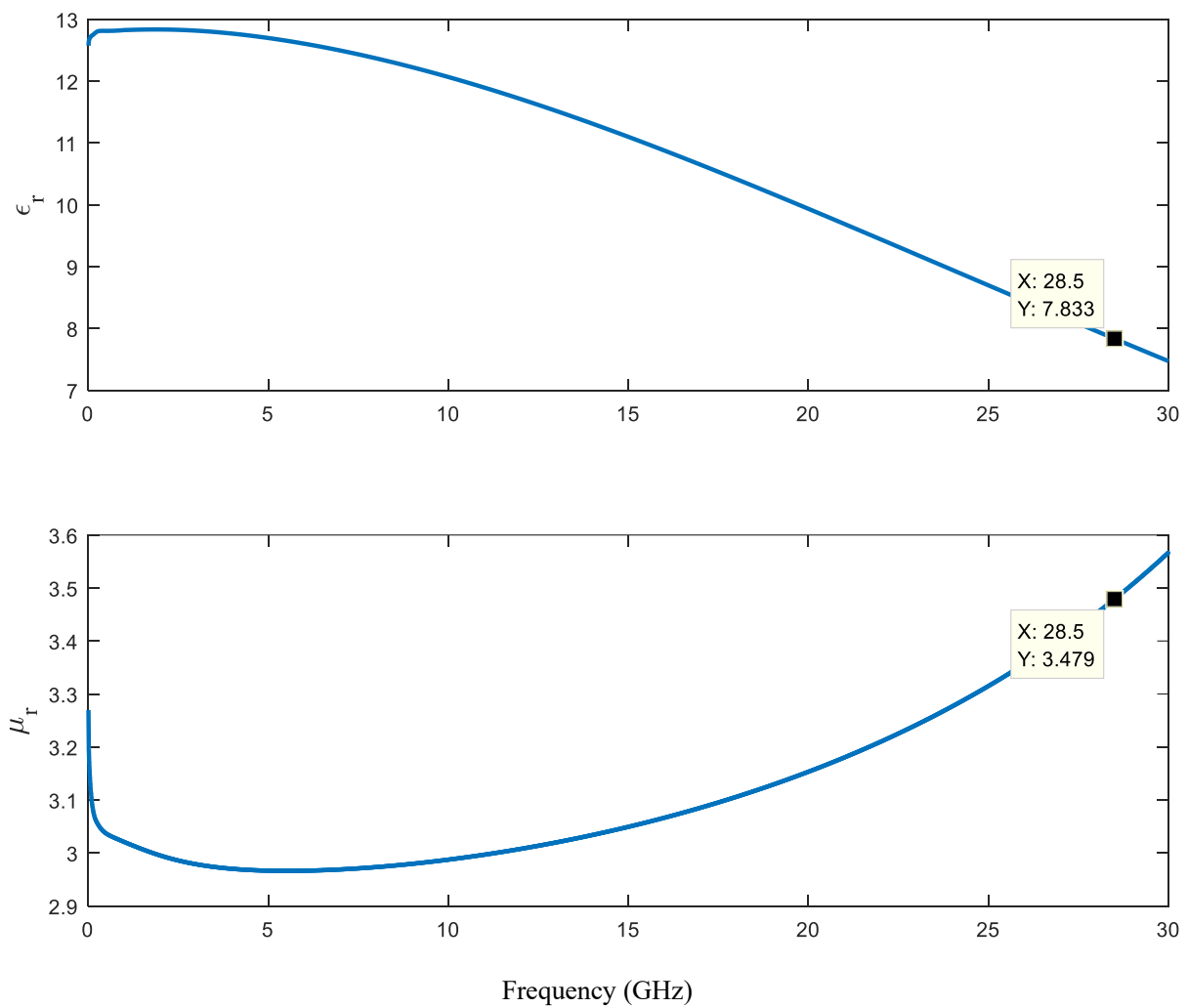

(b)

Figure 5.2. Permittivity and permeability of the reconfigurable cells versus frequency. (a) $\varepsilon_{r}$ and $\mu_{r}$ for $\mathrm{V}_{\text {bias }}=-25 \mathrm{~V}$, (b) $\varepsilon_{r}$ and $\mu_{r}$ for $\mathrm{V}_{\text {bias }}=0 \mathrm{~V}$. 


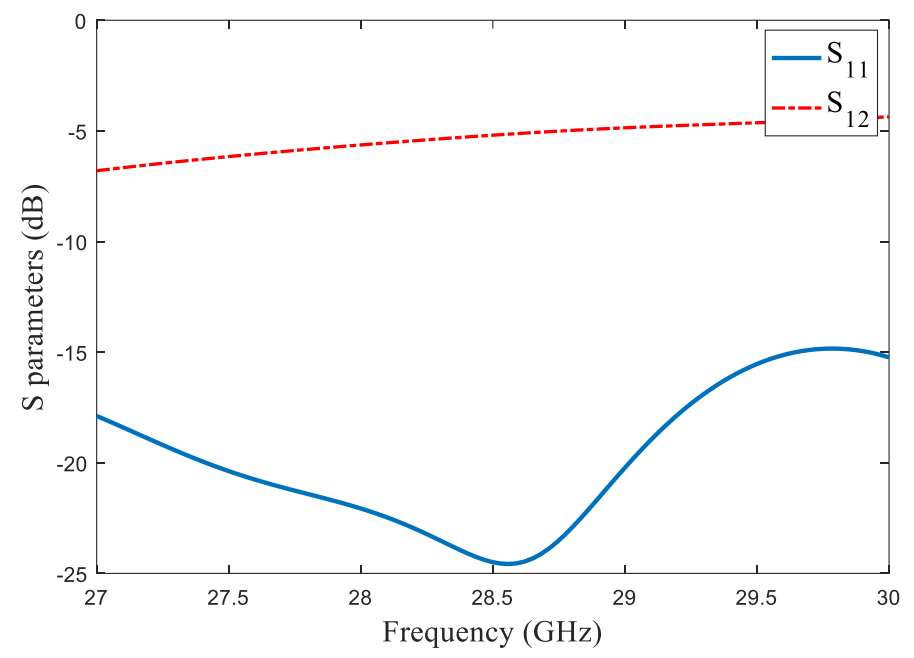

Figure 5.3. S-parameter diagrams of the proposed antenna with negative $\varepsilon_{r}$ cells at $\mathrm{V}_{\text {bias }}=\mathbf{2 5 \mathrm { v }}$.

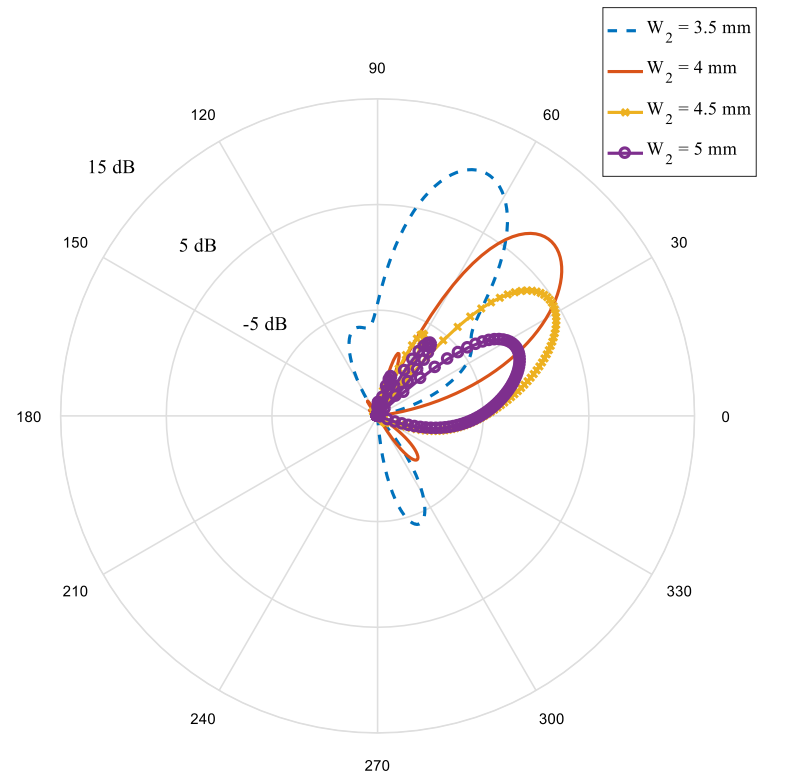

(a)

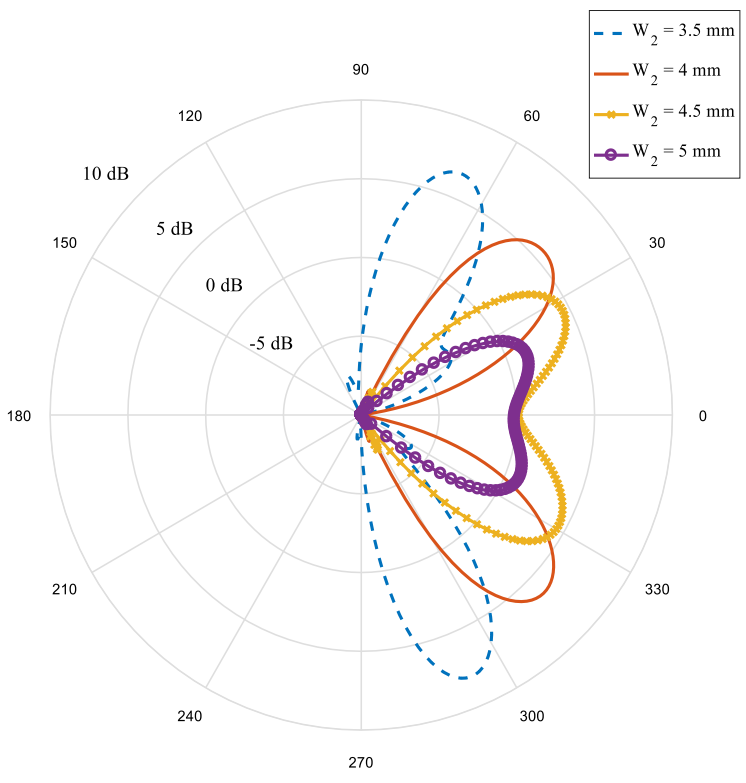

(b)

Figure 5.4. The radiation pattern of the proposed antenna for different values of $W_{2}$. (a) E-plane (YZ-plane), (b) XZ-plane.

As stated before, according to our investigations, realizing an HMSIW LWA based on the reported FPMS cells in [121] does not result in satisfactory radiation at high frequencies. This is due to the unwanted leakage from the cross-shape periodic cells. In other words, most of the incoming power leaks to space through the gaps between stubs. Reconfigurable circular- and rectangular-shape cells, with small spacing to reduce 
undesired leakage, will be implemented in the designs proposed in the next section. Since the designed cells are too small to be realized using the current fabrication technologies, the reconfigurable cells cannot confine the field. However, tuning a varactor diode of the proposed cell changes the impedance and resonance frequency, resulting in electronic beam-scanning.

\subsection{Waveguide-Based Reconfigurable Antennas}

Different types of reconfigurable antennas based on HMSIW and SIW are investigated in this section. The proposed designs are based on a multi-layer Rogers RT/Duroid 5880 substrate with $0.5 \mathrm{oz}$ copper cladding. The thickness of each layer was chosen as 0.127 $\mathrm{mm}$ to achieve a low-profile structure. The waveguide widths $(W)$ of the SIW-based antennas were obtained from (2.5) and (2.6) to achieve a single-mode propagation. Whereas the waveguide widths of the HMSIW-based structures were determined by the number, size, and spacing of the reconfigurable cells. The radiating lengths of the antennas were obtained from (2.4) to achieve the desired $\theta_{0}$ and $\Delta \theta$. As stated in Chapters 3 and 4 , the total length of the antenna depends on the length of the feed transition, which was optimized to achieve adequate return loss and realized gain.

In each of the proposed antennas, a tapered via fence in addition to the tapered microstrip transition was used to improve the impedance matching. Both ends of the antenna were also attached to RF connectors, while the output connector was terminated by a $50 \Omega$-matched load to absorb the remaining un-radiated power. All the simulations are carried out using Ansys Circuit and Ansys HFSS v.18-20.

In the SIW- and HMSIW-based reconfigurable antennas, MAVR000120-14110 [147] and MAVR011020-14110 [148] from MACOM company were chosen as the varactor 
diodes, respectively. To bias the varactor diode, the anode of the varactor diode was grounded through the side via fence, while the cathode was connected to the bias circuitry through an RF block. To achieve a low-profile design, the bias circuitry was placed underneath the ground plane of the antenna. Grounding the varactor diode through a DC block is necessary to complete the RF path and prevent significant reflection from it. Each reconfigurable cell can be modeled as shunt and series resonators. The via fence and varactor diode act as shunt inductor and series capacitor, respectively. The conductive plane and the dielectric layer sandwiched between top and ground planes act as series inductor and shunt capacitor, respectively. Sweeping the reverse bias voltage of the varactor diode results in tuning the series capacitance. This changes the impedance of the cell and propagation constant that results in electronic beam-scanning. The HPBW is also altered by switching since the HPBW of an LWA depends on $\beta$ and $\theta_{0}$ [28]. Hence, directivity and gain are varied by tuning the bias voltage.

Switching also affects the return loss and insertion loss due to the variations of the capacitance and impedance. To minimize the effects of switching on the impedance matching, reconfigurable cells were only realized in the radiation section of the antenna. In the preliminary designs, the switches were located over the slots, blocking parts of the slots due to their footprints. Switching has a similar effect as changing the size of the slots. In the last few designs and the fabricated antennas, switches were placed on the backside of the antennas to resolve the footprint blockage effect. It is also possible to place the switches outside the radiation section and the via fence. However, since the fields are confined by the via fences, each switch must connect to the radiating section 
through the wire bonding, which is lossy and inductive. Doing so also requires increasing the width of the structure that is not desirable.

The switches can be biased uniformly or non-uniformly. Uniform biasing means the switches are biased at the same voltage level, while non-uniform biasing is achieved by applying different voltages to the switches. Switches can be controlled independently by non-uniform biasing, provides more control on the radiation pattern. However, this is achieved at the expense of increased complexity and cost of bias circuitry.

\subsubsection{Reconfigurable HMSIW LWA with Patches}

The first proposed antenna is an HMSIW LWA with an array of patches implemented on a three-layer Rogers RT/Duroid 5880. The antenna scans space electronically by introducing sets of switches over the patches on the top conductor plane. Sweeping the reverse bias voltages disturbs the field distribution that leads to beam-steering. The proposed antenna is presented in Fig. 5.5. The total length, width, and thickness of the antenna are $68 \mathrm{~mm}, 25 \mathrm{~mm}$, and $0.3 \mathrm{~mm}$, respectively. In the proposed design, only two states are considered for each varactor diode. The "on" state refers to the upper limit of capacitance range, $0.5 \mathrm{pF}$, and the "off" state corresponds to the lower limit, $0.05 \mathrm{pF}$. The reconfigurable section consists of fifteen transverse lines with two patches per line. Hence, forty-five switches are required. All the switches in a row along the longitudinal axis are biased similarly to reduce the complexity and effect of switching on the impedance matching. Switching changes the configuration of the patch arrays. This leads to the variations in phase constant resulting in beam-scanning.

In this antenna, radiation occurs from the side aperture and the patches. By turning "on" the switches in each row, the current will pass to the next one. This scenario is the 
same as changing the dimension of the microstrip patch. From the LWA perspective, connecting/disconnecting patches is similar to changing the dimensions of the slots. From the array antenna standpoint, switching has the same effect as changing the number of the array elements [100]. The S-parameters for different switching states at $28.5 \mathrm{GHz}$ are presented in Table 5.1. The return loss is better than $10 \mathrm{~dB}$ in all states, while the insertion loss ranges from 4 to $7.3 \mathrm{~dB}$ representing the low residual power.

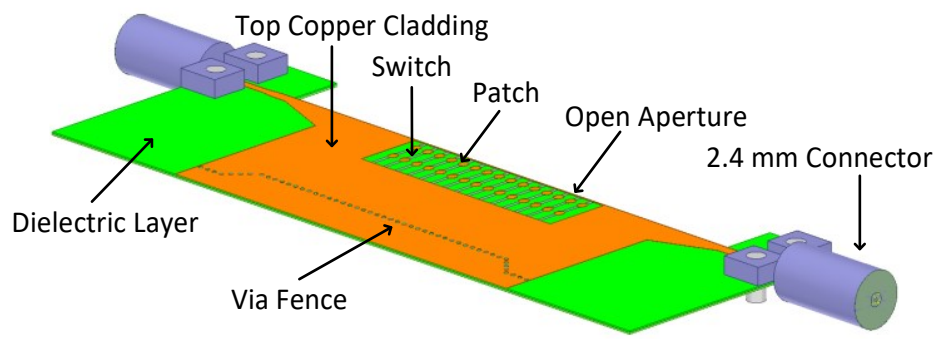

(a)

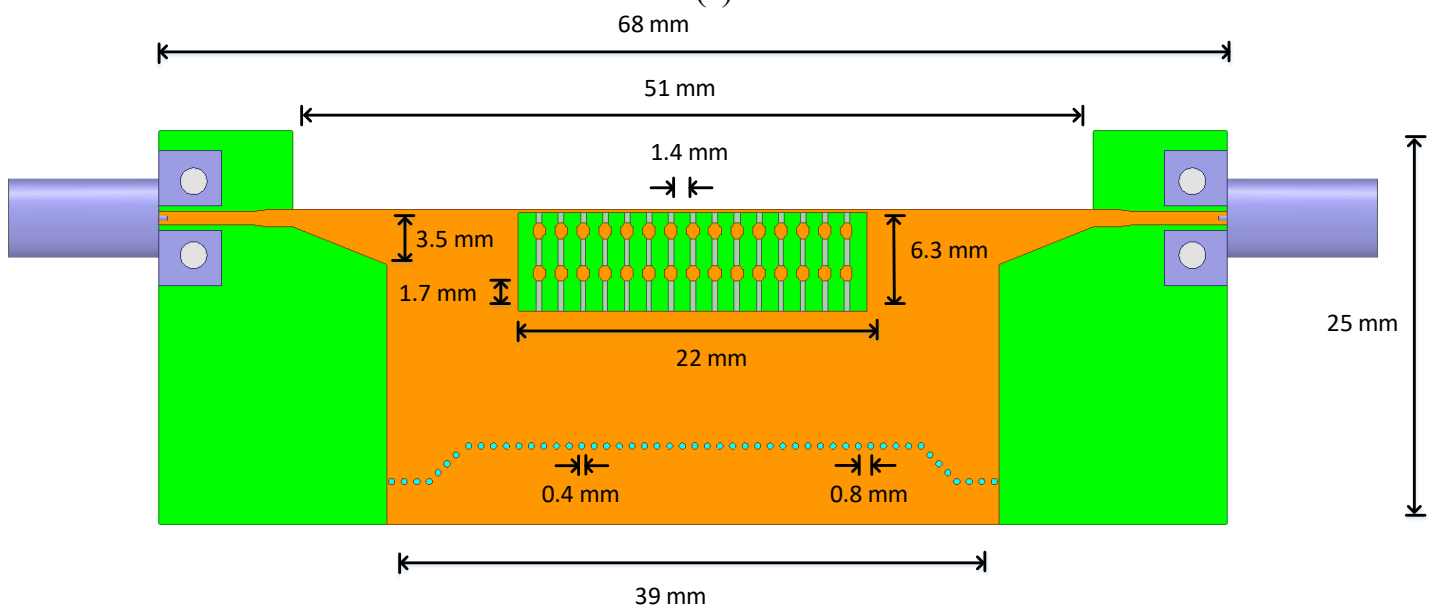

(b)

Figure 5.5. Schematic view of the reconfigurable HMSIW LWA with patches. (a) 3D view, (b) top view [100].

Table 5.1. S-parameters of the HMSIW LWA with patches for different switching states [100].

\begin{tabular}{|c|c|c|}
\hline Switches States & $\mathbf{S}_{\mathbf{1 1}}$ & $\mathbf{S}_{\mathbf{2 1}}$ \\
\hline off-off-off & $-10.2 \mathrm{~dB}$ & $-5.4 \mathrm{~dB}$ \\
\hline on-off-off & $-10.1 \mathrm{~dB}$ & $-7.3 \mathrm{~dB}$ \\
\hline off-off-on & $-10 \mathrm{~dB}$ & $-4 \mathrm{~dB}$ \\
\hline on-on-on & $-20.9 \mathrm{~dB}$ & $-5 \mathrm{~dB}$ \\
\hline
\end{tabular}


The radiation patterns of the antenna for different switching states are shown in Fig. 5.6, confirming the electronic beam-scanning of about $50^{\circ}$. The proposed reconfigurable antenna's beam scans from $75^{\circ}$ to $125^{\circ}$ with relatively low gain ranging from $0.3 \mathrm{dBi}$ to 4.6 dBi. The small thickness of the antenna results in a high conductive loss that degrades the gain. In addition, the reported gain is the realized gain depends on the S-parameters. Hence, non-optimum return loss can result in such low gain.

\subsubsection{Reconfigurable HMSIW LWA with Circular Slots}

In this section, implementing reconfigurable circular slots with switches on HMSIW LWAs leads to electronic beam-scanning. Each reconfigurable cell contains a varactor diode, DC block, and RF block arranged over a circular slot. As presented in Fig. 5.7, several topologies for the cells were considered. The circular shape was chosen as the best candidate due to its smooth edge, resulting in a mild disturbance in the surface current.

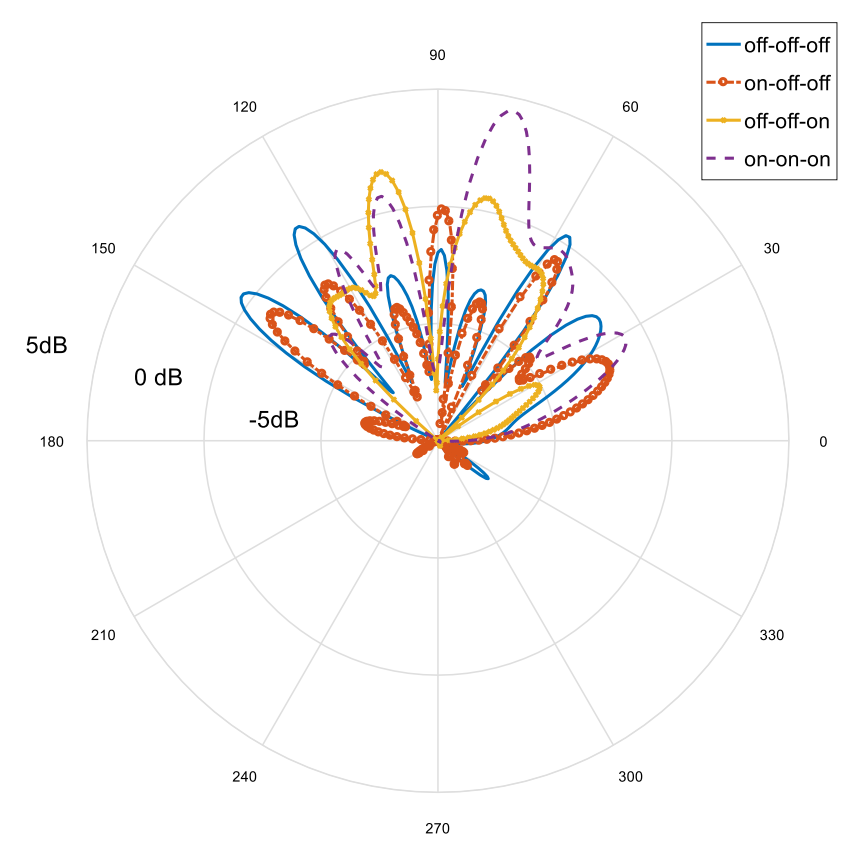

Figure 5.6. Radiation patterns of the reconfigurable HMSIW LWA with patches for different switching states and E-plane $\left(\varphi=9^{\circ}\right)[100]$. 


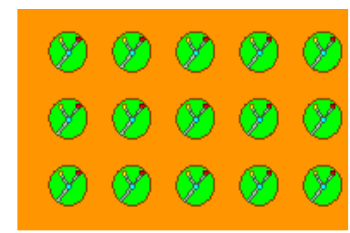

(a)

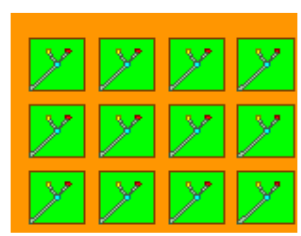

(b)

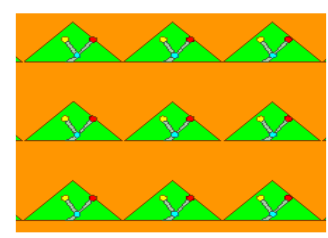

(c)

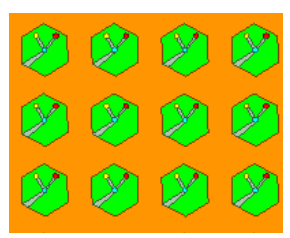

(d)

Figure 5.7. Different topologies for the reconfigurable cells. (a) circular shape, (b) square shape, (c) triangular shape, and (d) hexangular shape.

The proposed antennas were implemented on a three-layer Rogers RT/Duroid 5880 with the total thickness of $0.3 \mathrm{~mm}$ to achieve a low-profile structure. In other words, each of the proposed antennas contains two Rogers RT/Duroid 5880 board with the thickness of $0.127 \mathrm{~mm}$. The first proposed reconfigurable HMSIW antenna is presented in Fig. 5.8 and contains thirty-five cells. The length, width, and thickness of the proposed antenna are $69 \mathrm{~mm}, 45 \mathrm{~mm}$, and $0.3 \mathrm{~mm}$, respectively. The length of the antenna was adjusted to achieve a relatively high directivity while keeping the antenna size reasonably small. Since the antenna is realized on an HMSIW, radiation occurs from both the circular slots and the side aperture. Only two switching states are considered; the "on" and "off" states correspond to the varactor capacitance values of $0.5 \mathrm{pF}$ and $0.05 \mathrm{pF}$, respectively.

The second proposed HMSIW LWA is presented in Fig. 5.9. The proposed antenna contains thirty-five circular slots with a diameter of $3.4 \mathrm{~mm}$ in a V-shape topology. Switches in a V-shape row are biased uniformly. The length, width, and thickness of the antenna are $67 \mathrm{~mm}, 45 \mathrm{~mm}$, and $0.3 \mathrm{~mm}$, respectively. 


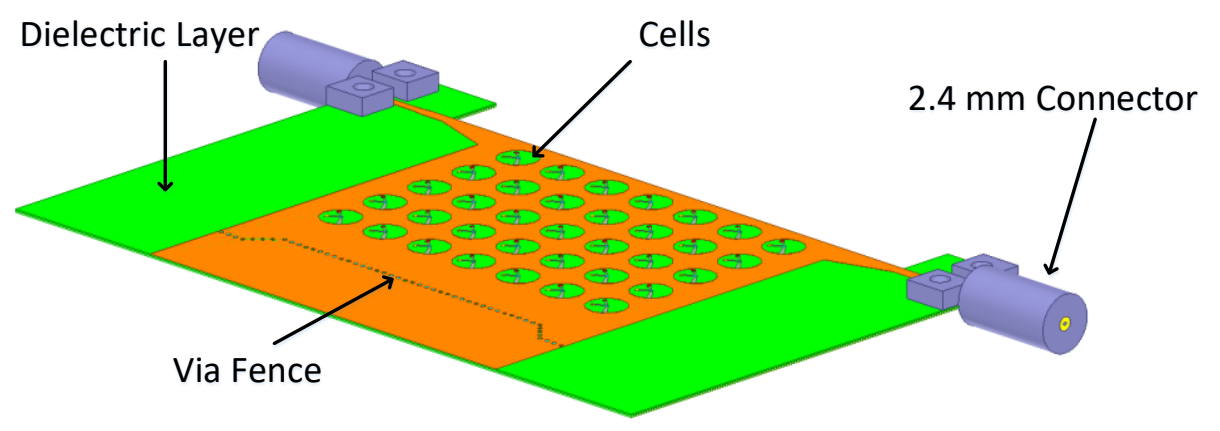

(a)

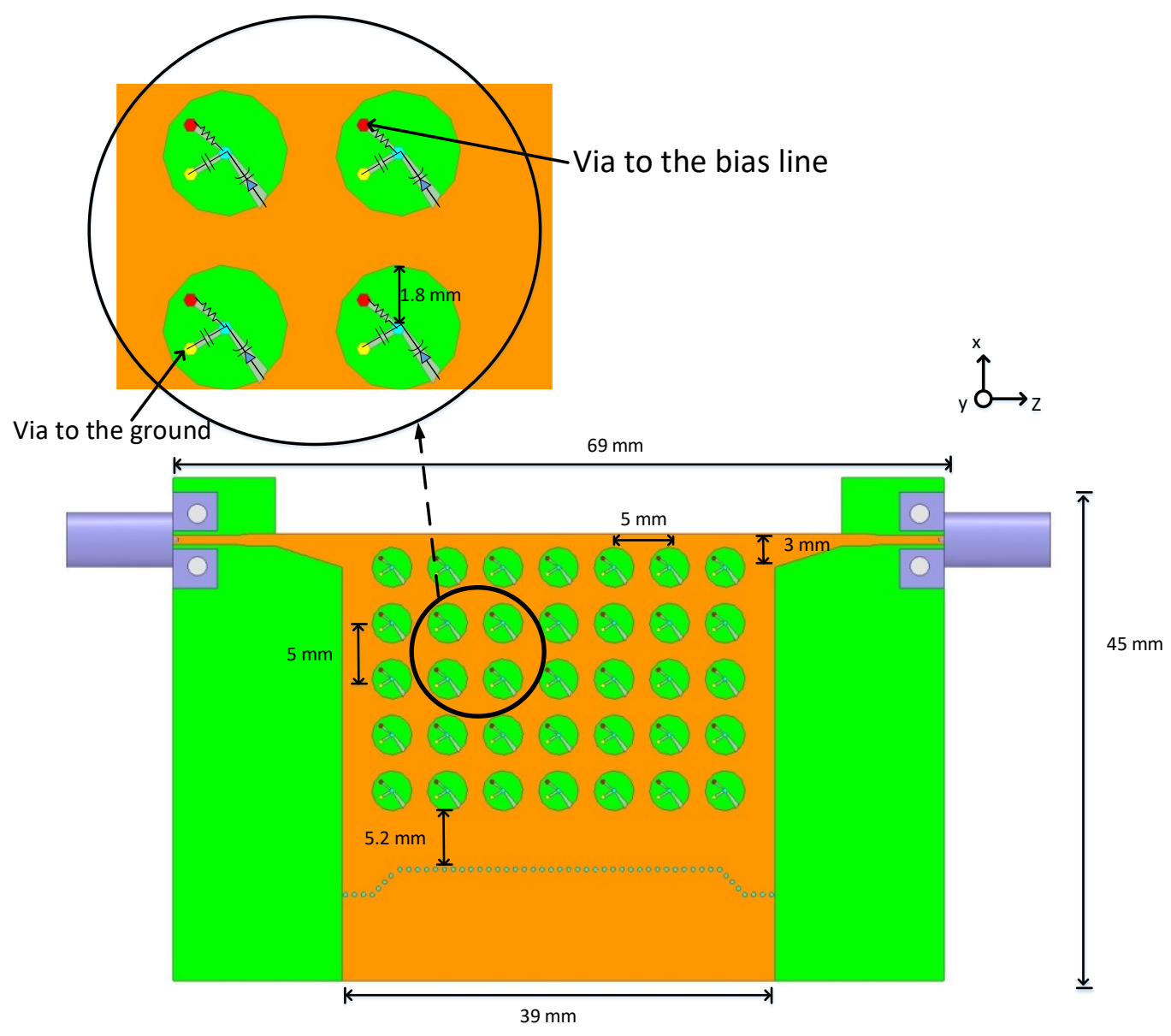

(b)

Figure 5.8. Schematic view of the reconfigurable HMSIW LWA with circular slots [101]. (a) 3D view, (b) top view. 


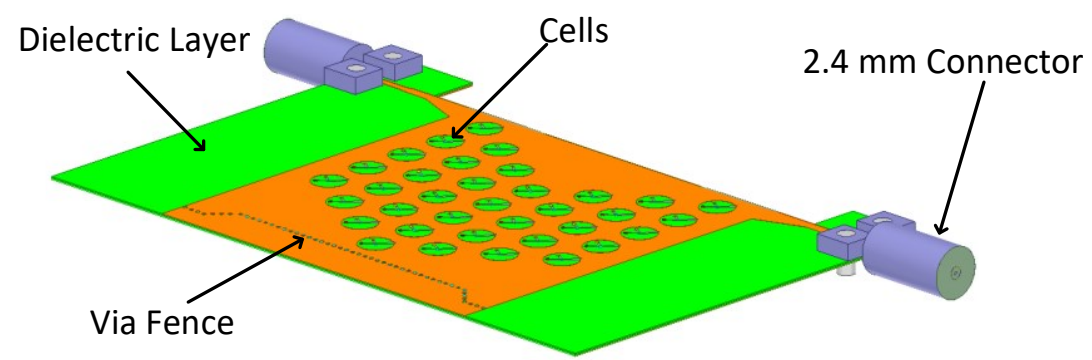

(a)

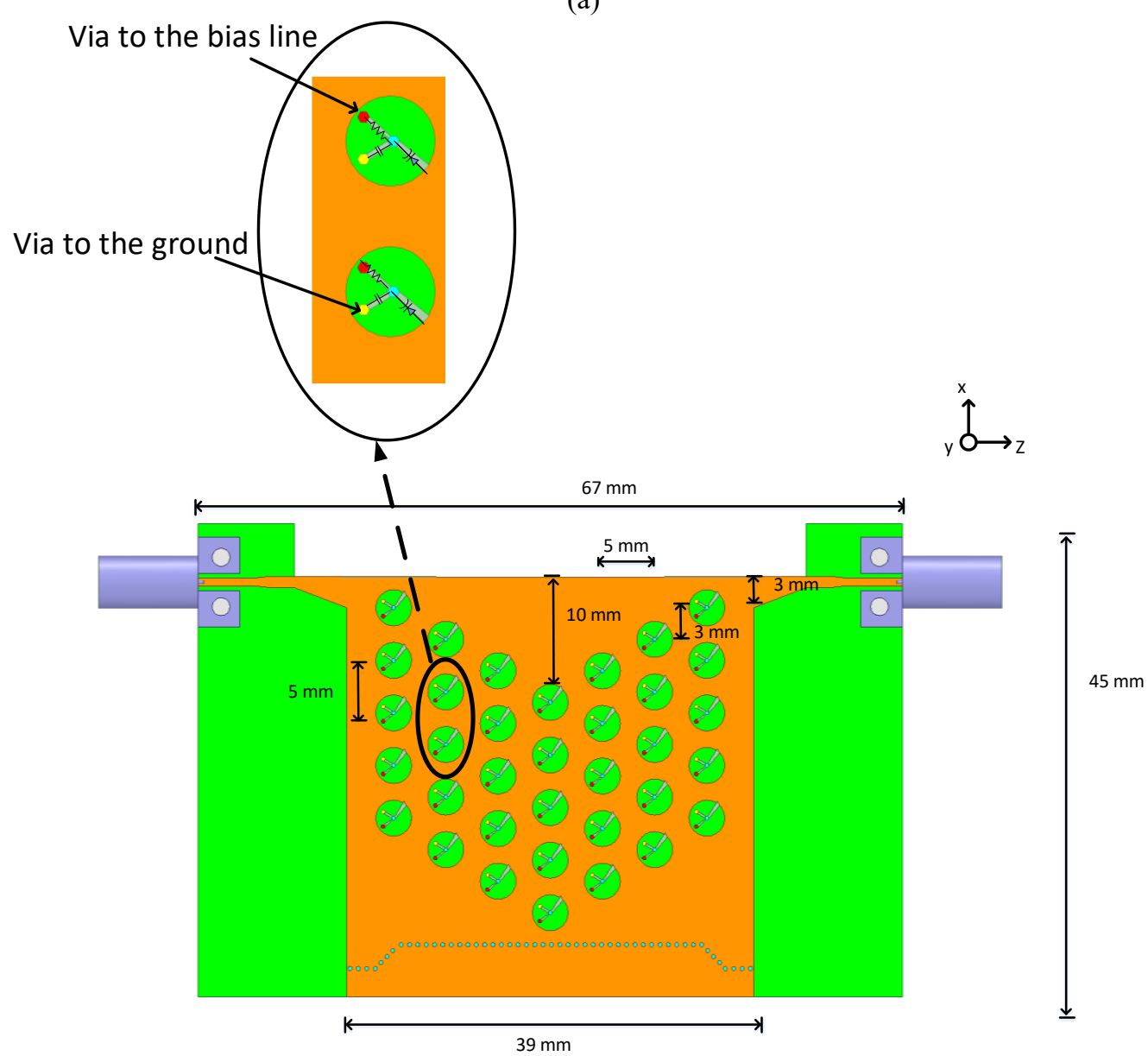

(b)

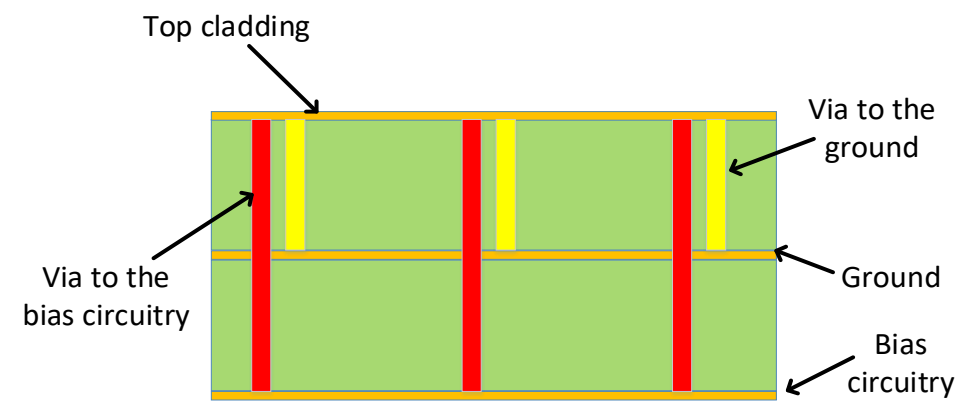

(c)

Figure 5.9. Schematic view of the reconfigurable HMSIW LWA with circular slots in V-shape topology [102]. (a) 3D view, (b) top view, and (c) side view. 
Switching each cell individually provides more control over the radiation pattern at the expense of complex bias circuitry. Hence, to avoid complexity of the bias circuitry, all the switches were biased uniformly. Multiple switching topologies can be implemented to achieve electronic beam-scanning. Here, longitudinal and transversal switching topologies for the first HMSIW LWA are investigated. In the longitudinal configuration, all switches in a row along the z-axis are biased similarly. In the transversal topology, switches in a column along the x-axis are connected to the same bias voltage. Longitudinal and transversal switching dominantly disturb transverse and longitudinal surface currents, respectively. This is because longitudinal and transversal switching can be approximately modelled as shifting the longitudinal and transverse slots, respectively. Longitudinal switching is similar to changing the width of the HMSIW artificially. First, the results of longitudinal switching are presented. Next, the results of the transversal configuration are investigated. Finally, the results of the V-shape topology with uniform switching are discussed.

The S-parameters of the antenna configured with longitudinal switching are presented in Fig. 5.10. It exhibits a return loss better than $10 \mathrm{~dB}$ and confirming proper impedance matching from $28.1 \mathrm{GHz}$ to $28.7 \mathrm{GHz}$ in all states. The insertion loss ranges from $3 \mathrm{~dB}$ to $11 \mathrm{~dB}$ over the frequency band of 28.1-28.7 GHz. The radiation patterns for different longitudinal switching states are shown in Fig. 5.11, indicating the electronic beamsteering. The beam-scanning range of the longitudinal switching HMSIW LWA is about $13^{\circ}$, while the peak realized gain ranges from $2.8 \mathrm{dBi}$ to $8.6 \mathrm{dBi}$. To investigate the validity of the proposed design, the results of only four states are reported; (1) all 
switches off, (2) all switches on, (3) nearest row to the aperture (first row) off, and (4) closest row to the via fence (last row) off.

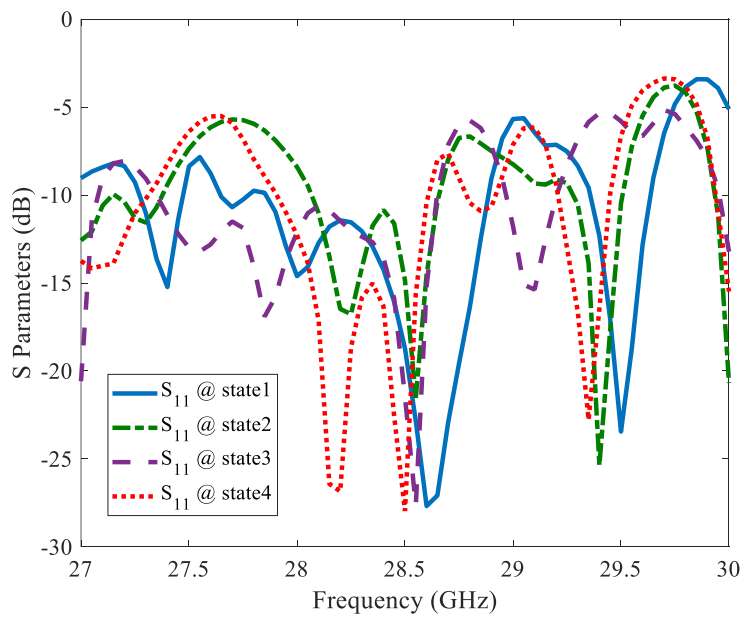

(a)

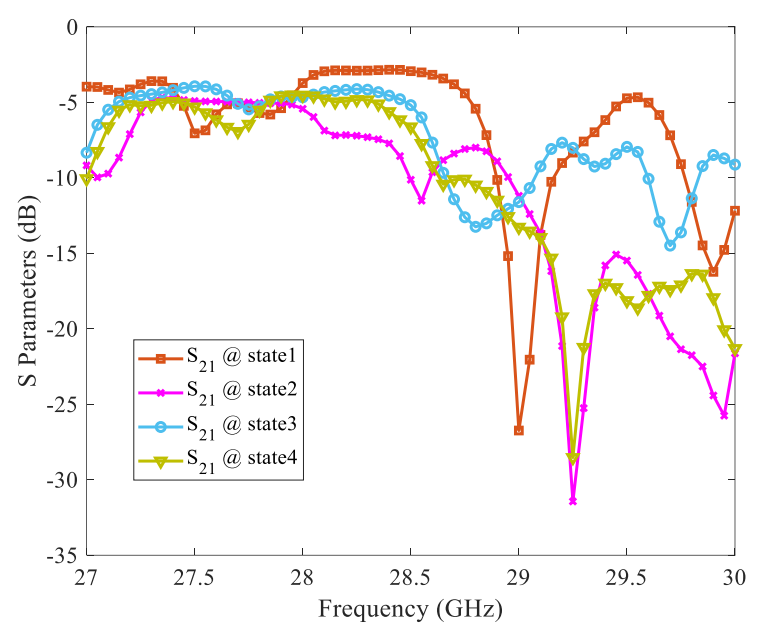

(b)

Figure 5.10. S-parameters of the proposed reconfigurable HMSIW antenna for different longitudinal switching states. (a) $S_{11}$, (b) $S_{21}$.

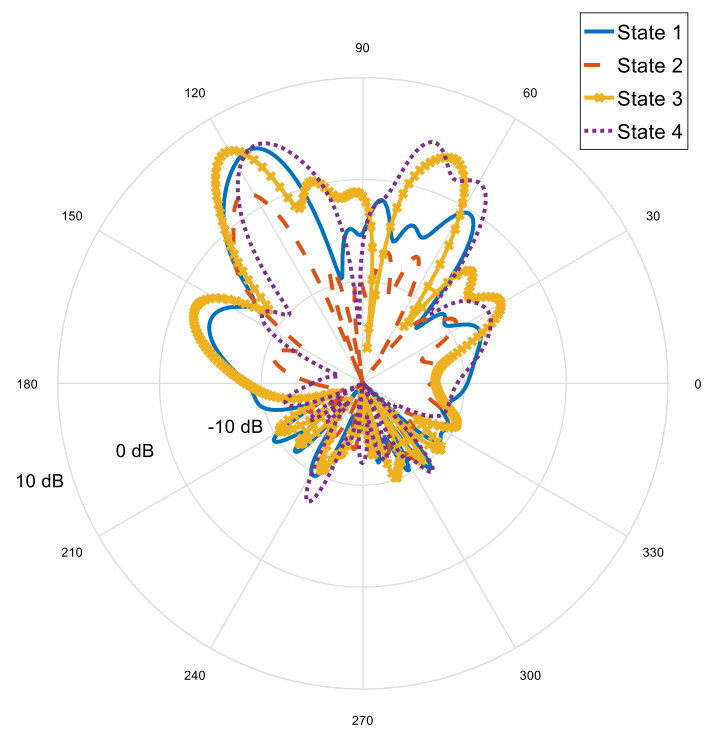

Figure 5.11. Radiation patterns of reconfigurable HMSIW LWA with circular slot and different longitudinal switching states at $28.5 \mathrm{GHz}$ and E-plane; (1) all on, (2) all off, (3) the first row (nearby the aperture) off, and (4) the last row (nearby the via fence) off. 
Next, the results of transversal switching are presented. The S-parameters for different switching states are presented in Fig. 5.12, exhibiting return loss better than $10 \mathrm{~dB}$ in all states from $28.3 \mathrm{GHz}$ to $28.6 \mathrm{GHz}$. The corresponding insertion loss is altering from $3 \mathrm{~dB}$ to $9 \mathrm{~dB}$ at $28.5 \mathrm{GHz}$ for different switching states, demonstrating the variation in the power reaches the output port. The radiation patterns for different switching states are presented in Fig. 5.13 that shows scanning of the main-beam through space. The proposed HMSIW LWA with transverse switching achieves the beam-scanning range of about $15^{\circ}$ with the peak realized gain ranges from $2.7 \mathrm{dBi}$ to $8.8 \mathrm{dBi}$.

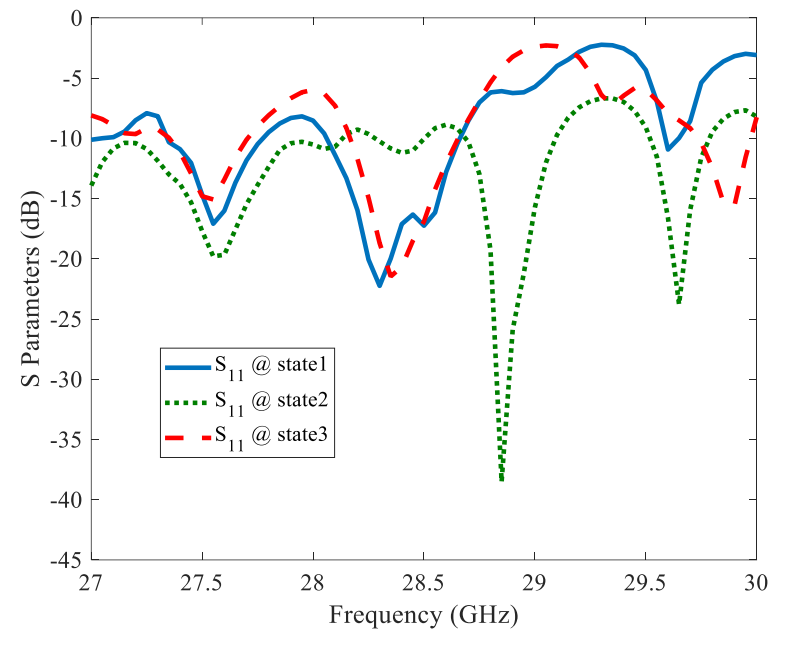

(a)

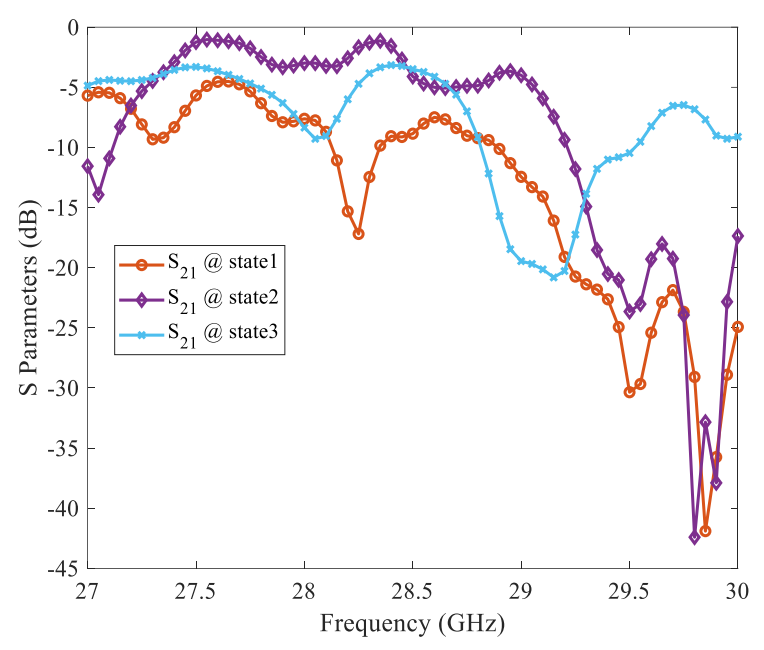

(b)

Figure 5.12. S-parameters of the proposed HMSIW LWA for different transversal switching states [101]. (1) the first column (closest to the input) off, (2) the last column (closest to the output) off, and (3) all off. (a) $S_{11}$, (b) $S_{21}$. 


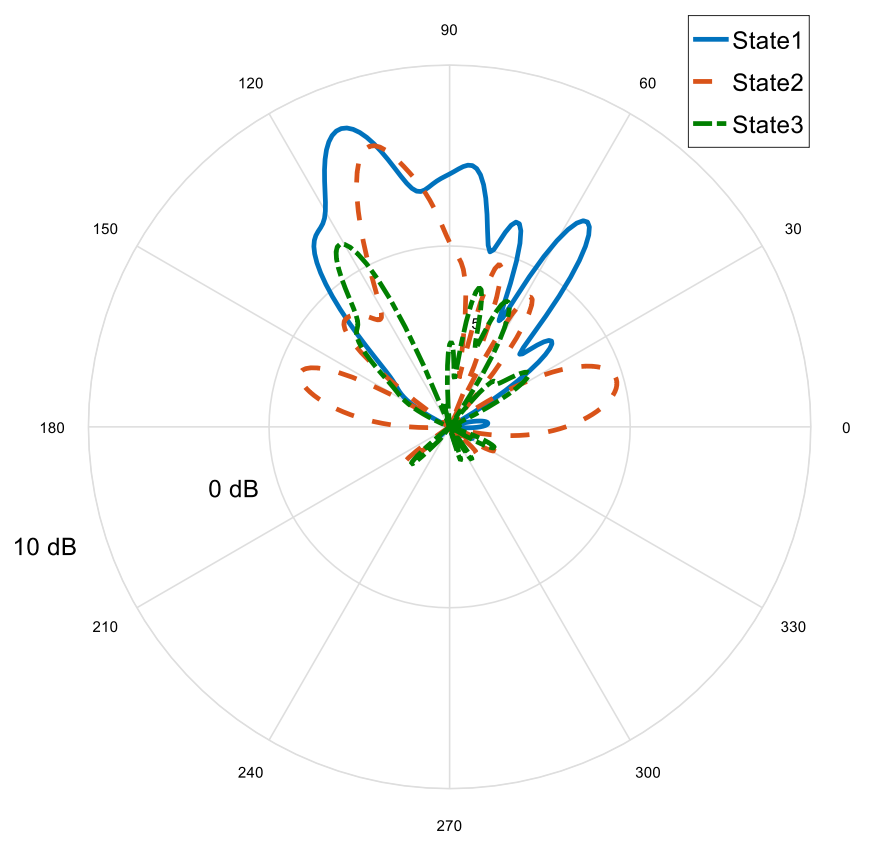

Figure 5.13. Radiation patterns of the reconfigurable HMSIW LWA with circular slot and transversal switching at $28.5 \mathrm{GHz}$ [101].

Finally, the results of the V-shape configuration with longitudinal switching topology were investigated. The S-parameters for different switching states are presented in Fig. 5.14, exhibiting return loss better than $10 \mathrm{~dB}$ over the frequency range from $28.25 \mathrm{GHz}$ to 28.75 GHz. The insertion loss varies from $7 \mathrm{~dB}$ to $18 \mathrm{~dB}$ over the corresponding frequency band for different switching states. Furthermore, the insertion loss for different switching states at $28.5 \mathrm{GHz}$ ranges from $14 \mathrm{~dB}$ to $18 \mathrm{~dB}$. The radiation patterns for four different switching states are presented in Fig. 5.15, indicating the electronic beamscanning with medium gain. The investigated switching states are as follows; (1) all switches on, (2) all switches off, (3) row beside the aperture (first row) off, and (4) two nearest rows to the via fence (two last rows) off. The proposed antenna's beam scans $20^{\circ}$ while the peak realized gain ranges from $7.1 \mathrm{dBi}$ to $8.2 \mathrm{dBi}$. 


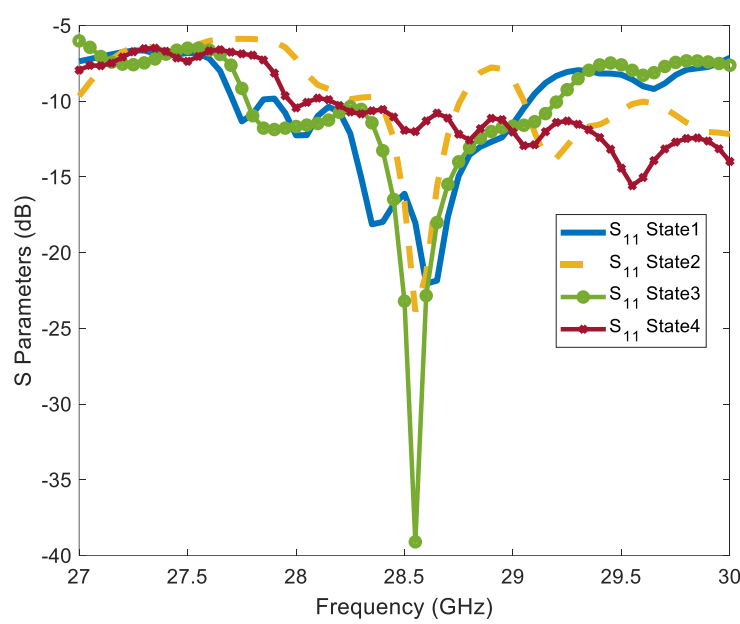

(a)

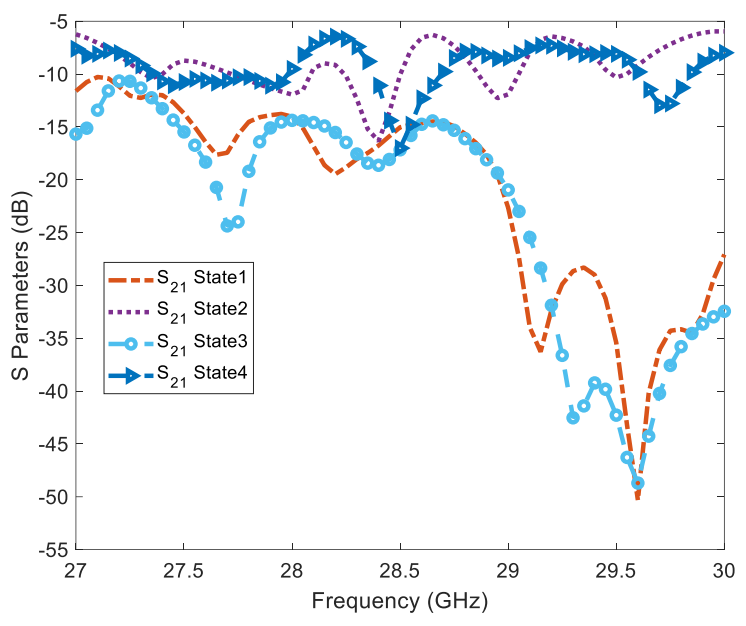

(b)

Figure 5.14. S-parameters of the proposed reconfigurable HMSIW LWA with V-shape configuration [102]. (a) $S_{11}$, (b) $S_{21}$.

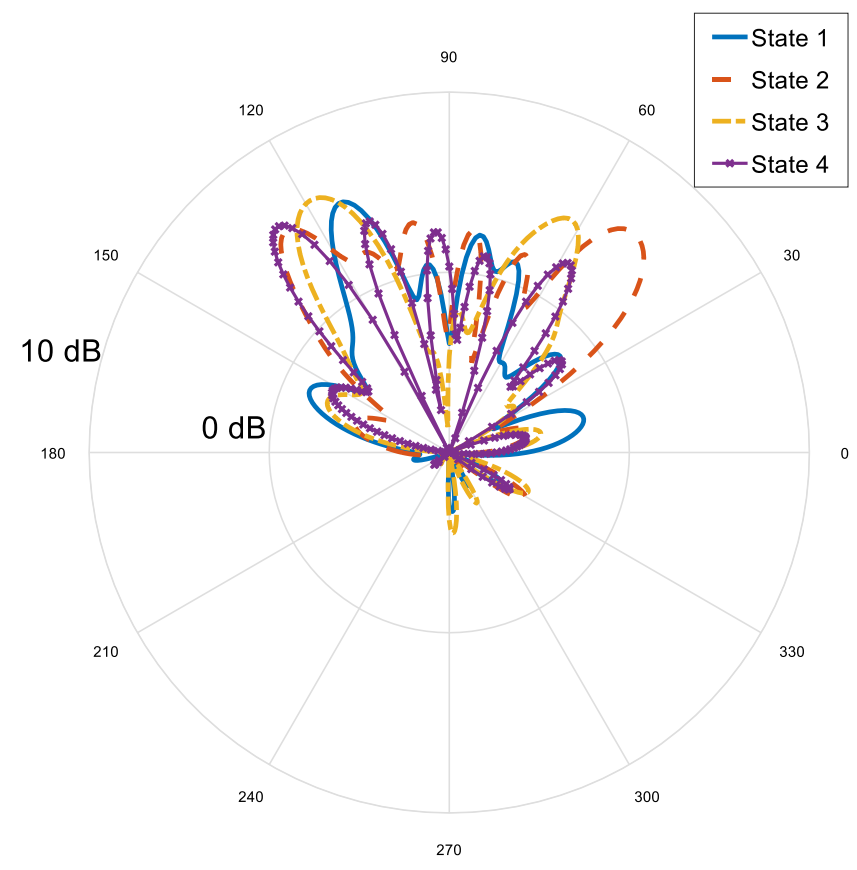

Figure 5.15. Radiation patterns of the reconfigurable HMSIW LWA with V-shape topology for different switching states at 28.5 GHz and E-plane (YZ plane); (1) all switches on, (2) all switches off, (3) the row close by the aperture (first row) off, and (4) the two nearest rows to the via fence (two last rows) off [102]. 


\subsubsection{HMSIW LWA with Reconfigurable Cells in V-Shape Configuration on the Backside}

In this section, a reconfigurable HMSIW LWA is demonstrated. The beam-steering is achieved using embedded varactor diodes in a V-shape configuration located on the backside of the antenna. Sweeping the reverse bias voltage causes variations of the phase constant, which leads to the electronic beam-scanning. The main features of the proposed reconfigurable antenna can be listed as the following: small gain variation, operation in the mm-wave frequency band, compactness, and backward beam-scanning with adequate scanning range using a single varactor diode per cell. These features make the proposed antenna a suitable candidate for 5G blind-spot monitoring and V2X communications.

The proposed antenna is a reconfigurable LWA based on HMSIW, as shown in Fig. 5.16. S-parameters of a single cell of the structure and $\beta$ are related by

$$
\cosh ((\alpha+j \beta) L)=\frac{S_{12} S_{21}+\left(1+S_{11}\right)\left(1-S_{22}\right)}{2 S_{21}}
$$

where $\alpha$ and $L$ are the attenuation constant and length of the cell, respectively [137]. According to (2.3) and (5.1), sweeping the varactor diode's reverse bias voltage leads to variations in capacitance of each reconfigurable cell, thereby changing the S-parameters, $\beta$, and $\theta_{0}$. In other words, switching leads to the electronic beam-scanning at a fixed frequency. It should be noted that (5.1) only provides a general guideline on the beamscanning mechanism since the proposed antenna is a quasi-periodic structure due to the $\mathrm{V}$-shape configuration of the reconfigurable cells.

As for other antennas previously investigated in this dissertation, the length of the antenna depends on the sizes of the radiation section and the feed. The feed length is adjusted to optimize the return loss of the antenna, while the radiation length is calculated 
by (2.4). The $\theta_{0}, \Delta \theta$, and center frequency were chosen as $130^{\circ}, 20^{\circ}$, and $28.5 \mathrm{GHz}$, respectively. This led to $L_{r}=39 \mathrm{~mm}$ based on (2.4). It should be noted that forward and backward endfires are at $\theta=0^{\circ}$ and $180^{\circ}$, respectively. In this design, $\theta_{0}$ was chosen as $130^{\circ}$ to achieve backward radiation, which is useful for monitoring blind spots in an autonomous vehicle.

The proposed reconfigurable antenna was implemented on a four-layer Rogers RT/Duroid 5880 substrate with relative permittivity of 2.2, small loss tangent of 0.0009 , and $0.5 \mathrm{oz}$ copper cladding. The total length, width, and thickness of the antenna are 67 $\mathrm{mm}, 48 \mathrm{~mm}$, and $0.32 \mathrm{~mm}$, respectively. The designed HMSIW LWA is composed of reconfigurable cells in a V-shape configuration, as shown in Fig. 5.16. Each reconfigurable cell includes a circular slot at the top plane and a GaAs varactor diode, DC block, and RF block on the backside of the structure. The proposed structure contains four copper claddings, where the second and the third copper claddings were merged to create the ground plane, as shown in Fig. 5.16(b). Some of the important geometrical parameters are listed in Table 5.2. A tapered via fence in addition to the tapered microstrip transition was implemented to minimize the return loss. Each end of the structure was terminated by a k-connector, while one of them was terminated by a $50 \Omega$ matched load to absorb the residual power since at least $10 \%$ of the input power should remain in the guided structure [28], [29]. 


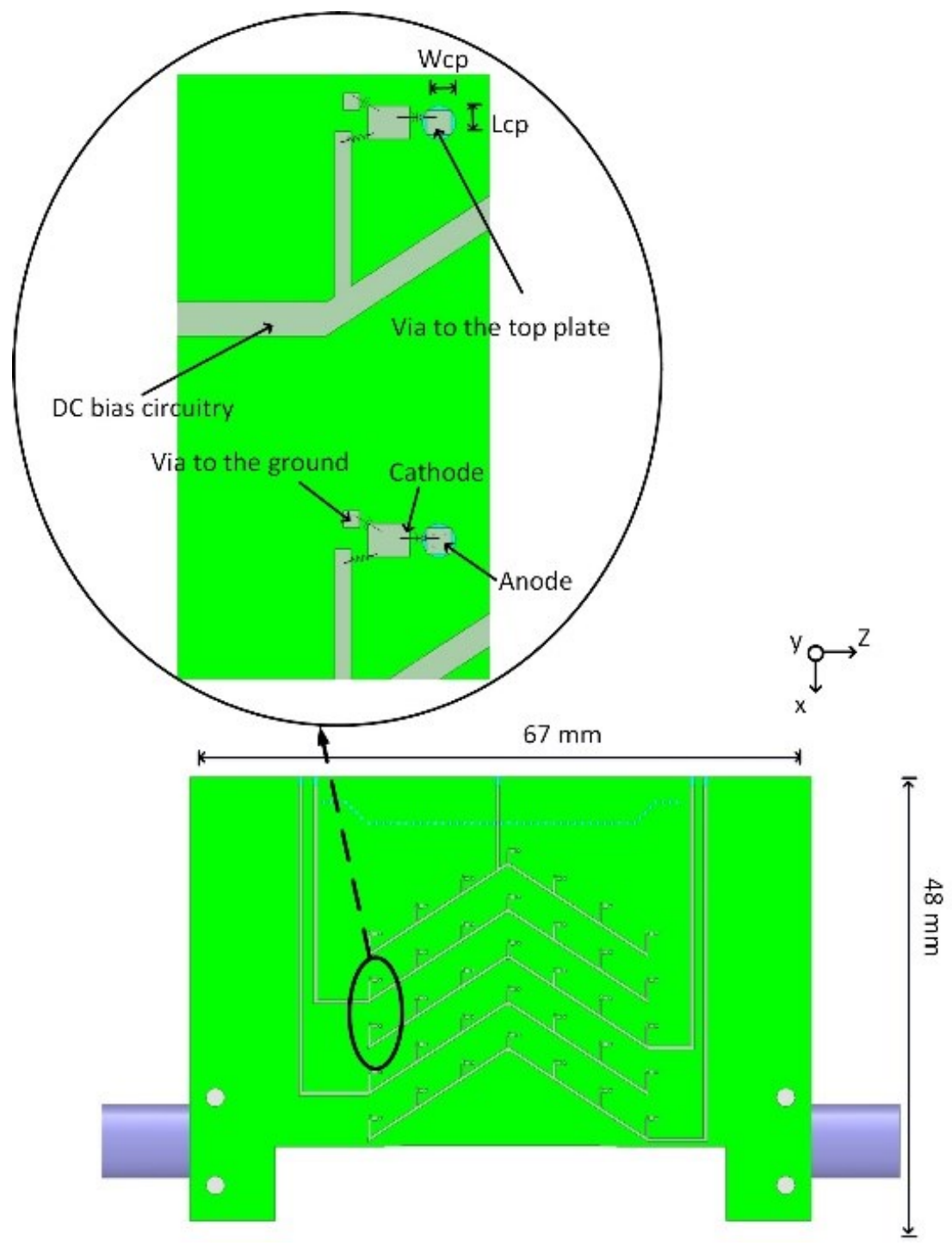

(a)

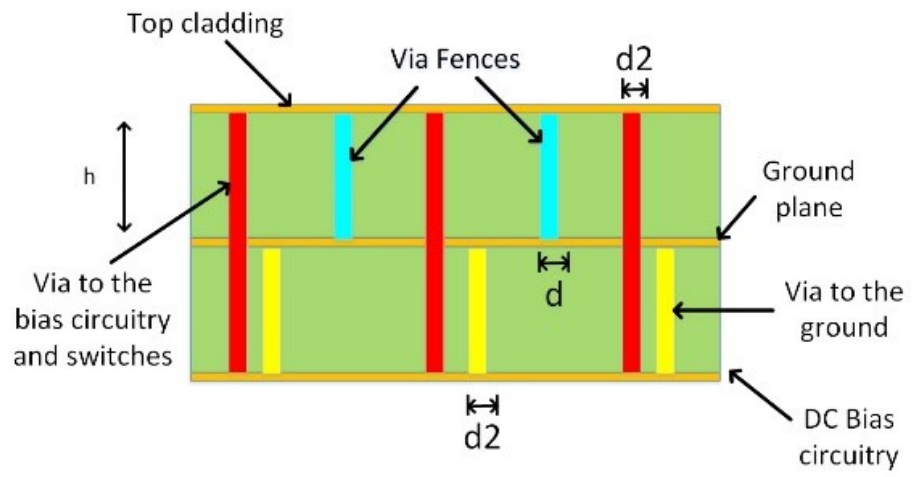

(b)

Figure 5.16. The proposed reconfigurable HMSIW LWA composed of circular slots in a V-shape configuration [103]. (a) Bottom view, (b) Side view. 


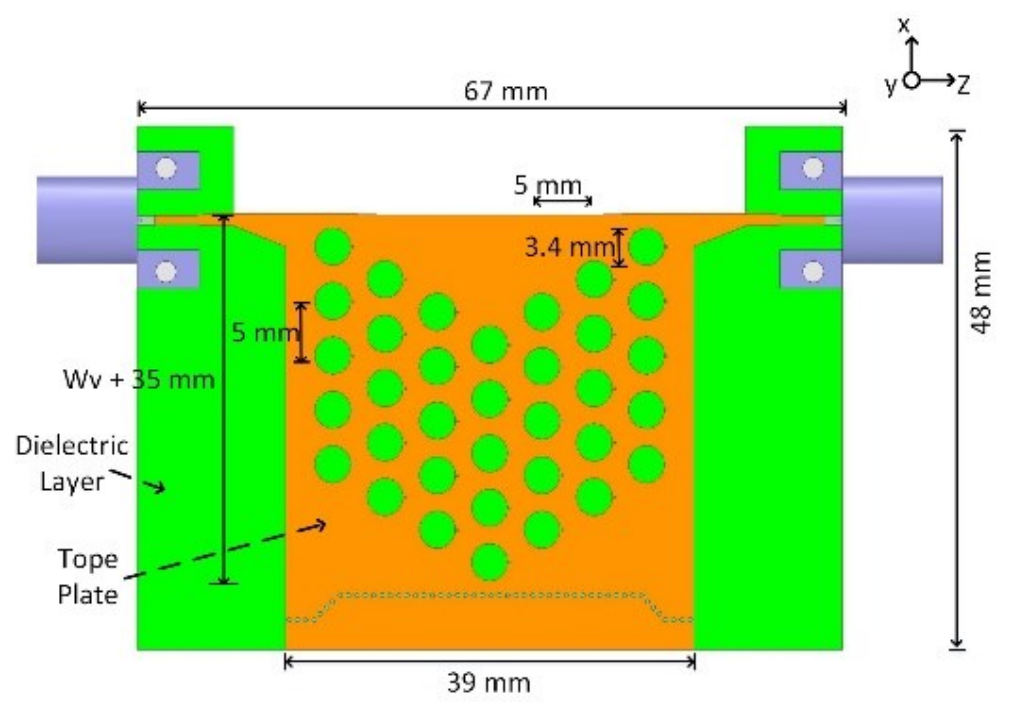

(c)

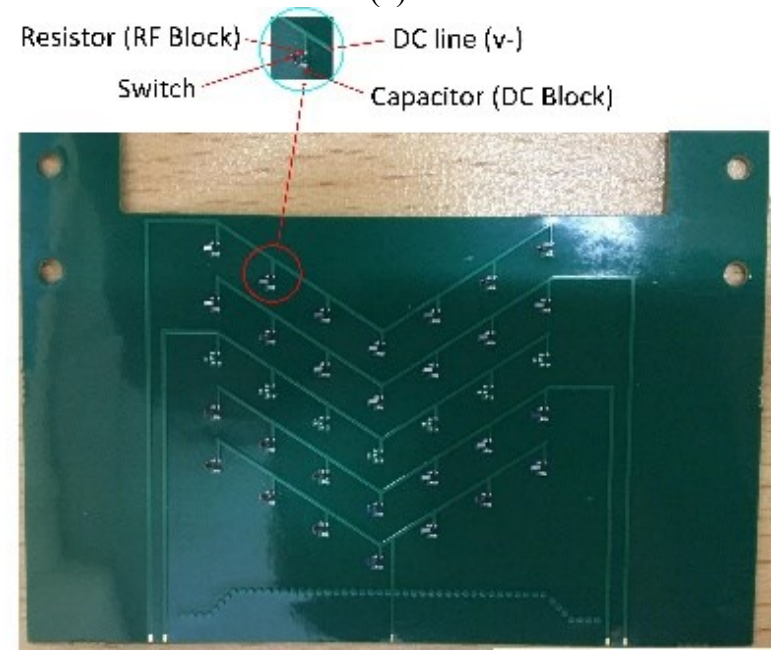

(d)

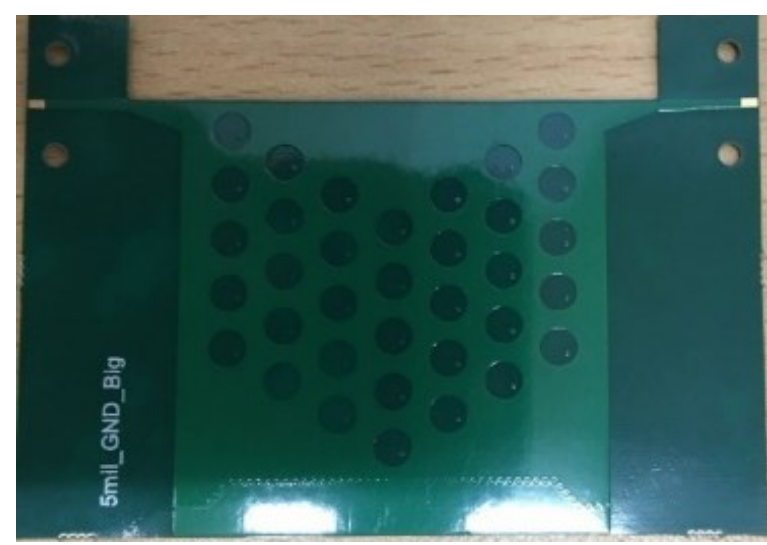

(e)

Figure 5.16. The proposed reconfigurable HMSIW LWA composed of circular slots in a V-shape configuration [103] (cont'd). (c) Top view, (d) Bottom view of the fabricated antenna, (e) Top view of the fabricated antenna. 
Table 5.2. Important geometrical parameters of the proposed reconfigurable HMSIW-based antenna [103].

\begin{tabular}{|c|c|c|c|c|c|c|}
\hline Parameter & $\mathrm{h}$ & $\mathrm{d}$ & $\mathrm{d}_{2}$ & $\mathrm{~W}_{\mathrm{v}}$ & $\mathrm{L}_{\mathrm{pc}}$ & $\mathrm{W}_{\mathrm{pc}}$ \\
\hline Value (mm) & 0.127 & 0.4 & 0.2 & 0 & 0.3 & 0.3 \\
\hline
\end{tabular}

The proposed antenna contains thirty-five circular cells in a V-shape configuration. Each reconfigurable cell is in the shape of a circle with a diameter of $3.4 \mathrm{~mm}$. The minimum size of the reconfigurable cell was dictated by the fabrication design rules, which required specific line width and spacing between components to accommodate the practical implementation. The circular shape leads to a more unfirm surface current distribution than the rectangular one due to removal of sharp corners. The locations, the number, and the period of the reconfigurable cells were determined through full-wave simulation in combination with physical intuition to achieve the optimum beam-scanning range along with small gain variation and adequate return loss. Overall, five V-shape rows containing thirty-five cells were implemented to achieve the desired performance while keeping the antenna compact. The electric field distributions of the proposed HMSIW with and without reconfigurable cells are presented in Fig. 5.17. Because of the large width of HMSIW and feeding through microstrip transitions and surface-mounted connectors, higher-order waveguide mode is excited, as shown in Fig. 5.17(a). The presence of multiple nodes in the field distribution confirms the excitation of the higherorder mode. Whereas in a narrow HMSIW supporting only the fundamental mode, as shown in Fig. 3.1, the maxima of the E-field only occur at the open side aperture. 


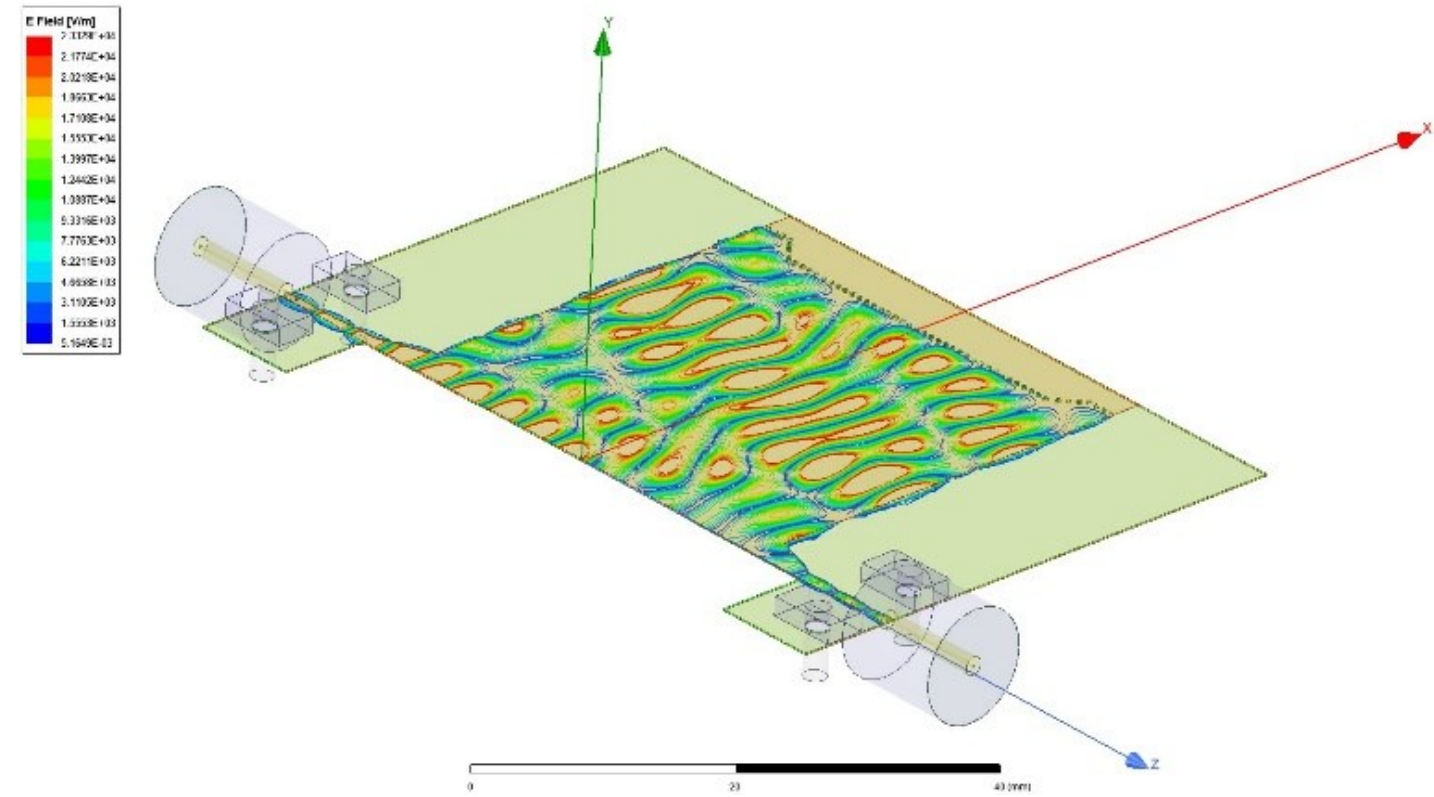

(a)

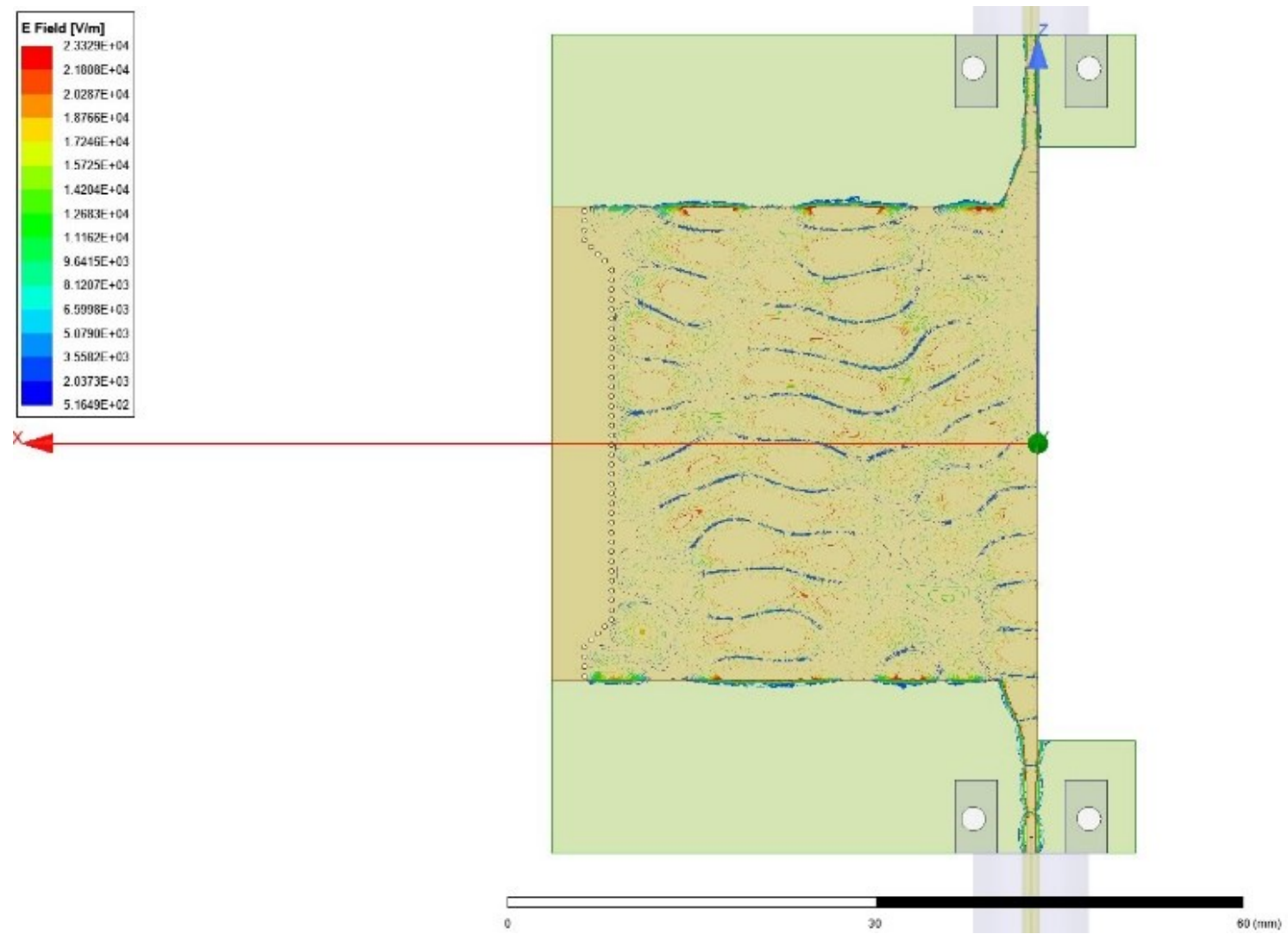

(b)

Figure 5.17. E-field distribution [103]. (a) Perspective view of HMSIW LWA without reconfigurable cells, (b) Top view of HMSIW LWA without reconfigurable cells. 


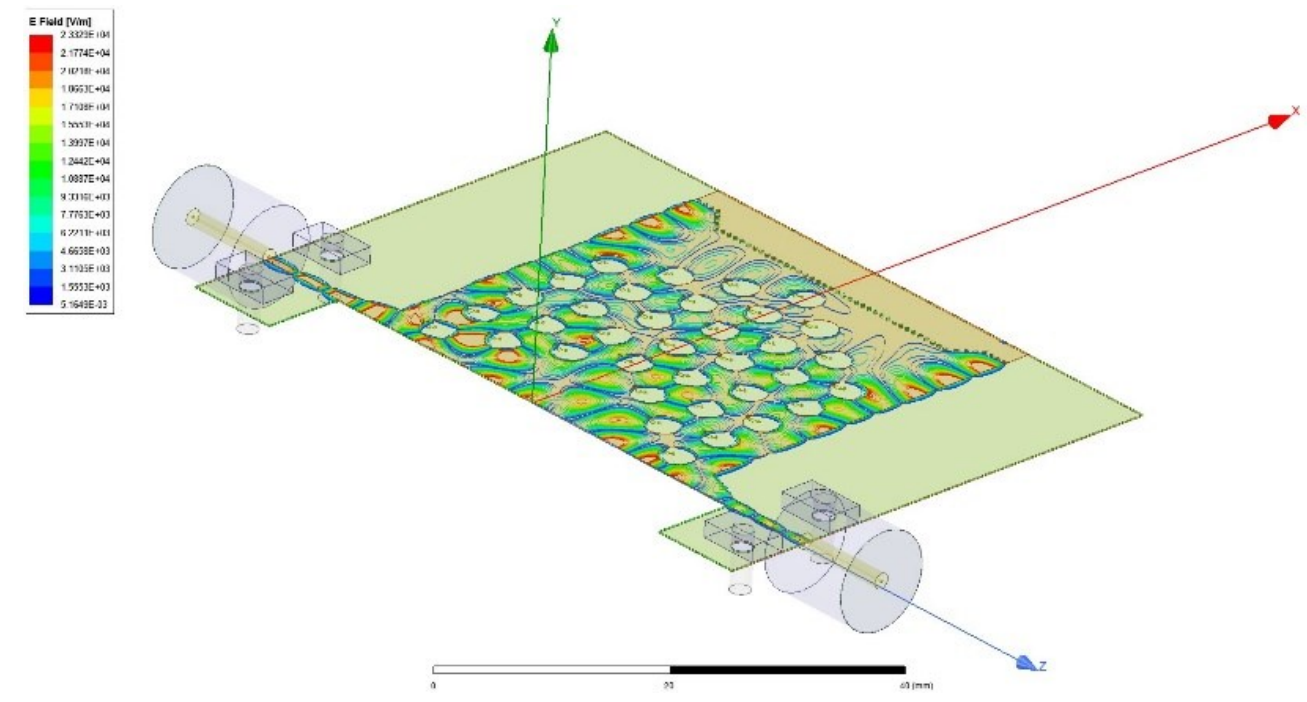

(c)

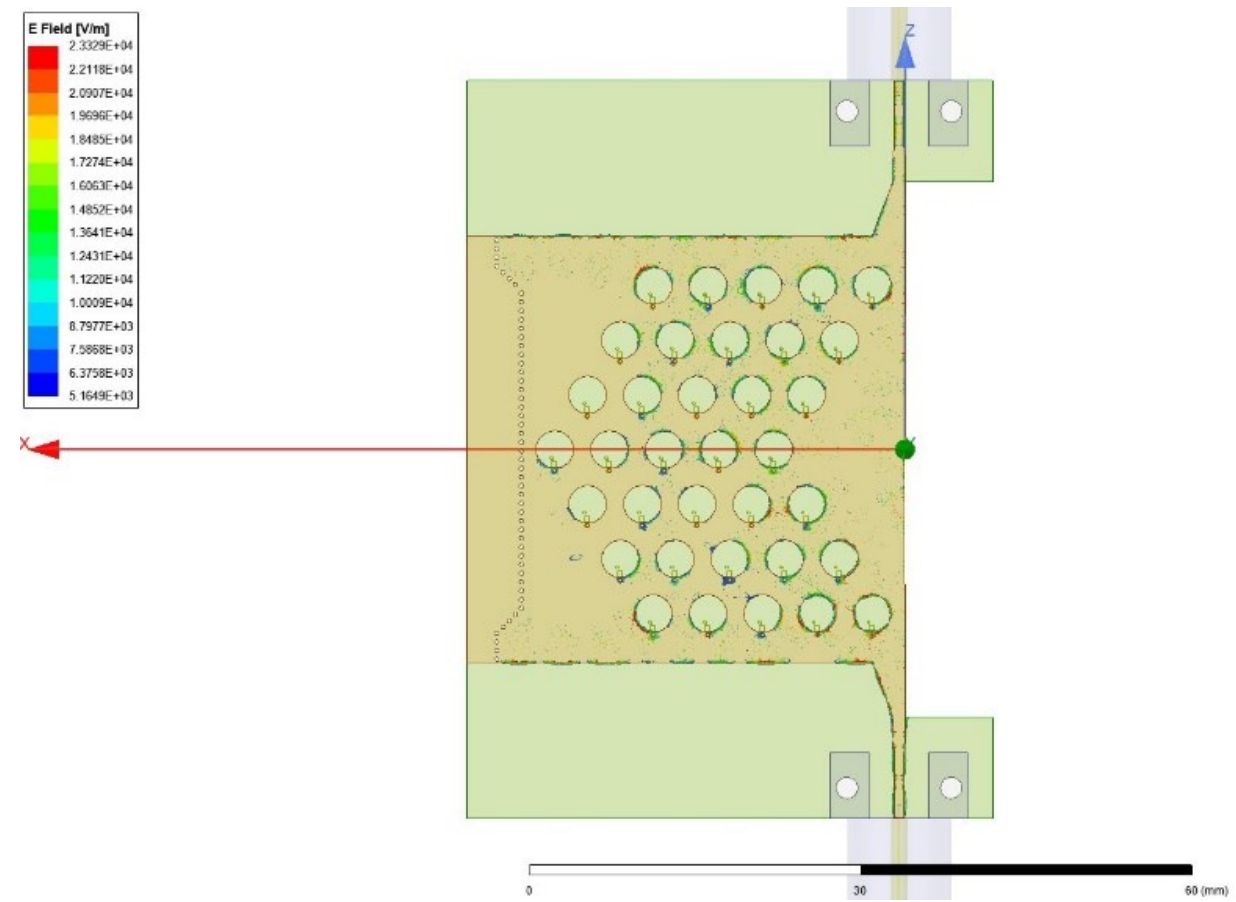

(d)

Figure 5.17. E-field distribution [103] (cont'd). (c) Perspective view of HMSIW LWA with circular slots in V-shape configuration, (d) Top view of HMSIW LWA with circular slots in V-shape configuration.

The reconfigurable cells were placed at the locations of the maximum electric field to perturb the field more effectively, as shown in Fig. 5.17(b). By implementing a V-shape configuration for the cells, transverse and longitudinal components of $\beta$ and the surface 
currents are disturbed by switching, leading to a relatively wide beam-scanning range. Placing the cells in a V-shape configuration improved the impedance matching as well.

The GaAs varactor diode MAVR-011020-14110P was used as the switch in each reconfigurable cell. According to the varactor diode datasheet [148], sweeping the reverse bias voltage in the range of $1-20 \mathrm{~V}$ leads to capacitance variations from 0.15 to $0.035 \mathrm{pF}$ at room temperature. The $\mathrm{Q}$ factor of the MAVR-011020-14110P varactor diode is 3000 at $4 \mathrm{~V}$ and $50 \mathrm{MHz}$ [148]. Furthermore, the ERA-1AEB102C chip resistor and GRM0335C1H4R7CA01D chip capacitor were used as RF and DC blocks in each reconfigurable cell, respectively. The anode was connected to the top plane through a via, while the cathode was connected to the bias circuitry through the RF block. The bias circuitry was placed underneath the antenna's ground plane to keep the structure compact, as shown in Fig. 5.16. The RF components were placed on the backside of the antenna to minimize their footprints' blockage effect on the radiation. The coupling between RF components and the top cladding was also reduced by placing them on the backside. Shrinking the reconfigurable cell size resulted in a more compact antenna. However, doing so increased the mutual coupling between the reconfigurable cells. The fabrication technology limitations also dictate the minimum cell size and period. The GaAs varactor diodes, DC blocks, and RF blocks were assembled on the pads using the ball grid array (BGA) method to minimize the inductance and resistance due to the assembly process. DC pads were connected to the DC source through sets of thin, flexible, and strong wires.

The full-wave simulation results were obtained using ANSYS HFSS v19.2. Similar to the previous measurement setups, S-parameters were measured using a Rohde \& Schwarz 
ZVA-67 VNA, and radiation patterns were measured at an anechoic chamber using MI12A-26 as a standard gain horn antenna. The measurement setup is shown in Fig. 5.18.

Sweeping the varactor diodes bias voltage results in the variation of the series capacitance of the structure, which changes the series resonance frequency and the input impedance. This leads to variations of $\beta$ and $\theta_{0}$ according to (5.1) and (2.3). In the proposed HMSIW LWA, all switches in a V-shape row were biased similarly to simplify the bias circuitry. Four switching states are reported as follows to validate the electronic beam-scanning capability; (1) all switches are biased at $0 \mathrm{~V}$ (i.e., open circuit), (2) the first two rows (i.e., closest ones to the open side aperture) are biased at $4 \mathrm{~V}$ and the rests are biased at $0 \mathrm{~V},(3)$ the first two rows are biased at $15 \mathrm{~V}$ and the rests are at $0 \mathrm{~V}$, and (4) all rows are biased at $1 \mathrm{~V}$. Unfortunately, due to the error in the antenna's fabrication, few switches in the last V-shape row (i.e., closest to the side via fence) were short-circuited. Hence, the last row was not connected to the DC voltage to protect the power source. This resulted in deviations from the initial simulated results. In the final simulated results, the last row was considered defective to capture the reality.

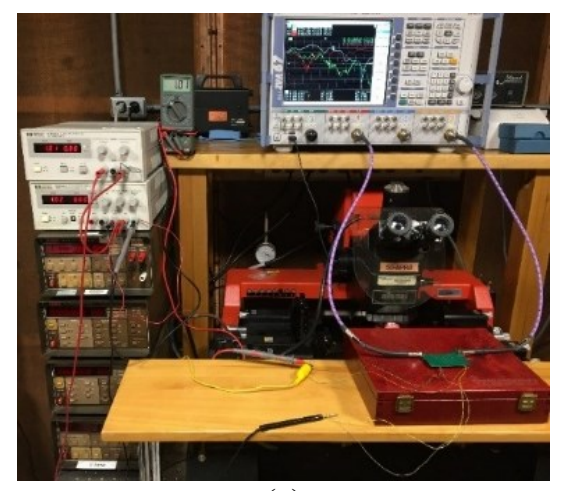

(a)

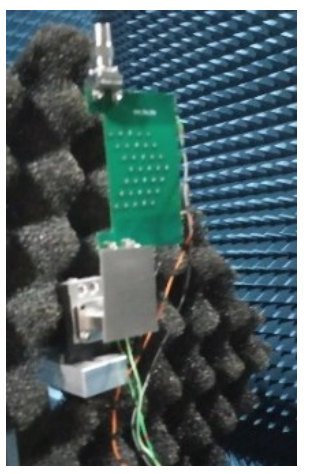

(b)

Figure 5.18. Measurement setup [103]. (a) S-parameters measurement setup, (b) Antenna under test in the anechoic chamber for measuring radiation pattern. 
The measured and simulated S-parameters for different switching states are presented in Fig. 5.19. Return loss is better than $10 \mathrm{~dB}$ over the frequency range of $28-29.5 \mathrm{GHz}$ in all different switching states. Since the implemented varactor diodes have constant return loss [148], there is little variation in the return loss by sweeping the reverse bias voltage. The measured insertion loss ranges from 12 to $20 \mathrm{~dB}$ for different switching states over the frequency band of $27-30 \mathrm{GHz}$, which is partly because of the losses of the varactor diodes and RF blocks. The other loss is due to the thin substrate implemented to achieve a compact structure. This contributed to high conduction loss, low gain, and high insertion loss.

The measured and simulated radiation patterns for four different switching states in Eplane (YZ-plane) at $28 \mathrm{GHz}, 28.5 \mathrm{GHz}$, and $29 \mathrm{GHz}$ are presented in Fig. 5.20, confirming the electronic beam-scanning capability of the proposed LWA. As stated before, E-plane is at YZ-plane. In contrast, the H-plane is at different $\theta$ planes since the main-lobe shifts by switching. As for other antennas investigated in this dissertation, only the radiation pattern in the E-plane was investigated since measuring the H-plane radiation pattern was out of the capability of the measurement system. Figs. 5.20(e)-(h) indicates that the antenna's main-lobe scans $29^{\circ}$ by switching at $28.5 \mathrm{GHz}$. As observed in Fig. 5.20, the antenna radiates backward (i.e., $90^{\circ}<\theta_{0}<180^{\circ}$ ), which is due to the propagation of the higher-order waveguide modes. Backward beam-scanning can be used for monitoring the blind-spot of an autonomous vehicle. Although the proposed antenna is designed for electronic beam-scanning applications, the main-lobe pointing angle changes by sweeping the frequency. This is due to the antenna's leaky-wave nature. 
According to Fig. 5.20, the measured and the simulated frequency beam-squint in the frequency band of $27-30 \mathrm{GHz}$ are $18^{\circ}$ and $22^{\circ}$, respectively.

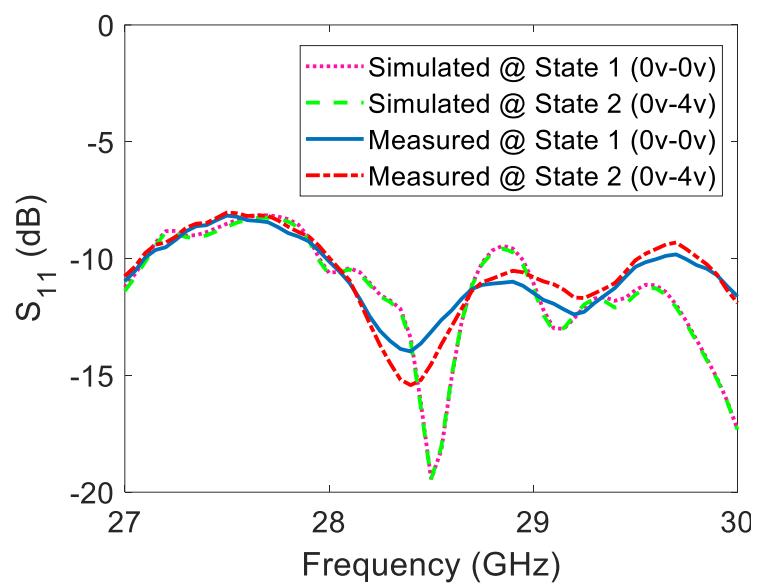

(a)

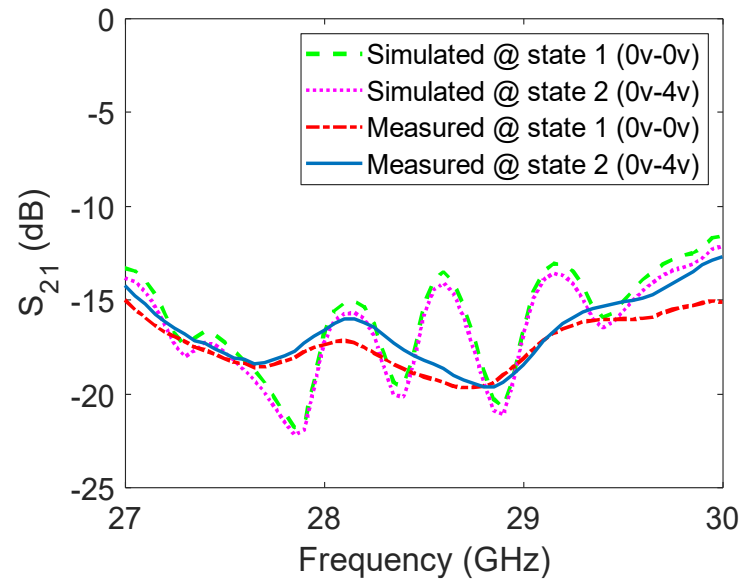

(c)

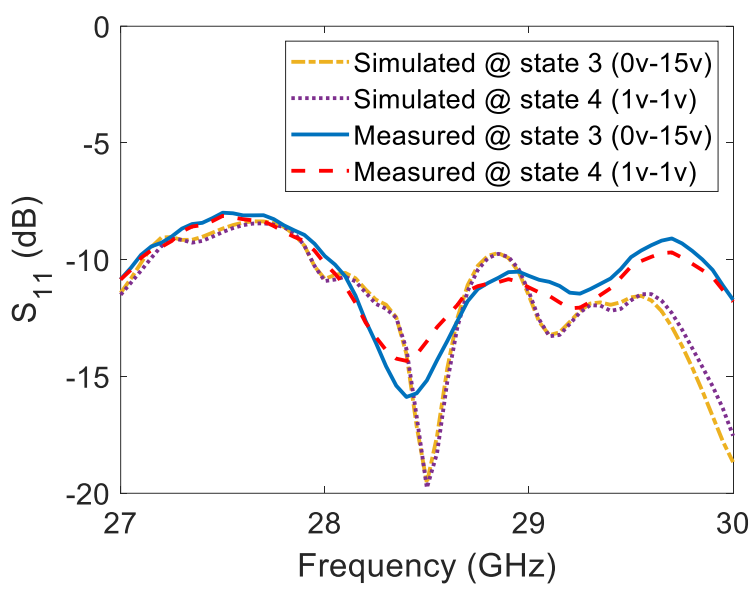

(b)

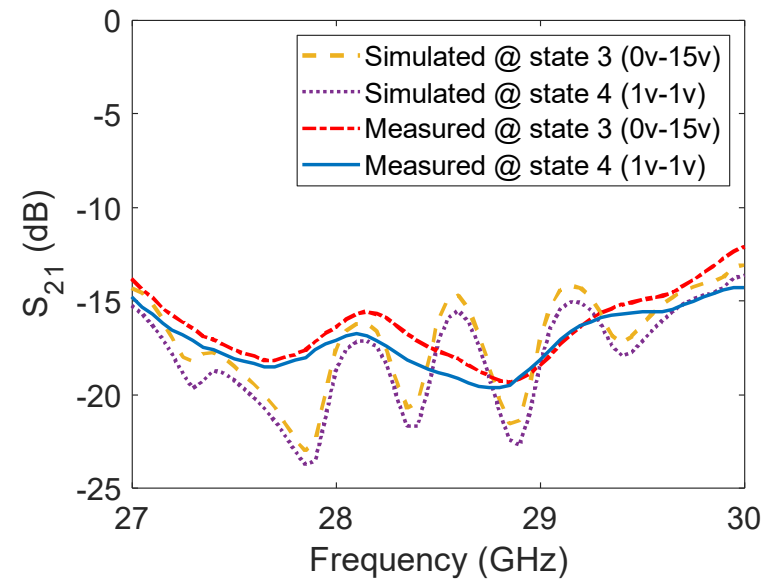

(d)

Figure 5.19. S-parameters of the reconfigurable HMSIW LWA with reconfigurable cells in V-shape configuration [103]. (a) Return loss at states 1 and 2, (b) Return loss at states 3 and 4, (c) Insertion loss at states 1 and 2, and (d) Insertion loss at states 3 and 4. 


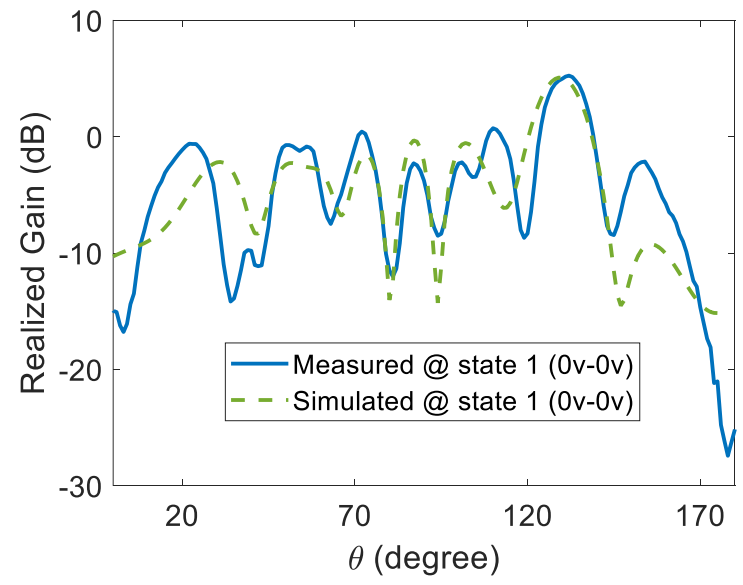

(a)

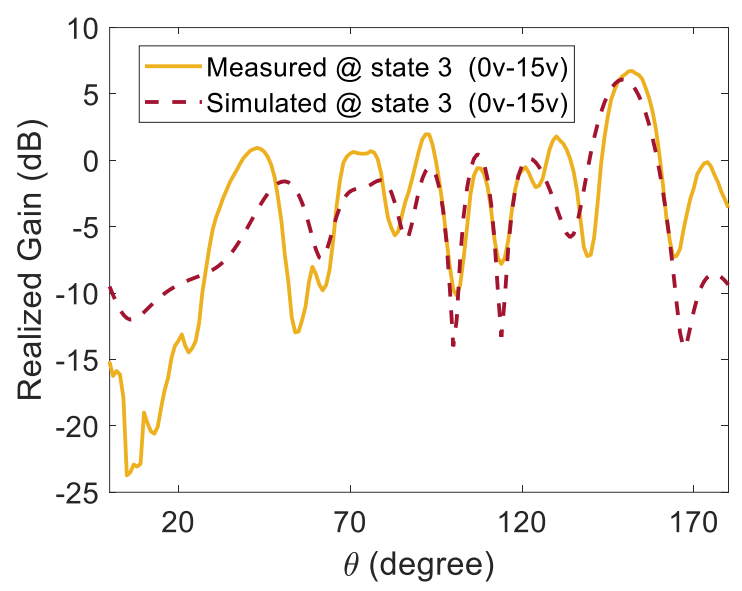

(c)

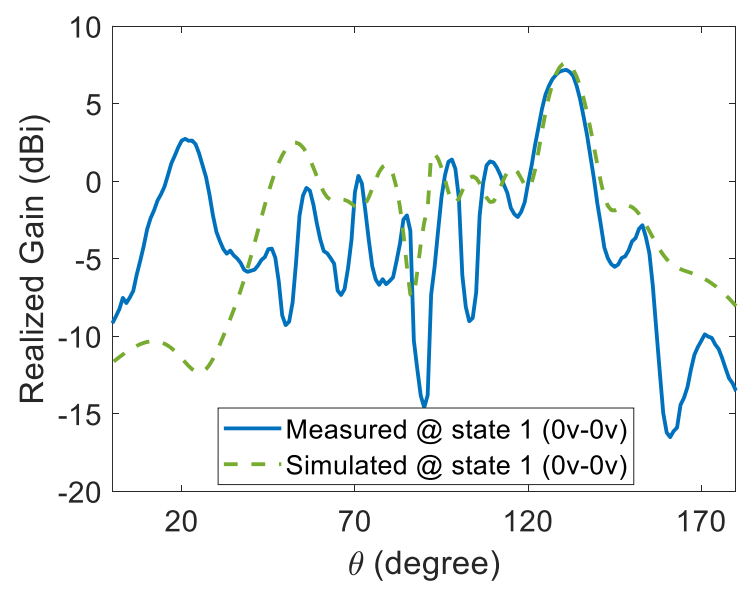

(e)

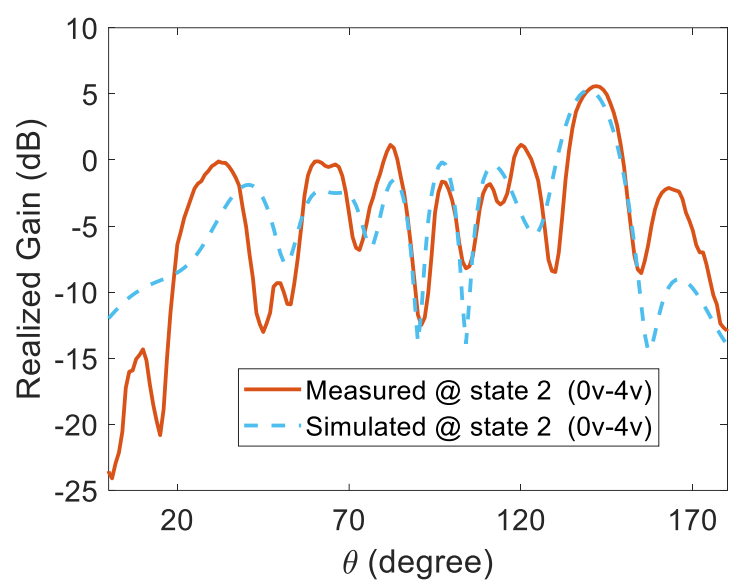

(b)

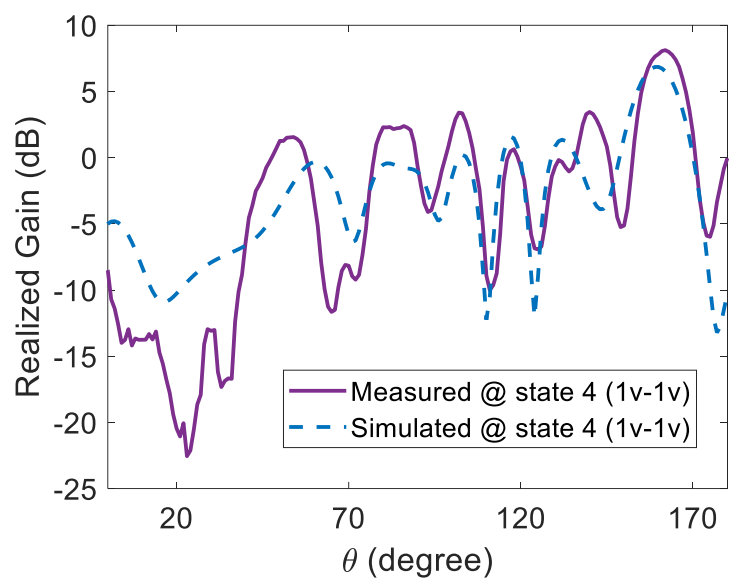

(d)

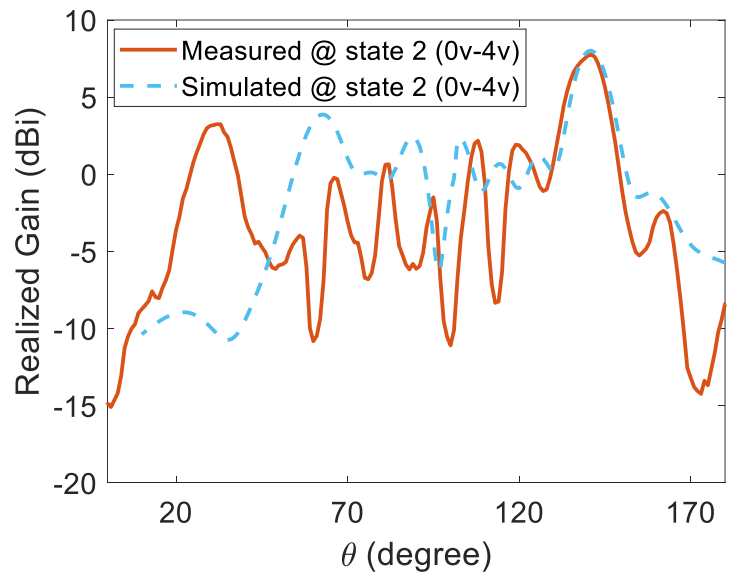

(f)

Figure 5.20. Gain radiation patterns of the proposed reconfigurable HMSIW LWA in E-plane (YZplane) for different switching states and frequencies [103]. (a) State 1 at $28 \mathrm{GHz}$, (b) State 2 at 28 GHz, (c) State 3 at $28 \mathrm{GHz}$, (d) State 4 at $28 \mathrm{GHz}$, (e) State 1 at $28.5 \mathrm{GHz}$, (f) State 2 at $28.5 \mathrm{GHz}$. 


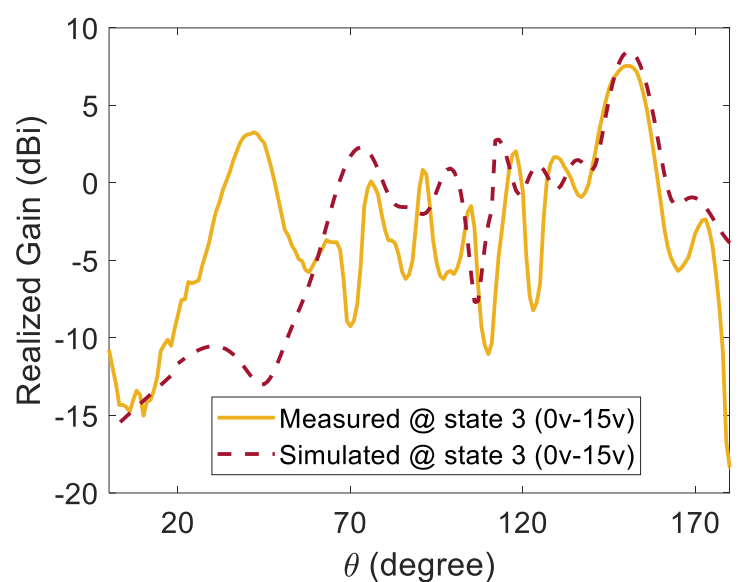

$(\mathrm{g})$

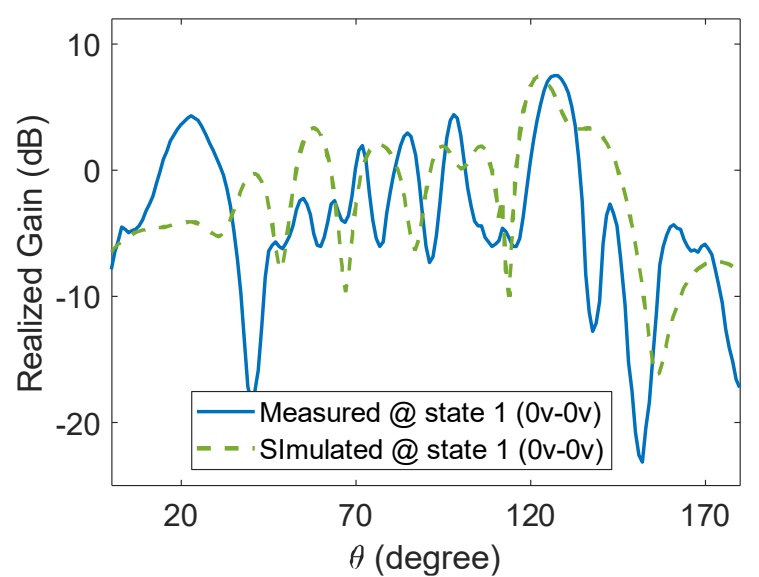

(i)

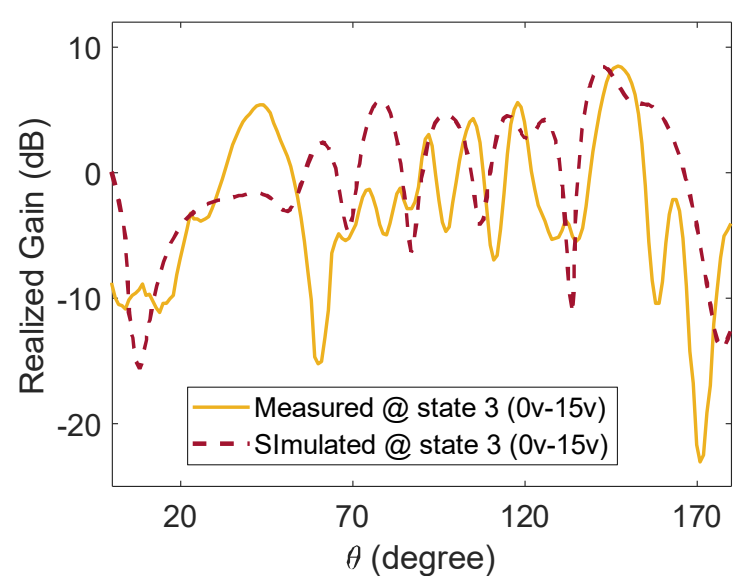

(k)

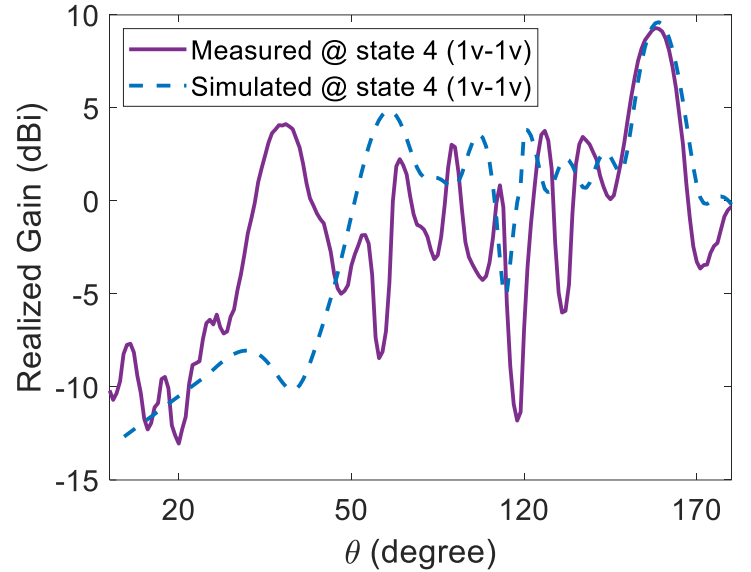

(h)

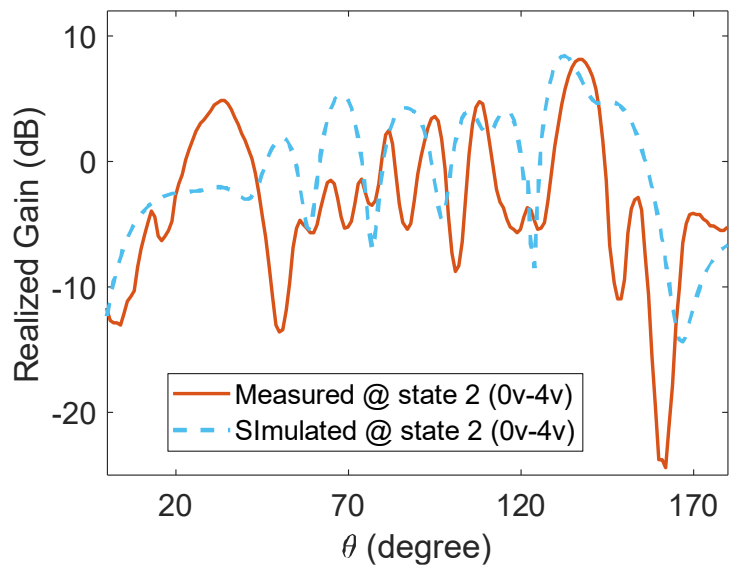

(j)

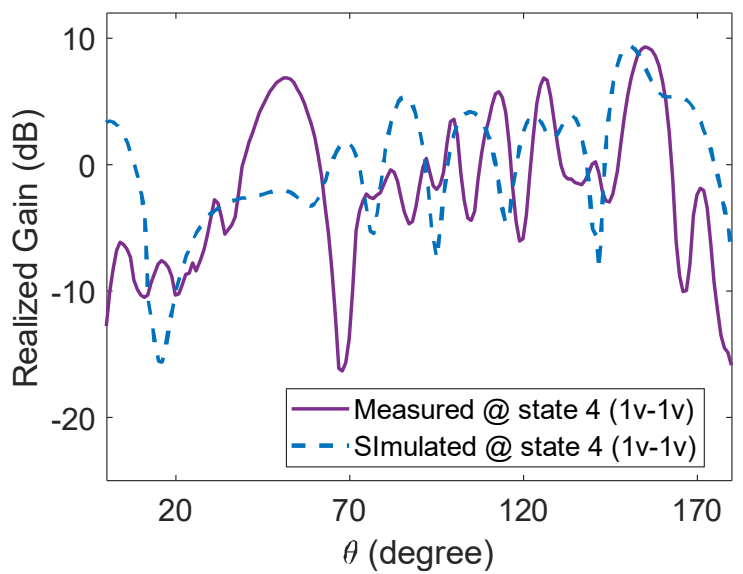

(l)

Figure 5.20. Gain radiation patterns of the proposed reconfigurable HMSIW LWA in E-plane (YZplane) for different switching states and frequencies [103] (cont'd). (g) State 3 at $28.5 \mathrm{GHz}$, (h) State 4 at $28.5 \mathrm{GHz}$, (i) State 1 at $29 \mathrm{GHz}$, (j) State 2 at $29 \mathrm{GHz}$, (k) State 3 at $29 \mathrm{GHz}$, and (l) State 4 at 29

GHz. 
The peak realized gain for different switching states in the frequency band of 27-30 $\mathrm{GHz}$ are reported in Fig. 5.21, indicating that switching results in $1.2 \mathrm{dBi}$ gain variation at $28.5 \mathrm{GHz}$. Hence, the proposed structure scans space with small gain variation at the desired frequency, which is beneficial in beam-scanning applications. According to Fig. 5.21 , the measured peak realized gain varies from $-2.5 \mathrm{dBi}$ to $10.6 \mathrm{dBi}$ over the frequency band of $27-30 \mathrm{GHz}$ for different switching states.

As discussed in Chapters 2 and 3, the SLL can be reduced by tapering the side via fence [64]-[66] or the side aperture [69], [70]. It should be noted that the high SLL is due to the small thickness of the board [63] and undesired reflection from the side aperture. Increasing the thickness of a conventional narrow-width HMSIW, supporting the fundamental mode, may lead to the SLL improvement [63]. However, this trend was not observed in our case, which can be attributed to the higher-order mode excitation. Realizing the proposed design on a thicker substrate can improve the gain by reducing the conductor loss and improving impedance matching. However, this is achieved at the expense of compromising the compact nature of the antenna. The gain can also be increased by increasing the number of cells at the expense of the cost and size of the antenna. Biasing the switches non-uniformly provides more switching states and results in more variations in the phase constant. Hence, achieving a wider beam-scanning range will be possible at the expense of increasing the feed network's complexity and cost. Increasing the variable capacitance range of the switches also increases the beamscanning range. However, achieving a high speed and low loss switch with a wide variable capacitance range at the mm-wave band is a practical challenge. 


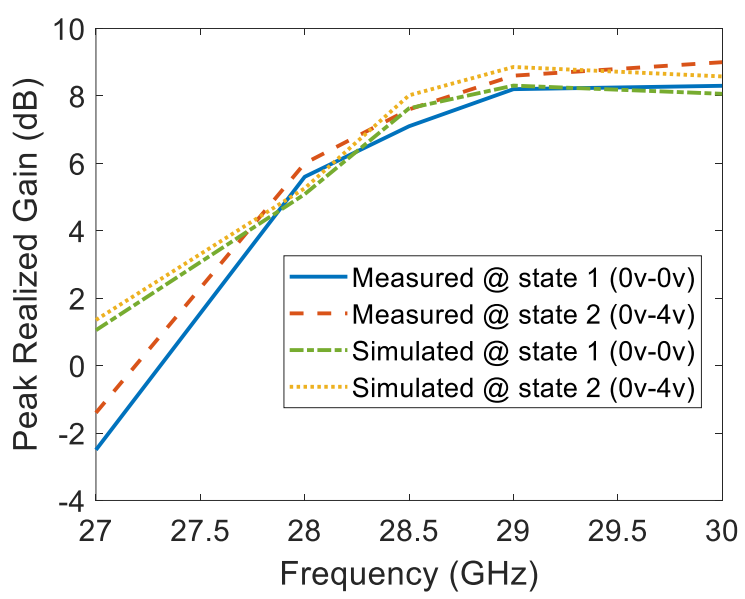

(a)

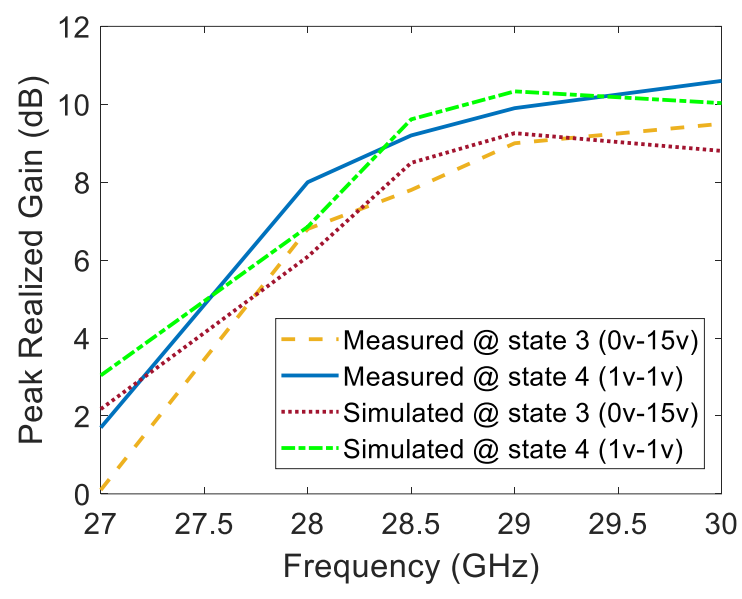

(b)

Figure 5.21. Peak realized gain of the proposed reconfigurable HMSIW LWA [103]. (a) States 1 and

\section{2, (b) States 3 and 4.}

A sensitivity analysis was performed to investigate the discrepancies between simulated and measured results more thoroughly. Therefore, several parameters such as board's thickness $(h)$, vias size ( $d$ and $\left.d_{2}\right)$, extension width of the waveguide section $\left(W_{v}\right)$, and pad sizes $\left(L_{p c}\right.$ and $\left.W_{p c}\right)$ were changed. S-parameters and radiation patterns for different combinations are demonstrated in Figs. 5.22 and 5.23. According to the sensitivity analysis, $h$ and $W_{v}$ contribute the most to the discrepancies between measured and simulated results.

As for other antennas investigated in this dissertation, the proposed antenna may bend under physical stress because of the relatively small thickness of the substrate. This is a real concern and a possible reason for the discrepancies between the measured and the simulated results, which need to be investigated. To investigate this case, the antenna was gradually bent such that the vertical distance from the horizon changed up to $h_{b}=5.6 \mathrm{~mm}$ by moving toward the two ends, as shown in Fig. 5.24. The S-parameters, normalized radiation patterns, and peak realized gain of the bent reconfigurable HMSIW LWA are 
demonstrated in Figs. 5.25-5.27 confirming the large degradations in the antenna performance in terms of return loss, peak realized gain, SLL, $\Delta \theta$, and $\theta_{0}$.

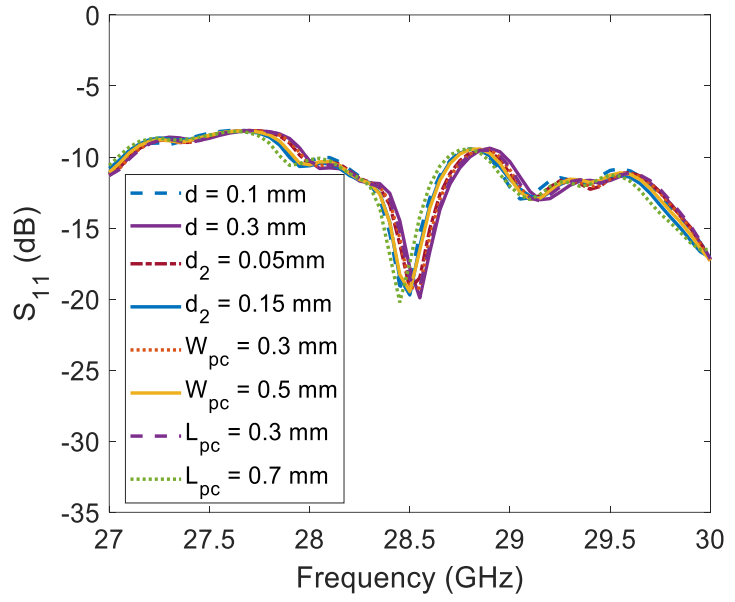

(a)

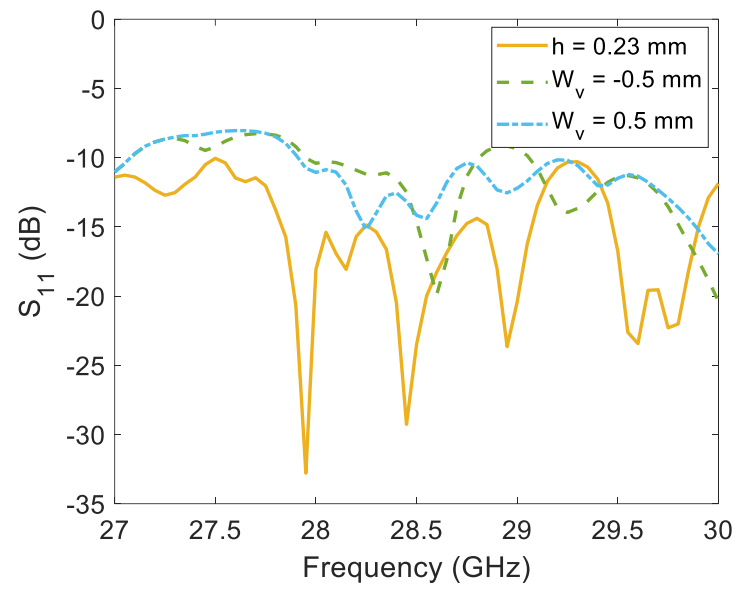

(c)

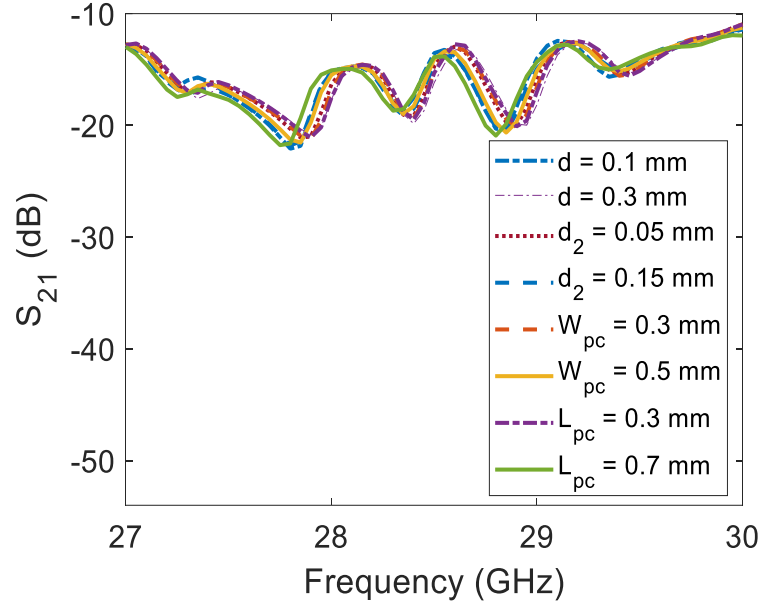

(b)

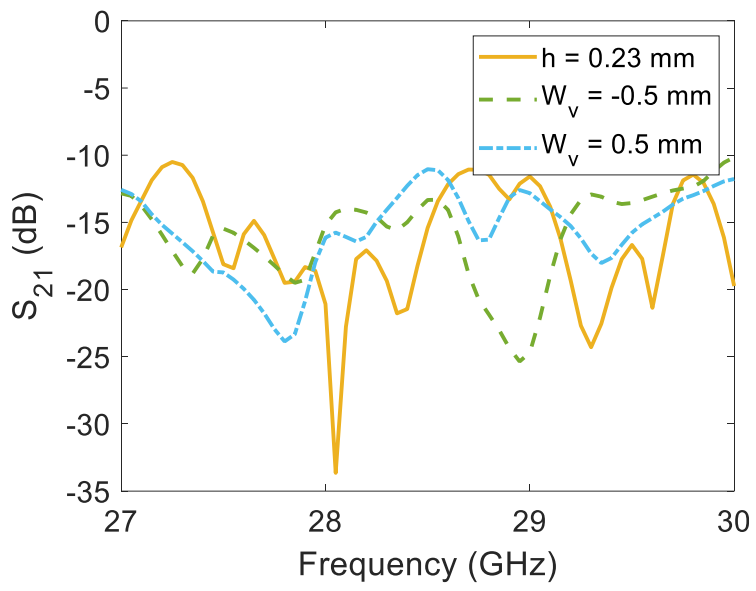

(d)

Figure 5.22. S-parameters for different geometrical parameters at state 2 [103]. (a) $S_{11}$ for different values of $d, d_{2}, W_{p c}$, and $L_{p c}$, (b) $S_{21}$ for different values of $d, d_{2}, W_{p c}$, and $L_{p c}$, (c) $S_{11}$ for different values of $h$ and $W_{v}$, (d) $S_{21}$ for different values of $h$ and $W_{v}$. 


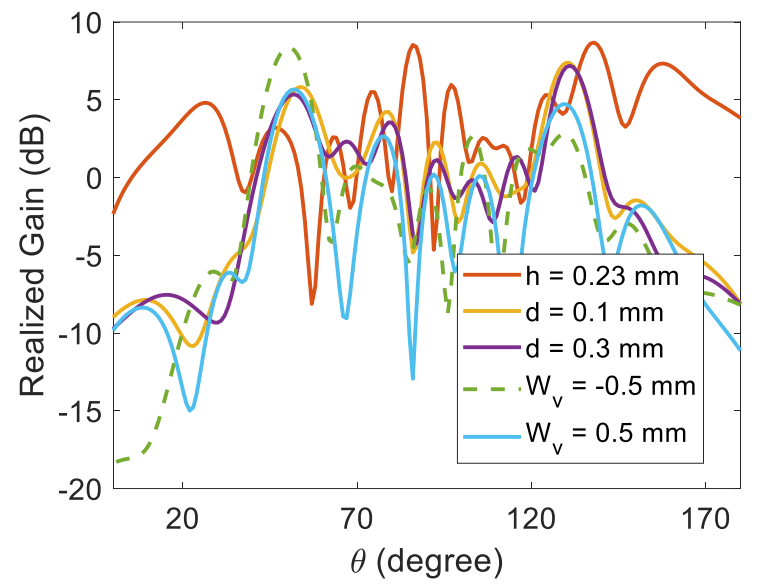

(a)

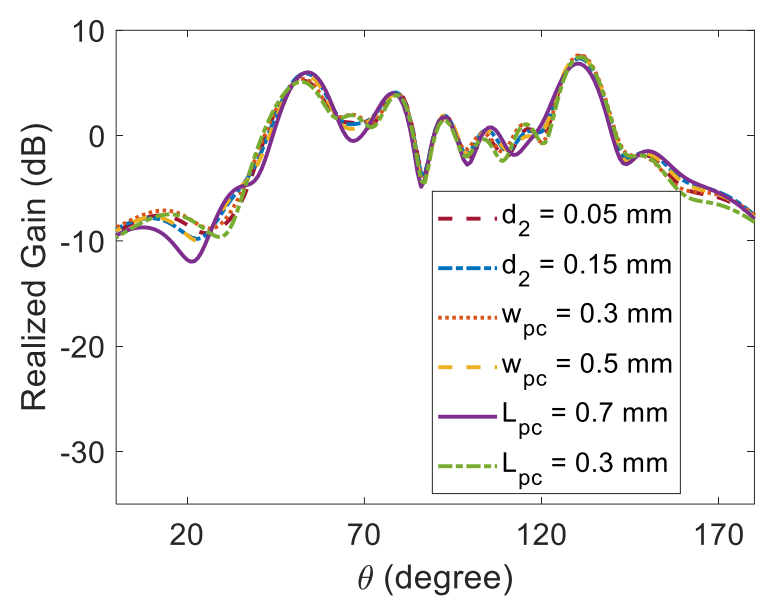

(b)

Figure 5.23. Radiation patterns at state 2 for different geometrical configurations [103]. (a) Different values of $h, d$, and $W_{v}$, (b) Different values of $d_{2}, W_{p c}$, and $L_{p c}$.

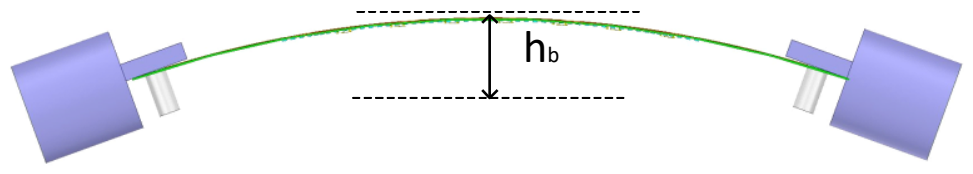

(a)

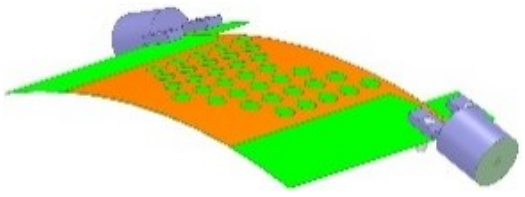

(b)

Figure 5.24. Schematic view of the bent reconfigurable HMSIW LWA [103]. (a) Side view, (b) 3D view.

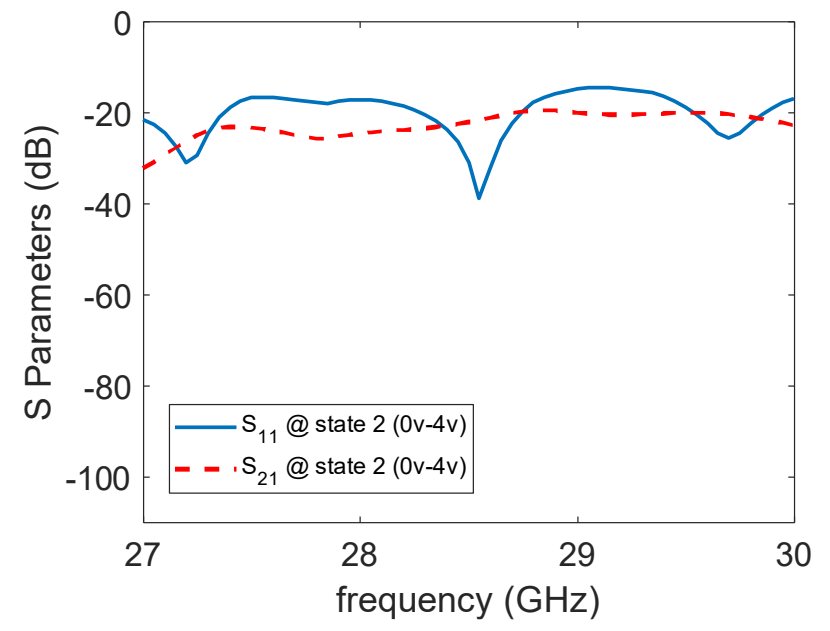

Figure 5.25. S-parameters of the bent reconfigurable HMSIW LWA [103]. 


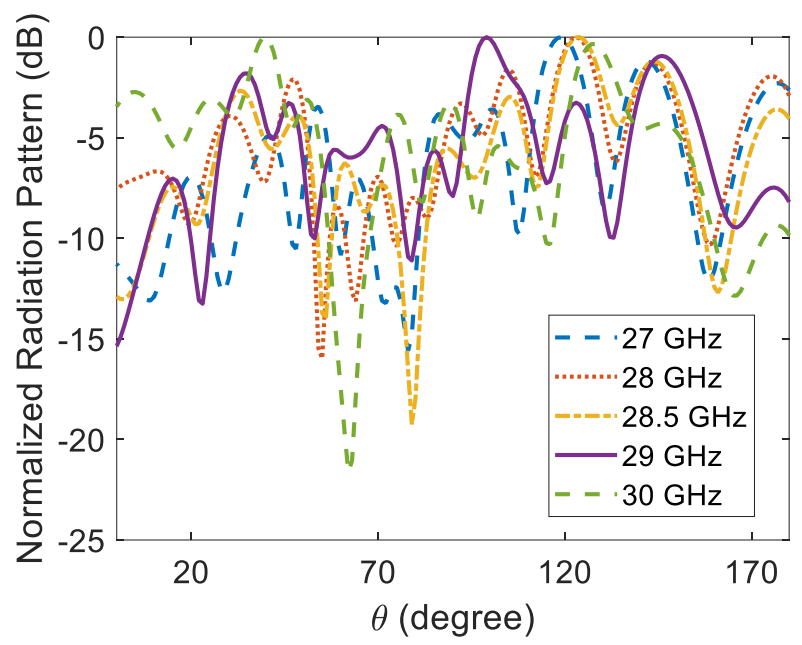

Figure 5.26. Normalized radiation patterns of the bent reconfigurable HMSIW LWA at switching state 2 [103].

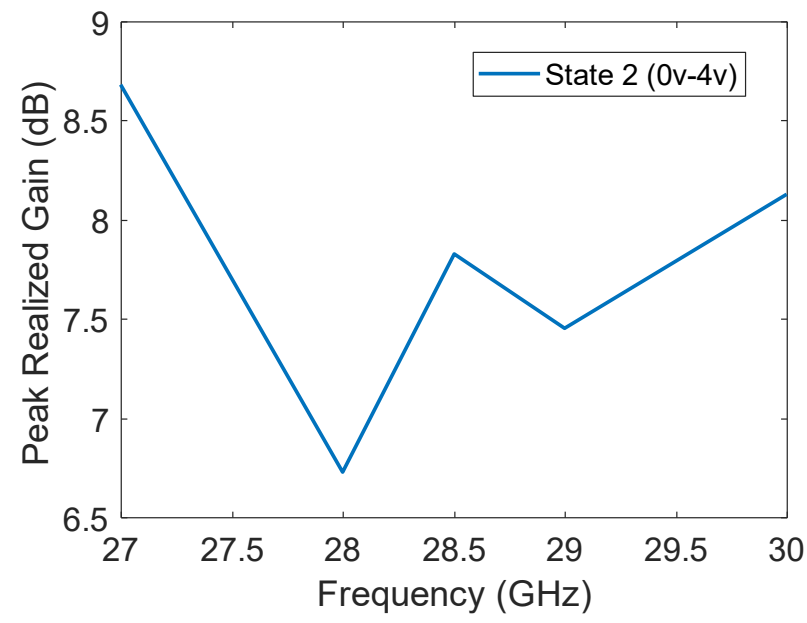

Figure 5.27. Peak realized gain of the bent reconfigurable HMSIW LWA at switching state 2 [103].

A comparison with similar electronic beam-scanning antennas in [94]-[99] is shown in Table 5.3. A key difference is that the reported operating frequency bands of [95]-[99] are lower than the antenna proposed here for $5 \mathrm{G}$ mm-wave applications. It should be noted that by increasing the frequency, both the loss and the parasitic effects of electronic components increase. Therefore, the antennas' performance reported in [95]-[99] may degrade if the same designs are scaled to operate in mm-wave frequency band.

The beam-scanning range of the antenna proposed here is wider than the reported ones in [94]-[96] and comparable to [97]. The wider beam-scanning range of [98] and [99] are 
due to the different methods that were applied to achieve the electronic beam-scanning. The reconfigurable cells of the proposed design were integrated into HMSIW, while cells were added as extra stubs to the HMSIW in [94]-[99]. The beam-scanning in [99] was achieved using two sets of series and parallel switches, while only one switch per cell was used in the design investigated in this section.

The beam-scanning range should be considered in light of the gain variation. The most stringent gain variation (i.e., $\pm 0.6 \mathrm{dBi}$ ) was chosen as the baseline to define the beamscanning range in the proposed design here. Overall, the gain variation of the reconfigurable antenna proposed here is smaller than the ones in [94]-[96] and [99], and comparable to [97] and [98]. This is beneficial because the gain variation in beamscanning applications should be minimized. Overall, a more generous allowance of the gain variation would lead to an over-optimistic estimate of the beam-scanning range.

As stated before, the electronic components were located on the backside of our proposed antenna. This is another distinction of the design proposed here as compared to [94]-[99]. This led to the ease of the fabrication and assembly due to the larger available space on the backside. Moreover, the main-beam of the proposed antenna is pointed backward, which is useful for monitoring the blind-spot in an autonomous vehicle.

The radiating length of the antenna investigated here is smaller than the ones reported in [94]-[97]. This is advantageous since small size is a premium in beam-scanning applications. The higher peak gain of the antennas presented in [94]-[97] are due to their longer electric length. The smaller thickness of the proposed structure leads to higher conductor loss and smaller gain than [98] and [99]. This is the cost of achieving a lowprofile antenna. 
Table 5.3. Comparison among the similar reported electronic beam-scanning antennas [103].

\begin{tabular}{|c|c|c|c|c|}
\hline Design & Scanning Range & Peak Gain (dBi) & Center Frequency & Radiating Length \\
\hline $\begin{array}{c}\text { Our proposed } \\
\text { reconfigurable } \\
\text { HMSIW LWA }\end{array}$ & $29^{\circ}$ & $8.2 \pm 0.6$ & $28.5 \mathrm{GHz}$ & $3.7 \lambda$ \\
\hline$[94]$ & $21^{\circ}$ & $12 \pm 1.5$ & $24 \mathrm{GHZ}$ & $6.7 \lambda$ \\
\hline$[95]$ & $23^{\circ}$ & $13 \pm 0.84$ & $10.8 \mathrm{GHZ}$ & $7.6 \lambda$ \\
\hline$[96]$ & $16^{\circ}$ & $10 \pm 2$ & $6.5 \mathrm{GHZ}$ & $5.36 \lambda$ \\
\hline$[97]$ & $29^{\circ}$ & $12.9 \pm 0.6$ & $6 \mathrm{GHZ}$ & $5.32 \lambda$ \\
\hline$[98]$ & $39^{\circ}$ & $10.47 \pm 0.65$ & $4.2 \mathrm{GHZ}$ & $3 \lambda$ \\
\hline$[99]$ & $66^{\circ}$ & $9.55 \pm 1.75$ & $6.5 \mathrm{GHZ}$ & $3.25 \lambda$ \\
\hline
\end{tabular}

Overall, the proposed antenna's beam scans $29^{\circ}$ of space with a peak realized gain of $8.2 \pm 0.6 \mathrm{dBi}$ by switching between different states at $28.5 \mathrm{GHz}$ [103]. The small gain variations, operation in the mm-wave frequency band, compactness, backward beamscanning with adequate scanning range make the proposed antenna a suitable candidate for mm-wave $5 \mathrm{G}$ blind-spot monitoring and $\mathrm{V} 2 \mathrm{X}$ communications.

\subsubsection{Reconfigurable SIW LWA with Transverse Slots}

In the proposed reconfigurable antenna, beam-scanning is achieved by introducing sets of varactor diodes over transverse slots. Each reconfigurable cell is in the shape of a narrow rectangular slot containing varactor diode, Dc block, and RF block. The narrow rectangular shape slot was chosen due to its adequate control over the leakage. The proposed structure containing two Rogers RT/Duroid 5880 boards, each $0.127 \mathrm{~mm}$ thick is presented in Fig. 5.28. The total length, width, and thickness of the antenna are $68 \mathrm{~mm}$, $24 \mathrm{~mm}$, and $0.3 \mathrm{~mm}$, respectively. To further improve the impedance matching, three additional tapered slots were placed at each end. The radiation section of the antenna contains thirty rectangular transversal slots along a length of $29 \mathrm{~mm}$ obtained from (2.4). The length and width of the slots are $2 \mathrm{~mm}$ and $0.3 \mathrm{~mm}$, respectively to achieve an 
adequate control over the leakage. The slots were placed at a $1 \mathrm{~mm}$ distance from each other to prevent the excitation of higher order Floquet modes. To only allow $\mathrm{TE}_{10}$ to propagate, the via fences were set at a $4.5 \mathrm{~mm}$ distance transversally. Switching can be done uniformly or non-uniformly. First, the results of uniform switching are investigated [112]. Then the results of non-uniform switching are presented [113]. The full-wave simulations were carried out using Ansys HFSS v.19.2.

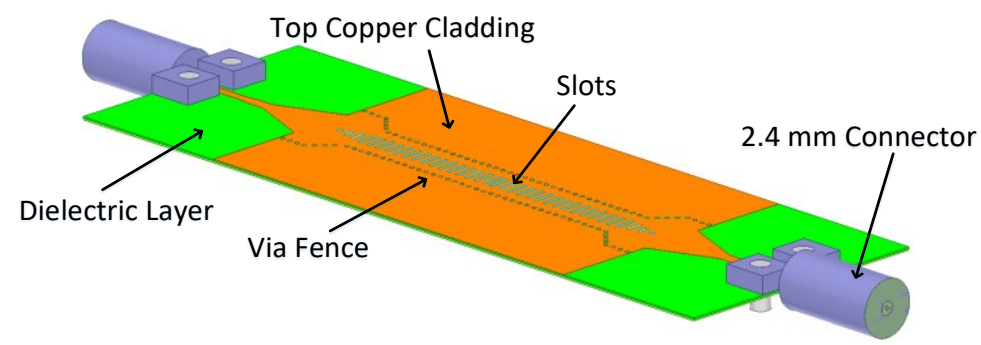

(a)

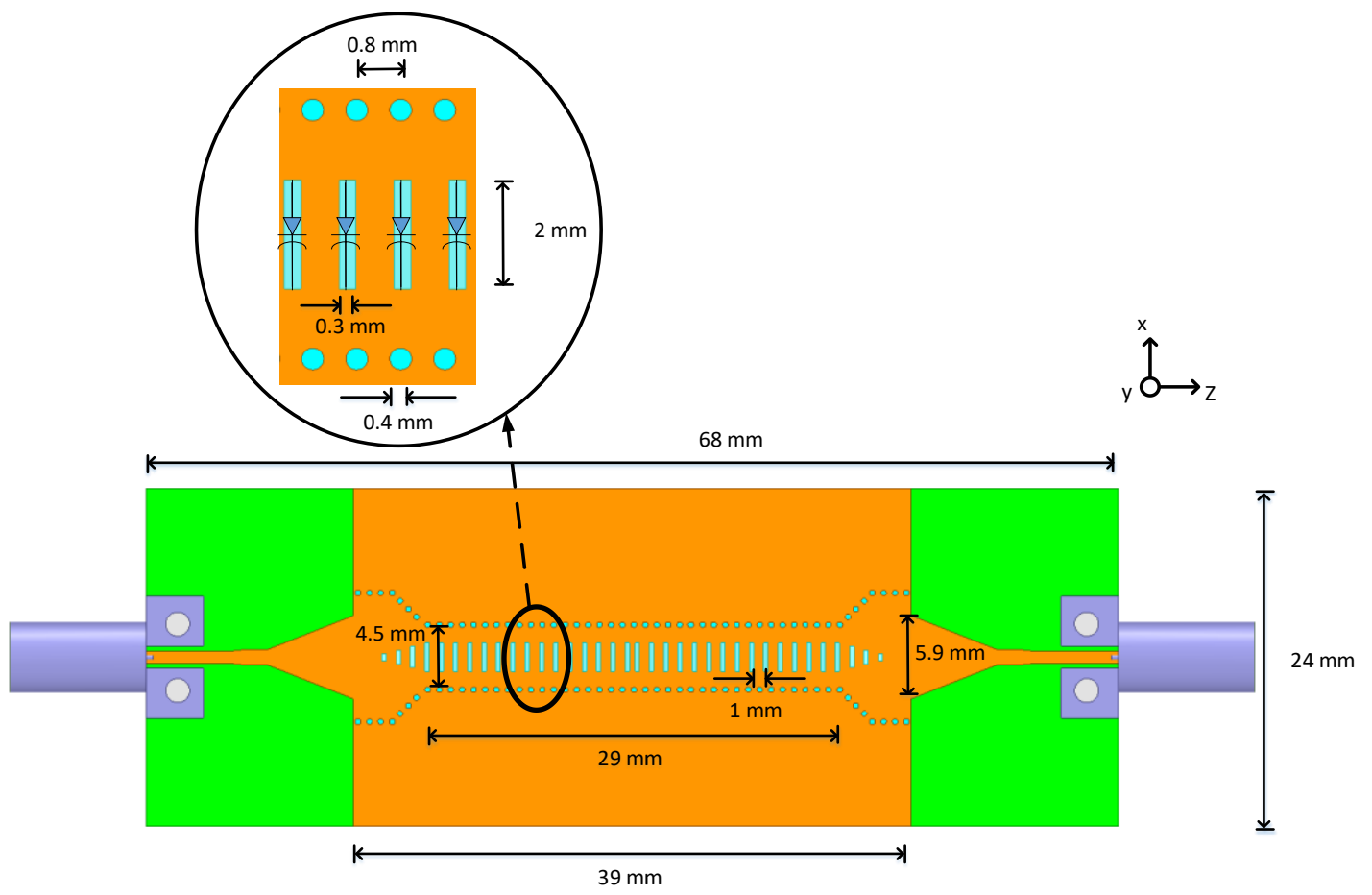

(b)

Figure 5.28. Schematic view of the reconfigurable SIW LWA with transverse slots [112]. (a) 3D view, (b) top view. 
The S-parameters of uniform switching topology for different capacitance values are presented in Fig. 5.29. Return loss is better than $10 \mathrm{~dB}$ in all cases over the frequency band of $28-29 \mathrm{GHz}$. The corresponding insertion loss ranges from 1.4 to $4 \mathrm{~dB}$ in all switching states.

The radiation patterns of the antenna, shown in Fig. 5.30, demonstrate an electronic beam-scanning range of $35^{\circ}$ with the peak realized gain in the range of $0.2-9.1 \mathrm{dBi}$. The reported patterns correspond to the realized gains of the antenna for different varactor capacitance values of $50 \mathrm{fF}, 0.5 \mathrm{pF}$, and $5 \mathrm{pF}$.

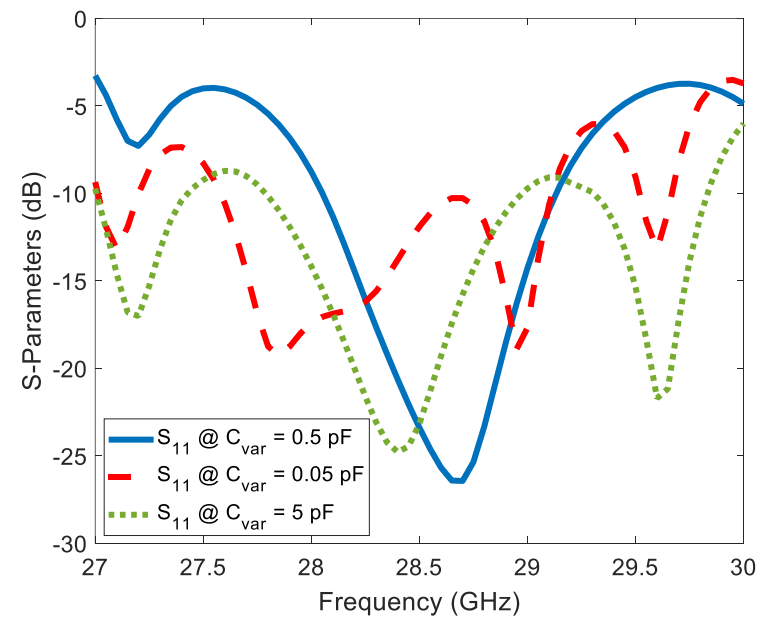

(a)

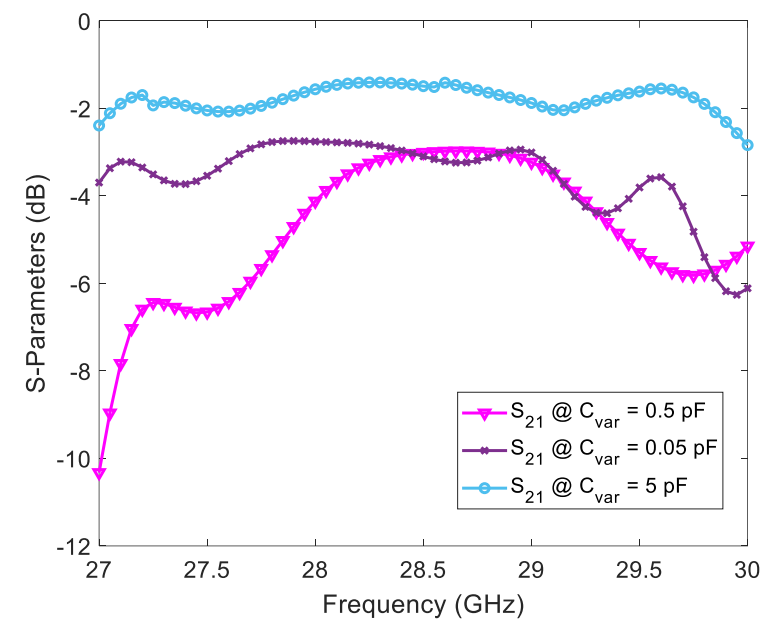

(b)

Figure 5.29. S-parameters of the proposed SIW LWA with uniform switching at $28.5 \mathrm{GHz}$ [112]. (a) $\mathbf{S}_{11}$, (b) $\mathbf{S}_{21}$. 


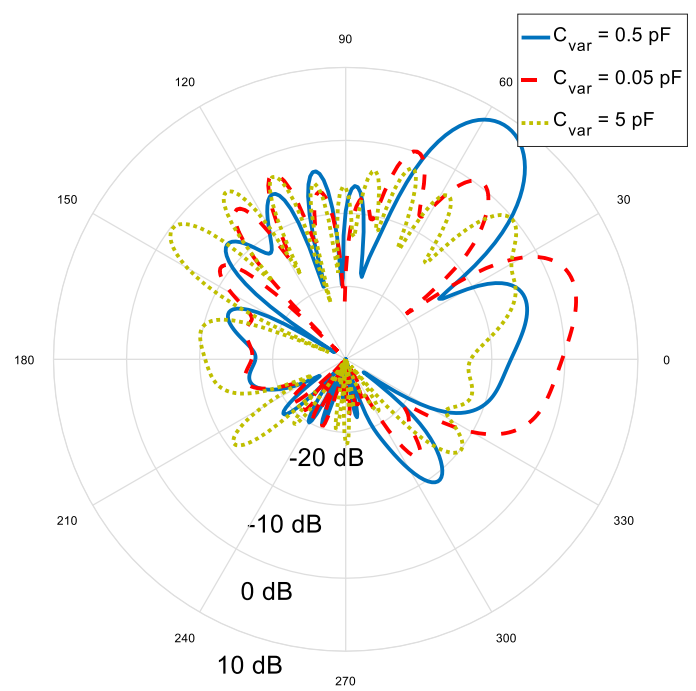

Figure 5.30. Radiation patterns of the proposed reconfigurable SIW LWA with uniform switching at E-plane (YZ-plane) [112].

Next, the non-uniform switching results were investigated [113]. This switching configuration provides more degrees of freedom in the antenna design and control over the radiation pattern. However, doing so increases the complexity and the cost of the antenna. To reduce the complexity of the design, only three different switching states were considered as follows; all "off," first half "on," and all "on." The S-parameters, presented in Fig. 5.31, show a return loss better than $10 \mathrm{~dB}$ from $28 \mathrm{GHz}$ to $29.5 \mathrm{GHz}$ in all states, while the insertion loss ranges from $1.5 \mathrm{~dB}$ to $7.8 \mathrm{~dB}$ over the frequency band of $28-29.5 \mathrm{GHz}$. The radiation patterns of the antenna at $28.5 \mathrm{GHz}$ are presented in Fig. 5.32, indicating that by sweeping the bias voltage, the antenna scans space from $60^{\circ}$ to $141^{\circ}$. The reported peak realized gain varies from $0.5 \mathrm{dBi}$ to $7.2 \mathrm{dBi}$, which agrees with the variations of the S-parameters at $28.5 \mathrm{GHz}$. As stated before, non-uniform biasing increases the number of possible configurations that leads to a more controllable radiation pattern at the expense of increasing the complexity and the cost. 


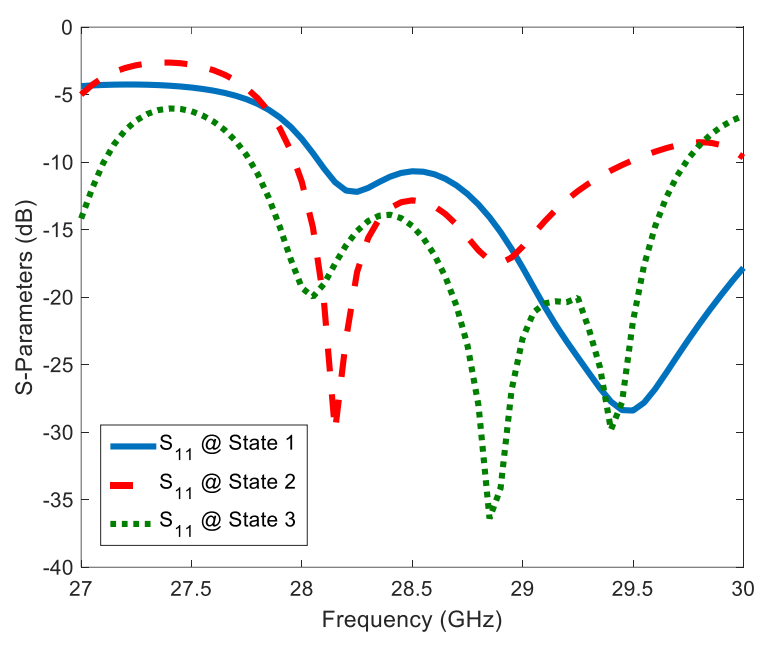

(a)

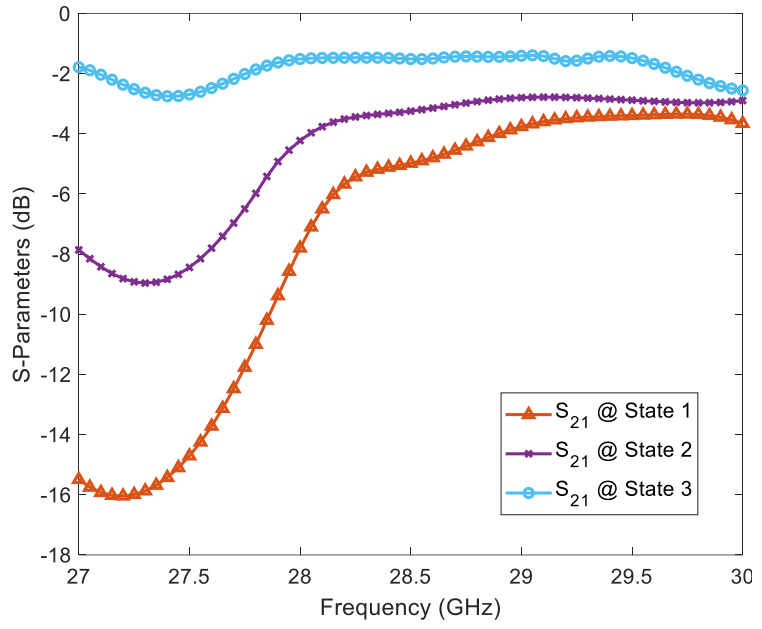

(b)

Figure 5.31. S-parameters of the proposed reconfigurable SIW LWA with non-uniform switching for different states (1) all off, (2) first half on, and (3) all on [113]. (a) $S_{11}$, (b) $S_{21}$.

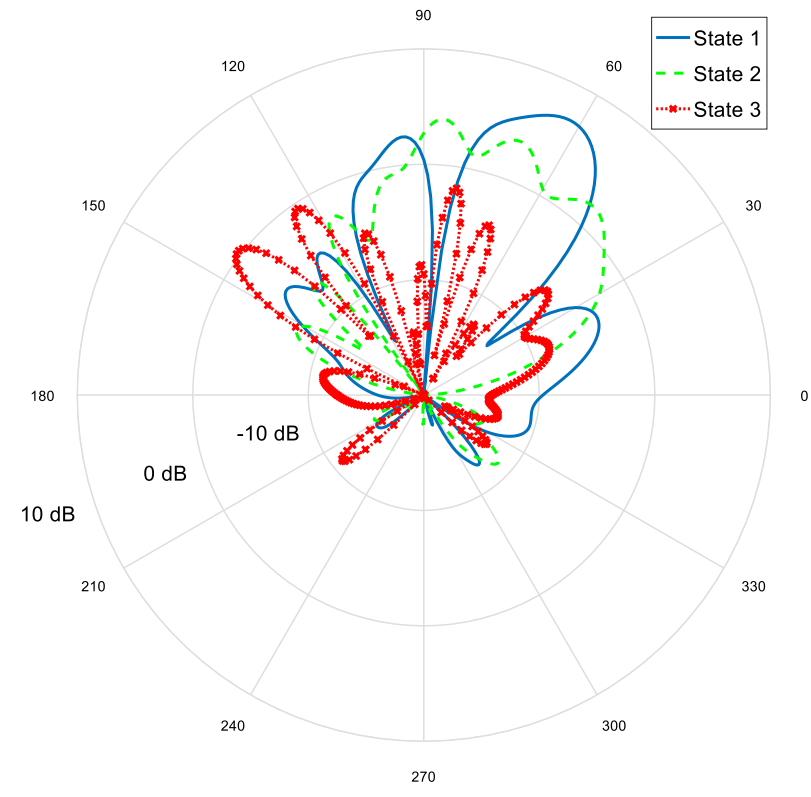

Figure 5.32. Radiation patterns of the proposed reconfigurable SIW LWA at 28.5 GHz and E-plane for different non-uniform switching states (1) all off, (2) first half on, and (3) all on [113].

\subsubsection{Reconfigurable SIW LWA with Offset Longitudinal Slots}

In the proposed design, the beam-steering is achieved by placing varactor diodes over the offset longitudinal slots, as presented in Fig. 5.33. Sweeping the reverse bias voltage causes the variation in series capacitance, series resonance frequency, and phase constant 
that consequently leads to beam-scanning. The length, width, and thickness of the antenna are $95 \mathrm{~mm}, 23 \mathrm{~mm}$, and $0.3 \mathrm{~mm}$, respectively. The proposed antenna consists of twenty-nine periodic cells. Each cell is a $1.6 \mathrm{~mm} \times 0.4 \mathrm{~mm}$ offset longitudinal rectangular slot. In the proposed design, switches are biased uniformly.

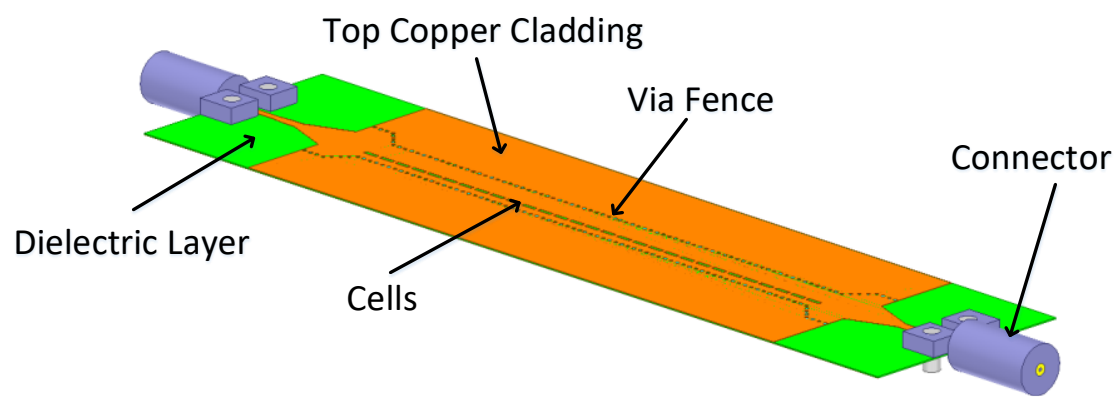

(a)

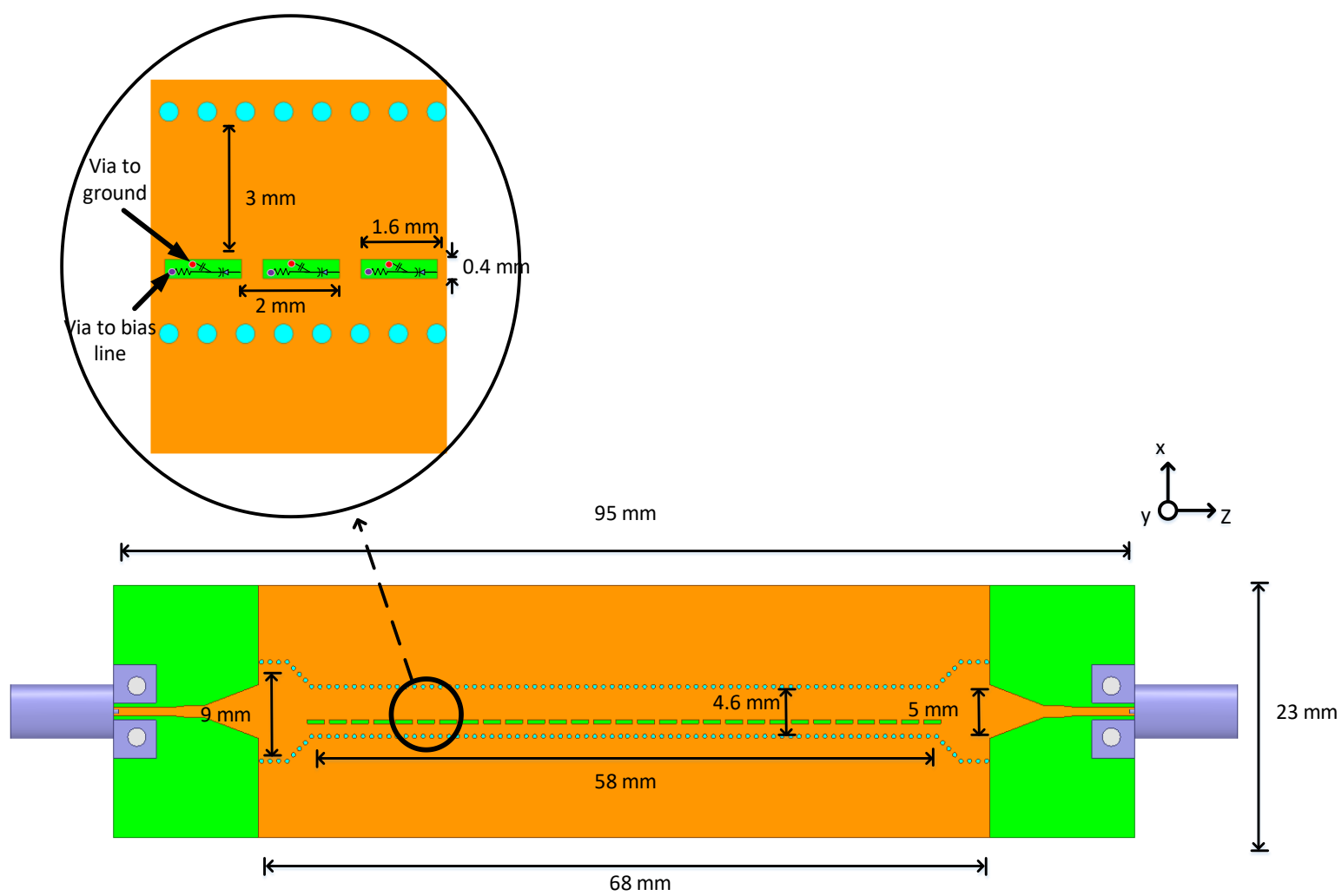

(b)

Figure 5.33. Schematic view of the proposed reconfigurable SIW LWA with longitudinal slots

[114]. (a) 3D view, (b) top view. 
The simulation results are obtained using ANSYS HFSS v19.2. The simulated Sparameters for different switch capacitance values are reported in Fig. 5.34. According to Fig. 5.34, the return loss is better than $10 \mathrm{~dB}$ in all states over the frequency bands of 27.6-28.2 GHz. The corresponding insertion loss ranges from $4 \mathrm{~dB}$ to $15 \mathrm{~dB}$ over the 10 dB impedance bandwidth (27.6-28.2 GHz).

The realized gain radiation patterns for different switch capacitance values are presented in Fig. 5.35, which indicates that the antenna scans a relatively wide space using a small variable capacitance range. Sweeping the varactor capacitance from $200 \mathrm{fF}$ to $250 \mathrm{fF}$ leads to a $27^{\circ}$ scanning range with low SLL and peak realized gain of $4.7 \pm 0.2$ $\mathrm{dBi}$ at $27.8 \mathrm{GHz}$. The low SLL, small gain variations, and wide beam-scanning rang of the proposed antenna make it an excellent candidate for $5 \mathrm{G}$ mm-wave applications.

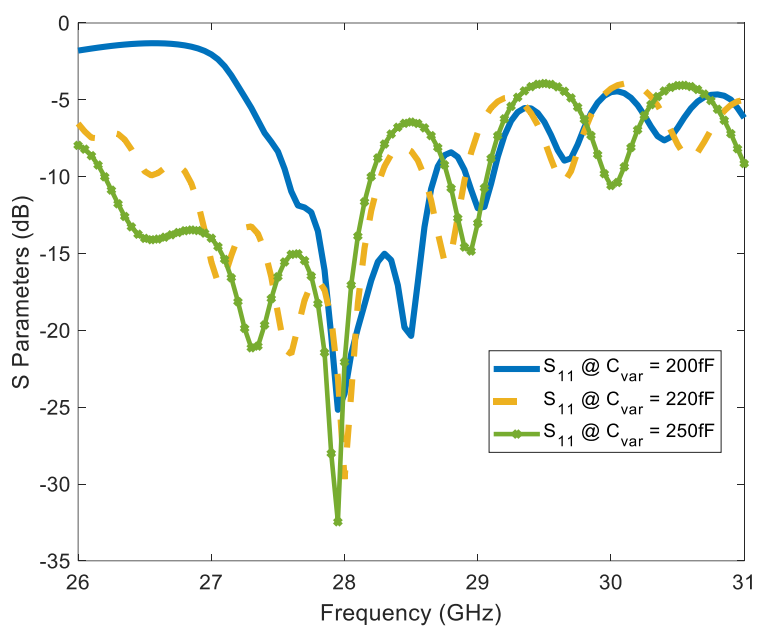

(a)

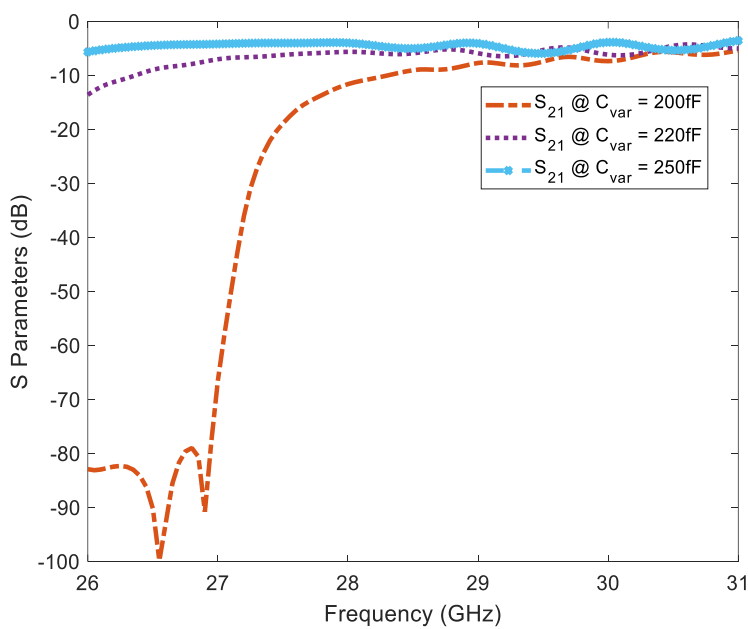

(b)

Figure 5.34. S-parameters of the proposed reconfigurable SIW LWA with offset longitudinal slots [114]. (a) $S_{11}$, (b) $S_{21}$. 


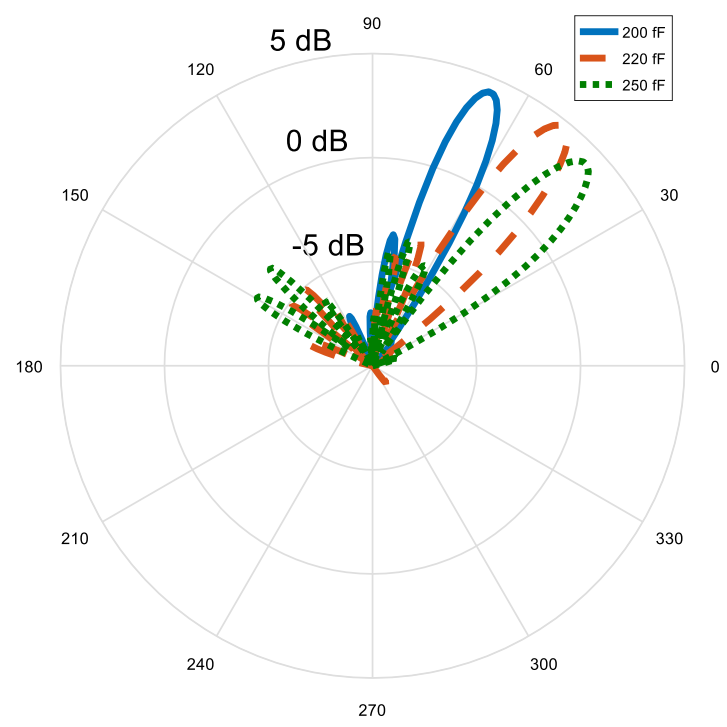

Figure 5.35. Radiation patterns of the proposed reconfigurable SIW LWA with longitudinal slots for different switch capacitance values at 27.8 GHz and E-plane (YZ-plane) [114].

\subsubsection{Reconfigurable SIW LWA Composed of Longitudinal Slots on the Backside}

In this section, an electronic beam-scanning SIW LWA is presented. Radiation occurs through narrow longitudinal slots offset from the central axis of the top copper cladding. The beam-scanning is achieved by mounting varactor diodes on the backside of the antenna. The key features of the proposed reconfigurable antenna are the low-profile and electronic beam-scanning capability using a single switch per cell located on the antenna's backside. Moreover, placing the components on the backside of the antenna reduces their unwanted effects on the radiation.

Variation in S-parameters of a unit cell leads to the modification of $\beta$ based on (5.1). According to (2.3), variation in $\beta$ changes $\theta_{0}$ confirming the electronic beam-steering capability. Assuming the propagation of the dominant mode ( $\left.\mathrm{TE}_{10}\right)$, the antenna's width is calculated by (2.6). Selecting $f_{c}=24 \mathrm{GHz}$ and $\varepsilon_{r}=2.2$ led to $w=4.6 \mathrm{~mm}$. The radiation length of the antenna is obtained from (2.4). $\Delta \theta$ and $\theta_{0}$ of the proposed structure at 28.5 $\mathrm{GHz}$ were chosen as $20^{\circ}$ and $30^{\circ}$, respectively. The extracted $L_{r}$ from (2.4) was $58 \mathrm{~mm}$. 
The antenna's total length depends on the feed length, determined by optimizing the return loss.

The antenna was fabricated on a multi-layer Rogers RT/Duroid 5880 substrate, as presented in Fig. 5.36. The antenna's length, width, and thickness are $95 \mathrm{~mm}, 24 \mathrm{~mm}$, and $0.32 \mathrm{~mm}$, respectively. The width of the antenna was chosen to achieve single-mode propagation, according to (2.5) and (2.6). To minimize the return loss, tapered via fences in addition to tapered microstrip transition were utilized at the input/output of the antenna. Each end of the structure was terminated by a k-connector, while one of them is terminated by a matched load to absorb the residual power since the maximum radiation efficiency of an LWA is 90\%. The geometrical parameters are listed in Table 5.4.

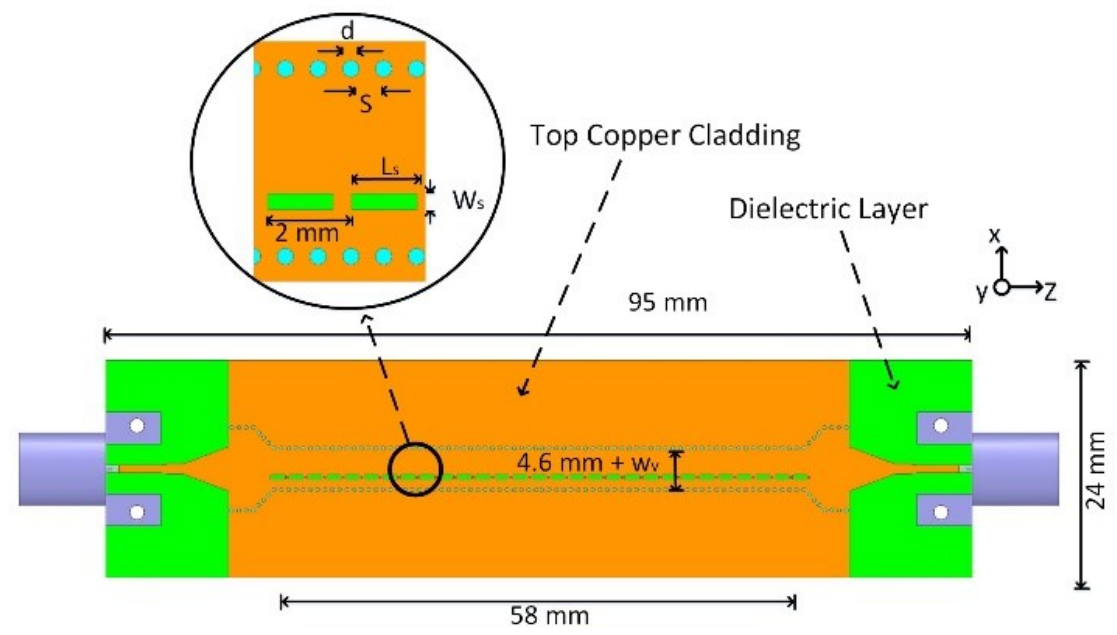

(a)

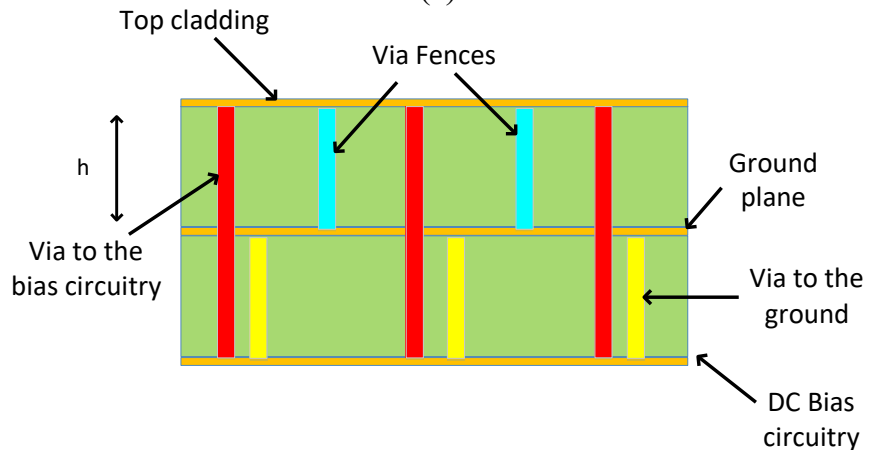

(b)

Figure 5.36. Schematic view of the proposed reconfigurable SIW-based LWA [115]. (a) Top view, (b)

Side view. 


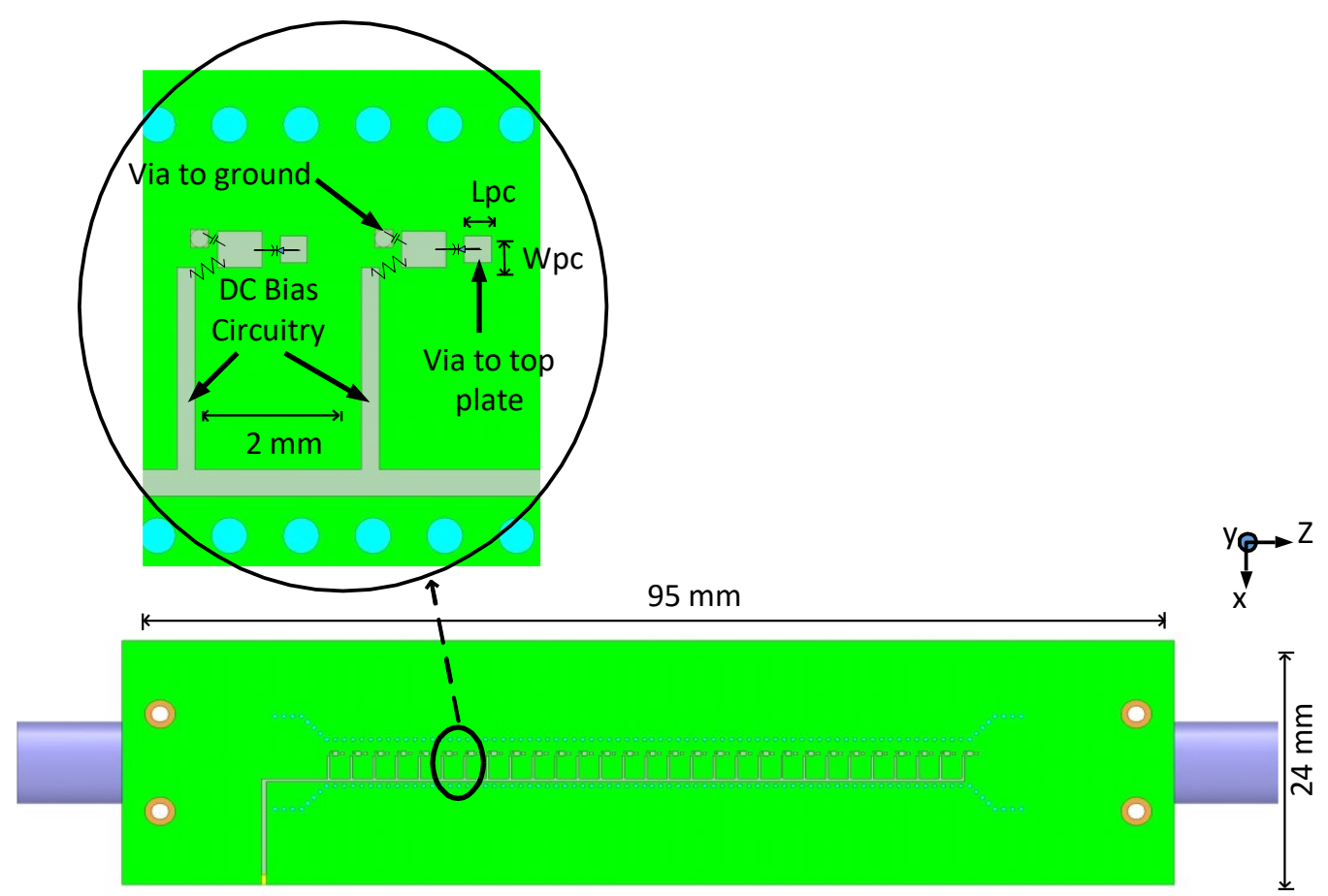

(c)

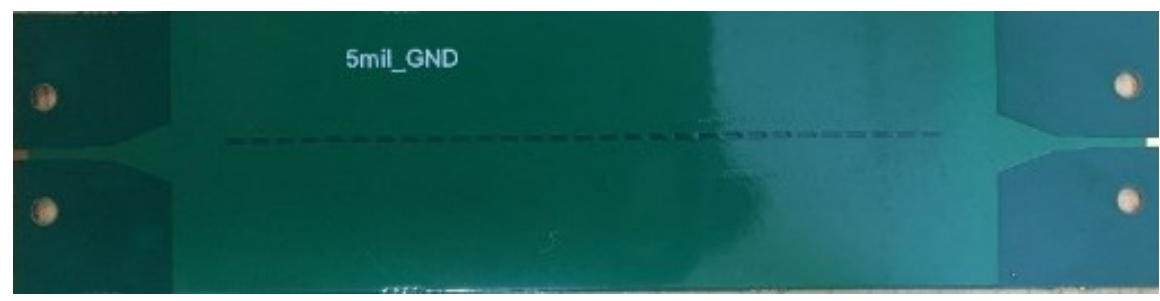

(d)

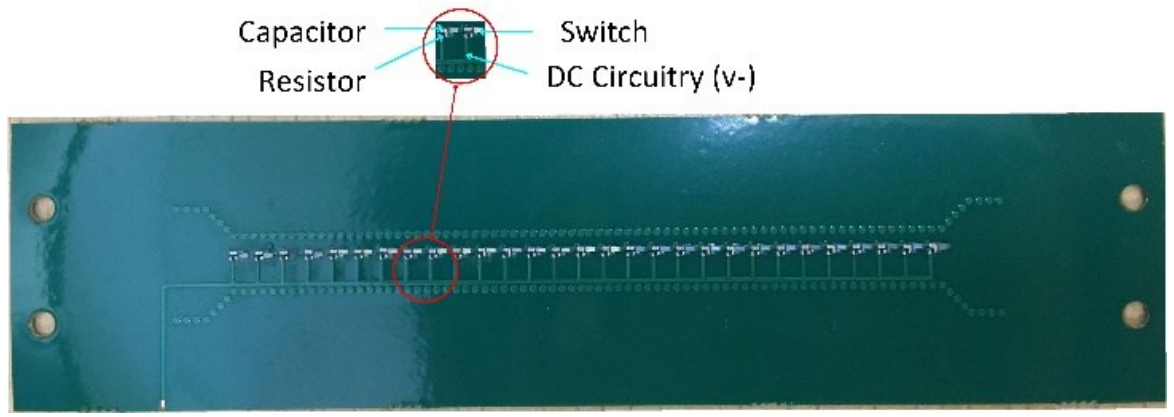

(e)

Figure 5.36. Schematic view of the proposed reconfigurable SIW-based LWA (cont'd) [115]. (c) Bottom view, (d) Top view of the fabricated LWA, and (e) Bottom view of the fabricated antenna.

Table 5.4. Important geometrical parameters of the proposed LWA [115].

\begin{tabular}{|l|l|l|l|l|l|l|l|l|}
\hline Parameter & $h$ & $s$ & $d$ & $W_{v}$ & $L_{s}$ & $W_{s}$ & $L_{p c}$ & $W_{p c}$ \\
\hline Value (mm) & 0.127 & 0.8 & 0.4 & 0 & 1.6 & 0.5 & 0.3 & 0.3 \\
\hline
\end{tabular}


The proposed antenna contains twenty-nine reconfigurable cells with $2 \mathrm{~mm}$ spacing. The period of slots was chosen to allow the propagation of only the fundamental space harmonic. Each cell includes a $L_{s} \times W_{s}$ longitudinal rectangular slot offset from the central axis. The fabrication design rules limit the cell size as well.

The proposed reconfigurable cell contains a MAVR-000120-14110P GaAs varactor diode as a switch, a chip capacitor GRM-0335C1H4R7CA01D as a DC block, and a chip resistor ERA-1AEB102C as an RF block. Similar to the Section 5.3.3, the varactor diode's anode was grounded through the side via fence while the cathode was connected to the bias circuitry through the RF block. The bias circuitry was located under the ground plane to keep the structure compact, as shown in Fig. 5.36(b). However, the components were assembled on the backside of the antenna to minimize their footprints' undesired effect on the radiation pattern. This also eased the fabrication and assembly procedure since more space is available on the backside of the antenna. The varactor diodes, DC blocks, and RF blocks are assembled on the pads using BGA technique to minimize the increase of inductance and resistance due to the assembly. The DC pad of the bias line is connected to the DC source through a thin and durable wire.

The full-wave simulation results were obtained using ANSYS HFSS v19.2. As stated before, the varactor diode acts as a variable capacitor. Therefore, sweeping the switch's bias voltage changes the resonance frequency and the impedance of the cell. It also leads to the variations of $\beta$ and $\theta_{0}$, according to (5.1) and (2.3). To investigate the electronic beam-steering capability of the proposed design, four switching states are investigated as follows; (1) all switches are biased at $1 \mathrm{~V}$, (2) all switches are biased at $4 \mathrm{~V}$, (3) all switches are biased at $11 \mathrm{~V}$, and (4) all switches are biased at $15 \mathrm{~V}$. According to the 
varactor diode datasheet [147], increasing the voltage from $1 \mathrm{~V}$ to $15 \mathrm{~V}$ reduces its capacitance from $0.9 \mathrm{pF}$ to $0.15 \mathrm{pF}$. Moreover, the $\mathrm{Q}$ factor of the varactor diode at $4 \mathrm{v}$ and $50 \mathrm{MHz}$ is 3000 [147]. Similar to the previous measurement procedure, S-parameters were measured using a Rohde \& Schwarz ZVA-67 VNA, and the radiation pattern was measured at the anechoic chamber using an MI-12A-26 standard gain horn antenna, as shown in Fig. 5.37.

Simulated and measured S-parameters for four different switching states are reported in Fig. 5.38. According to Fig. 5.38, a return loss better than $10 \mathrm{~dB}$ over the frequency band of $27-30 \mathrm{GHz}$ was achieved. Since the implemented varactor diodes have constant return loss, there is small variation in the return loss by sweeping the reverse bias voltage. The corresponding insertion loss varies from $13 \mathrm{~dB}$ to $20 \mathrm{~dB}$. The discrepancies between measured and simulated results are discussed through sensitivity analysis.

Normalized radiation patterns for different switching states at $27 \mathrm{GHz}, 28.5 \mathrm{GHz}$, and $30 \mathrm{GHz}$ are presented in Fig. 5.39, confirming the electronic beam-scanning. Only the Eplane (YZ-plane) radiation pattern is reported since tilting the antenna under test was beyond the capability of the measurement setup. According to Figs. 5.39(c)-(d), sweeping the reverse bias voltage from $1 \mathrm{~V}$ to $15 \mathrm{~V}$ results in a $25^{\circ}$ scanning range at $28.5 \mathrm{GHz}$.

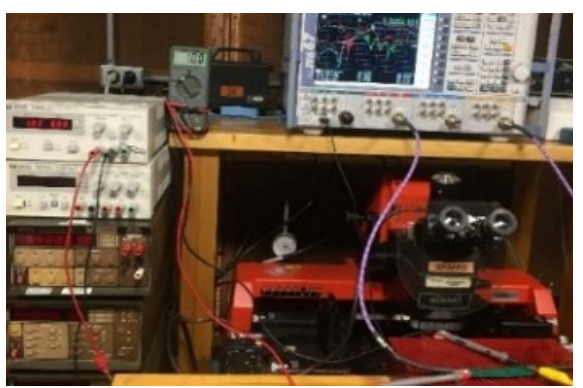

(a)

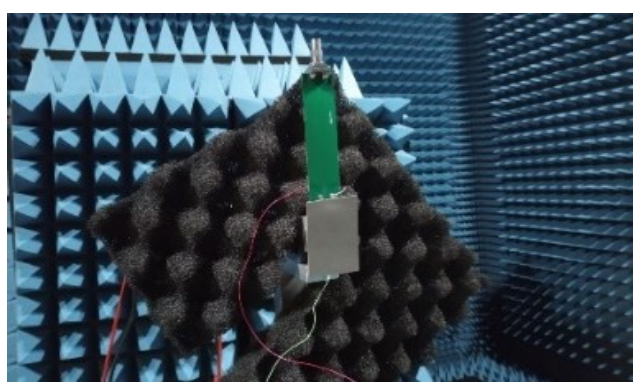

(b)

Figure 5.37. Antenna under test in (a) S-parameters measurement, and (b) anechoic chamber [115]. 


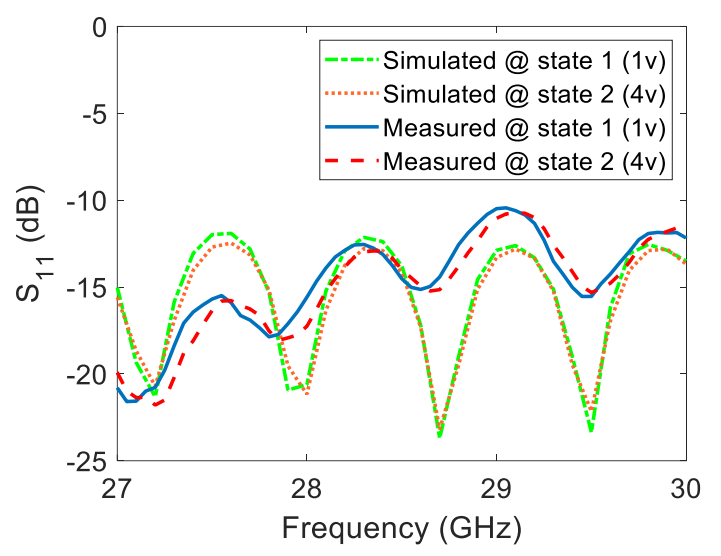

(a)

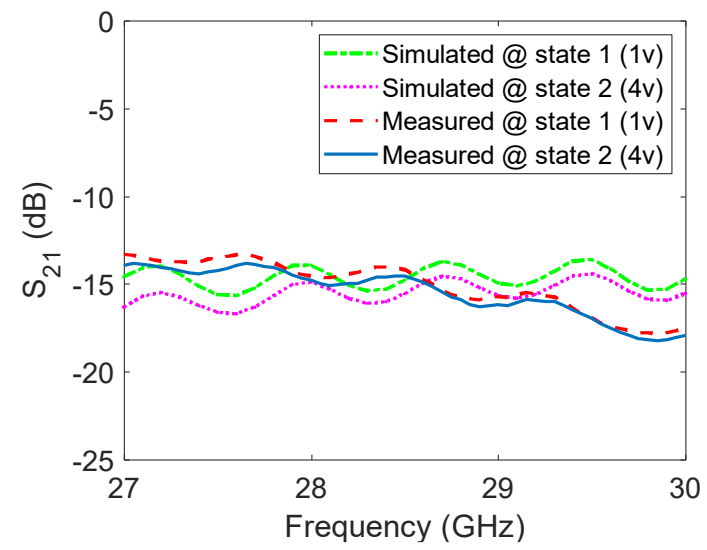

(c)

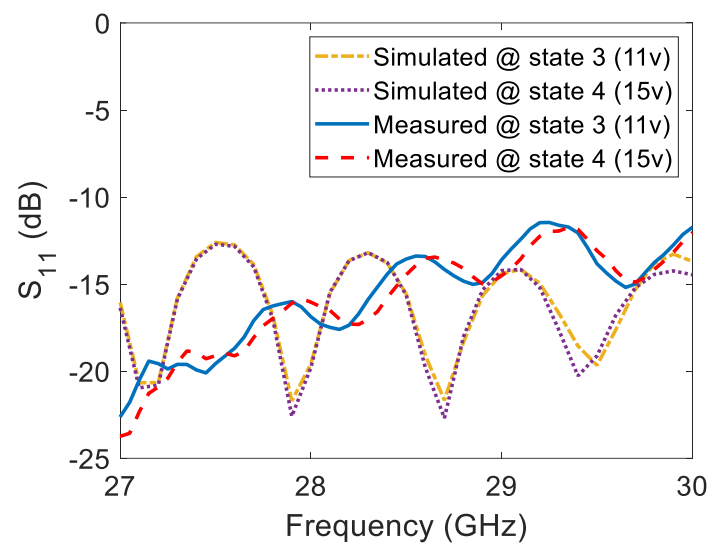

(b)

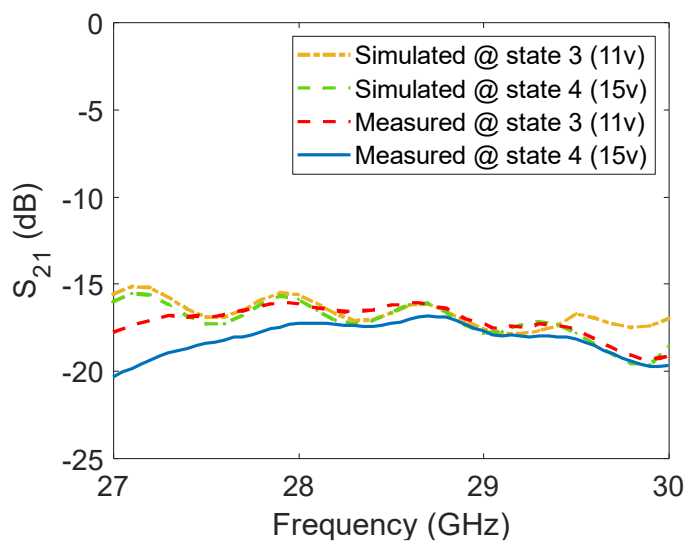

(d)

Figure 5.38. S-parameters of the proposed reconfigurable SIW LWA [115]. (a) S11 at states 1 and 2 ,

(b) $S_{21}$ at states 3 and 4, (c) $S_{11}$ at states 1 and 2, (d) $S_{21}$ at states 3 and 4.

As stated before, the main-lobe pointing angle changes by sweeping the frequency due to the leaky-wave nature of the antenna [28]. According to Fig. 5.39, the measured and the simulated frequency beam-squint in the frequency band of $27-30 \mathrm{GHz}$ are $5^{\circ}$ and $3^{\circ}$, respectively. Realization of the proposed design on Rogers RT/Duroid 5880 substrate led to reducing the frequency beam-squint. The measured and simulated peak realized gains are demonstrated in Fig. 5.40, indicating the proposed LWA radiates with $9 \pm 0.8 \mathrm{dBi}$ gain at $28.5 \mathrm{GHz}$ for different switch states. 


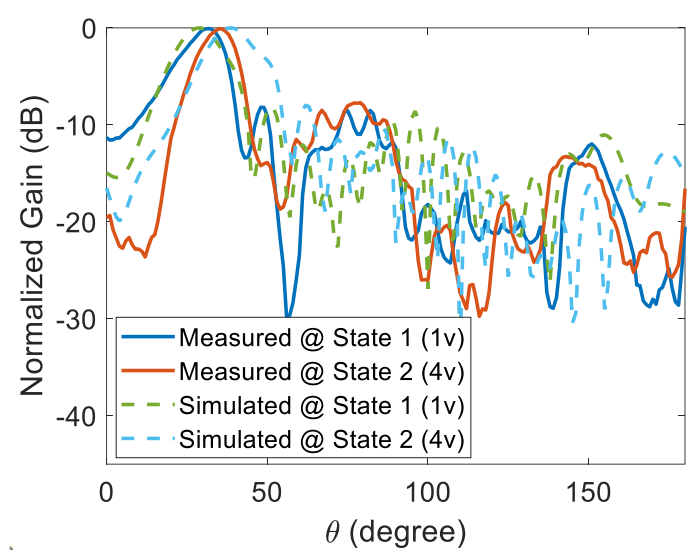

(a)

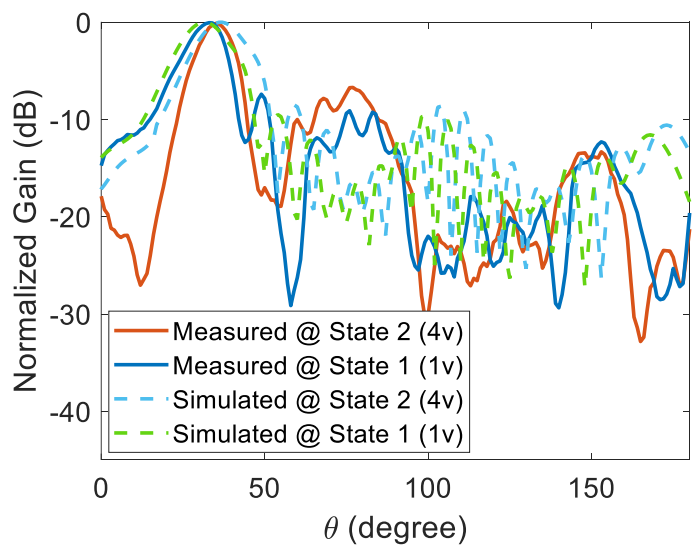

(c)

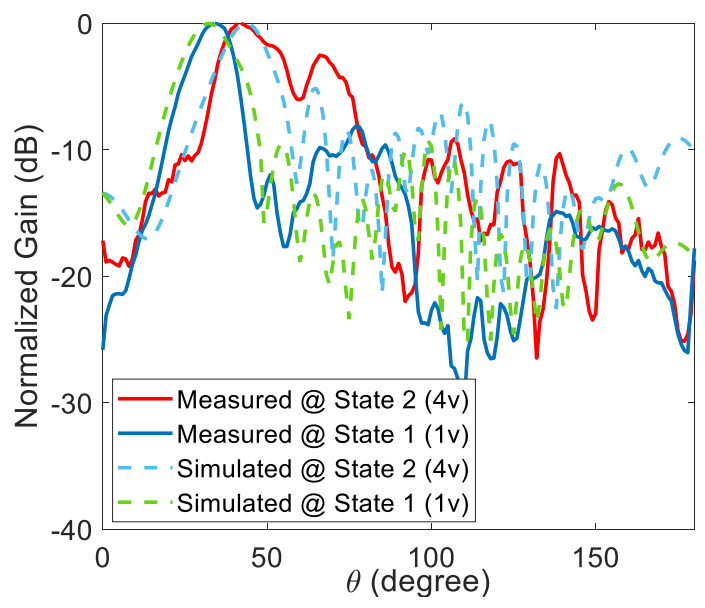

(e)

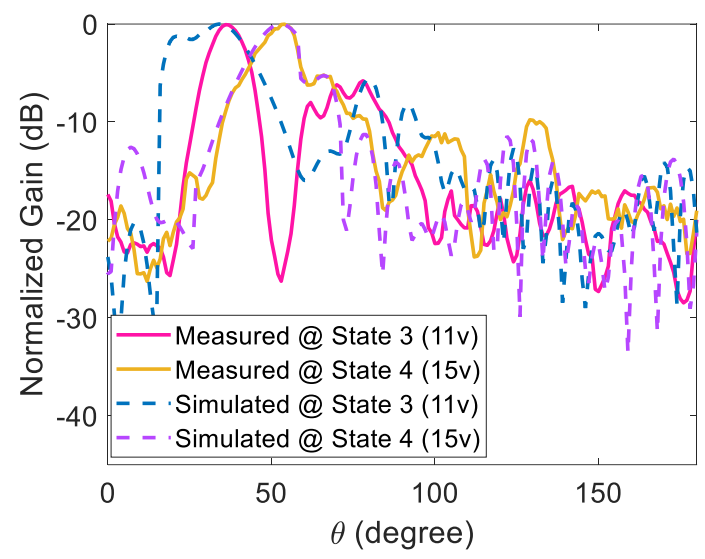

(b)

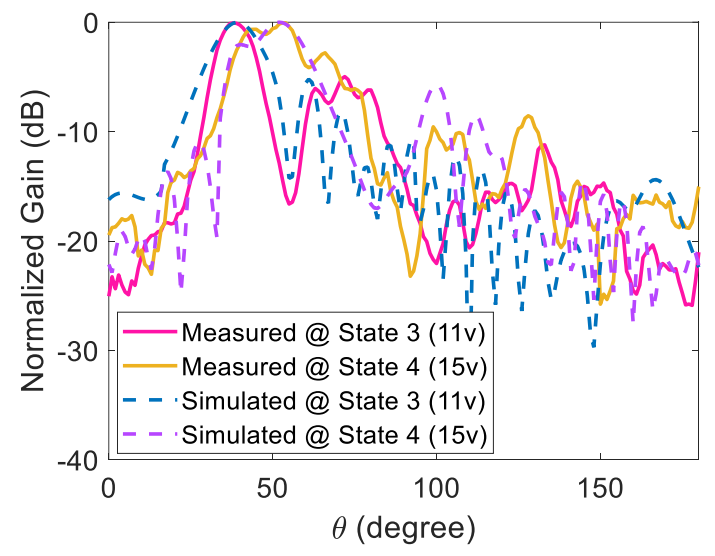

(d)

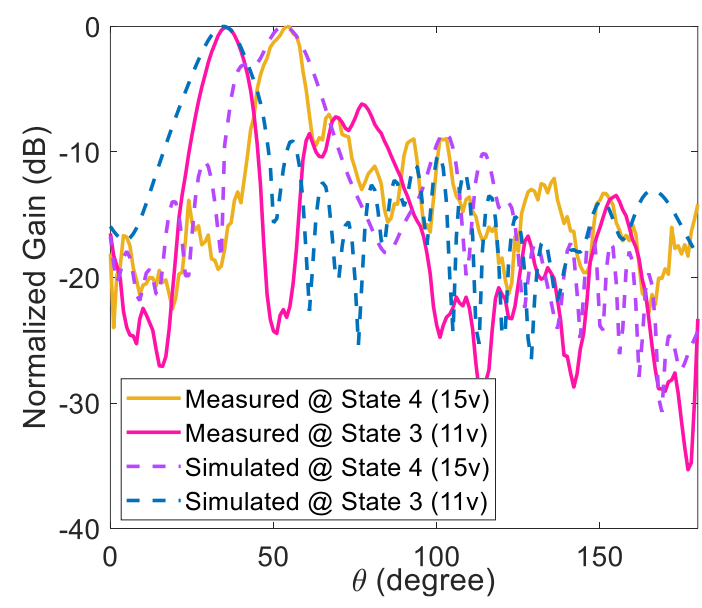

(f)

Figure 5.39. Normalized radiation patterns of the proposed electronic beam-steering antenna for different states in E-plane (YZ plane) [115]. (a) States 1 and 2 at 27GHz, (b) States 3 and 4 at 27

GHz, (c) States 1 and 2 at $28.5 \mathrm{GHz}$, (d) States 3 and 4 at $28.5 \mathrm{GHz}$, (e) States 1 and 2 at $30 \mathrm{GHz}$, (f) States 3 and 4 at $30 \mathrm{GHz}$. 


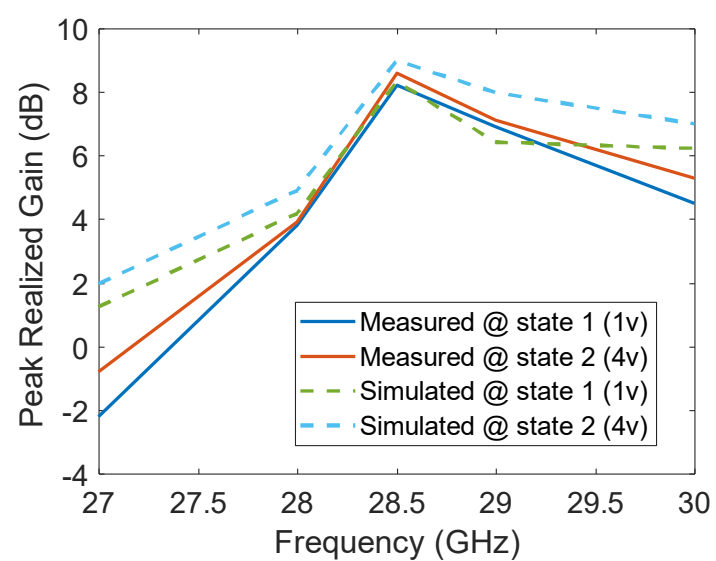

(a)

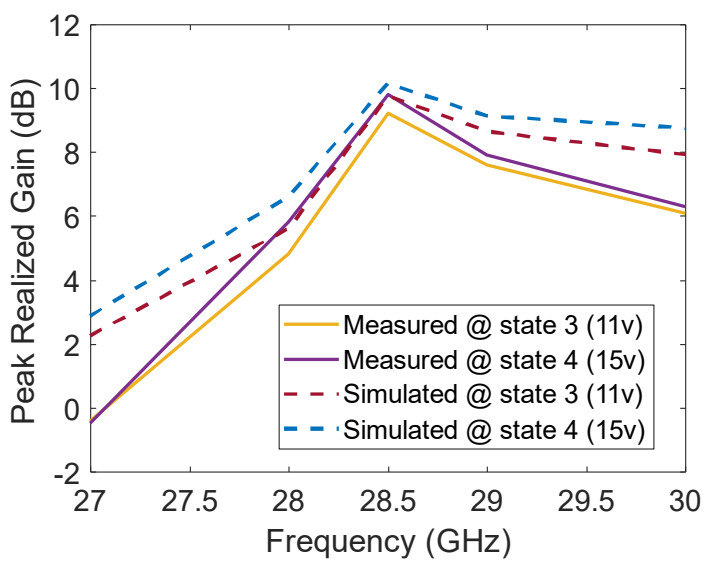

(b)

Figure 5.40. Peak realized gain of the proposed reconfigurable antenna [115]. (a) States 1 and 2, (b)

States 3 and 4.

The antenna was fabricated on a thin substrate to achieve a low-profile structure. This resulted in high conductor loss and lower gain. Increasing the number of reconfigurable cells without changing the period results in a higher directivity at the expense of increasing the cost, size, and the loss of the antenna. The SLL can also be reduced by tapering the slots [54], the via fence [52], the period of cells [53], or the feed transition [61]. In the proposed design, all varactor diodes had similar applied bias voltage. Biasing them individually may result in a broader beam-scanning range since more switching states are achievable. However, this is achieved at the expense of increasing the complexity and the cost of the device. The assembly process can introduce additional reactive load to each cell resulting in variations in the input impedance and $\beta$. If air gaps exist inside the via connecting the top plane to the varactor diode, the inductance of each cell changes as well. These can cause the discrepancies between measured and simulated S-parameters, radiation patterns, and peak realized gain.

A parametric study was carried out to investigate the discrepancies between measured and simulated results more carefully. The effects of the variations of parameters such as 
the thickness of the layer $(h)$, extended width of the waveguide section $\left(W_{v}\right)$, via spacing $(s)$, and via diameter $(d)$ on S-parameters and radiation patterns are reported in Figs. 5.41 and 5.42. According to Figs. 5.41 and 5.42, $h, s$, and $W_{v}$ contribute the most to the discrepancies between measurements and simulations.

As stated before, bending of the thin substrate under physical pressure results in discrepancies as well. The antenna was bent as indicated in Fig. 5.43 to investigate such a possibility. To simulate this case, the vertical distance from the horizon was gradually changed to $h_{b}=11 \mathrm{~mm}$ by moving from the center towards the two connectors. Sparameters, normalized radiation patterns, and peak realized gain of the bent reconfigurable SIW LWA are reported in Figs. 5.44-5.46, which confirm the degradations in antenna performance in terms of beam-scanning, SLL, gain, and Sparameters.

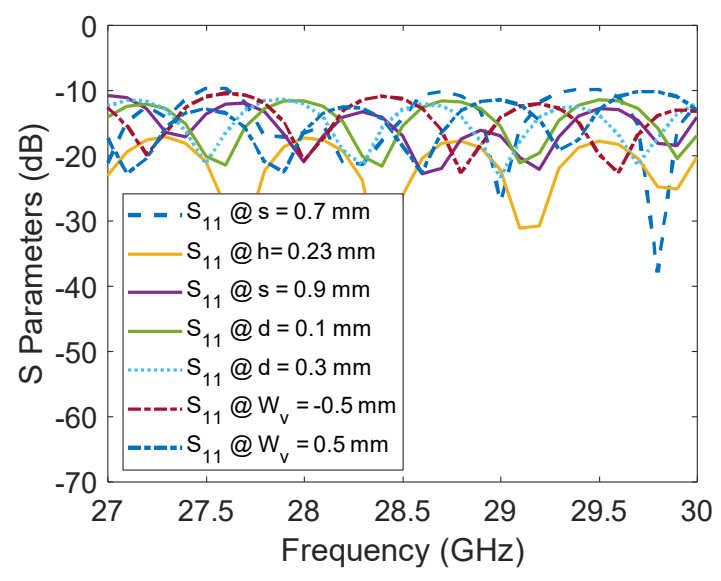

(a)

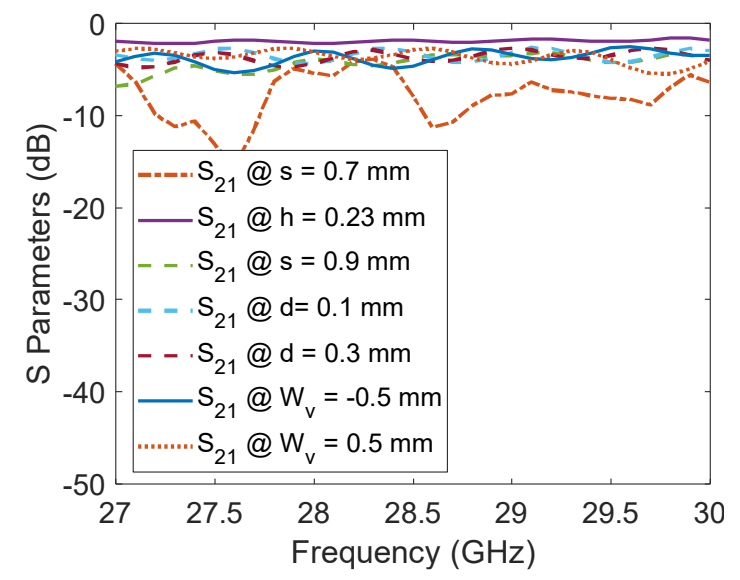

(b)

Figure 5.41. S-parameters for different geometrical parameters at state 2 [115]. (a) $S_{11}$ for different values of $h, s, d$, and $W_{v}$, (b) $S_{21}$ for different values of $h, s, d$, and $W_{v}$. 


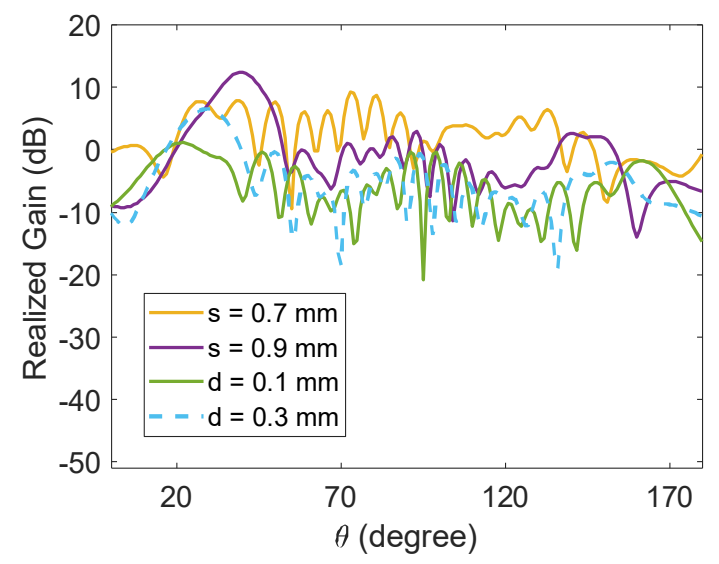

(a)

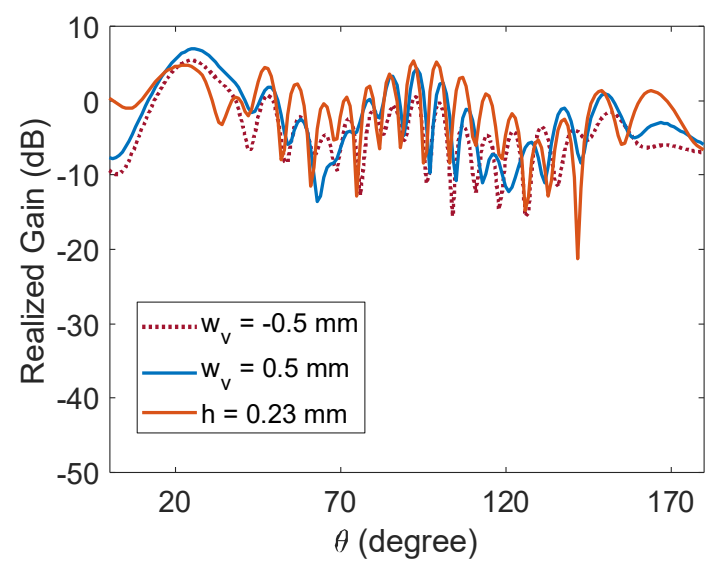

(b)

Figure 5.42. Radiation patterns for different geometrical configurations at state 2 and $28.5 \mathrm{GHz}$

[115]. (a) Different values of $s$ and $d$, (b) Different values of $h$ and $W_{v}$.

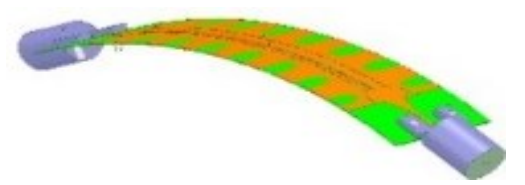

(a)

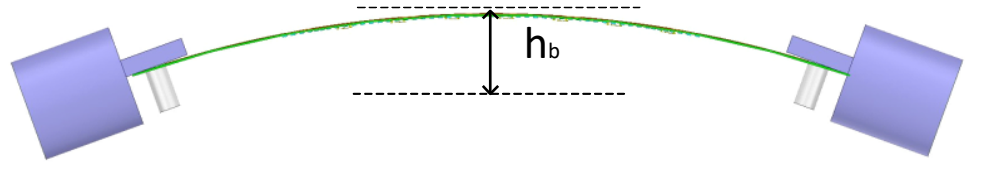

(b)

Figure 5.43. Bent reconfigurable antenna [115]. (a) 3D view, (b) Side view.

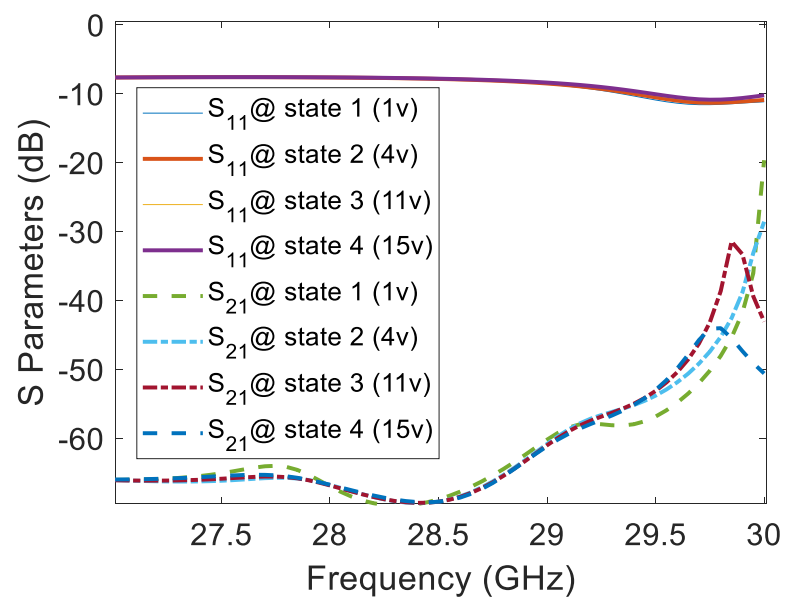

Figure 5.44. S-parameters of the bent reconfigurable antenna [115]. 


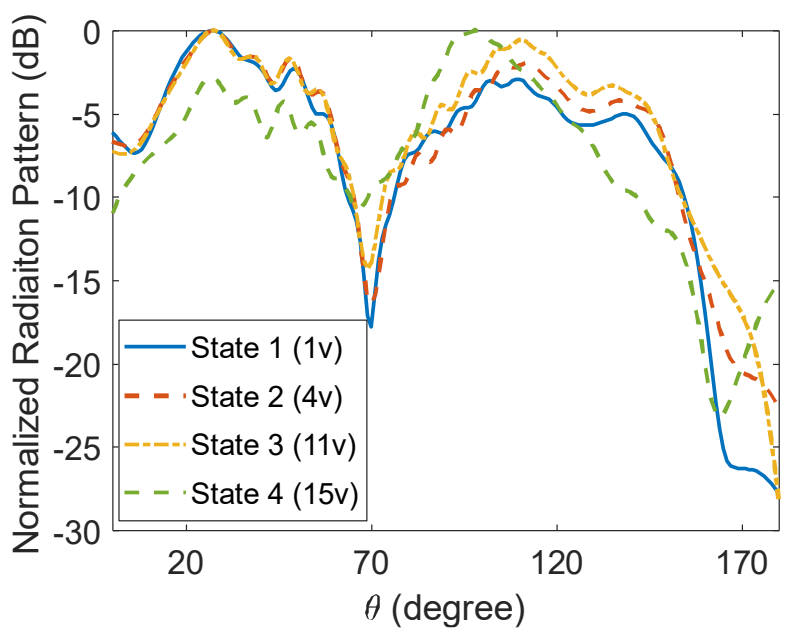

Figure 5.45. Normalized radiation pattern of bent reconfigurable SIW LWA [115].

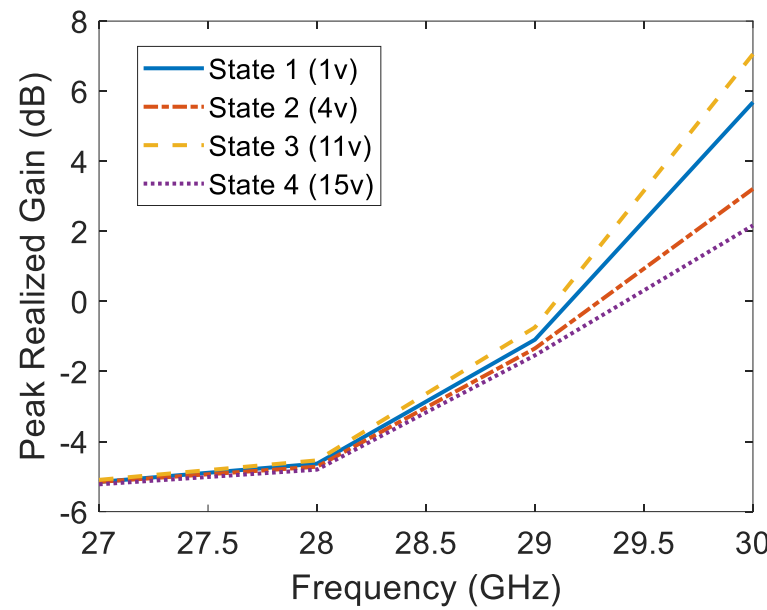

Figure 5.46. Peak realized gain of the bent electronic beam-scanning SIW LWA [115].

A comparison has been made in Table 5.5 with state-of-the-art electronic beamscanning antennas reported in [108]-[111] and [128]. The beam-scanning range of the reconfigurable SIW LWA proposed here is higher than [108], [109], and [128]. The wider beam-scanning ranges of [110] and [111] are because of the different approaches that were implemented. The reported structure in [110] is a corrugated SIW reconfigurable antenna, whereas electronic beam-scanning in [111] was achieved by implementing sets of the variable via posts in a cavity-based SIW antenna. The proposed antenna was fabricated on a thin substrate to achieve a low-profile structure at the expense of higher conductor loss as compared to [109]. This is the main reason for the 
smaller gain compared to [109], a corrugated SIW-based antenna. Our target frequency band is mm-wave, which is different from the reported frequency bands in [108]-[111] and [128]. The performance of the antennas reported in [108]-[111] and [128] degrade if they are scaled up to mm-wave frequency band.

In the proposed design, sweeping the reverse bias voltage in the range of $1-15 \mathrm{~V}$ led to a $25^{\circ}$ beam-scanning range at $28.5 \mathrm{GHz}$ with the peak realized gain of $9 \pm 0.8 \mathrm{dBi}$ [115]. Low-profile, medium gain, small gain variation, simplicity of the design, and electronic beam- steering capability make the proposed LWA a suitable candidate for $5 \mathrm{G}$ wireless networks and V2X communications.

\subsubsection{Miniaturized SIW LWA with Offset Longitudinal Slot}

Miniaturizing the antennas is beneficial in wireless devices. The proposed antenna is illustrated in Fig. 5.47. To miniaturize the reconfigurable SIW LWA, bias circuitry is integrated into the RF ground. The total length, width, and thickness of the antenna are $148 \mathrm{~mm}, 24 \mathrm{~mm}, 0.127 \mathrm{~mm}$, respectively. Each reconfigurable cell is a longitudinal rectangular slot containing a varactor diode, an RF block, and a DC block. Due to the small size of the slots at the top plane, the components were located on the ground plane.

Table 5.5. Comparison Among the Similar Reported Electronic Beam-Steering Antennas [115].

\begin{tabular}{|c|c|c|c|c|}
\hline Design & $\begin{array}{c}\text { Scanning } \\
\text { Range }\end{array}$ & Average Gain & $\begin{array}{c}\text { Center } \\
\text { Frequency }\end{array}$ & $\begin{array}{c}\text { Radiating } \\
\text { Length }\end{array}$ \\
\hline $\begin{array}{c}\text { Our proposed } \\
\text { reconfigurable } \\
\text { SIW LWA }\end{array}$ & $25^{\circ}$ & $9 \pm 0.8 \mathrm{dBi}$ & $28.5 \mathrm{GHz}$ & $5.5 \times \lambda$ \\
\hline$[128]$ & $17.5^{\circ}$ & $-5.8 \pm 0.25 \mathrm{dBi}$ & $3.23 \mathrm{GHZ}$ & $0.75 \times \lambda$ \\
\hline$[108]$ & $22^{\circ}$ & $5.53 \pm 1.5 \mathrm{dBi}$ & $5.2 \mathrm{GHZ}$ & $6 \times \lambda$ \\
\hline$[109]$ & $25^{\circ}$ & $11.3 \pm 1.15 \mathrm{dBi}$ & $5.8 \mathrm{GHZ}$ & $3.8 \times \lambda$ \\
\hline$[110]$ & $72^{\circ}$ & $5.6 \pm 0.3 \mathrm{dBi}$ & $4.5 \mathrm{GHZ}$ & $3.6 \times \lambda$ \\
\hline$[111]$ & $71^{\circ}$ & $7.8 \pm 1.4 \mathrm{dBi}$ & $2.6 \mathrm{GHZ}$ & $1.5 \times \lambda$ \\
\hline
\end{tabular}




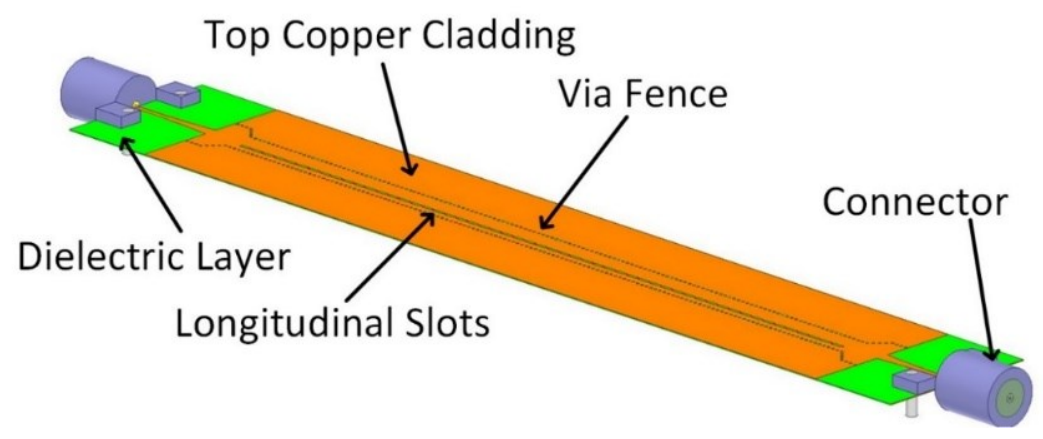

(a)

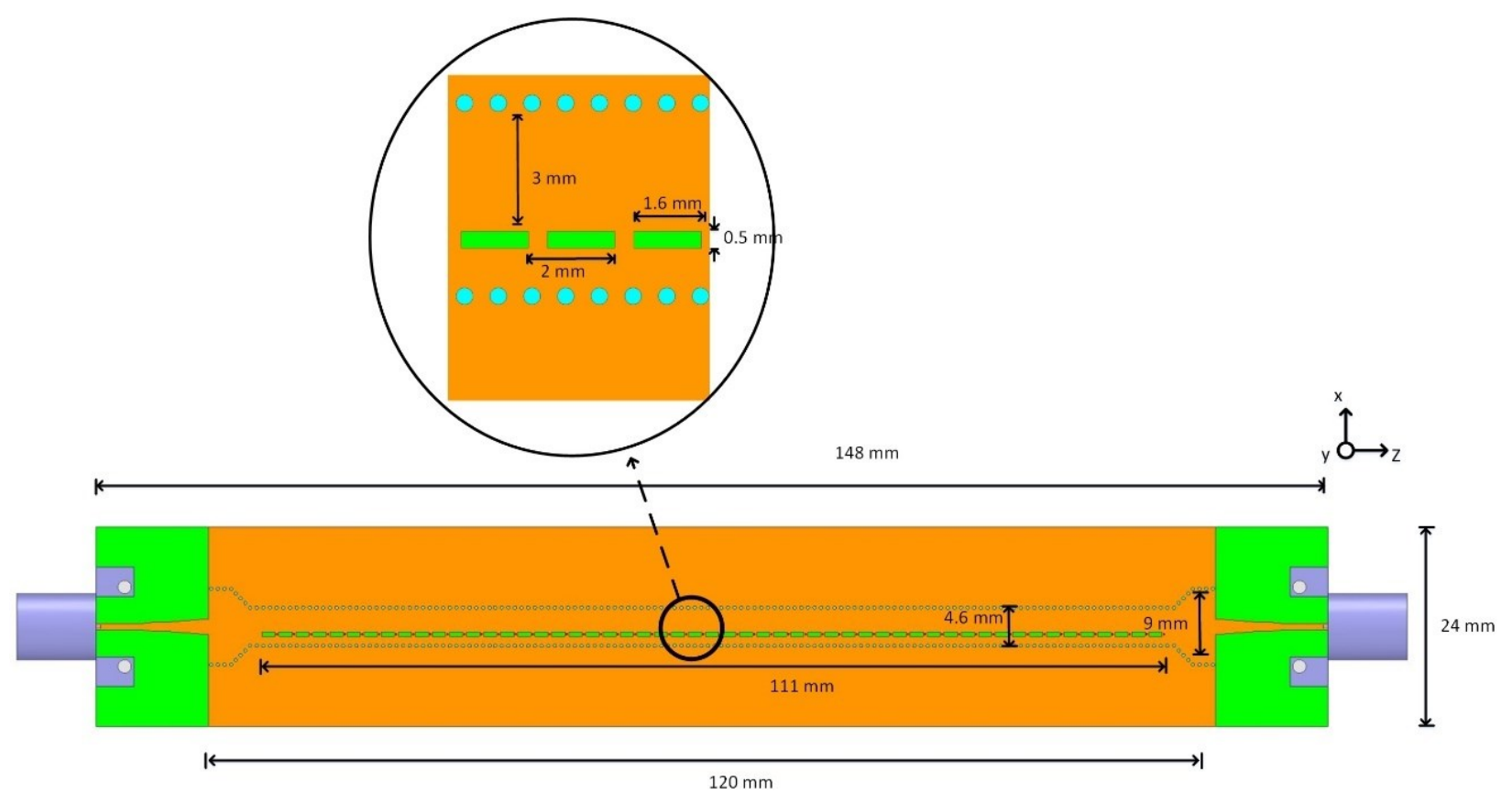

(b)

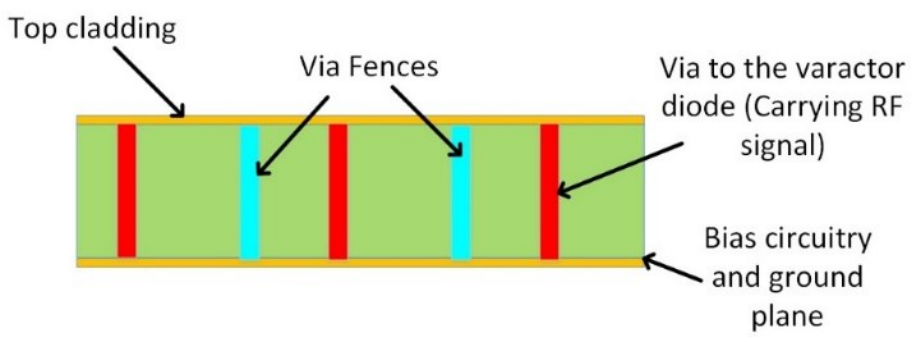

(c)

Figure 5.47. Schematic view of the proposed miniaturized reconfigurable antenna. (a) 3D view,

(b) Top view, (c) Side view. 


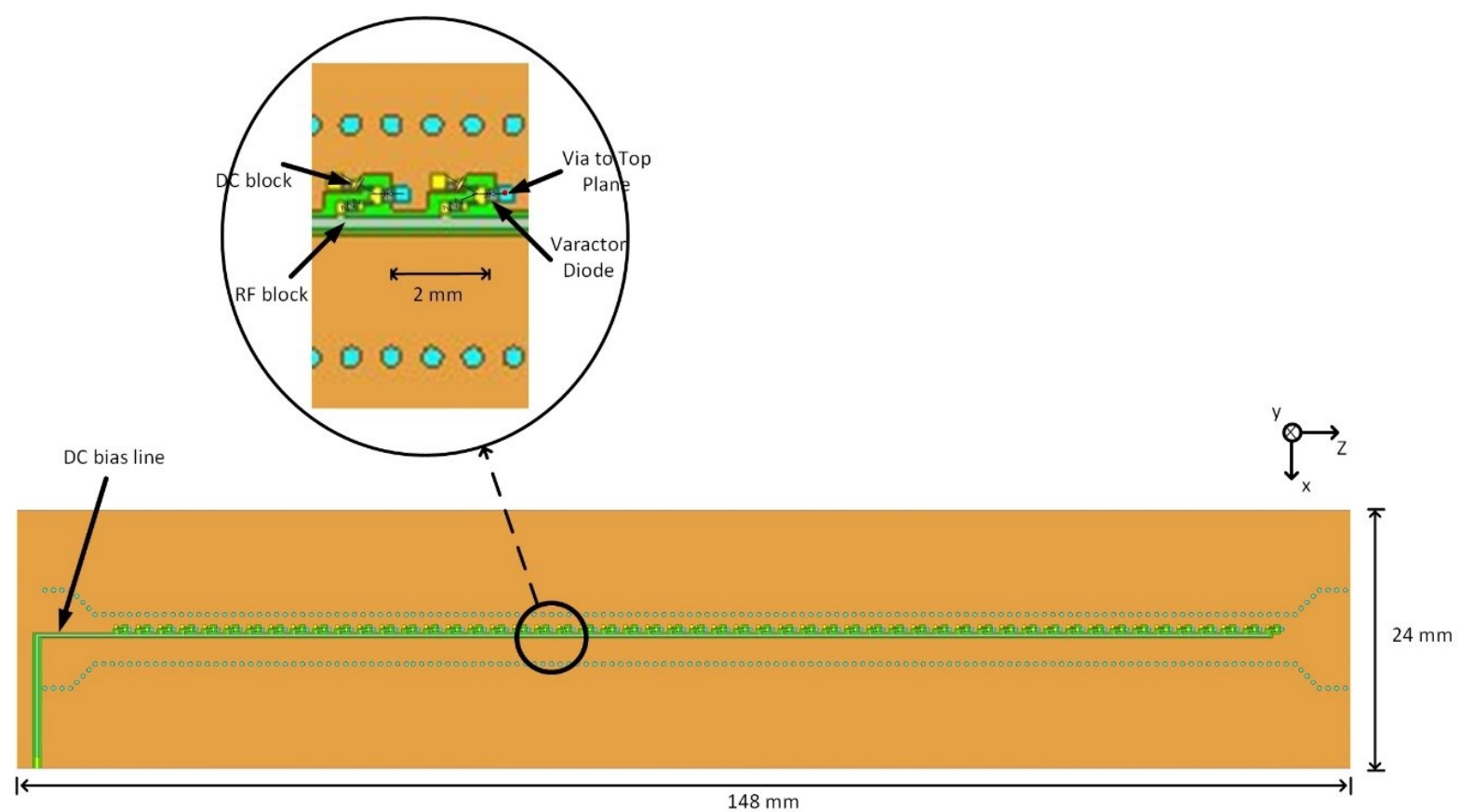

(d)

Figure 5.47. Schematic view of the proposed miniaturized reconfigurable antenna (cont'd). (d)

Bottom view.

To create isolation between the DC and RF signal, a gap surrounding the DC line was etched in the ground plane. This provided the required isolation to protect the DC source and RF signal generator. However, it resulted in higher SLL and lower gain because of the truncation of the ground plane. To minimize the undesired effect of the ground truncation while providing the required isolation, the gap size was chosen as $0.2 \mathrm{~mm}$ that is the smallest allowable gap size according to the fabrication technology. In addition, the location of the DC line was chosen such that the total gap length would be as small as possible. Several full-wave simulations were carried out to find the optimum location for the gap and DC line to minimize the effect of ground plane truncation, while providing enough isolation between DC and RF. Since the gap in the ground plane is much longer than the radiating slots at the top, it acts like a long slot. Therefore, radiation mostly occurs from the gap and the main-beam is located in the back hemisphere (i.e., $\theta<0^{\circ}$ ). 
The simulated S-parameters are reported in Fig. 5.48, indicating a return loss better than $10 \mathrm{~dB}$ in the frequency bands of $27.65-27.95 \mathrm{GHz}$ and $28.15-28.5 \mathrm{GHz}$. The insertion loss of the proposed antenna ranges from $28 \mathrm{~dB}$ to $41 \mathrm{~dB}$ indicating that a small amount of the input power reaches the output.

The gain radiation patterns of the proposed miniaturized antenna at $27.8 \mathrm{GHz}$ are presented in Fig. 5.49, which indicates $16^{\circ}$ beam-scanning through sweeping the varactor capacitance from $10 \mathrm{fF}$ to $1 \mathrm{pF}$. The peak realized gain of the proposed miniaturized SIW LWA is shown in Fig. 5.50. According to Fig. 5.50, the proposed miniaturized antenna radiates with the peak realized gain of $8.2 \pm 0.2 \mathrm{dBi}$ at $27.8 \mathrm{GHz}$. This is beneficial since gain of the antenna preferably should remain constant in beam-scanning applications.

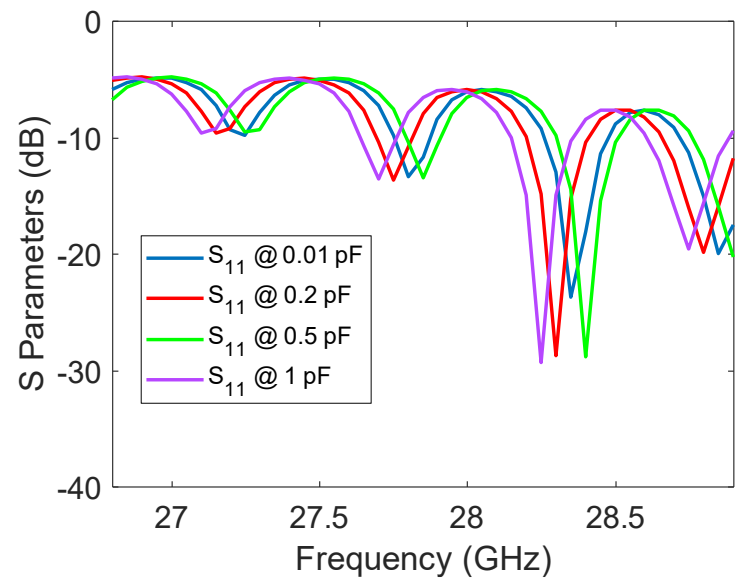

(a)

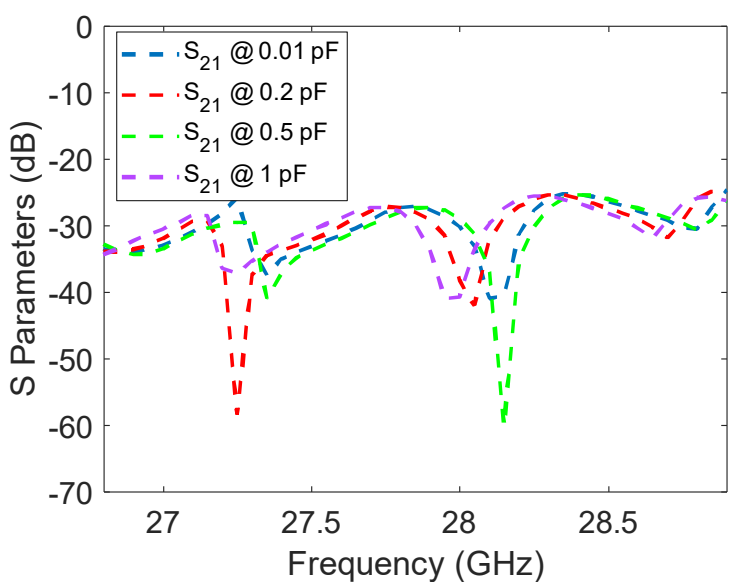

(b)

Figure 5.48. The simulated S-parameters of the proposed miniaturized reconfigurable LWA with longitudinal slots. (a) $S_{11}$, (b) $S_{21}$. 


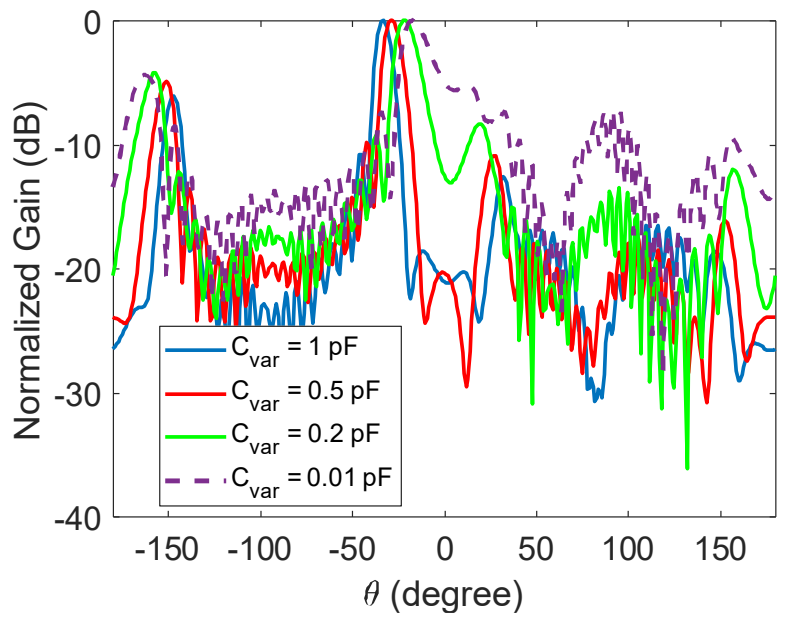

Figure 5.49. Radiation patterns of the proposed miniaturized reconfigurable SIW LWA composed of longitudinal slots.

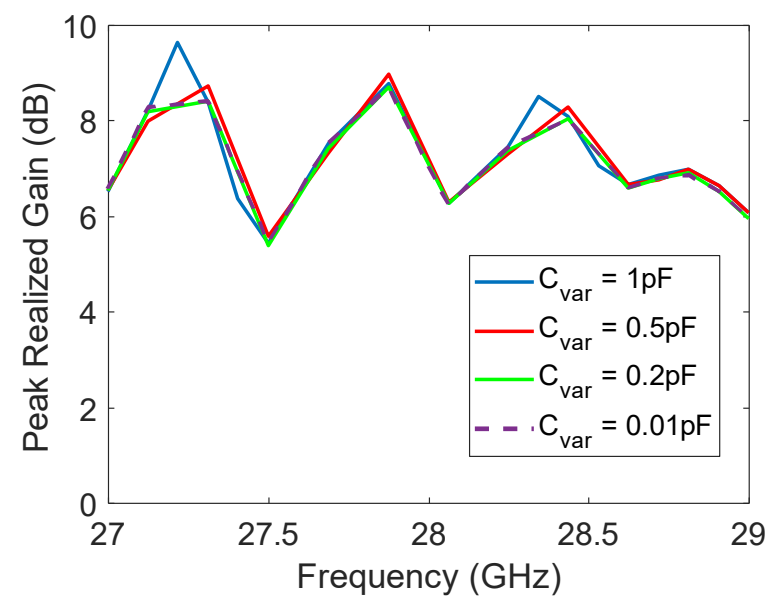

Figure 5.50. Peak realized gain of the proposed miniaturized reconfigurable SIW LWA for different varactor capacitance values.

Overall, electronic beam-scanning capability, low-profile, and small gain variation are among the features of the proposed reconfigurable antenna, making it a suitable candidate for the miniaturized $5 \mathrm{G}$ applications.

\subsubsection{Miniaturized Reconfigurable SIW LWA with Oblique Slots}

A miniaturized reconfigurable antenna composed of oblique slots is introduced in this section. The proposed LWA was designed on a two-layer SIW. The electronic beamscanning was achieved by introducing varactor diodes in oblique configuration on the backside of the antenna. The key features of the proposed design are the electronic beam- 
scanning using one varactor diode per cell and miniaturizing the antenna by integrating the bias circuitry into the ground plane. The proposed design was based on a single Rogers RT/Duroid 5880 substrate with 0.5 oz copper cladding, as illustrated in Fig. 5.51. The total length, width, and thickness of the miniaturized LWA are $95 \mathrm{~mm}, 24 \mathrm{~mm}$, and $0.127 \mathrm{~mm}$, respectively. Two rows of via fences were $4.6 \mathrm{~mm}$ apart to achieve single waveguide mode propagation at $29 \mathrm{GHz}$ according to (2.5) and (2.6). The proposed antenna consists of twenty-nine $1.6 \mathrm{~mm} \times 0.5 \mathrm{~mm}$ oblique slots to improve the beamscanning range and SLL. The spacing between consecutive cells was chosen as $2 \mathrm{~mm}$ to prevent higher order Floquet modes from propagation. The components are embedded into the ground in an oblique configuration that makes $45^{\circ}$ from the longitudinal axis.

As other antennas investigated in this dissertation, a tapered via fence in addition to the tapered microstrip transitions were used to improve the impedance matching. The input was connected to a k-connector, while the output was terminated with a $50 \Omega$ matched load. The bias circuitry was integrated into the RF ground plane to achieve a miniaturized structure and the ground plane was truncated to provide the required isolation between DC and RF. The truncation of the ground plane degrades the antenna performance, notably in terms of SLL and gain. Therefore, the bias circuitry was located to minimize the effect of ground plane truncation. The switches were located on the backside of the antenna and integrated into the ground plane. In order to bias the switch, the cathode of the varactor diode was connected to the DC line through an RF block, whereas the anode was connected to the upper plane through a via and grounded through via fences. Sweeping the bias voltage changes the surface current distribution, propagating fields, and radiation pattern. 


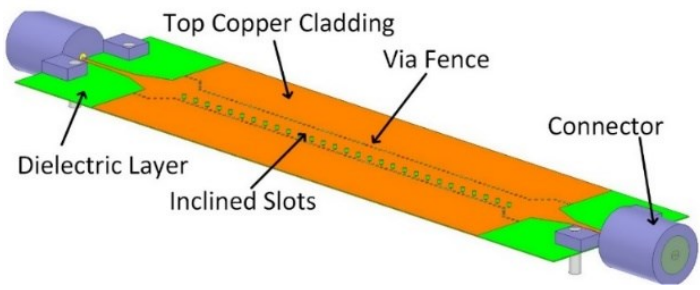

(a)

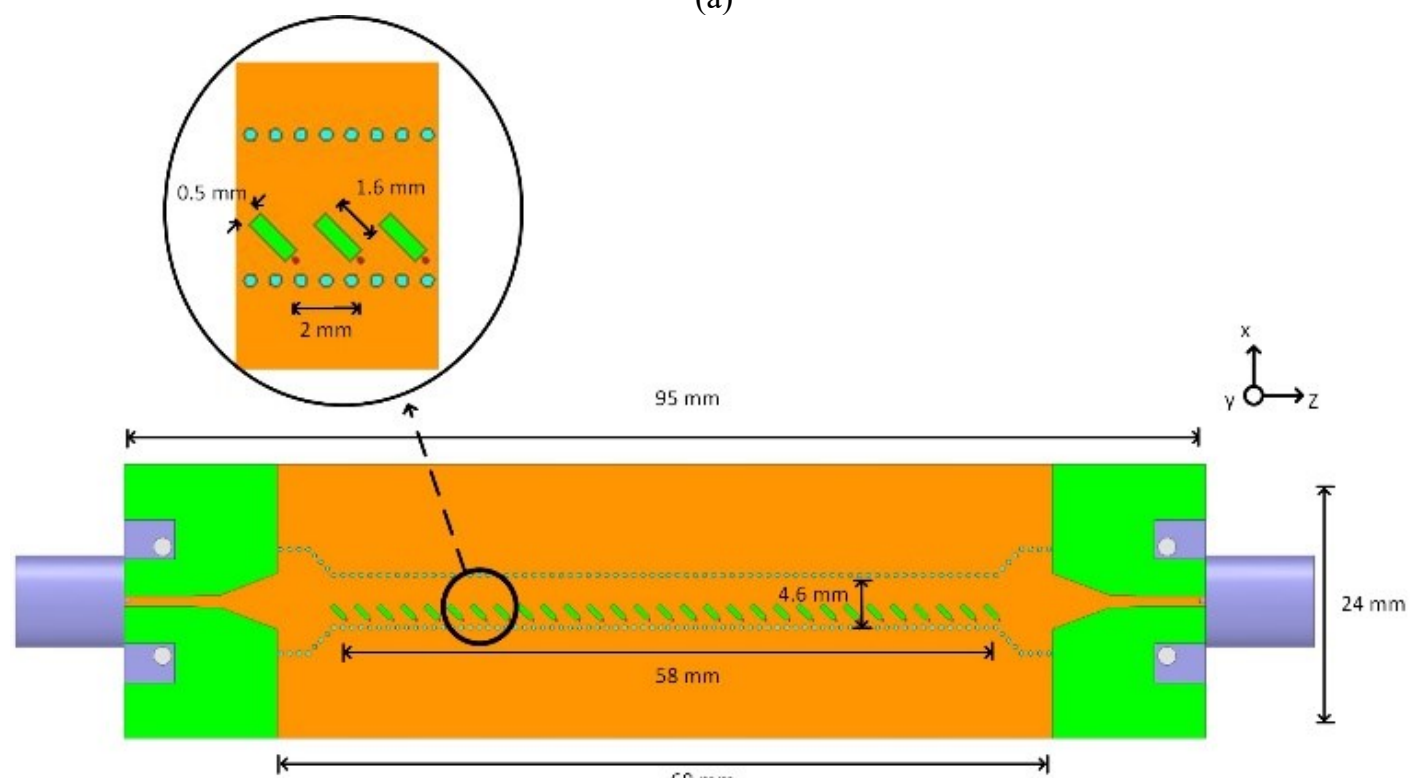

(b)

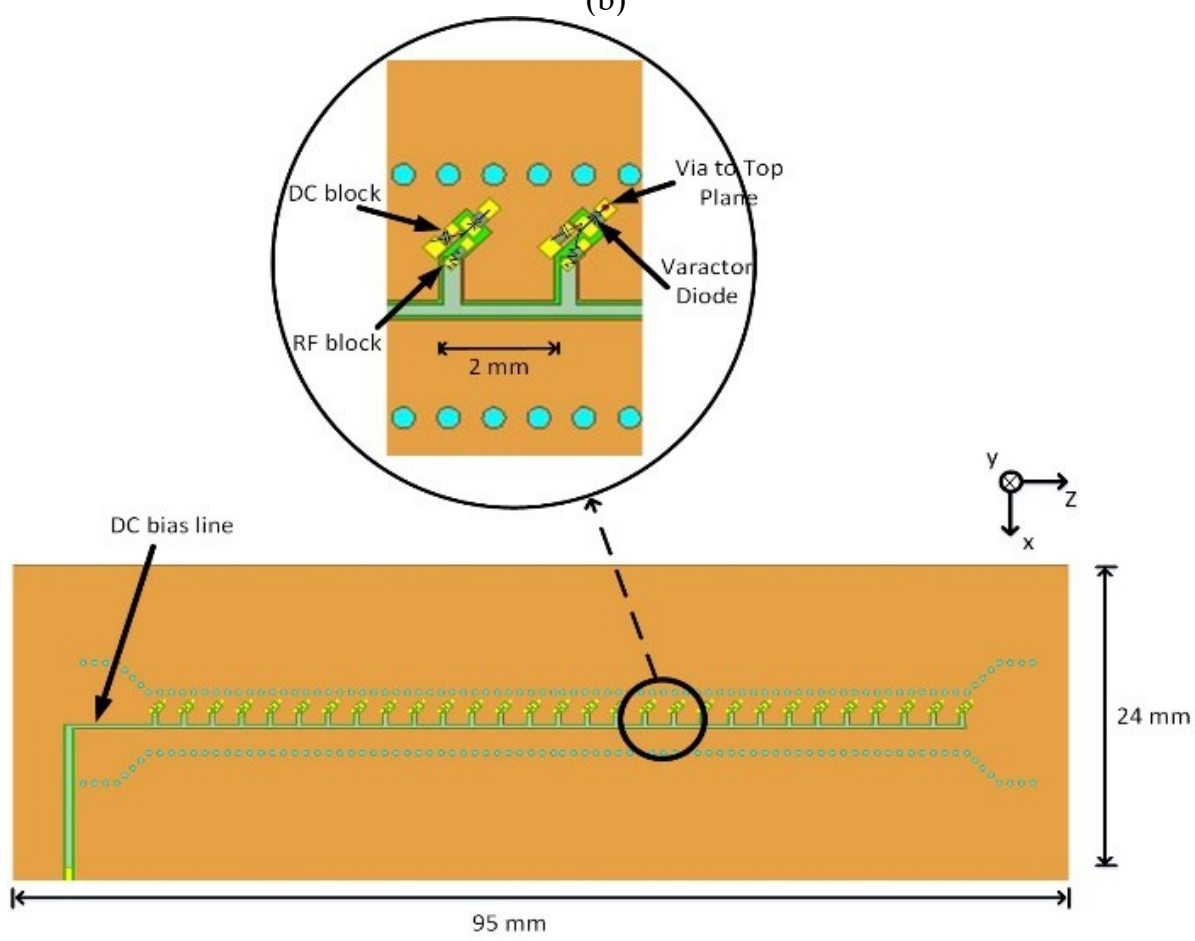

(c)

Figure 5.51. Schematic view of the proposed reconfigurable miniaturized LWA [116]. (a) 3D view, (b)

Top view, (c) Bottom view. 
The full-wave simulations have been carried out using Ansys HFSS 20.2. The simulated S-parameters are reported in Fig. 5.52, indicating a $10 \mathrm{~dB}$ impedance bandwidth of 28.6-29.7 GHz. The insertion loss of the proposed SIW LWA ranges from $30 \mathrm{~dB}$ to $40 \mathrm{~dB}$. The high insertion loss is because of the relatively high gain, small thickness of the substrate, and the loss of the RF blocks and the varactor diodes.

The normalized radiation patterns of the proposed miniaturized reconfigurable LWA at $29 \mathrm{GHz}$ in E-plane (YZ plane) are presented in Fig. 5.53, confirming the electronic beam-scanning by sweeping the capacitance from $200 \mathrm{fF}$ to $500 \mathrm{fF}$. The peak realized gain of the reconfigurable miniaturized antenna is presented in Fig. 5.54. According to Figs. 5.53 and 5.54, sweeping the varactor diode's capacitance results in an $18^{\circ}$ beamscanning with the peak realized gain of $12 \pm 0.2 \mathrm{dBi}$ at $29 \mathrm{GHz}$. The small variation in the peak realized gain indicates that the antenna radiates with an almost constant gain when sweeping the diodes' variable capacitance.

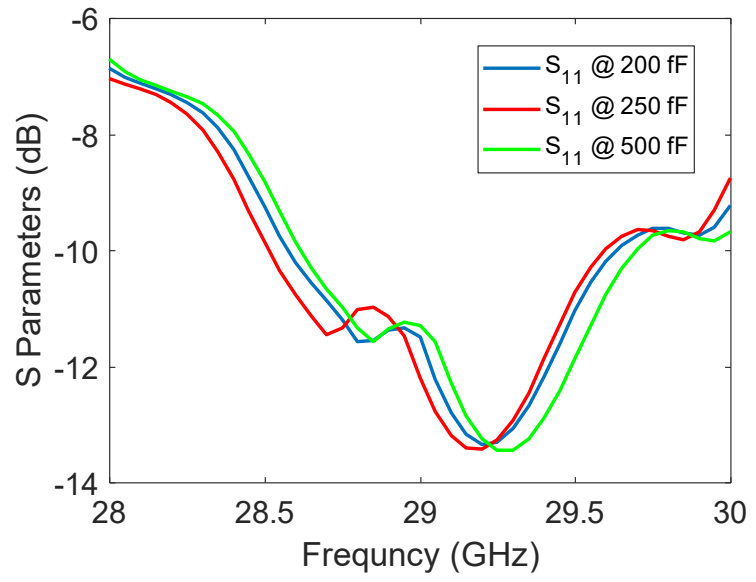

(a)

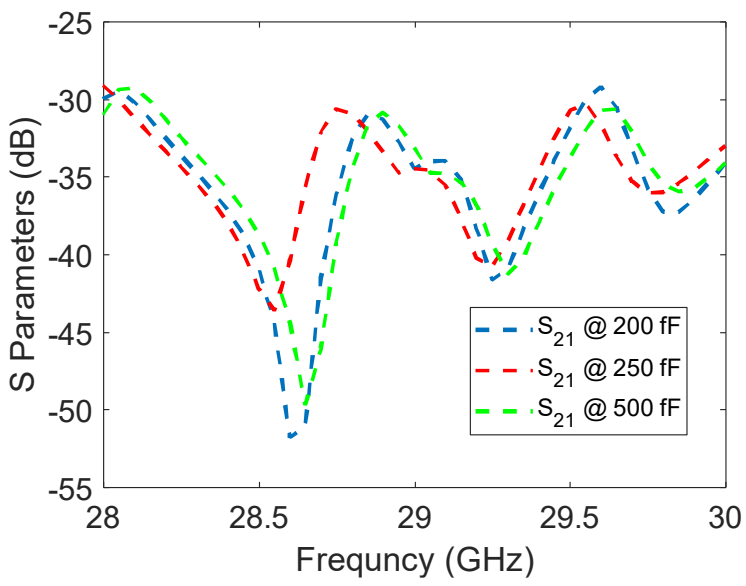

(b)

Figure 5.52. S Parameters of the proposed reconfigurable miniaturized LWA [116]. (a) $S_{11}$, (b) $S_{21}$. 


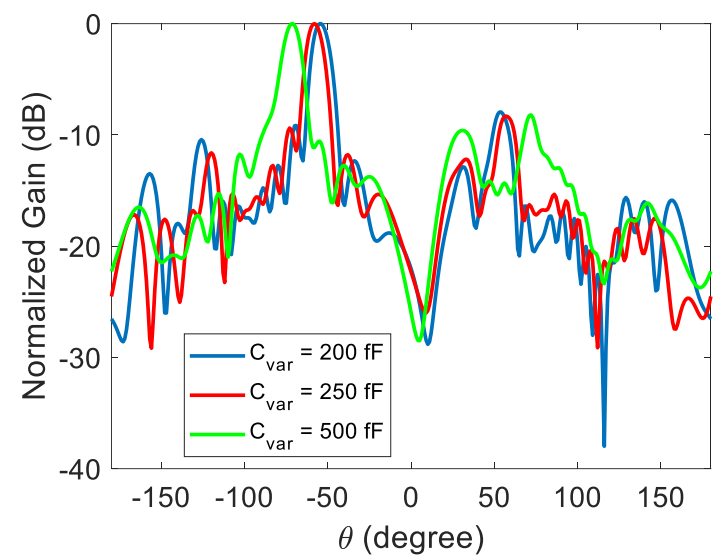

Figure 5.53. Normalized radiation pattern of the proposed miniaturized $L W A$ at $29 \mathrm{GHz}$ [116].

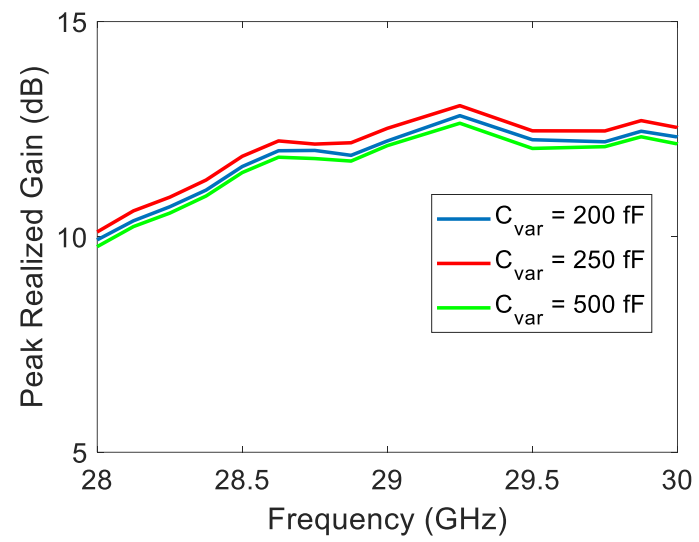

Figure 5.54. Peak realized gain of the proposed reconfigurable miniaturized LWA [116].

The gap size, pad sizes, and spacing between pads were chosen according to the current fabrication technology design rules. This limited the reconfigurable cell size and beam-scanning range. In the proposed reconfigurable antenna, for simplicity, all switches were biased uniformly using a single DC line. Non-uniform biasing of the varactors would result in a larger scanning range at the expense of increasing the complexity and the cost. Increasing the number of reconfigurable cells without changing their spacing may also lead to higher gain at the expense of the size and the cost. Overall, low-profile, electronic beam-scanning capability, low SLL, and relatively high gain are among the advantages of the proposed electronic beam-scanning antenna.

Unfortunately, fabrication of the proposed miniaturized LWAs on such thin substrates was not possible due to the fabrication technology limitations. The minimum required 
board thickness for fabricating such designs is $1.5 \mathrm{~mm}$ to boost the mechanical strength of the antenna and ensure the success of the assembly using automated machinery.

\subsubsection{Reconfigurable Gap SIW Antenna}

The proposed reconfigurable antenna consists of a SIW with two long parallel gaps adjacent to the via fences, as shown in Fig. 5.55. Introducing sets of varactor diodes over the gaps and tuning their capacitances lead to electronic beam-scanning. Switching disturbs the surface current and field distributions. When the switches are "on" (i.e., $500 \mathrm{fF}$ ), the surface current is grounded through the side via fences, and the antenna acts as a slotted SIW LWA. The proposed antenna acts as a semi-microstrip antenna when the switches are "off" (200fF) since surface current does not go to the ground. The introduced structure was first designed to achieve a hybrid SIW-microstrip antenna. It was expected that the proposed antenna would behave as SIW when the switches are "on" and as a microstrip when the switches are "off." However, this feature is only achieved if wide gaps are implemented since they provide sufficient spacing between side via fences and the main radiating section (i.e., slotted microstrip line). If wide gaps are present, with the switches "off," the propagating wave can be considered a quasi-TEM wave. However, increasing the width of the gaps requires either increasing the transversal spacing between via fences or reducing the length of the transverse slots. The former reduces the cutoff frequency of the propagating wave while the later results in lower leakage and radiation efficiency. As a result, increasing the width of the gaps beyond some point is not possible. Overall, the proposed design is a reconfigurable structure and potentially can be modified to be used as a hybrid SIW-microstrip antenna. 


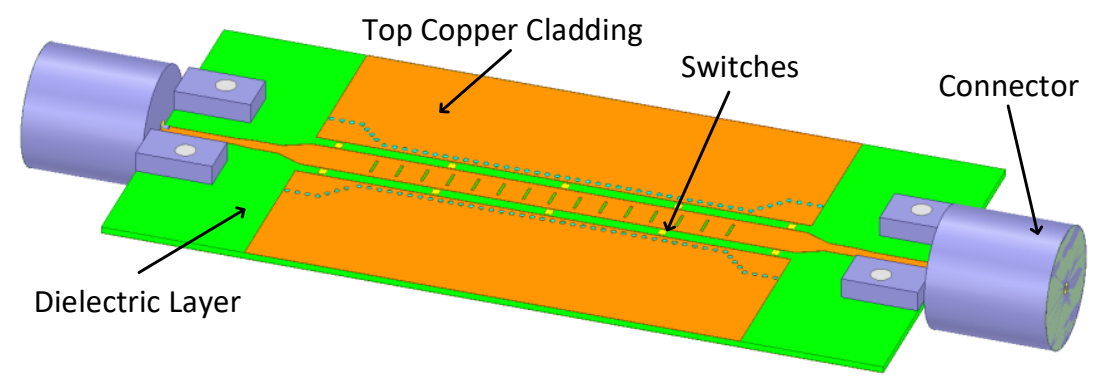

(a)

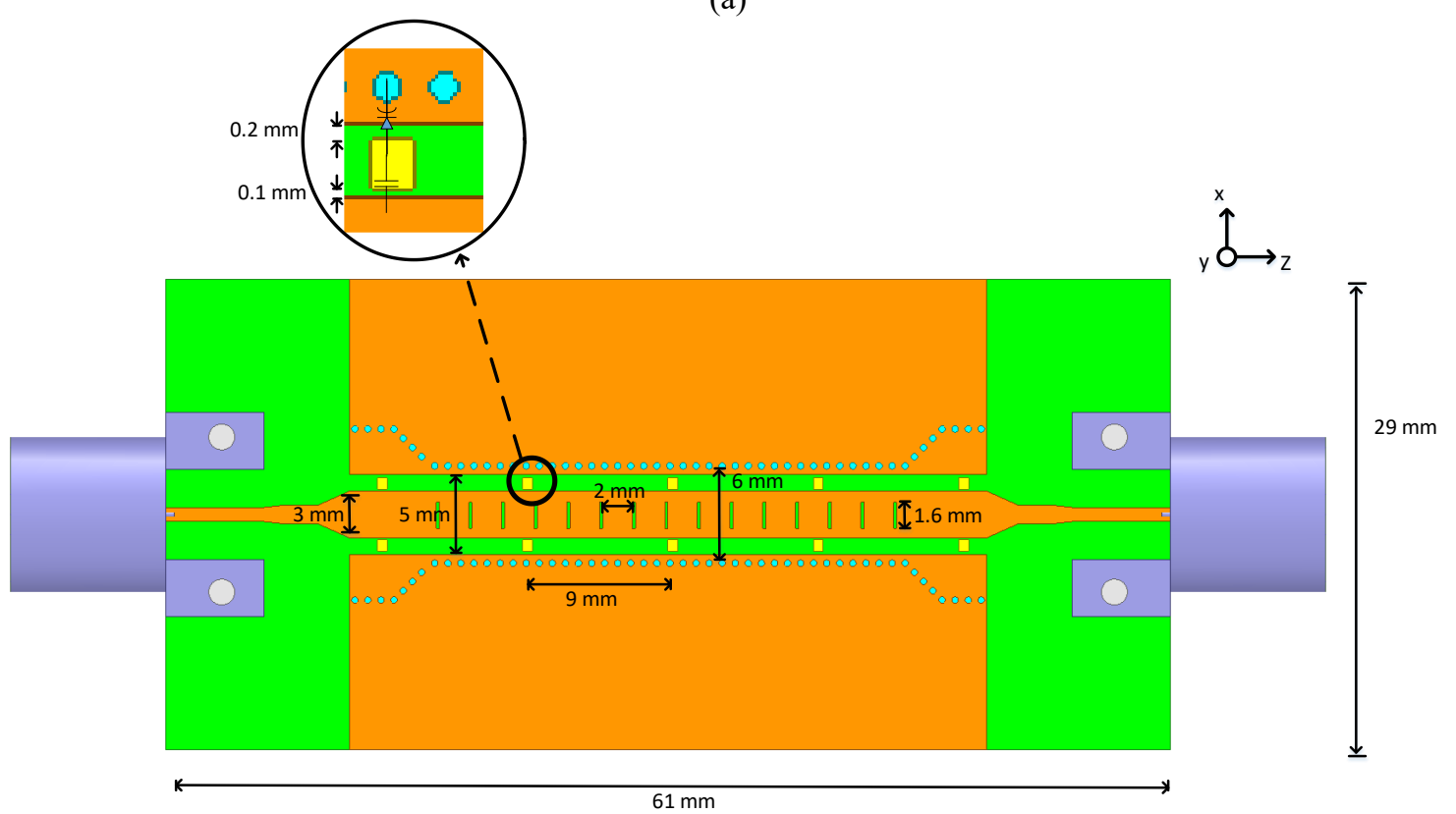

(b)

Figure 5.55. Schematic view of the proposed gap SIW LWA [117]. (a) 3D view, (b) top view.

The width, length, and thickness of the antenna are $29 \mathrm{~mm}, 61 \mathrm{~mm}$, and $1.1 \mathrm{~mm}$, respectively. The thickness of the antenna was chosen as $1.1 \mathrm{~mm}$ to reduce the conduction loss, while keeping the antenna compact. The radiating section of the antenna was obtained from (2.4) and consists of fifteen $1.6 \mathrm{~mm} \times 0.2 \mathrm{~mm}$ transversal slots. The two via fences of the SIW were $6 \mathrm{~mm}$ apart to achieve single mode propagation according to (2.5) and (2.6). The switches were located one guided wavelength $\left(\lambda_{\mathrm{g}}\right)$ apart, so that the signals that pass through the switches in each row will be in-phase. Sweeping the reverse bias voltage changes the propagating fields, current distribution, and consequently radiation pattern. In the proposed design, all switches are biased uniformly. 
The full-wave simulations have been carried out using Ansys HFSS v.20. The Sparameters are presented in Fig. 5.56 confirms a return loss better than $10 \mathrm{~dB}$ in all states from $28.4 \mathrm{GHz}$ to $29.2 \mathrm{GHz}$. The return loss and insertion loss at $28.5 \mathrm{GHz}$ vary from 10 $\mathrm{dB}$ to $25 \mathrm{~dB}$ and $10 \mathrm{~dB}$ to $40 \mathrm{~dB}$, respectively.

The radiation gain patterns of the antenna are presented in Fig. 5.57 indicate that sweeping the reverse bias voltage leads to the electronic beam-scanning with $7.4 \pm 0.3$ $\mathrm{dBi}$ peak realized gain. The proposed reconfigurable antenna scans about $30^{\circ}$ using the varactor capacitance ranging from $200 \mathrm{fF}$ to $500 \mathrm{fF}$. The proposed antenna is suitable for 5G mm-wave V2X applications since it provides a 3D image of the surrounding area by beam-scanning in principle planes.

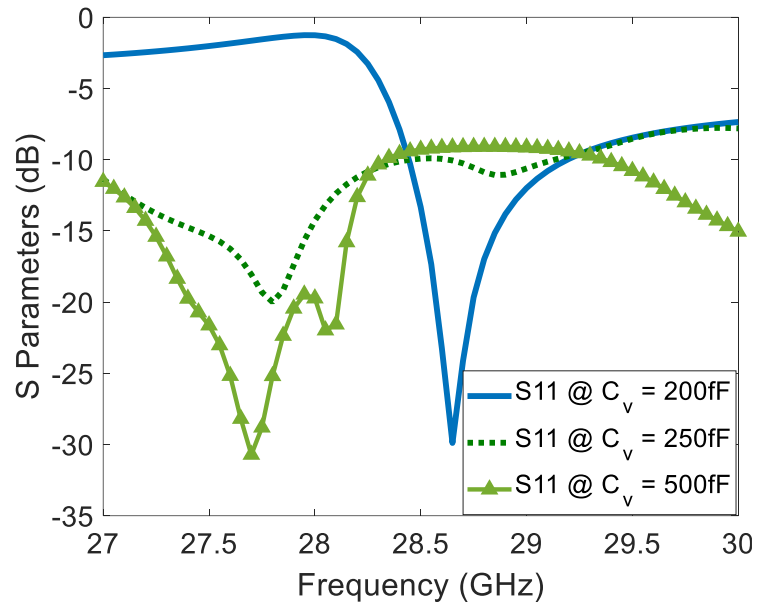

(a)

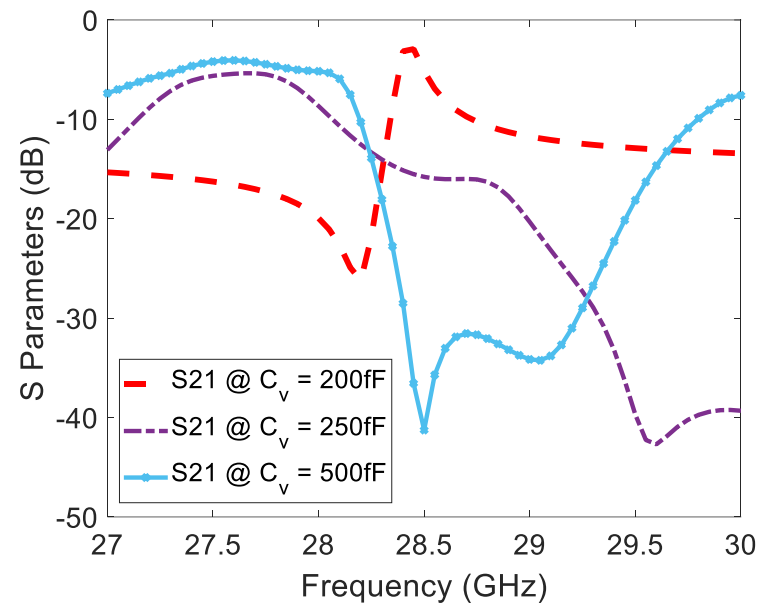

(b)

Figure 5.56. S Parameters of the proposed reconfigurable gap SIW LWA [117]. (a) $S_{11}$, (b) $S_{21}$. 


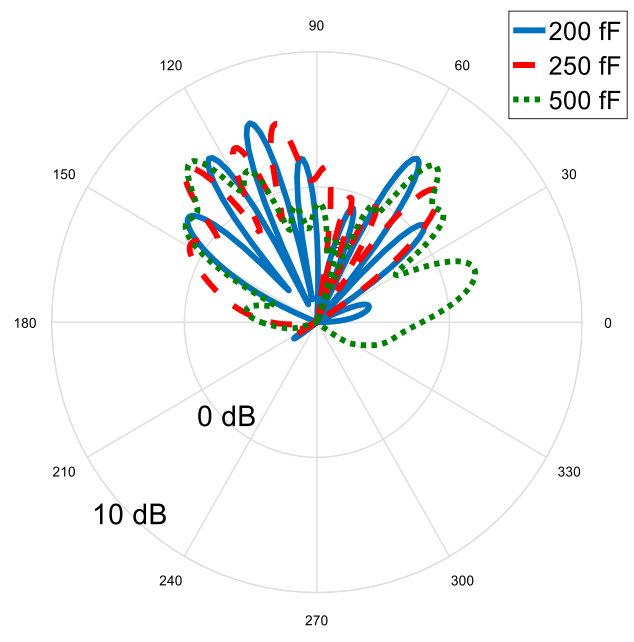

(a)

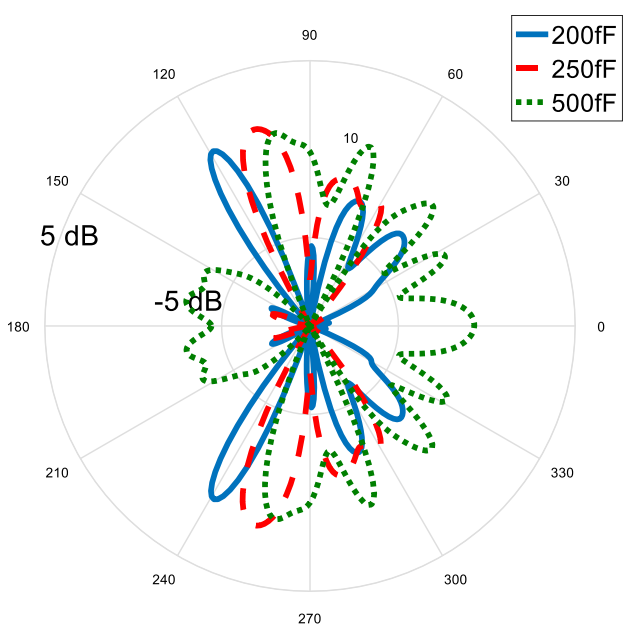

(b)

Figure 5.57. Radiation Patterns of the proposed reconfigurable gap SIW LWA at 28.7 GHz for different switching capacitance values (1) 200fF, (2) 250fF, and (3) 500fF. (a) E-plane (YZ plane), (b) XY-plane [117].

\subsection{Conclusion}

The electronic beam-scanning approach has been used in more applications compared to the frequency beam-scanning approach. Integrating sets of varactor diodes into the antenna and sweeping the reverse bias voltage leads to electronic beam-scanning that can partially compensate for undesired variations due to fabrication errors and variable environmental conditions. In this chapter, several different designs for achieving electronic beam-steering were investigated. First, a reconfigurable LWA with negative permittivity cells was designed. Then, a reconfigurable HMSIW with patches was investigated. Sets of reconfigurable HMSIW LWAs with circular cells and switches on the top and the backside were proposed subsequently. A reconfigurable SIW LWA with transverse slots was introduced afterward. Next, a reconfigurable SIW LWAs with longitudinal slots and switches on the backside was investigated through presenting the design, fabrication, and measurement. Later, two miniaturized SIW LWAs with 
components on the backside were proposed. Finally, a reconfigurable SIW antenna with controllable gaps was introduced.

In the proposed designs, a GaAs varactor diode was chosen as the tuning element to achieve beam-scanning. A $1 \mathrm{k} \Omega$ resistor and a $1 \mathrm{pF}$ capacitor were realized as an RF block and a DC block, respectively. The RF block was required for biasing the varactor diode and preventing the RF signal from entering the DC source. The DC block was required to create a path to the ground for the RF signal. Otherwise, due to the mismatch, large reflections occurred from the periodic cells that affected the radiation pattern undesirably. Tuning the capacitance of the GaAs varactor diode led to the variation of the series capacitance of each reconfigurable cell. This changed the impedance of the periodic cells and propagation constant that resulted in beam-scanning. In each of the proposed designs, electronic beam-scanning was achieved by tuning only one component per cell. This is a remarkable advantage of the proposed designs. One of the key features of the proposed designs is the implementation of reconfigurable cells as part of the main structure rather than adding them as extra sections to the structure. It is an essential benefit of the proposed antennas since in 5G communications device, the antenna dimensions must be as small as possible. Simple bias circuitry and ease of integration are among other benefits of the proposed structures.

Increasing the number of reconfigurable cells provides more choices for reconfiguring the antenna and increases the beam-scanning range. However, it adds to the complexity and the cost of the antenna. Increasing the length of the antenna also results in higher directivity at the expense of losing the compactness. Changing the period of slots and tapering the via fence and slots can also improve SLL. Varactor diodes for use at mm- 
wave frequency bands with lower loss and more extensive variable capacitance range would be useful for enhancing the gain and the beam-scanning range. However, achieving such a varactor diode is a practical challenge considering the state-of-the-art technology.

A comparison among all the antennas proposed in Chapter 5 is made in Table 5.6. Each of the reported LWAs has specific features that make it suitable for $5 \mathrm{G}$ mm-wave applications. A SIW LWA with offset longitudinal slots and miniaturized SIW LWA with oblique slots provide the lowest SLL and smallest gain variations. An HMSIW LWA with circular cells in V-shape topology on the backside and SIW with variable gap have the widest beam-scanning range with a small gain variation. A miniaturized SIW LWA with oblique slots provides the highest average gain.

In beam-scanning applications, the gain variation preferably should be below $3 \mathrm{~dB}$. Furthermore, the SLL should preferably be small to maximize the detection accuracy and robustness to interference. Overall, the following antennas are the most suitable candidates for 5G beam-scanning applications (e.g., V2X communications and radar systems) due to their compactness, wide beam-scanning range, and small gain variations:

1. HMSIW LWA with circular slots in V-shape topology,

2. HMSIW LWA with circular cells in V-shape configuration on the backside,

3. SIW LWA with offset longitudinal slots,

4. SIW LWA with offset longitudinal cells on the backside, and

5. Miniaturized SIW LWA with oblique slots and switches on the backside.

The proposed reconfigurable SIW LWAs with switches located on the backside are suitable for radar system' horizontal detection due to the radiation near the endfire. In 
contrast, the rest are suitable for azimuthal detection because of the radiation near the broadside. A combination of the proposed reconfigurable antennas can be implemented in

Table 5.6. A comparison among proposed reconfigurable antennas in Chapter 5 in terms of scanning range, peak gain, the absolute value of SLL (ASLL), and antenna length.

\begin{tabular}{|c|c|c|c|c|}
\hline Antenna Type & Scanning Range & Peak Gain (dBi) & ASLL (dB) & Antenna Length \\
\hline $\begin{array}{l}\text { HMSIW LWA } \\
\text { with negative } \varepsilon_{r} \\
\text { cells } \\
\end{array}$ & $45^{\circ}$ & $6.5 \pm 5.5$ & $12 \pm 6$ & $4 \times \lambda$ \\
\hline $\begin{array}{l}\text { HMSIW LWA } \\
\text { with patches }\end{array}$ & $50^{\circ}$ & $2.4 \pm 2.2$ & $2.25 \pm 1.25$ & $6 \times \lambda$ \\
\hline $\begin{array}{l}\text { HMSIW LWA } \\
\text { with circular slots } \\
\text { and transversal } \\
\text { switching }\end{array}$ & $15^{\circ}$ & $5.7 \pm 3$ & $6 \pm 2$ & $6 \times \lambda$ \\
\hline $\begin{array}{l}\text { HMSIW LWA } \\
\text { with circular slots } \\
\text { in V-shape } \\
\text { topology }\end{array}$ & $20^{\circ}$ & $7.65 \pm 0.55$ & $3 \pm 2$ & $6 \times \lambda$ \\
\hline $\begin{array}{l}\text { HMSIW LWA } \\
\text { with circular cells } \\
\text { in V-shape } \\
\text { configuration on } \\
\text { the backside } \\
\end{array}$ & $29^{\circ}$ & $8.2 \pm 0.6$ & $5 \pm 0.6$ & $6 \times \lambda$ \\
\hline $\begin{array}{l}\text { SIW LWA with } \\
\text { transverse slots } \\
\text { and uniform } \\
\text { switching } \\
\end{array}$ & $35^{\circ}$ & $4.6 \pm 4.5$ & $4.25 \pm 3.75$ & $6 \times \lambda$ \\
\hline $\begin{array}{l}\text { SIW LWA with } \\
\text { transverse slots } \\
\text { and non-uniform } \\
\text { switching }\end{array}$ & $81^{\circ}$ & $3.8 \pm 3.3$ & $2.25 \pm 1.75$ & $6 \times \lambda$ \\
\hline $\begin{array}{c}\text { SIW LWA with } \\
\text { offset longitudinal } \\
\text { slots }\end{array}$ & $27^{\circ}$ & $4.7 \pm 0.2$ & $8.5 \pm 0.5$ & $9 \times \lambda$ \\
\hline $\begin{array}{l}\text { SIW LWA with } \\
\text { offset longitudinal } \\
\text { slots and switches } \\
\text { on the backside }\end{array}$ & $25^{\circ}$ & $9 \pm 0.8$ & $7 \pm 1.6$ & $9 \times \lambda$ \\
\hline Gap SIW LWA & $30^{\circ}$ & $7.4 \pm 0.3$ & $2 \pm 1$ & $6 \times \lambda$ \\
\hline $\begin{array}{l}\text { Miniaturized SIW } \\
\text { LWA with } \\
\text { longitudinal slots } \\
\text { and switches on } \\
\text { the backside }\end{array}$ & $16^{\circ}$ & $8.2 \pm 0.2$ & $5 \pm 1.5$ & $14 \times \lambda$ \\
\hline $\begin{array}{l}\text { Miniaturized SIW } \\
\text { LWA with oblique } \\
\text { slots and switches } \\
\text { on the back }\end{array}$ & $18^{\circ}$ & $12 \pm 0.2$ & $9 \pm 0.5$ & $9 \times \lambda$ \\
\hline
\end{tabular}


radar systems to enable the capability of switching between horizontal and vertical scanning. The proposed reconfigurable antennas with main-beams radiating near endfire are suitable for blind-spot monitoring, while the ones that radiate near the broadside are useful for vehicle cruise control and detecting obstacles on the road. 


\section{Chapter 6: Conclusion}

\subsection{Summary}

The motivations and objectives of this dissertation along with the proposed methodologies were discussed in Chapter 1. A comprehensive literature review regarding the current challenges in 5G mm-wave applications, LWA, SIW/HMSIW, LTCC, and electronic beam-scanning antennas was presented in Chapter 2. Then several LWAs were proposed in Chapters 3, 4, and 5 to reduce SLL, increase gain, and achieve electronic beam-steering capability. The simulations and measurement results indicate that the proposed antennas are suitable candidates for $5 \mathrm{G}$ mm-wave applications.

Several antenna technologies were proposed to enhance current trends in $5 \mathrm{G} \mathrm{mm}$ wave applications such as autonomous vehicles, V2X communications, point-to-point communications, and radar systems. An HMSIW/SIW-based LWA was chosen as the backbone of the proposed antennas since it can achieve desired beam-scanning range without using a complex feed network. The low-profile and integration capability are among additional benefits of the proposed designs. The SLL, gain, and the beamscanning range of the LWA were improved by implementing different approaches in this dissertation.

The main objective of the antennas proposed in Chapter 3 is the SLL reduction. This is achieved by tapering the aperture and modifying the feed transition. Reducing interference in the wireless network is crucial. This can be achieved by improving SLL. An HMSIW LWA with tapered aperture was proposed in Chapter 3 for point to point communications. Tapering the aperture led to a small SLL and frequency beam-squint that is useful for the targeted $5 \mathrm{G}$ mm-wave applications, which requires an almost fixed 
beam antenna with low interference. The proposed antenna radiates with a measured gain of $10.6 \mathrm{dBi}$ and SLL of $-11.2 \mathrm{~dB}$ at $28.5 \mathrm{GHz}$, which meets the required specifications of 5G mm-wave point to point communication systems [7], [18], [22], [25].

Furthermore, reducing SLL enhances detection accuracy that is important in radar systems. Modifying the feed transition improved the SLL. A SIW LWA fed by tapered CPW was proposed in Chapter 3 for radar systems detecting targets on the horizon and monitoring the blind spots. The SLL, gain, and beam-scanning range of the proposed SIW antenna are $-11.4 \mathrm{~dB}, 6.1 \mathrm{dBi}$, and $16^{\circ}$, respectively. Hence, the proposed SIW LWA meets the necessary specifications of the mm-wave $5 \mathrm{G}$ radar and blind spot monitoring systems [18], [22], [25], [27].

The main goal of the proposed method in Chapter 4 is the gain improvement of a compact antenna. This is achieved by embedding cavities into the structure. The gain improvement is required to partly compensate for the path loss in wireless networks. The peak realized gain and beam-scanning range of the proposed LTCC HMSIW-based LWA are $7.6 \mathrm{dBi}$ and $3^{\circ}$, respectively, which meet the specifications for mm-wave $5 \mathrm{G}$ point to point communication systems [7], [18], [22], [25], [27]. The compactness of the proposed LTCC-based antenna makes it a suitable choice for 5G miniaturized wireless communication devices. Fabricating the proposed antenna on PCB is more complicated compared to LTCC. Moreover, LTCC has a superior packaging tolerance that is useful for miniaturized applications.

The key purpose of the designs introduced in Chapter 5 is the enhancement of the beam-scanning range, which is achieved by implementing different reconfigurable cells containing GaAs varactor diodes, RF blocks, and DC blocks. To enable electronic beam- 
scanning capability, several reconfigurable antennas were designed. First, a reconfigurable antenna was developed based on periodic cells with negative permittivity. Unfortunately, as discussed in Chapter 5, such an antenna could not be manufactured due to fabrication technology limitations. Different types of reconfigurable HMSIW antenna with patches and circular slots were designed. It was found that the V-shape topology leads to the best performance in terms of scanning range and gain. Next, reconfigurable SIW LWAs with transverse and longitudinal slots were proposed. The reconfigurable HMSIW LWA with reconfigurable cells in V-shape topology and SIW LWA with longitudinal slots were fabricated as proof of concept. To miniaturize the reconfigurable antenna, bias circuitry was integrated into the RF ground. SIW LWAs with oblique and longitudinal slots were designed using the proposed miniaturization technique. Finally, a reconfigurable SIW LWA with gaps along the side via fences was investigated.

The measured beam-scanning range and peak realized gain at center frequency of the most suitable reconfigurable antennas for the mm-wave beam-scanning applications are:

1. $29^{\circ}$ and $8.2 \pm 0.6 \mathrm{~dB}$ for the HMSIW LWA composed of switches in the V-shape configuration on the backside,

2. $25^{\circ}$ and $9 \pm 0.8 \mathrm{dBi}$ for the SIW LWA composed of longitudinal cells with switches on the backside,

3. $20^{\circ}$ and $7.65 \pm 0.55 \mathrm{dBi}$ for the HMSIW LWA composed of circular slots in the Vshape configuration,

4. $27^{\circ}$ and $4.7 \pm 0.2 \mathrm{dBi}$ for the SIW LWA composed of longitudinal slots,

5. $18^{\circ}$ and $12 \pm 0.2 \mathrm{dBi}$ for the miniaturized SIW LWA composed of oblique slots. 
These antennas meet the specifications for $5 \mathrm{G}$ beam-scanning applications such as radar systems and V2X communications [24], [26], as stated in Chapter 1. Implementing electronic beam-scanning antennas are also useful for covering several users in a dense area and compensating for environmental variations. A combination of the proposed antennas would enable switching between horizontal and vertical scanning in radar systems because some of the proposed antennas radiate near endfire while the rest radiate near broadside.

Electronic beam-scanning in the proposed LWAs was achieved by implementing sets of varactor diodes at the top or the backside of the antenna. By placing the switches on the backside of the antenna, the unwanted blockage effects of the components on the radiation pattern were reduced. Assembling the components on the backside also simplified the fabrication since more space was available. Implementing the reconfigurable cells as part of the main radiating section rather than adding additional sections to the antenna is a key advantage of the proposed designs. Electronic beamscanning was achieved by tuning only one varactor (i.e., series capacitor) per cell in all the proposed reconfigurable designs. This is another advantage of the proposed designs since achieving beam-steering using fewer switches results in lower loss, cost, and complexity.

In summary, the important contributions of this doctoral research can be categorized as follows:

1. The SLL of HMSIW LWA was reduced by tapering the side aperture. This reduced the sensitivity to interference. Implementing the proposed method led to 
SLL of $-13.8 \mathrm{~dB}$ and $-11.2 \mathrm{~dB}$ in the upper hemisphere and full space, respectively.

2. The SLL of SIW LWA was improved by modifying the feed transition. This was achieved by implementing tapered CPW as feed transition, which led to $-11.4 \mathrm{~dB}$ SLL and improved the detection accuracy.

3. The gain of a compact HMSIW LWA was improved by implementing cavities in an LTCC structure. This led to $7.6 \mathrm{dBi}$ of gain and partially compensated for the path loss in a wireless network.

4. Electronic beam-scanning in an HMSIW LWA was achieved using reconfigurable metamaterial cells. An array of reconfigurable cells with negative permittivity replaced the via fence. Sweeping the bias voltage of the switches was similar to shifting the via fence artificially. This enabled the reconfigurability of the structure, which led to electronic beam-scanning in the range of $45^{\circ}$ at $28.5 \mathrm{GHz}$.

5. Implementing sets of reconfigurable cells in HMSIW-based LWAs resulted in electronic beam-scanning with relatively small gain variations. Different topologies including reconfigurable circular cells in uniform and V-shape configuration were designed to accommodate 5G beam-scanning applications, such as V2X communications. For instance, the HMSIW LWA composed of switches in the $\mathrm{V}$-shape configuration achieved the beam-scanning range of $20^{\circ}$ with the peak gain of $7.65 \pm 0.55 \mathrm{dBi}$.

6. Implementing sets of reconfigurable cells in transverse and longitudinal configurations in SIW LWAs also led to electronic beam-scanning. For example, the SIW LWA composed of longitudinal cells achieved the beam-scanning range 
of $27^{\circ}$ with the peak gain of $4.7 \pm 0.2 \mathrm{dBi}$. Assembling switches on the backside of the antenna reduced the blockage effect of the components on the radiation pattern.

7. Integrating the bias circuitry into the ground plane resulted in the miniaturization of the electronic beam-scanning antennas. It should be noted that the fabrication technology limited the realization of a few of the proposed antennas.

\subsection{Conclusion}

Overall, the antennas proposed, analyzed, simulated, and measured in this doctoral dissertation meet the required specifications for different $5 \mathrm{G}$ applications in terms of gain, beam-scanning range, and SLL, as stated in Chapter 1 and reported in [7], [18], [22]-[27]. The compactness, medium gain, low SLL, and beam-scanning capability of the antennas studied make them suitable candidates for $5 \mathrm{G}$ mm-wave applications.

The conclusion of this doctoral research can be listed as follows:

1. In an LWA, there is a trade-off between return loss, peak gain, SLL, and beamscanning range due to the correlation between attenuation constant $(\alpha)$ and phase constant $(\beta)$. This limited the performance of the LWAs proposed in this dissertation.

2. A SIW/HMSIW is a suitable structure for realizing an LWA. Fabrication of a SIW/HMSIW is cheaper and easier than waveguide. However, errors in the locations of the via fences and the presence of airgaps result in discrepancies between the measured and simulated results. For example, $0.15 \mathrm{~mm}$ error in $W$ resulted in $9 \mathrm{~dB}$ discrepancies in the SLL of an HMSIW LWA at $28.5 \mathrm{GHz}$, as shown in Fig. 3.14. 
3. LTCC is an expensive technology suitable for realizing miniaturized structures due to high packaging tolerance. However, the thermal expansion of the layers causes discrepancies between the measured and simulated results.

4. The low-profile structure is achieved at the expense of high loss, which degrades the gain. Thin substrates may bend under the physical stress that degrades the antenna performance. This is a real concern, which needs to be addressed. For example, bending the antenna proposed in Chapter 4 reduced the gain by $4 \mathrm{dBi}$ at $28.5 \mathrm{GHz}$, as presented in Fig. 4.22 .

5. HMSIW is more compact than SIW. However, controlling the leakage in an HMSIW LWA is more difficult than a SIW LWA due to the presence of the long side aperture.

6. Reducing the reconfigurable cell size results in a more negative $\varepsilon_{\mathrm{r}}$. Hence, smaller cells confine the fields more effectively and are more suitable for implementation in the electronic beam-scanning antenna. However, the cell size is limited by the fabrication technology. As an example, the reconfigurable cell presented in Fig. 5.1(b) with the size of $\lambda_{0} / 20$ achieved $\varepsilon_{r}=-38.79$ at $28.5 \mathrm{GHz}$ for $\mathrm{V}_{\text {bias }}=-25 \mathrm{~V}$, as shown in Fig. 5.2(a). However, realizing such a small structure using the current technology was not possible.

7. Electronic beam-scanning antennas suffer from the additional losses introduced by the switches, RF blocks, and DC blocks. These losses can be reduced by designing custom-made electronic components. 
8. Assembling the electronic components on a thin substrate is a practical challenge. This is because the board may bend, and it is difficult to assemble the components in their correct locations.

9. The state-of-the-art fabrication technology limits the realization of the antennas studied here. Future technologies may enable them to be realized practically.

\subsection{Future Work}

Fully programmable beam-steerable antennas open the door to many interesting applications such as $5 \mathrm{G}$ automated vehicles. Designing reconfigurable cells with a negative dielectric constant that can be fabricated using the current technology is the first step to be taken to achieve a fully programmable antenna. Optimizing the feed distribution to the reconfigurable cells is another crucial step to achieve this goal. A few different types of new reconfigurable cells for achieving more negative $\varepsilon_{\mathrm{r}}$ and increasing beam-scanning range are presented in Fig. 6.1. the proposed structures are designed on Rogers RT/Duroid 5880 with a thickness of $0.127 \mathrm{~mm}$ to achieve low loss and lowprofile structures. The proposed reconfigurable cells shown in Figs. 6.1(a) and 6.1(b), make use of common ground to reduce the size. The symmetry of the cells also results in a more uniform field distribution. The proposed antenna in Fig. 6.1(c) uses three arrays of reconfigurable cells at each side of radiating transverse slots. Sweeping the bias voltage of the embedded diodes in the reconfigurable cells can lead to variations in the antenna width, cut-off frequency, and consequently beam-scanning. The proposed designs are works in progress and can be realized in the future by using more recent fabrication technologies. 
Realization of the proposed reconfigurable cells in Gallium Nitride (GaN), GaAs, and silicon-based technologies is another interesting topic for future research. This results in the miniaturization of the proposed design and improving the packaging tolerance. In addition, it is possible to bias the varactor diodes wirelessly. This leads to the removal of bias circuitry and eases the fabrication procedure.
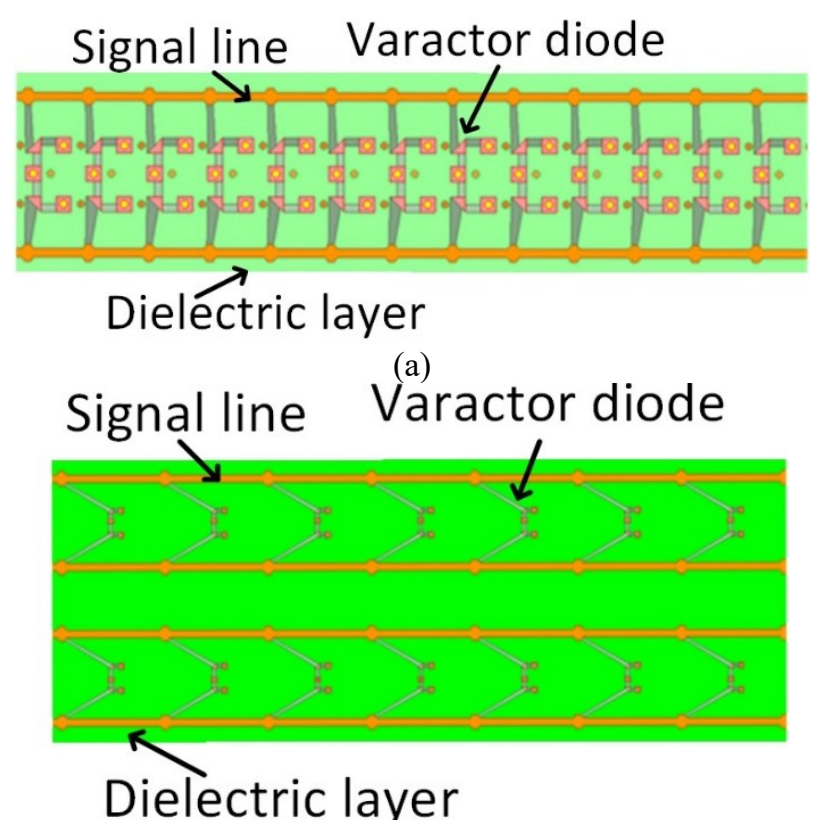

(b)

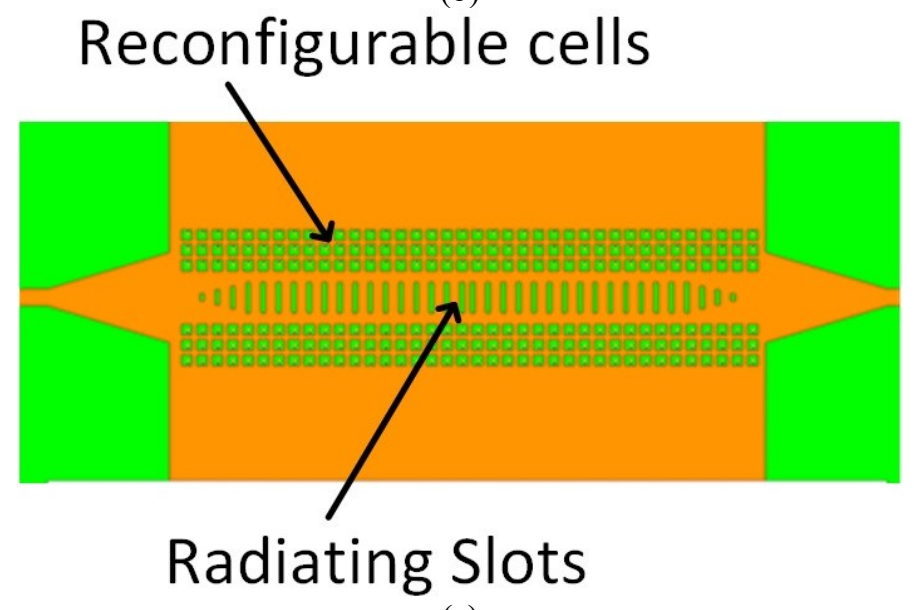

(c)

Figure 6.1. Different types of new reconfigurable cells currently under investigation. (a) linear array of reconfigurable cells with common ground, (b) planar array of reconfigurable cells with common ground, (c) LWA with reconfigurable cells at sides of transverse slots. 
All the proposed antennas in this dissertation were single-element antennas. Placing them in an array configuration may improve SLL, gain, and beam-scanning range. Doing so provides more freedom in the antenna design at the expense of increasing the cost and complexity of the feed network and bias circuitry. A few arrays of HMSIW LWAs with the tapered apertures, shown in Fig. 6.2, are currently under investigation. The proposed arrays are designed on a $0.127 \mathrm{~mm}$ thick Rogers RT/Duroid 5880. In each array, one connector is connected to the signal generator while the others are terminated by matched loads.
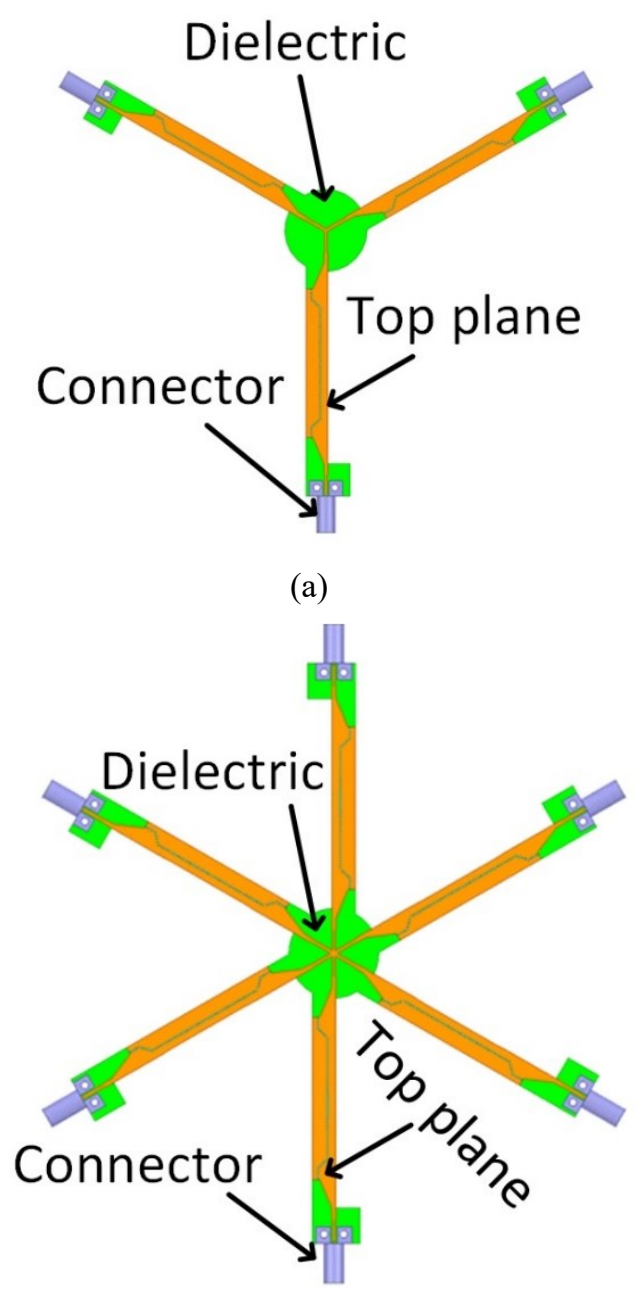

(b)

Figure 6.2. Few arrays of HMSIW LWA with tapered side apertures. (a) Y-shape topology, (b) Starshape topology. 
Realization of the proposed designs in a conformal topology is another exciting area for the extension of this work. The conformal antenna is popular in the car and airplane industry since the antenna can be mounted directly on the car bumper and airplane fuselage.

Extending the ideas presented in this dissertation to antennas for future $6 \mathrm{G}$ systems and higher frequency bands such as $\mathrm{W}$-band is future work that can be done in this thrilling research area.

\subsection{Contributions to Research Established by Published Papers}

[1] N. Javanbakht, B. Syrett, and R. Amaya, "Leaky-wave antenna based on modified aperture half-mode substrate integrated waveguide," IEEE Int. Symp. Antennas Propag. USNC-URSI Radio Science Meeting, Boston, 2018.

[2] N. Javanbakht, B. Syrett, R. Amaya, and J. Shaker, "Low temperature co-fired ceramic half-mode substrate integrated waveguide leaky-wave antenna," IEEE Int. Symp. Antenna Tech. Applied Electromagnetics, Waterloo, 2018.

[3] N. Javanbakht, B. Syrett, R. Amaya, and J. Shaker, "Miniaturized reconfigurable antenna based on half-mode substrate integrated waveguide," IEEE Int. Systems Conf., Miami, 2019.

[4] N. Javanbakht, B. Syrett, R. Amaya, and J. Shaker, "Electronically steerable quasiuniform leaky-wave antenna based on substrate integrated waveguide," Canadian Conf. Elec. Comp. Eng., Edmonton, 2019.

[5] N. Javanbakht, B. Syrett, R. Amaya, and J. Shaker, "Electric beam-steering metamaterial leaky-wave antenna," Int. Conf. Electromagnetics Advanced Applications, Granada, 2019. 
[6] N. Javanbakht, B. Syrett, R. Amaya, and J. Shaker, "Non-Uniform Steerable LeakyWave Antenna Based on Substrate Integrated Waveguide," IEEE Int. Symp. Phased Array Sys. Tech., Boston, 2019.

[7] N. Javanbakht, B. Syrett, R. Amaya, and J. Shaker, "Electric Beam-Steering LeakyWave Antenna for Space Communications," IEEE Int. Conf. Wireless Space Extreme Environment, Ottawa, 2019.

[8] N. Javanbakht, B. Syrett, R. Amaya, and J. Shaker, "Electronic beam-scanning leakywave antenna based on delta shape half-mode substrate integrated waveguide," European Conf. Antennas Propag., Copenhagen, 2020.

[9] N. Javanbakht, B. Syrett, R. Amaya, and J. Shaker, "Reducing side-lobe level of surface-mounted printed leaky-wave antenna," European Conf. Antennas Propag., Copenhagen, 2020.

[10] N. Javanbakht, B. Syrett, R. Amaya, and J. Shaker, "Reconfigurable antenna based on gap substrate integrated waveguide," IEEE Int. Symp. Antennas Propag. USNC-URSI Radio Science Meeting, Montreal, 2020.

[11] N. Javanbakht, B. Syrett, R. Amaya, and J. Shaker, "Electronic steerable slotted leaky-wave antenna," IEEE Int. Symp. Antennas Propag. USNC-URSI Radio Science Meeting, Montreal, 2020.

[12] N. Javanbakht, B. Syrett, R. Amaya, and J. Shaker, "Side-lobe level reduction of half-mode substrate integrated waveguide leaky-wave antenna," IEEE Trans. Antennas Propag., vol. 68, Nov. 2020. 
[13] N. Javanbakht, B. Syrett, R. Amaya, and J. Shaker, "A Tapered CPW fed leakywave antenna based on substrate integrated waveguide with reduced side-lobe level," Wiley Int. Jour. RF Microw. Comp. Eng., Feb. 2021.

[14] N. Javanbakht, B. Syrett, R. Amaya, and J. Shaker, “A compact cavity-based leakywave antenna in a low temperature co-fired ceramic process with improved performance," IEEE Access, vol. 9, Feb. 2021.

[15] N. Javanbakht, B. Syrett, R. Amaya, and J. Shaker, "Reconfigurable SIW-based leaky-wave antenna composed of longitudinal slots," IEEE Antennas. Wirel. Propag. Lett., submitted for publication.

[16] N. Javanbakht, B. Syrett, R. Amaya, and J. Shaker, "Fixed frequency beam-scanning HMSIW-based leaky-wave antenna composed of circular slots in V-shape configuration," IEEE Access, vol. 9, Mar. 2021.

[17] N. Javanbakht, B. Syrett, R. Amaya, and J. Shaker, "Miniaturized reconfigurable SIW-based leaky-wave antenna loaded with inclined slot," IEEE Int. Symp. Antennas Propag. USNC-URSI Radio Science Meeting, Singapore, submitted for publication, 2021.

[18] N. Javanbakht, B. Syrett, R. Amaya, and J. Shaker, "Low-profile electronic beamscanning leaky-wave antenna composed of longitudinal cells," IEEE Int. Symp. Antenna Tech. Applied Electromagnetics, Winnipeg, submitted for publication, 2021.

[19] N. Javanbakht, B. Syrett, R. Amaya, and J. Shaker, "A review of reconfigurable leaky-wave antenna," IEEE Access, submitted for publication, 2021.

\subsection{Acknowledgement}

I would like to thank Department of Electronics at Carleton University, PolyGram lab at Polytechnique de Montreal, ETS lab, CMR Summit Company, and ETI Tech Company for the measurements and fabrication of the proposed antennas in this dissertation. 


\section{References}

[1] M. Shafi et al., "5G: A tutorial overview of standards, trials, challenges, deployment, and practice," IEEE J. Sel. Areas Commun., vol. 35, no. 6, pp. 12011221, 2017, doi: 10.1109/JSAC.2017.2692307.

[2] C. Deng, D. Liu, B. Yektakhah, and K. Sarabandi, "Series-fed Beamsteerable Millimeter-wave Antenna Design with Wide Spatial Coverage for 5G Mobile Terminals," IEEE Trans. Antennas Propag., vol. PP, no. c, p. 1, 2020, doi: 10.1109/TAP.2019.2963583.

[3] J. G. Andrews et al., "What will 5G be?," IEEE J. Sel. Areas Commun., vol. 32, no. 6, pp. 1065-1082, 2014, doi: 10.1109/JSAC.2014.2328098.

[4] Th. S. Rappaport, et al., "Overview of millimeter wave communications for fifth-generation $(5 \mathrm{G})$ wireless networks with a focus on propagation models," IEEE Trans. Antennas Propag., vol. 65, no. 12, pp. 6213-6230, Dec. 2017.

[5] W. Hong, K. Baek, and S. Ko, "Millimeter-wave 5G antennas for smartphones: overview and experimental demonstration," IEEE Trans. Antennas Propag., vol. 65, no. 12, pp. 6250-6261, Dec. 2017.

[6] H. Kong, Z. Wen, Y. Jing and M. Yau, "Midfield Over-the-Air Test: A New OTA RF Performance Test Method for 5G Massive MIMO Devices," IEEE Transactions on Microwave Theory and Techniques, vol. 67, no. 7, pp. 28732883, July 2019, doi: 10.1109/TMTT.2019.2912369.

[7] T. Tuovinen, N. Tervo and A. Pärssinen, "Analyzing 5G RF System Performance and Relation to Link Budget for Directive MIMO," IEEE Transactions on Antennas and Propagation, vol. 65, no. 12, pp. 6636-6645, Dec. 
2017, doi: 10.1109/TAP.2017.2756848.

[8] A. Ghosh, A. Maeder, M. Baker, and D. Chandramouli, "5G Evolution: A View on 5G Cellular Technology Beyond 3GPP Release 15," IEEE Access, vol. 7, pp. 127639-127651, 2019, doi: 10.1109/ACCESS.2019.2939938.

[9] M. Agiwal, A. Roy, and N. Saxena, "Next generation 5G wireless networks: A comprehensive survey," IEEE Commun. Surv. Tutorials, vol. 18, no. 3, pp. 1617-1655, 2016, doi: 10.1109/COMST.2016.2532458.

[10] L. Miller, 5 G for Dummies, Wiley, New York: John Wiley, 2017.

[11] W. S. H. M. W. Ahmad et al., "5G Technology: Towards Dynamic Spectrum Sharing Using Cognitive Radio Networks," IEEE Access, vol. 8, pp. 14460-14488, 2020, doi: 10.1109/ACCESS.2020.2966271.

[12] Y. N. R. Li, B. Gao, X. Zhang, and K. Huang, "Beam Management in Millimeter-Wave Communications for 5G and beyond," IEEE Access, vol. 8, pp. 13282-13293, 2020, doi: 10.1109/ACCESS.2019.2963514.

[13] W. Hong et al., "Multibeam Antenna Technologies for 5G Wireless Communications," IEEE Trans. Antennas Propag., vol. 65, no. 12, pp. 62316249, 2017, doi: 10.1109/TAP.2017.2712819.

[14] L. Reichardt, J. Maurer, T. Fügen, and T. Zwick, "Virtual drive: A complete V2X communication and radar system simulator for optimization of multiple antenna systems," Proc. IEEE, vol. 99, no. 7, pp. 1295-1310, 2011, doi: 10.1109/JPROC.2011.2124430.

[15] Y. Nakayama, T. Nishio, D. Hisano, and K. Maruta, "Small Cells Enabled by Crowdsourced Radio Units Mounted on Parked Vehicles for Smart City," 
IEEE Access, vol. 8, pp. 17986-17998, 2020, doi: 10.1109/ACCESS.2020.2967747.

[16] E. Kampert, C. Schettler, R. Woodman, P. A. Jennings, and M. D. Higgins, "Millimeter-wave communication for a last-mile autonomous transport vehicle," IEEE Access, vol. 8, pp. 8386-8392, 2020, doi: 10.1109/ACCESS.2020.2965003.

[17] D. Garcia-Roger, E. E. González, D. Martín-Sacristán and J. F. Monserrat, "V2X Support in 3GPP Specifications: From 4G to 5G and Beyond," IEEE Access, vol. 8, pp. 190946-190963, 2020, doi: 10.1109/ACCESS.2020.3028621.

A. Bazzi, C. Campolo, A. Molinaro, A. O. Berthet, B. M. Masini and A. Zanella, "On Wireless Blind Spots in the C-V2X Side link," IEEE Transactions on Vehicular Technology, vol. 69, no. 8, pp. 9239-9243, Aug. 2020, doi: 10.1109/TVT.2020.3001074.

[19] M. Mozaffari, A. T. Z. Kasgari, W. Saad, M. Bennis, and M. Debbah, "Beyond 5G with UAVs: Foundations of a 3D Wireless Cellular Network," IEEE Trans. Wirel. Commun., vol. 18, no. 1, pp. 357-372, 2019, doi: 10.1109/TWC.2018.2879940.

[20] Sh. Zhang, I. Syrytsin, and F. Pedersan, "Compact beam steerable antenna array with two passive parasitic elements for 5G mobile terminals at $28 \mathrm{GHz}$," IEEE Trans. Antennas Propag., vol. 66, no. 10, pp. 5193-5203, Jul. 2018.

Y. Yashchyshyn, et al., "28 GHz Switched-beam antenna based on S-PIN diodes for 5G mobile communications," IEEE Antennas Wireless Propag. Lett., vol. 17, no. 2, pp. 225-228, Feb. 2018. 
"Fifth Generation Antennas: A Comprehensive Review of Design and Performance Enhancement Techniques," IEEE Access, vol. 8, pp. 163568163593, 2020, doi: 10.1109/ACCESS.2020.3020952.

[23] "Fixed Radio Systems: Characteristics and requirements for point-topoint equipment and antennas; Part 4: Antennas", ETSI EN 302 217-4, v.2.0.3, 2016.

P. W. Futter and J. Soler, "Antenna design for 5g communications," 2017 Sixth Asia-Pacific Conference on Antennas and Propagation (APCAP), 2017, pp. 1-3, doi: 10.1109/APCAP.2017.8420649.

M. S. Khan, et.al., "Eight-Element Compact UWB-MIMO/Diversity Antenna with WLAN Band Rejection for 3G / 4G/ 5G Communications," IEEE Open Journal of Antennas and Propagation, vol. 1, pp. 196-206, 2020, doi: 10.1109/OJAP.2020.2991522.

[26] N. Ojaroudiparchin, Ming Shen and G. F. Pedersen, "Wide-scan phased array antenna fed by coax-to-microstriplines for 5G cell phones," $201621 s t$ International Conference on Microwave, Radar and Wireless Communications (MIKON), Krakow, 2016, pp. 1-4, doi: 10.1109/MIKON.2016.7492064.

[27] A. Araghi, M. Khalily, P. Xiao and R. Tafazolli, "Study on the Location of mmWave Antenna for the Autonomous Car's Detection and Ranging Sensors," 2020 14th European Conference on antenna and propagation, Copenhagen, 2020.

[28] J. L. Volakis, Antenna Engineering Handbook, 2nd ed. New York: 
McGraw-Hill, 2007.

[29] C. A. Balanis, Modern Antenna Handbook, 1st ed. New York: John Wiley, 2008.

[30] F. B. Gross, Frontiers in antennas : next generation design \& engineering. New York: McGraw-Hill, 2012.

[31] F. Monticone and A. Alù, "Leaky-wave theory, techniques, and applications: From microwaves to visible frequencies," Proc. IEEE, vol. 103, no. 5, pp. 793-821, 2015, doi: 10.1109/JPROC.2015.2399419.

[32] F. Mesa, D. R. Jackson, and M. J. Freire, "Evolution of leaky modes on printed-circuit lines," IEEE Trans. Microw. Theory Tech., vol. 50, no. 1 I, pp. 94104, 2002, doi: 10.1109/22.981253.

[33] Y. Li, Q. Xue, E. K. N. Yung, and Y. Long, "The periodic half-width microstrip leaky-wave antenna with a backward to forward scanning capability," IEEE Trans. Antennas Propag., vol. 58, no. 3, pp. 963-966, 2010, doi: 10.1109/TAP.2009.2039304.

[34] K. M. Mak, K. K. So, H. W. Lai, and K. M. Luk, "A magneto-electric dipole leaky wave antenna for millimeter wave application," IEEE Trans. Antennas and Propag., vol. 65, pp. 6395-6402, Dec. 2017.

[35] F. Xu and K. Wu, "Guided-wave and leakage characteristics of substrate integrated waveguide," IEEE Trans. Microw. Theory Tech., vol. 53, no. 1, pp. 6672, 2005, doi: 10.1109/TMTT.2004.839303.

[36] Z. Kordiboroujeni and J. Bornemann, "Designing the Width of Substrate Integrated Waveguide Structures," IEEE Microw. Wireless Comp. Lett., vol. 23, 
no. 10, pp. 518-520, Oct. 2013.

[37] Y. Cassivi, L. Perregrini, P. Arcioni, M. Bressan, K. Wu, and G. Conciauro, "Dispersion characteristics of substrate integrated rectangular waveguide," IEEE Microw. Wirel. Components Lett., vol. 12, no. 9, pp. 333-335, 2002, doi: 10.1109/LMWC.2002.803188.

[38] D. Deslandes and K. Wu, "Accurate Modeling, Wave Mechanisms, and Design Considerations of a Substrate Integrated Waveguide," IEEE Trans. Microw. Theory Tech., vol. 54, no. 6, pp. 2516-2526, 2006, doi: 10.1109/TMTT.2006.875807.

M. Salehi and E. Mehrshahi, "A closed-form formula for dispersion characteristics of fundamental SIW mode," IEEE Microw. Wirel. Components Lett., vol. 21, no. 1, pp. 4-6, 2011, doi: 10.1109/LMWC.2010.2088114.

M. Bozzi, A. Georgiadis, and K. Wu, "Review of substrate-integrated waveguide circuits and antennas," IET Microwaves, Antennas Propag., vol. 5, no. 8, pp. 909-920, 2011, doi: 10.1049/iet-map.2010.0463.

H. Uchimura, T. Takenoshita, and M. Fujii, "Development of a "laminated waveguide," IEEE Trans. Microw. Theory Tech., vol. 46, no. 12 PART 2, pp. 2438-2443, 1998, doi: 10.1109/22.739232.

[42] J. Hirokawa and M. Ando, "Single-layer feed waveguide consisting of posts for plane tem wave excitation in parallel plates," IEEE Trans. Antennas Propag., vol. 46, no. 5, pp. 625-630, 1998, doi: 10.1109/8.668903. D. Deslandes and $\mathrm{K}$. Wu, "Integrated microstrip and rectangular waveguide in planar form," IEEE Microw. Wirel. Components Lett., vol. 11, no. 
2, pp. 68-70, 2001, doi: 10.1109/7260.914305.

S. Rezaee and M. Memarian, "Analytical Study of Open-Stopband Suppression in Leaky-Wave Antennas," IEEE Antennas Wirel. Propag. Lett., vol. 19, no. 2, pp. 363-367, 2020, doi: 10.1109/LAWP.2019.2963798.

R. Ranjan and J. Ghosh, "SIW-Based Leaky-Wave Antenna Supporting Wide Range of Beam Scanning Through Broadside," IEEE Antennas Wirel. Propag. Lett., vol. 18, no. 4, pp. 606-610, 2019, doi: 10.1109/LAWP.2019.2897836.

[46] W. Zhou, J. Liu, and Y. Long, "Investigation of Shorting Vias for Suppressing the Open Stopband in an SIW Periodic Leaky-Wave Structure," IEEE Trans. Microw. Theory Tech., vol. 66, no. 6, pp. 2936-2945, 2018, doi: 10.1109/TMTT.2018.2818140.

Y. F. Wu and Y. J. Cheng, "Proactive Conformal Antenna Array for NearField Beam Focusing and Steering Based on Curved Substrate Integrated Waveguide," IEEE Trans. Antennas Propag., vol. 67, no. 4, pp. 2354-2363, 2019, doi: 10.1109/TAP.2019.2891725. Y. J. Cheng, W. Hong, K. Wu, and Y. Fan, "Millimeter-wave substrate integrated waveguide long slot leaky-wave antennas and two-dimensional multibeam applications," IEEE Trans. Antennas Propag., vol. 59, no. 1, pp. 4047, 2011, doi: 10.1109/TAP.2010.2090471.

D. Zheng, Y. L. Lyu, and K. Wu, "Longitudinally Slotted SIW LeakyWave Antenna for Low Cross-Polarization Millimeter-Wave Applications," IEEE Trans. Antennas Propag., vol. 68, no. 2, pp. 656-664, 2020, doi: 
10.1109/TAP.2019.2940469.

[50] A. M. Malekshah, M. S. Majedi, and A. R. Attari, "Improved design of a SIW long slot leaky wave antenna with low SLL," IET Microwaves, Antennas Propag., vol. 13, no. 1, pp. 112-117, 2019, doi: 10.1049/iet-map.2018.5443. J. Liu, D. R. Jackson, and Y. Long, "Substrate integrated waveguide (SIW) leaky-wave antenna with transverse slots," IEEE Trans. Antennas Propag., vol. 60, no. 1, pp. 20-29, 2012, doi: 10.1109/TAP.2011.2167910.

J. Liu, D. R. Jackson, Y. Li, C. Zhang, and Y. Long, "Investigations of SIW leaky-wave antenna for endfire-radiation with narrow beam and sidelobe suppression," IEEE Trans. Antennas Propag., vol. 62, no. 9, pp. 4489-4497, 2014, doi: 10.1109/TAP.2014.2336659.

N. Javanbakht, M. S. Majedi, and A. R. Attari, "Thinned Array Inspired Quasi-Uniform Leaky-Wave Antenna with Low Side-Lobe Level," IEEE Antennas Wirel. Propag. Lett., vol. 16, 2017, doi: 10.1109/LAWP.2017.2756990. Y. Mohtashami and J. Rashed-Mohassel, “A butterfly substrate integrated waveguide leaky-wave antenna," IEEE Trans. Antennas Propag., vol. 62, no. 6, pp. 3384-3388, 2014, doi: 10.1109/TAP.2014.2311464.

Q. Zhang, Q. Zhang, H. Liu, and C. H. Chan, "Dual-band and dualpolarized leaky-wave antenna based on slotted SIW," IEEE Antennas Wirel. Propag. Lett., vol. 18, no. 3, pp. 507-511, 2019, doi: 10.1109/LAWP.2019.2895339. S. Sengupta, et.al., "A Cross-Shaped 2-D Periodic Leaky-Wave Antenna," IEEE Trans. Antennas Propag., vol. 68, no. 3, pp. 1289-1301, 2020, doi: 
10.1109/TAP.2019.2948524.

D. K. Karmokar, Y. J. Guo, P. Y. Qin, S. L. Chen, and T. S. Bird, "Substrate integrated waveguide-based periodic backward-to-forward scanning leaky-wave antenna with low cross-polarization," IEEE Trans. Antennas Propag., vol. 66, no. 8, pp. 3846-3856, 2018, doi: 10.1109/TAP.2018.2835502.

S. L. Chen, D. K. Karmokar, Z. Li, P. Y. Qin, R. W. Ziolkowski, and Y. J.

Guo, "Circular-polarized substrate-integrated-waveguide leaky-wave antenna with wide-angle and consistent-gain continuous beam scanning," IEEE Trans. Antennas Propag., vol. 67, no. 7, pp. 4418-4428, 2019, doi: 10.1109/TAP.2019.2911398.

[59] Y. Geng, J. Wang, Z. Li, Y. Li, M. Chen, and Z. Zhang, "Dual-Beam and Tri-Band SIW Leaky-Wave Antenna with Wide Beam Scanning Range including Broadside Direction," IEEE Access, vol. 7, pp. 176361-176368, 2019, doi: 10.1109/ACCESS.2019.2957763.

[60] Y. L. Lyu et al., "Leaky-Wave Antennas Based on Noncutoff Substrate Integrated Waveguide Supporting Beam Scanning from Backward to Forward," IEEE Trans. Antennas Propag., vol. 64, no. 6, pp. 2155-2164, 2016, doi: 10.1109/TAP.2016.2550054.

[61] N. Javanbakht, B. Syrett, R. Amaya, and J. Shaker, "Reducing side-lobe level of surface mounted printed leaky-wave antenna," Proc. 14th Eur. Conf. Antennas Propagation, EUCAP 2020, Copenhagen, 2020.

[62] N. Javanbakht, B. Syrett, R. Amaya, and J. Shaker, “A Tapered CPW fed leaky-wave antenna based on substrate integrated waveguide with reduced side- 
lobe level," Wiley Int. Jour. RF Microw. Comp. Eng., Feb. 2021.

[63] J. Xu, W. Hong, H. Tang, Zh. Kuai, and K. Wu, "Half-mode substrate integrated waveguide leaky-wave antenna for millimeter-wave applications," IEEE Antennas Wireless Propag. Lett., vol. 7, pp. 85-88, 2008.

[64] N. Trong, Th. Kaufmann, and Ch. Fumeaux, “A Semi-analytical solution for a tapered half-mode substrate integrated waveguide with application to rapid antenna optimization," IEEE Trans. Antennas Propag., vol. 62, no. 6, pp. 31893200, Jun. 2014.

[65] N. Nguyen-Trong, L. Hall, and C. Fumeaux, "Transmission-Line Model of Nonuniform Leaky-Wave Antennas," IEEE Trans. Antennas Propag., vol. 64, no. 3, pp. 883-893, 2016, doi: 10.1109/TAP.2016.2517669.

[66] J. Guo, Z. Li, J. Wang, M. Chen, and Z. Zhan. "Analysis and Design of Leaky-Wave Antenna with Low SLL Based on Half-Mode SIW Structure," Int. Journal Antennas Propag., Hindawi Publishing Corporation, Feb. 2015.

[67] X. Zou, C. M. Tong, H. He, and F. Geng, "Edge-radiating slot antenna based on half-mode substrate integrated waveguide," IET Microwaves, Antennas Propag., vol. 11, no. 8, pp. 1106-1112, 2017, doi: 10.1049/iet-map.2016.0884.

[68] Y. Cheng, X. Ding, W. Shao, and C. Liao, "A High-gain phased array with wide-angle scanning performance and low side-lobe levels," IEEE Access, vol. 7, pp. 31151-31158, 2019.

[69] N. Javanbakht, B. Syrett, and R. Amaya, "Leaky-Wave Antenna Based on Modified Aperture Half-Mode Substrate Integrated Waveguide," 2018 IEEE Antennas Propagation Society International Symposium and USNC/URSI 
National Radio Science Meeting, APSURSI 2018 - Proceedings, 2018, doi: 10.1109/APUSNCURSINRSM.2018.8608880.

[70] N. Javanbakht, B. Syrett, R. Amaya, and J. Shaker, "Side-lobe level reduction of half-mode substrate integrated waveguide leaky-wave antenna," IEEE Trans. Antennas Propag., vol. 68, Nov. 2020.

[71] A. Sarkar and S. Lim, "60 GHz Compact Larger Beam Scanning Range PCB Leaky-Wave Antenna Using HMSIW for Millimeter-Wave Applications," IEEE Trans. Antennas Propag., vol. 68, no. 8, pp. 5816-5826, Aug. 2020.

[72] N. Nguyen-Trong, T. Kaufmann, L. Hall, and C. Fumeaux, "Optimization of leaky-wave antennas based on non-uniform HMSIW," Proc. 2015 IEEE MTTS Int. Conf. Numer. Electromagn. Multiphysics Model. Optim. NEMO 2015, pp. 4-7, 2016, doi: 10.1109/NEMO.2015.7415002.

[73] X. Chen, Z. Li, H. Song, and J. Wang, "Generation of Radiation Null for the HMSIW Leaky-Wave Antenna," IEEE Antennas Wirel. Propag. Lett., vol. 16, no. c, pp. 2688-2691, 2017, doi: 10.1109/LAWP.2017.2741099.

[74] N. Nguyen-Trong, T. Kaufmann, and C. Fumeaux, "A wideband omnidirectional horizontally polarized traveling-wave antenna based on halfmode substrate integrated waveguide," IEEE Antennas Wirel. Propag. Lett., vol. 12, pp. 682-685, 2013, doi: 10.1109/LAWP.2013.2263492.

[75] N. Nguyen-Trong, T. Kaufmann, and C. Fumeaux, "A semi-analytical solution of a tapered half-mode substrate-integrated waveguide with application to rapid antenna optimization," IEEE Trans. Antennas Propag., vol. 62, no. 6, pp. 3189-3200, 2014, doi: 10.1109/TAP.2014.2314743. 

propagation properties of the half-mode substrate integrated waveguide," IEEE Trans. Microw. Theory Tech., vol. 57, no. 8, pp. 1996-2004, 2009, doi: 10.1109/TMTT.2009.2025429.

[77] A. Bhutani, B. Gottel, A. Lipp, and T. Zwick, "Packaging solution based on low-temperature cofired ceramic technology for frequencies beyond 100 GHz," IEEE Trans. Components, Packag. Manuf. Technol., vol. 9, no. 5, pp. $945-$ 954, 2019, doi: 10.1109/TCPMT.2018.2882062.

[78] U. Ullah et al., "Antenna in LTCC Technologies: A Review and the Current State of the Art," IEEE Antennas Propag. Mag., vol. 57, no. 2, pp. 241260, 2015, doi: 10.1109/MAP.2015.2414668.

[79] Z. Liu, P. Wang, and Z. Zeng, "Enhancement of the gain for microstrip antennas using negative permeability metamaterial on low temperature Co-fired ceramic (LTCC) Substrate," IEEE Antennas Wirel. Propag. Lett., vol. 12, pp. 429-432, 2013, doi: 10.1109/LAWP.2013.2254697.

[80] S. B. Yeap, Z. N. Chen, and X. Qing, "Gain-enhanced 60-GHz LTCC antenna array with open air cavities," IEEE Trans. Antennas Propag., vol. 59, no. 9, pp. 3470-3473, 2011, doi: 10.1109/TAP.2011.2161549. step-profiled corrugated horn antennas integrated in LTCC," IEEE Trans. Antennas Propag., $\quad$ vol. 62 , no. 11, pp. 5437-5444, 2014, doi: 10.1109/TAP.2014.2350520. 
waveguide and transitions for millimeter-wave applications," IEEE Trans. Microw. Theory Tech., vol. 67, no. 3, pp. 868-882, 2019, doi: 10.1109/TMTT.2019.2892743.

[83] F. Bauer and W. Menzel, “A 79-GHz planar antenna array using ceramicfilled cavity resonators in LTCC," IEEE Antennas Wirel. Propag. Lett., vol. 12, pp. 910-913, 2013, doi: 10.1109/LAWP.2013.2272914.

[84] J. Xu, Z. N. Chen, X. Qing, and W. Hong, "140-GHz planar broadband LTCC SIW slot antenna array," IEEE Trans. Antennas Propag., vol. 60, no. 6, pp. 3025-3028, 2012, doi: 10.1109/TAP.2012.2194673. W. Yang, Y. Yang, W. Che, C. Fan, and Q. Xue, "94-GHz Compact 2-D Multibeam LTCC Antenna Based on Multifolded SIW Beam-Forming Network," IEEE Trans. Antennas Propag., vol. 65, no. 8, pp. 4328-4333, 2017, doi: 10.1109/TAP.2017.2710229.

[86] N. Javanbakht, B. Syrett, R. E. Amaya, and J. Shaker, "Low Temperature Co-Fired Ceramic Half-Mode Substrate Integrated Waveguide Leaky-Wave Antenna," in Proceedings - ANTEM 2018: 2018 18th International Symposium on Antenna Technology and Applied Electromagnetics, 2018, vol. 2018-Augus, doi: 10.1109/ANTEM.2018.8572951.

[87] N. Javanbakht, B. Syrett, R. Amaya, and J. Shaker, "A compact cavitybased leaky-wave antenna in a low temperature co-fired ceramic process with improved performance," IEEE Access, vol. 9, Feb. 2021. B. Cao, H. Wang, Y. Huang, and J. Zheng, "High gain L-probe excited substrate integrated cavity antenna array with LTCC based gap waveguide 
feeding network for W-band application," IEEE Trans. Antennas Propag., vol. 63, no. 12, pp. 5465-5474, Dec. 2015.

$$
\text { F. Foglia Manzillo, et al., "A Multilayer LTCC solution for integrated 5G }
$$
access point antenna modules," IEEE Trans. Microw. Theo. Tech., vol. 64, no. 7, pp. 2272-2283, Jul. 2016.

[90] Nafe, F. Ghaffar, M. Farooqui, and A. Shamim, "A ferrite LTCC-based monolithic SIW phased antenna array," IEEE Trans. Antennas Propag., vol. 65, no. 1, pp. 196-205, Jan. 2017.

[91] Z. Miao, et al., "140 GHz High-gain LTCC integrated transmit-array antenna using a wideband SIW aperture coupling phase delay structure," IEEE Trans. Antennas Propag., vol. 66, no. 1, pp. 182-190, Jan. 2018.

[92] P. Li, Q. Xue, and Sh. Qu, “60 GHz Dual-polarized high gain planar aperture antenna array based on LTCC," IEEE Trans. Antennas Propag., vol. 68, no. 4, pp. 2883-2894, Apr. 2020.

P. Piasecki, Y. Yashchyshyn, A. Denisov, "Investigation of LTCC leakywave antenna in mm-wave band," $21^{\text {st }}$ Int. Conf. Microw. Radar Wireless Comm., Krakow, 2016.

S. Muhammad, A. Ali, Z. Ahmed, and M. Bin Ihsan, "Beam-steering in HMSIW LWA at fixed millimeter wave Frequency," 12 Int. Conf. High-capacity Optical Networks Emerg. Tech., pp. 91-92, 2015.

[95] M. Singh and B. Ghosh, "Periodic strip loaded reconfigurable half-mode substrate integrated waveguide-based leaky wave antennas," IEEE Electronics Letters, vol. 56, no. 13, pp. 646-648, Jun. 2020. 
[96] D. K. Karmokar, D. N. P. Thalakotuna, K. P. Esselle, L. Matekovits, and M. Heimlich, "Reconfigurable half-width microstrip leaky-wave antenna for fixed-frequency beam scanning," 2013 7th Eur. Conf. Antennas Propagation, EuCAP 2013, vol. 1, no. c, pp. 1314-1317, 2013. Steering of Microstrip Leaky-Wave Antennas Using Binary Switches," IEEE Trans. Antennas Propag., vol. 64, no. 6, pp. 2146-2154, 2016, doi: 10.1109/TAP.2016.2546949.

[98] M. K. Mohsen, M. S. M. Isa, A. A. M. Isa, M. K. Abdulhameed, and M. L. Attiah, "Achieving Fixed-Frequency Beam Scanning With a Microstrip LeakyWave Antenna Using Double-Gap Capacitor Technique," IEEE Antennas Wirel. Propag. Lett., vol. 18, no. 7, pp. 1502-1506, 2019, doi: 10.1109/LAWP.2019.2920940.

[99] A. Suntives and S. V Hum, "A Fixed-Frequency Beam-Steerable HalfMode Substrate Integrated Waveguide Leaky-Wave Antenna," IEEE Trans. Antennas Propag., vol. 60, no. 5, pp. 2540-2544, 2012.

[100] N. Javanbakht, B. Syrett, R. E. Amaya, and J. Shaker, "Miniaturized reconfigurable antenna based on half-mode substrate integrated waveguide," in SysCon 2019 - 13th Annual IEEE International Systems Conference, Proceedings, 2019, doi: 10.1109/SYSCON.2019.8836800.

[101] N. Javanbakht, B. Syrett, R. E. Amaya, and J. Shaker, "Electric beamsteering metamaterial leaky-wave antenna," in Proceedings of the 2019 21st International Conference on Electromagnetics in Advanced Applications, ICEAA 
2019, 2019, doi: 10.1109/ICEAA.2019.8879060.

[102] N. Javanbakht, B. Syrett, R. Amaya, and J. Shaker, "Electronic beamscanning leaky-wave antenna based on delta shape half-mode substrate integrated waveguide," Proc. 14th Eur. Conf. Antennas Propagation, EUCAP 2020, Copenhagen, 2020.

[103] N. Javanbakht, B. Syrett, R. Amaya, and J. Shaker, "Fixed frequency beam-scanning HMSIW-based leaky-wave antenna composed of circular slots in V-shape configuration," IEEE Access, vol. 9, Mar. 2021.

[104] A. Suntives and S. V. Hum, "An electronically tunable half-mode substrate integrated waveguide leaky-wave antenna," Proc. 5th Eur. Conf. Antennas Propagation, EUCAP 2011, pp. 3670-3674, 2011.

[105] D. K. Karmokar, D. N. P. Thalakotuna, K. P. Esselle, M. Heimlich, and L. Matekovits, "Fixed-frequency beam steering from a stub-loaded microstrip leakywave antenna," 2013 Int. Symp. Electromagn. Theory, EMTS 2013 - Proc., pp. 436-439, 2013.

[106] L. S. Wu et al., "A new type of periodically loaded half-mode substrate integrated waveguide and its applications," IEEE Trans. Microw. Theory Tech., vol. 58, no. 4, pp. 882-893, 2010, doi: 10.1109/TMTT.2010.2042832.

[107] A. Pourghorban Saghati, M. M. Mirsalehi, and M. H. Neshati, "A HMSIW circularly polarized leaky-wave antenna with backward, broadside, and forward radiation," IEEE Antennas Wirel. Propag. Lett., vol. 13, pp. 451-454, 2014, doi: 10.1109/LAWP.2014.2309557.

[108] Y. Geng, J. Wang, Y. Li, Z. Li, M. Chen, and Z. Zhang, "Radiation 
Pattern-Reconfigurable Leaky-Wave Antenna for Fixed-Frequency Beam Steering Based on Substrate-Integrated Waveguide," IEEE Antennas Wirel. Propag. Lett., vol. 18, no. 2, pp. 387-391, 2019, doi: 10.1109/LAWP.2019.2892057.

[109] T. Lou, X. X. Yang, H. Qiu, Q. Luo, and S. Gao, "Low-Cost Electrical Beam-Scanning Leaky-Wave Antenna Based on Bent Corrugated Substrate Integrated Waveguide," IEEE Antennas Wirel. Propag. Lett., vol. 18, no. 2, pp. 353-357, 2019, doi: 10.1109/LAWP.2019.2890995.

[110] K. Chen, Y. H. Zhang, S. Y. He, H. T. Chen, and G. Q. Zhu, "An electronically controlled leaky-wave antenna based on corrugated SIW structure with fixed-frequency beam scanning," IEEE Antennas Wirel. Propag. Lett., vol. 18, no. 3, pp. 551-555, 2019, doi: 10.1109/LAWP.2019.2896354.

[111] Y. Ji, L. Ge, J. Wang, Q. Chen, and W. Wu, "Simple Beam Scanning SIW Cavity-Backed Slot Antenna Using Postloaded Varactor," IEEE Antennas Wirel. Propag. Lett., vol. $18, \quad$ no. 12, pp. 2761-2765, 2019, doi: 10.1109/LAWP.2019.2951447.

[112] N. Javanbakht, B. Syrett, R. E. Amaya, and J. Shaker, "Electronically Steerable Quasi-Uniform Leaky-Wave Antenna Based on Substrate Integrated Waveguide," in 2019 IEEE Canadian Conference of Electrical and Computer Engineering, CCECE 2019, 2019, doi: 10.1109/CCECE.2019.8861758.

[113] N. Javanbakht, B. Syrett, R. E. Amaya, and J. Shaker, "Non-Uniform Steerable Leaky-Wave Antenna Based on Substrate Integrated Waveguide," in IEEE International Symposium on Phased Array Systems and Technology, 2019, 
vol. 2019-Octob, doi: 10.1109/PAST43306.2019.9020897.

[114] N. Javanbakht, B. Syrett, R. Amaya, and J. Shaker, "Electronic steerable slotted leaky-wave antenna," 2020 IEEE Antennas Propagation Society International Symposium USNC/URSI National Radio Science Meeting, APSURSI 2020, Montreal, 2020.

[115] N. Javanbakht, B. Syrett, R. Amaya, and J. Shaker, "Reconfigurable SIWbased leaky-wave antenna composed of longitudinal slots," IEEE Antennas. Wirel. Propag. Lett., submitted for publication.

[116] N. Javanbakht, B. Syrett, R. Amaya, and J. Shaker, "Miniaturized reconfigurable SIW-based leaky-wave antenna loaded with inclined slot," IEEE Int. Symp. Antennas Propag. USNC-URSI Radio Science Meeting, Singapore, submitted for publication, 2021.

[117] N. Javanbakht, B. Syrett, R. Amaya, and J. Shaker, "Reconfigurable antenna based on gap substrate integrated waveguide," 2020 IEEE Antennas Propagation Society International Symposium USNC/URSI National Radio Science Meeting, APSURSI 2020, Montreal, 2020. Leaky-Wave Antenna with Fixed-Frequency and Wide-Angle Beam Scanning," IEEE Trans. Antennas Propag., vol. 67, no. 6, pp. 3720-3732, 2019, doi: 10.1109/TAP.2019.2907636.

[119] L. Ge, Y. Li, J. Wang, and C. Y. D. Sim, "A low-profile reconfigurable cavity-backed slot antenna with frequency, polarization, and radiation pattern agility," IEEE Trans. Antennas Propag., vol. 65, no. 5, pp. 2182-2189, 2017, doi: 
10.1109/TAP.2017.2681432.

[120] N. Jess, B. Syrett, L. Roy, and R. E. Amaya, "Modeling substrate integrated waveguide structures using effective material properties," Proc. 2015 IEEE MTT-S Int. Conf. Numer. Electromagn. Multiphysics Model. Optim. NEMO 2015, pp. 6-8, 2016, doi: 10.1109/NEMO.2015.7415093.

[121] N. Jess, B. A. Syrett, and L. Roy, "The Field-Programmable Microwave Substrate," IEEE Trans. Microw. Theory Tech., vol. 64, no. 11, pp. 3469-3482, 2016, doi: 10.1109/TMTT.2016.2604315.

[122] P. Lotfi, S. Soltani, and R. D. Murch, "Printed Endfire Beam-Steerable Pixel Antenna," IEEE Trans. Antennas Propag., vol. 65, no. 8, pp. 3913-3923, 2017, doi: 10.1109/TAP.2017.2716399.

[123] D. Rodrigo and L. Jofre, "Frequency and radiation pattern reconfigurability of a multi-size pixel antenna," IEEE Trans. Antennas Propag., vol. 60, no. 5, pp. 2219-2225, 2012, doi: 10.1109/TAP.2012.2189739.

[124] S. Soltani, P. Lotfi, and R. Murch, "Design and optimization of multiport pixel antennas," IEEE Trans. Antennas Propag., vol. 66, no.4, pp. 2049-2054, Apr. 2018.

[125] L. N. Pringle, et al., "A reconfigurable aperture antenna based on switched links between electrically small metallic patches," IEEE Trans. Antennas Propag., vol. 52, no. 6, pp. 1434-1445, 2004, doi: 10.1109/TAP.2004.825648.

[126] Darvazehban, S. A. Rezaeieh, and A. M. Abbosh, "Programmable meta surface antenna for electromagnetic torso scanning," IEEE Access, vol. 8, pp. 166801-166812, Sep. 2020. 
[127] G. Oliveri, D. H. Werner, and A. Massa, "Reconfigurable electromagnetics through metamaterials-a review," Proc. IEEE, vol. 103, no. 7, pp. 1034-1056, 2015, doi: 10.1109/JPROC.2015.2394292.

[128] S. Lim, C. Caloz, and T. Itoh, "Electronically scanned composite right/left handed microstrip leaky-wave antenna," IEEE Microw. Wirel. Components Lett., vol. 14, no. 6, pp. 277-279, 2004., doi: 10.1109/LMWC.2004.828008.

[129] S. Lim, C. Caloz, and T. Itoh, "Metamaterial-based electronically controlled transmission-line structure as a novel leaky-wave antenna with tunable radiation angle and beamwidth," IEEE Trans. Microw. Theory Tech., vol. 53, no. 1, pp. 161-172, 2005, doi: 10.1109/TMTT.2004.839927.

[130] S. L. Chen, D. K. Karmokar, Z. Li, P. Y. Qin, R. W. Ziolkowski, and Y. Jay Guo, "Continuous Beam Scanning at a Fixed Frequency with a Composite Right-/Left-Handed Leaky-Wave Antenna Operating over a Wide Frequency Band," IEEE Trans. Antennas Propag., vol. 67, no. 12, pp. 7272-7284, 2019, doi: 10.1109/TAP.2019.2935088.

[131] J. H. Fu et al., "An Electrically Controlled CRLH-Inspired Circularly Polarized Leaky-Wave Antenna," IEEE Antennas Wirel. Propag. Lett., vol. 16, pp. 760-763, 2017, doi: 10.1109/LAWP.2016.2601960.

[132] R. Shaw and M. K. Mandal, "Broadside scanning fixed frequency LWA with simultaneous electronic control of beam angle and beamwidth," IEEE Trans. Antennas Propag., vol. 68, no. 5, pp. 3504-3514, May 2020.

[133] Md. Towfiq, et al., "A reconfigurable antenna with beam steering and beamwidth variability for wireless communications," IEEE Trans. Antennas 
Propag., vol. 66, no. 10, pp. 5052-5063, Jul. 2018.

A. A. Oliner, "POLYTECHNIC INSTITUTE OF BROOKLYN 1963 Short Course on 'Microwave Field and Network Techniques ' diagrams," 1963.

[135] Z. L. Ma, L. J. Jiang, S. Gupta, and W. E. I. Sha, "Dispersion characteristics analysis of one dimensional multiple periodic structures and their applications to antennas," IEEE Trans. Antennas Propag., vol. 63, no. 1, pp. 113121, 2015, doi: 10.1109/TAP.2014.2366785.

[136] J. Liu, D. R. Jackson, and Y. Long, "Modal analysis of dielectric-filled rectangular waveguide with transverse slots," IEEE Trans. Antennas Propag., vol. 59, no. 9, pp. 3194-3203, 2011, doi: 10.1109/TAP.2011.2161444.

[137] D. Comite et al., "Design of a Polarization-Diverse Planar Leaky-Wave Antenna for Broadside Radiation," IEEE Access, vol. 7, pp. 28672-28683, 2019, doi: 10.1109/ACCESS.2019.2901446.

[138] S. K. Podilchak, P. Baccarelli, P. Burghignoli, A. P. Freundorfer and Y. M. M. Antar, "Optimization of a Planar "Bulls-Eye" Leaky-Wave Antenna Fed by a Printed Surface-Wave Source," IEEE Antennas Wirele. Propag. Lett., vol. 12, pp. 665-669, 2013, doi: 10.1109/LAWP.2013.2262572.

[139] S. K. Podilchak, L. Matekovits, A. P. Freundorfer, Y. M. M. Antar, and M. Orefice, "Controlled Leaky-Wave Radiation From a Planar Configuration of Width-Modulated Microstrip Lines," IEEE Trans. Antennas Propag., vol. 61, no. 10, pp. 4957-4972, Oct. 2013, doi: 10.1109/TAP.2013.2274791.

[140] D. M. Pozar, Microwave Engineering, 3rd ed. New York, NY, USA: Wiley, 2005. 
[141] C.A. Balanis, Advanced Engineering Electromagnetics, 2nd ed., New York: Wiley, 2012.

[142] F. Mesa and D. R. Jackson, "The danger of high-frequency spurious effects on wide microstrip line," IEEE Trans. Microw. Theory Tech., vol. 50, no. 12, pp. 2679-2689, 2002, doi: 10.1109/TMTT.2002.805133.

[143] T. Zvolensky, D. Chicherin, A. V. Räisänen, and C. Simovski, "Leakywave antenna based on micro-electromechanical systems-loaded microstrip line," IET Microwaves, Antennas Propag., vol. 5, no. 3, pp. 357-363, 2011, doi: 10.1049/iet-map.2010.0168.

[144] E. Erdil, K. Topalli, M. Unlu, O. A. Civi, and T. Akin, "Frequency tunable microstrip patch antenna using RF MEMS technology," IEEE Trans. Antennas Propag., vol. 55, no. 4, pp. 1193-1196, 2007, doi: 10.1109/TAP.2007.893426.

[145] L. Ma, N. Soin, M. H. Mohd Daut and S. F. Wan Muhamad Hatta, "Comprehensive Study on RF-MEMS Switches Used for 5G Scenario," IEEE Access, vol. 7, pp. 107506-107522, 2019, doi: 10.1109/ACCESS.2019.2932800.

[146] T. Hand and S. Cummer, "Characterization of tunable metamaterial elements using MEMS switches," IEEE Antennas Wirel. Propag. Lett., vol. 6, pp. 401-404, 2007, doi: 10.1109/LAWP.2007.902807.

[147] MACOM Technology solutions Inc. MAVR-000120-14110 Datasheet (5th ed.) [online]. Available: https://www.macom.com/products/productdetail/MAVR-000120-14110P.

[148] MACOM Technology solutions Inc. MAVR-011020-14110 Datasheet (2nd ed.) [online]. Available: https://www.macom.com/products/product- 
detail/MAVR-011020-1411.

[149] C. Caloz and T. Itoh, Electromagnetic Metamaterials: Transmission Line Theory and Microwave Applications: The Engineering Approach. 2005.

[150] C. Caloz, "Metamaterial dispersion engineering concepts and applications," Proc. IEEE, vol. 99, no. 10, pp. 1711-1719, 2011, doi: 10.1109/JPROC.2011.2114631.

[151] M. R. M. Hashemi and T. Itoh, "Evolution of composite right/left-handed leaky-wave antennas," Proc. IEEE, vol. 99, no. 10, pp. 1746-1754, 2011, doi: 10.1109/JPROC.2011.2157797.

[152] S. Otto, A. Rennings, K. Solbach, and C. Caloz, "Transmission line modeling and asymptotic formulas for periodic leaky-wave antennas scanning through broadside," IEEE Trans. Antennas Propag., vol. 59, no. 10, pp. 36953709, 2011, doi: 10.1109/TAP.2011.2163781.

[153] S. Otto, A. Al-Bassam, A. Rennings, K. Solbach, and C. Caloz, "Radiation efficiency of longitudinally symmetric and asymmetric periodic leaky-wave antennas," IEEE Antennas Wirel. Propag. Lett., vol. 11, no. 2, pp. 612-615, 2012, doi: 10.1109/LAWP.2012.2202365.

[154] S. Otto, A. Al-Bassam, A. Rennings, K. Solbach, and C. Caloz, "Transversal asymmetry in periodic leaky-wave antennas for bloch impedance and radiation efficiency equalization through broadside," IEEE Trans. Antennas Propag., vol. 62, no. 10, pp. 5037-5054, 2014, doi: 10.1109/TAP.2014.2343621.

[155] D. K. Karmokar, Y. J. Guo, S. L. Chen, and T. S. Bird, "Composite Right/Left-Handed Leaky-Wave Antennas for Wide-Angle Beam Scanning with 
Flexibly Chosen Frequency Range," IEEE Trans. Antennas Propag., vol. 68, no. 1, pp. 100-110, 2020, doi: 10.1109/TAP.2019.2946750.

[156] Y. Dong and T. Itoh, "Composite right/left-handed substrate integrated waveguide and half mode substrate integrated waveguide leaky-wave structures," IEEE Trans. Antennas Propag., vol. 59, no. 3, pp. 767-775, 2011, doi: 10.1109/TAP.2010.2103025.

[157] M. M. Sabahi, A. A. Heidari, and M. Movahhedi, "A Compact CRLH Circularly Polarized Leaky-Wave Antenna Based on Substrate-Integrated Waveguide," IEEE Trans. Antennas Propag., vol. 66, no. 9, pp. 4407-4414, 2018, doi: 10.1109/TAP.2018.2851278.

[158] F. P. Casares-miranda, S. Member, C. Camacho-peñalosa, C. Caloz, and S. Member, "High-Gain Active Composite Right / Left-Handed Leaky-Wave Antenna," vol. 54, no. 8, pp. 2292-2300, 2006.

[159] S. S. Haghighi, A. A. Heidari, and M. Movahhedi, "A three-band substrate integrated waveguide leaky-wave antenna based on composite right/left-handed structure," IEEE Trans. Antennas Propag., vol. 63, no. 10, pp. 4578-4582, 2015, doi: 10.1109/TAP.2015.2456951.

[160] M. Alibakhshikenari, et. al., "High-Isolation Leaky-Wave Array Antenna Based on CRLH Metamaterial Implemented on SIW with $\pm 30^{\circ}$ Frequency BeamScanning Capability at Millimeter-Waves," Electronics, 2019, vol. 8, pp. 642657.

[161] -, "New Compact Printed Leaky-Wave Antenna with Beam Steering," Microw. Optical Tech. Lett., Vol. 58, no. 1, Jan. 2016, pp 215-217. 

-, "New CRLH-Based Planar Slotted Antennas with Helical Inductors for Wireless Communication Systems, RF-Circuits and Microwave Devices at UHFSHF Bands," Springer Jour. Wireless Personal Commu., February 2017, Vol. 92, no. 3, pp. 1029-1038.

[163] -, "Beam-Scanning Leaky-Wave Antenna Based on CRLH-Metamaterial for Millimeter-Wave Applications," IET Microw. Antennas Propag., vol. 13, no. 8, Jul. 2019, pp. $1129-1133$.

[164] -, "Extended Aperture Miniature Antenna Based on CRLH Metamaterials for Wireless Communication Systems Operating Over UHF to C-Band," Radio Science, Vol. 53, no. 2, Feb.2018, pp. 154-165.

[165] -, "Wideband Printed Monopole Antenna for Application in Wireless Communication Systems," IET Microw. Antennas Propag., Vol. 12, no. 7, Jun. 2018, pp. $1222-1230$. 